\title{
AERIAL PROFILING OF TERRAIN TO DEFINE STREAM-VALLEY GEOMETRY-- STUDY REPORT
}

U.S. GEOLOGICAL SURVEY

Open-File Report 76-672

Contractor:

The Charles Stark Draper Laboratory, Inc.

Cambridge, Massachusetts 02139

Contract No. 14-08-0001-14548

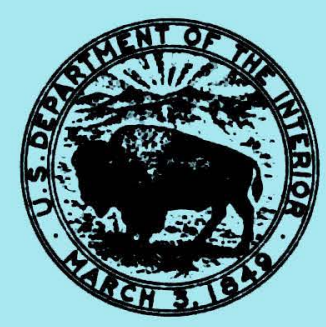




\section{AERIAL PROFILING OF TERRAIN TO DEFINE STREAM-VALLEY GEOMETRY-- STUDY REPORT}

By MUKUND DESAI, WILLIAM A. DROHAN, JOHN W. HURSH, GLENN MAMON, and DOUGLAS G. YOUMANS of The Charles Stark Draper Laboratory, Inc.

U.S. Geological Survey

Open-File Report 76-672

Contractor:

The Charles Stark Draper Laboratory, Inc.

Cambridge, Massachusetts 02139

Contract No. 14-08-0001-14548

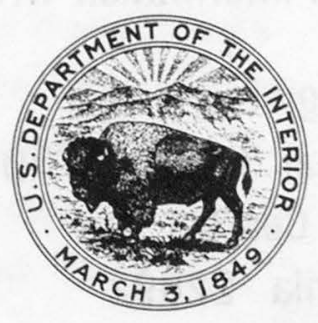




\section{U.S. DEPARTMENT OF THE INTERIOR \\ Thomas S. Kleppe, Secretary}

\section{GEOLOGICAL SURVEY}

V.E. McKelvey, Director

For additional information write to:

Chief Hydrologist

U.S. Geological Survey, WRD

409 National Center

Reston, Virginia 22092 
FOREWORD

Knowledge of locations and altitudes for points on land and on water in terms of earth-associated coordinate references became ever more important during the long climb of mankind from primeval beginnings to the sophisticated civilizations of today. It is certain that the requirements for such geographical information will in the future be increasingly stringent with trends toward higher accuracy over broader areas and greatly accelerated observations and transformations of data into high-quality maps. Results of this kind will depend upon methods and equipment capable of working rapidly and at low operating cost for extensive missions to realize good cost-effectiveness ratios.

This report is a study of possibilities in selfcontained aircraft-carried equipment to map rapidly those watershed features associated with flooding from selected streams. The specified performance requires precision of measurement to within \pm 0.5 foot in altitude and \pm 10 feet in geographical location. Using already-available groundcontrol points the total time to collect and display the desired data for an area of interest is to be a few tens of minutes. Geometrical information is to be generated by selfcontained mechanisms based on inertial principles, with an on-board reference member maintained in alignment with external earth-reference coordinates by means of servo-drives commanded by signals from angular deviation sensors. Other sensors carried by the reference member measure components of specific force -- the force per unit mass resulting from action by the earth's gravitational field and the reaction, in accord with Newton's Law, to the aircraft acceleration. The specific force components, suitably adjusted for 
gravitational effects by use of Schuler Tuning, serve as computer inputs for calculations of velocity and position changes. Relation to ground surveys is provided by an optical ranging device tracking ground-control points in the earth-coordinate system maintained by the on-board reference member, Altitude above points on the earth's surface is measured by applications of laser light interference patterns. Discussions, based on the information and reasoning presented in the body of this report, lead to the conclusion that systems incorporating inertial subsystems for navigation and laser subsystems for altitude will, with proper engineering, provide the mapping performance necessary to meet the requirements specified in the original agreement for study.

The operation of self-contained inertial systems, giving information on location, orientation, linear velocity, angular velocity, and the associated accelerations, provides functions for determining the components of geometrical quantities in the same way that operation of clocks, chronometers, watches, and oscillators serves to supply measures for time as a physical quantity. In all such situations the devices involved sense and produce signals representing changes in the physical quantity of interest. These signals arise because the devices exist within a universe in which certain laws of physics are true and where particular component materials exist, not because the devices measure any continuous contacts with outside space. Time keepers require setting if their indications are to be in terms of a particular time standard. Because outputs from indicators for geometrical quantities are not generally useful unless they are related to known coordinate-reference systems, they must be given initial alignment just as settings to time standards are normal practice for clocks, 
chronometers, watches, and oscillators. Reasonably accurate self-contained devices for indicating time have been in use for some seven hundred years. Technological progress during the centuries has reduced massive iron gear and pendulum mechanisms to small tuning forks and quartz crystal oscillator wrist watches that have improved performance by many orders of magnitude.

The application of self-contained equipment to geographical indications for the earth sciences may well be a very significant stage in the advancement of inertial navigation technology, Goals of a few feet in map-position uncertainties are in contrast to the 600 feet now accepted by users of commercially produced airline Inertial Navigation systems, In practical operations commercial systems currently build up location inaccuracies of 300 to 6000 feet for each elapsed hour of flight. Comparable performance for state-of-the-art systems should result in buildup of location inaccuracies more like 15 feet for each hour of flight.

The foregoing numbers show that the equipment proposed for addressing problems in the earth sciences has performance some two orders of magnitude better than the results now generally provided by commercial Inertial Navigation systems. There are strong reasons for assuming that potential progress is not limited to performance of the quality that would be provided by the proposed system. It may very well be that this report is only the start of a long and important path of advancement in all the activities of civilization for which indications of geometrical quantities are essential inputs. 


\section{ABSTRACT}

A six-month engineering analysis was performed by The Charles Stark Draper Laboratory, Inc., at the request of the U. S. Geological Survey, to investigate the suitability of an airborne instrument package based on inertial techniques to serve as the datum for a laser altimeter in a system for aerial profiling of terrain to determine selected features of stream-valley geometry to an accuracy of $\pm 0.5 \mathrm{ft}$. in the vertical coordinate and $\pm 10 \mathrm{ft}$, in the horizontal coordinates. Feasible system configuration features a high performance inertial platform incorporating an integral laser tracker, pointing and ranging on retroreflectors on the ground, in order to provide the frequent updates needed to meet the accuracy requirements. In all environments except those of severe gravity gradients the nominal two- by twentymile survey area can be covered using three ground-surveyed retroreflectors, interspersed with several unlocated retroreflectors that are surveyed in by the airborne system along a longitudinal path within the river valley when the aircraft arrives over the site. Subsequent transverse profiling runs (traverses that may be spaced as close as one-quarter mile apart) are flown using, in turn, all retroreflectors as updating position references. Pointing and range information from the tracker are optimally combined with the on-board inertial measurements and available gravity data to provide 
position information and serve as the height datum for a terrain-clearance measuring laser altimeter. Data-logging means and operator display, as well as steering commands to the aircraft autopilot, are provided. The system configuration is capable of operating in single- or twin-engine aircraft including helicopters. It is recommended that work proceed into the design phase. 
1. The accuracy and operational requirements for aerial profiling of terrain to reveal selected features of stream-valley geometry can be met by a system configuration featuring inertial and electroöptical instrumentation combined with statistical filterıng and smoothing techniques in software.

2. The most difficult requirement is vertical positionsystem indication to $\pm 6^{\prime \prime}$. The significant error sources in the inertial subsystem are vertical accelerometer bias* uncertainty and gravity anomalies. This subsystem requires a state-of-the-art integrating accelerometer with position updating and position-derived velocity updating approximately every 3 minutes. The vertical position measurements for updating must be accurate to a few inches.

3. Terrain clearance measurements to the required accuracy and resolution are best obtained by an amplitude modulated continuous wave laser in the altimeter as opposed to a pulse modulated laser. Height measurements are accomplished by heterodyning and performing an accurate phase measurement. Smoothing and averaging of range data can best be accomplished by a local microprocessor before the data are sent to the main computer.

4. The inertial system will provide short-term accuracy. Repeated measurement of the range and angular position of retroreflectors will provide long-term accuracy.

5. Updating the inertial system with the laser altimeter is impractical because the necessary, frequent updates would severely limit the efficiency of the profiling and greatly extend the length of the flights. A separate

Difference between the measured output and the expected output at zero input. 
laser tracker operating in conjunction with retroreflectors on the ground eliminates this difficulty. By ranging and measuring the tracking angles while a sequence of retroreflectors is viewed, position updating and position-derived velocity updating in all three coordinates can be accomplished.

6. The inertial system has a unique capability to precisely locate unsurveyed retroreflectors with respect to surveyed retroreflectors during initial flights lengthwise of the river valley. These can be used subsequently, along with the surveyed retroreflectors, for updating the system during the transverse profiling runs. With this procedure, only three ground-surveyed retroreflectors are required, but these must not be in a straight line.

7. The simplest, most accurate mechanization of the airborne survey package mounts the laser tracker for updating on the stable member of the inertial measurement unit (IMU). In this configuration the tracker is stabilized in the same coordinate system as the accelerometers by appropriate servo drives.

8. Commercially available gyros and accelerometers (except for the vertical accelerometer, see paragraph 2 above) will meet the performance requirements for streamvalley profiling. Use of state-of-the-art (extremely low drift) gyros will allow isolation of gravity anomaly effects and enhance the growth potential of the system for other topological and geological survey applications.

9. With proper optical design, the laser altimeter can be designed so that the required laser power is proportional to the square of the range rather than the fourth power. A helium neon laser of slightly more than 20 milliwatts power will be adequate for the laser altimeter. A 10 milliwatt powered gallium arsenide laser or helium neon 
laser will be adequate for the tracker. Effects of air density variations on range measurements are small, but first-order corrections should be made to the index of refraction based on pressure and temperature measurements. 
1. Proceed with the design phase of the recommended configuration for the airborne survey system consisting of:

- Inertial measurement unit-tracker assembly and associated electronics

- Gimbaled laser altimeter and associated electronics and either video or film camera

- Digital computer and magnetic tape recorder

- Control and display unit

2. Carry out the design work to, insofar as possible, meet the accuracy and operating requirements of the variety of applications to field-survey problems specified by the Geological Survey including stream-valley profiling.

3. During the design phase, plan for efficient transition to the fabrication, laboratory-test, and flighttest phases, and finally field trials at one or more streamvalley sites, providing the USGS with a capable and versatile airborne data-gathering system.

4. Provide sufficient documentation to fabricate the prototype system and serve as the basis for release to larger-scale manufacturing when required. 
TABLE OF CONTENTS

Section

Page

1

INTRODUCTION

1.1 Stream-Valley Profiling 1

1.2 The Ideal Profiling system 2

1.3 Objective 3

1.4 Approach 3

2

STUDY OVERVIEW

2.1 Ground Rules For Study 7

2.2 General and Specific System Requirements 9

2.3 Major Trade-offs Involved in Meeting Requirements

2.3.1 Airborne-Position Determination 11

2.3.2 Laser Altimeter 19

2.3.3 Laser Tracker for Updating Separate from Laser Altimeter 21

2.3.4 Aircraft Flight Strategies 25

2.4 Choice of System Elements and optimum System Configuration

2.4.1 Overall System

2.4.2 Criteria for selection of

Inertial Components

2.4.2.1 Analysis of Inertial System Error Growth

2.4.2.2 Gravity Anomaly Produced Errors

2.4.2.3 Selection of Inertial Components 


\section{Table of Contents (Cont'd)}

Section

Page

2.4.3 Criteria for Selection of Electroöptical Components

2.4.3.1 Laser Safety

Considerations

60

2.4.4 Alignment and Calibration 61

2.4.5 Subsystem Interfaces 62

2.5 Alternate System Applications 66

2.5.1 Topographical Surveys 67

2.5.2 Geological Surveys 68

3.1 System Organization 69

3.2 Cost Considerations 71

3.3 Power Considerations 72

3.4 Size and Weight Considerations 72

4 RESULTS IN MAJOR STUDY AREAS

4.1 Analysis, Estimation and Simulation
of Errors in Aerial Surveying

4.1.1 Introduction 75

4.1.2 Position Errors in Aerial Surveying 76

4.1.2.1 Introduction 76

4.1.2.2 Error Sources in Determining Aircraft Position 78

4.1.2.3 Discussion of Various Error Sources 83

(a) Inertial System Errors 83

(b) Effect of Instrument and Gravity Model

Errors 


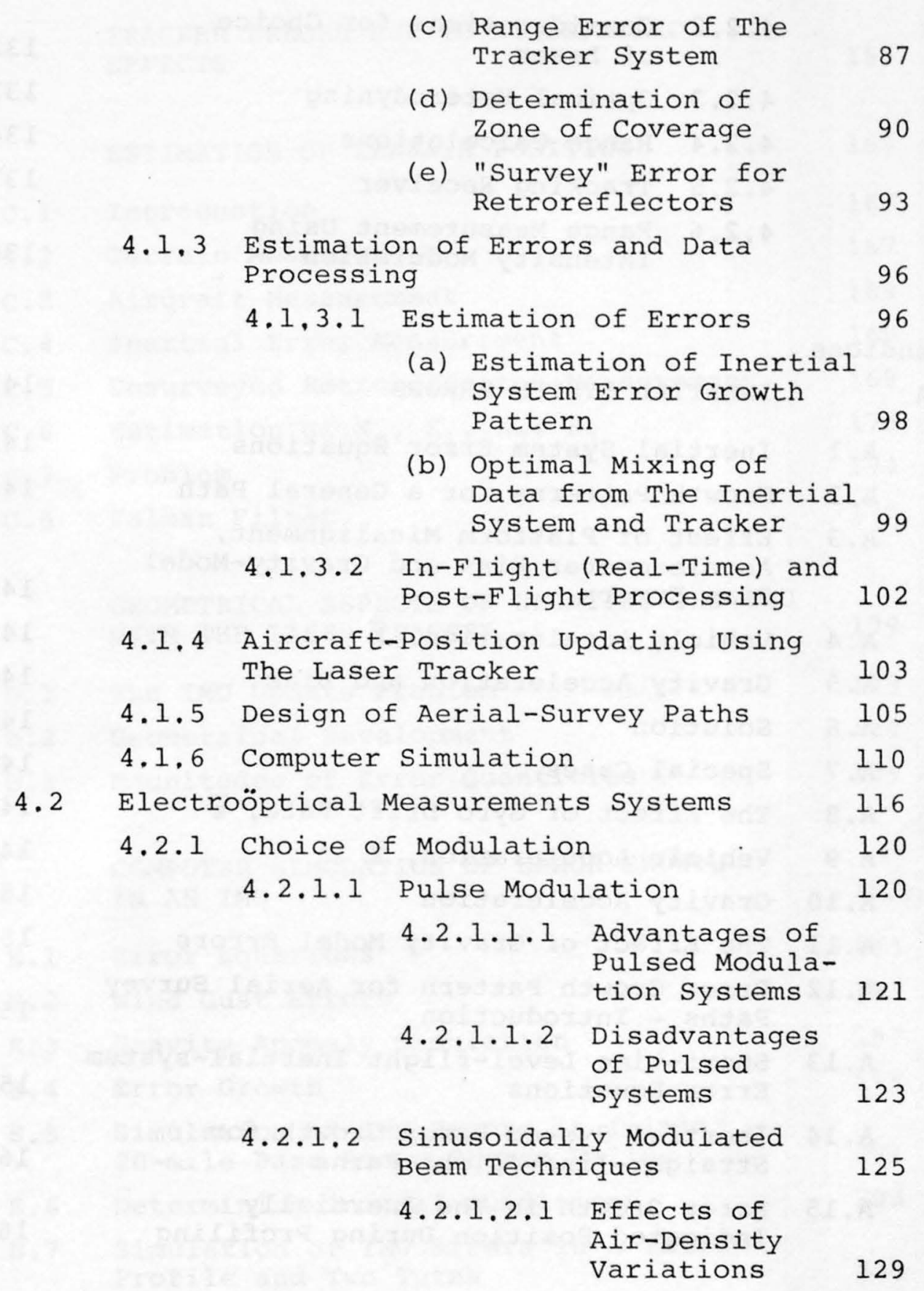




\section{Table of Contents (Cont'd)}

Section

Page

4.2.2 Considerations for Choice
of Laser

4.2.3 Optical Heterodyning

4.2.4 Range Calculations

4.2.5 Tracking Receiver

4.2.6 Range Measurement Using Intensity Modulation

Appendices

A

INERTIAL SYSTEM ERRORS

143

A.1 Inertial System Error Equations

143

A.2 Growth Patterns for a General Path

144

A. 3 Effect of Platform Misalignment, Accelerometer Bias and Gravity-Model Bias Errors

A. 4 Vehicle Acceleration, $\ddot{\bar{x}}$

145

A. 5 Gravity Acceleration and Bias

146

A. 6 Solution

146

A. 7 Special Cases

147

A. 8 The Effect of Gyro Drift Rate, $\bar{\varepsilon}$

149

A. 9 Vehicle Acceleration, $\overline{\mathrm{X}}$

149

A. 10 Gravity Acceleration

153

A.11 The Effect of Gravity Model Errors

154

A.12 Error Growth Pattern for Aerial Survey Paths - Introduction

A.13 Short-time Level-flight Inertial-system Error Equations

A.14 Inertial System Error Growth for Straight-line Flight Paths

A.15 Error Growth in The Inertially Indicated Position During Profiling 


\section{Table of Contents (Cont'd)}

Appendices

Page

B

TRACKER ERRORS DUE TO ATMOSPHERIC EFFECTS

C ESTIMATION OF TERRAIN POSITION

C.1 Introduction

C. 2 Terrain Measurement

C. 3 Aircraft Measurement

C. 4 Inertial Error Measurement

C. 5 Unsurveyed Retroreflector Measurement

C. 6 Estimation of $\mathrm{K}_{0}, \mathrm{~K}_{1}, \mathrm{~K}_{2}, \mathrm{~K}_{3}$

C. 7 Problem

C. 8 Kalman Filter

D GEOMETRICAL ASPECTS OF UPDATING THE IMU WITH THE LASER TRACKER

D.1 The IMU Update Problem

D. 2 Geometrical Development

D. 3 Magnitudes of Error Quantities

E COMPUTER SIMULATION OF ERROR GROWTH IN AN IMU

E.1 Error Equations

E.2 Wind Gust Errors

E. 3 Gravity Anomaly Simulation

E. 4 Error Growth

E.5 Simulation of IMU Errors in Initial 20-mile Pass Along Stream Valley

E. 6 Determining Initial Conditions

E.7 Simulation of IMU Errors in A Valley Profile and Two Turns 
Appendices

Page

F

SIGNAL-TO-NOISE CONSIDERATIONS FOR A PULSED LASER RANGEFINDER

G

BACKGROUND NOISE CALCULATIONS

$\mathrm{H}$

SIGNAL-TO-NOISE RATIO CONSIDERATIONS AND RECEIVED POWER

I

POWER RECEIVED BY ALTIMETER AND TRACKER

215

I.1 General

215

I.2 Laser Altimeter

215

I. 3 Laser Tracker

216

$\mathrm{J}$

TRANSMITTER POWER DETERMINATION

J.1 Laser Altimeter

221

J.2 Laser Tracker

K

MODULATION FREQUENCY REQUIRED FOR

DIFFERENT VALUES OF RANGE RESOLUTION 
2.3-1 Frequency characteristics of IMU and position fix noise

2.3-2 Return signals from various types of terrain

2.3-3 Maury River between Lexington and Buena Vista, Va., showing cross sections needed to define stream-valley geometry

2.4-1 Airborne profiling system

2.4-2 Mechanical schematic of IMU and laser-tracker gimbaling

2.4-3 Conceptual disposition of instrument package in aircraft

2.4-4 Relative contributions of gyros and accelerometers to horizontal errors

2.4-5 Vertical channel error due to vertical accelerometer bias

2.4-6 Vertical channel error induced by horizontal acceleration

2.4-7 Relation of gravity gradient to gyro drift

2.4-8 Gyro drift versus horizontal gravity gradient at three velocities of aircraft 51

2.4-9 Separation of gravity gradient and instrument errors

2.4-10 Vertical error sources

2.4-11 Major interfaces with IMU

2. $4-12$

Major interfaces with laser tracker 
Fig. No.

4.1-1 Geometry of aerial profiling

$4 \cdot 1-2$ Geometry of Fig, 4.1-1 with addition of retroreflector survey error, $\delta \overline{\mathrm{x}}_{r r^{\prime}}$ and error in retroreflector position, $\delta \bar{x}_{r}$

4.1-3 Reference frames

$4 \cdot 1-4$ Effect of range and angle measurement errors

$4 \cdot 1-5$

Position error, $\delta \bar{x}_{r}$, of aircraft at $A$ composed of range êrror, $\delta r$, and orientation error, $r d \theta$

$4 \cdot 1-6$

Determination of position of unsurveyed retroreflectors

$4,1-7$

Filtering and smoothing of position measurements

$4,1-8$

Straight flight along river valley

$4,1-9$ Flight to measure transverse cross sections of river valley

4.1-10 Longitudinal pass error growth

4.1-11

Growth of errors with time in profile mode - worst case profile with gravity anomaly

4.2-1 Last pulse selection

$4,2-2$

Resolution of pulsed lasers

122

$4,2-3$

Optical tracker

128

$4.2-4$

Four-quadrant tracking receiver

$4.2-5$

Image intensifier tracking receiver

140

$4,2-6$

Ranging Receiver

141

$\mathrm{A}-1$

Flight-path profile

$\mathrm{A}-2$

Flight-path diagram

152

A-3

Typical case of gravity anomalies

and deflections - Denver

156 
Fig. No.

$$
\text { Title }
$$

Page

A-4 Worst case of gravity anomalies and deflections - Des Moines

A-5 Horizontal position error growth pattern during profiling

A-6 Vertical position error growth pattern during profiling

164

C-1 Measurement geometry during aerial surveying

D-1 Geometry of aircraft and retroreflector used in analysis of method of updating the IMU with laser tracker

Inertial measurement system

$\mathrm{E}-2$

Equations for error growth in an IMU

$\mathrm{E}-3$

Gravity anomaly estimation technique

199

$\mathrm{E}-4$

Longitudinal pass error growth with

filtering and smoothing

202

$I-1$

Diagram of optical tracker

217 


\section{LIST OF TABLES}

Table No.

Page

4.1-1 Significant Sources of Error

4.1-2 Longitudinal Pass Errors - Vertical Channel

4.1-3 Longitudinal Pass Errors - Horizontal Channels

4. 1-4

Profile Mode Errors - Vertical Channel

4. $1-5$

Profile Mode Errors - Horizontal

Channels 
SECTION 1

INTRODUCTION

\subsection{Stream-Valley Profiling}

The U. S. Geological Survey maintains a network of nearly 10,000 gaging stations on streams and rivers throughout the United States, At each of these gaging stations the relationship between stream stage and discharge has been established quite accurately through careful measurement and calculations involving the profile of the riverbed and velocities measured over the cross section of the stream. Stream-stage data and corresponding discharge measurements for a good many years are available for the majority of the gaging stations. With stream-valley terrain-profile data from selected stream-crossing sites, upstream and downstream of the gaging station, hydrologists can fit flood stage discharge values into the valley geometry and thereby determine the stream profile corresponding to each flood magnitude. Flood limit contours can then be drawn for the stream valley. The needed profile data are now being obtained by means of photogrammetry and conventional surveying techniques. Obtaining the data to the required accuracy is quite timeconsuming and therefore expensive.

In view of the continuing urgent need for floodprobability contour maps, the U. S. Geological Survey has, over the past two years, become increasingly concerned with investigating the feasibility of alternative means for 
obtaining the requisite terrain-profile data much more rapidly than is possible at present, with no sacrifice in accuracy standarảs and, if possible, at reduced cost.

\subsection{The Ideal Profiling System}

Experience with aerial-surveying techniques has established the advantages of speed, minimum interference with the terrain under survey and ease of deployment from one site to another. The conclusion follows that the ideal system for stream-valley profiling would be carried by a relatively small, maneuverable aircraft and incorporate a surveyinginstrument package unaffected by aircraft motion capable of continuously providing an error-free, 3-coordinate position datum and vertical stabilization for a self-contained terrain-clearance measuring device and parallel automatic camera or other imaging device for recording the character of the stream valley being profiled. A data-logging system, preferably compatible with large-scale computers, a master clock and operator display for in-flight monitoring of overall performance, would complete the system.

In a typical mission, the cognizant hydrologist would select the locations of the desired stream-valley profiles, designating them on an appropriately scaled map for use by the survey crew consisting of the pilot and system operator, who might also serve as copilot. The system would be set in operation at an airfield convenient to the survey site, then flown to the location of the first stream-valley profiling traverse. After in-flight calibration of the instrument system, by sighting successively on three pre-surveyed ground-control points in or near the local survey areas, the survey would then proceed according to plan, crisscrossing the valley while logging data for the desired profiles with the operator observing the display to be sure 
that data of the requisite quality were being gathered.

It is clear that such an ideal system would be capable of gathering the required stream-valley profile data for an area many miles long in a very few hours. Very little advance-site preparation or other on-ground activities would be required. All necessary equipment would be carried aboard the aircraft, and could be easily deployed from one survey site to another.

While ideal systems are seldom capable of attainment, the attractiveness of these concepts for a self-contained airborne-surveying package led to serious consideration by the U. S. Geological Survey of systems mechanizations that might be expected to approach the ideal, and to the establishment of performance and accuracy requirements for such a system. These investigations have concluded that the airborne-surveying package to be investigated should include a laser-based, ranging altimeter to measure terrain clearance, and inertial equipment, consisting of an inertial measurement unit (IMU) with associated computer, to provide the position datum and pointing stabilization,

\subsection{Objective}

The study documented in this report was undertaken by The Charles Stark Draper Laboratory, Inc, under sponsorship of the U. S. Geological survey to investigate the feasibility of developing an airborne-instrument package capable of providing stream-valley profiling data to current accuracy standards at the same time accomplishing substantial savings in field work, time and costs.

\subsection{Approach}

The study began on June 24, 1974, culminating in a briefing on the significant findings held on February 3, 1975, at the USGS National Center and is complete with the 
publication of this report.

The work consisted of engineering analyses, preliminary system design and computer simulation, organized to establish, insofar as possible within the time and funding resources available, specifics in the following areas:

- Performance parameters of system components All significant error sources and inaccuracies in system elements and components were examined, and their effects on the performance of the subsystem of which they were a part were derived, together with the effect of the summation of these errors on overall system accuracy. In addition to the inertial system components the analysis also included gravity anomalies, optical components, electronics and propagation effects associated with the laser-ranging devices, and aircraft-environmental factors.

- Operating procedures and criteria - Because the sole purpose of the mission is to gather profiling data, an important area of investigation was the optimization of operating procedures to favor data accuracy (minimize error growth).

- Ground-truth requirements - Even the ideal system would require three surveyed ground-control points to tie the data gathered by the airborne instrument package to the ground. Because the proposed instrument system was found early in the course of the work to require tie-in to ground truth at about 3-minute intervals, for the purpose of updating position, provision for, and the spacing of, suitable ground reference marks assumed considerable importance in the study.

- System configuration - Here the attention was 
focussed on the fundamental trade-off between high performance and attendant complexity in the airborne portions of the system versus dependence on extensive site preparation and dependence on ground-deployed reference marks or equipment. Considerable emphasis was placed on retaining the operational flexibility and minimum site preparation requirements of the ideal system outlined above.

- Cost factors - Use of components in subsystems already in government inventory, as well as commercially available equipment, was carefully considered in order to minimize development and production costs of the airborne-survey package as well as the remaining portions of the overall system.

Conclusions and recommendations arising from the study are concisely enumerated in the first pages of this report. Succeeding sections of the report include a study overview wherein ground rules for the study, system requirements and major trade-offs, as well as choice of elements in optimum system organization are presented, followed by a section summarizing the major features of the recommended system configuration. The final section of the report presents results in the major study areas. Complete and detailed descriptions and mathematical developments in support of the results in the major study areas are contained in the Appendices. 
SECTION 2

STUDY OVERVIEW

\subsection{Ground Rules For Study}

In order to provide a focus for the investigation and appropriately bound the many and various possible system configurations that might be considered, the following ground rules were established by the Geological Survey. Each will be discussed in turn as it relates to the course of the study.

- Three-Axis IMU, Laser-Altimeter Principal Components of Airborne Instrument Package

Since no extreme maneuvering of the aircraft is anticipated, no redundant drive axis will be required in the IMU. A 3-axis design with roll and pitch capability up to $60^{\circ}$ appears to be adequate. Early in the study, vertical position indication was found to be the pacing requirement in the design of the inertial system. To provide the most favorable environment for the vertical accelerometer, its sensitive axis is mounted parallel to the inner axis of the IMU which is, in turn, aligned with the direction of vertical. This alignment is maintained by the system during flight within the position-indicating accuracy of the system. This arrangement is known as a vertical tracking system mechanization. The outer axis of the IMU is parallel to the roll axis of the aircraft, the middle 
axis -- parallel to the aircraft pitch axis in level of flight - being held perpendicular to the outer two through appropriate gimbaling .

At the outset of the study it was believed feasible for the laser altimeter to provide both terrain-profiling data and position-update data. Early in the study it became apparent that, due to the rapid buildup of errors in the vertical channel, a position and velocity update would be needed every few minutes, Time-sequencing the dual utilization appeared to be a serious problem. This, together with the fact that the return signal from a retroreflector, such as might be tracked to provide ground truth, would be several orders of magnitude higher than the return from the terrain led to the suggestion that the dual functions might best be performed by separate instruments. From that point forward in the study separate instruments were considered; a laser tracker working in close association with the IMU and a laser altimeter directed downward along the vertical to provide groundclearance data.

- Function in Small Single- or Twin-Engine Aircraft at Altitudes of 2000 Feet or Less

The aircraft environment has very little effect on inertial system performance, provided the design makes proper allowance for the expected vibration and temperature ranges. Optical glass windows in the bottom of the fuselage would be needed for the tracker and altimeter. Also, an auxiliary power unit would probably be required in order that the system could be set in operation and calibration and alignment procedures carried out without the necessity of running the aircraft engine. 
With USGS concurrence, the operating altitude was increased to 3000 feet to provide for tracking the retroreflector ground-truth mark from greater distances, thereby extending the range of usefulness of the tracker for updating the inertial equipment.

- Gather Data at Arbitrarily Spaced (As Close as $\frac{1}{4}$ Mile) Cross Sections in Generalized Operating Area 2 Miles or Less in Width and 20 Miles or Less in Length

over $70 \%$ of the stream valleys to be surveyed are within this generalized operating area. Strategies for flying the required stream-valley traverses, number and location of update marks and over-all mission length all follow from the definition of the generalized operating area.

\section{2,2 General and Specific System Requirements}

The over-all performance requirements are the most important element in determining the characteristics of the system design. Specific requirements are listed below, together with their respective implications in the system design.

- Continuously provide 3-coordinate position information such that the accuracy of the topographic data shall be, $90 \%$ of the time, within $\pm 0.5 \mathrm{ft}$. in the vertical coordinate and $\pm 10 \mathrm{ft}$. in the horizontal coordinates.

The inertial system is a low-noise datum at relatively high frequencies, capable of accurately following aircraft motions through maneuvers or turbulent air, Sufficient resolution for measurement to the desired accuracy is easily provided for. On the other hand, inaccuracies in the accelerometers, gravity anomalies, and slow drift in the gyros cause accumulating errors in the low frequency portion of 
the spectrum, It was recognized at the outset that some type of updating from ground truth would be needed to provide the necessary accuracy relative to the stream valley. The fact that the accuracy requirement in the vertical coordinate is $1 \frac{1}{2}$ orders of magnitude higher than in the horizontal coordinates makes updating the vertical channel the pacing requirement. Early in the study it was found that these updates would be needed every few minutes, This had a profound effect on the course of the study.

- Tie Airborne Position Information Into Available Ground-based Control Points in Local Survey Areas This requirement is automatically met by the means which must be provided for frequent and accurate update of the airborne-position datum.

- Provide for Self-alignment and Self-calibration of The Instrument Package

Self-alignment and self-calibration would be performed in the aircraft automatically under control of the system digital computer using well-known techniques proven over the years in inertial-system applications.

- Provide Vertical Pointing Stabilization for The Laser Altimeter and A Small Camera

Vertical stabilization to an accuracy of about 0.1 degree is required for the laser altimeter and camera. These instruments would be mounted on a twoaxis servo-driven platform, the outer axis being parallel to the roll axis of the aircraft. The stabilization signals could then be provided directly by resolvers on the outer (roll) and missile axes of the IMU. Analog nservo loops would be used, and no particular problems are anticipated. 
2.3 Major Trade-offs Involved in Meeting Requirements

An ideal system for aerial profiling of terrain would consist of a perfect inertial-navigation system and a laser altimeter, The aircraft would leave the airport, fly over the river valley being surveyed, and simultaneously record the aircraft position and distance to the ground. In the event there would be no error in the aircraft position, terrain altitude would be determined simply by subtracting terrain range from aircraft position. Post-flight data processing would be extremely simple.

In the real situation, however, there are inertialnavigation system errors due to small biases in the inertial components and due to inaccuracies in knowing the gravitation field at each position along the flight path. Consequently, periodic calibration of the aircraft position is necessary. For high-accuracy position updates an optical-ranging device, such as the laser tracker, is required to determine aircraft position. The higher the quality of the inertial-navigation system, the fewer updates required up to the point where gravity anomaly-produced errors become dominant. The major trade-offs involved in meeting the USGS error requirements for aerial profiling of terrain are the subjects of this section.

\subsubsection{Airborne-Position Determination}

Given the requirement of obtaining topographic data from an aircraft to the accuracies stated in section 2.2, the system design immediately separates itself into two areas:

1. a means for obtaining the instantaneous vertical distance between the terrain and the aircraft

2. a means for obtaining the instantaneous location of the aircraft in a known coordinate frame. The combined 
system will then yield the height of the terrain, in terms of known map coordinates, above a known datum.

Both of the above problems must be attacked, with consistent emphasis to assure successful system design. The first is solved by a laser altimeter, and is described in detail in sections 2.3 .2 and 4.2. The second is the airborne navigation and pointing problem.

Establishment of the Airborne Datum involves two aspects :

a) a navigation requirement

b) a pointing requirement.

These two aspects are, in general, quite distinct, in the sense that one can navigate without providing a pointing datum. For instance, radio-position fixing, such as Loran, will locate the (horizontal) position of the aircraft in a known coordinate frame, but provides no instrument-pointing datum.

Pointing datums (i.e., geometric coordinate frames) are established by means of measuring or detecting the presence of known fixed vectors, such as gravity and the earth's rotation vector, and "remembering" these directions with gyroscopic instruments. The vertical attitude gyro (artificial horizon) in an aircraft is a good example of a pointing datum.

Inertial navigators are unique in that they combine the solution to the pointing-datum requirement and the navigation requirement in a single mechanism, an assemblage of gyros and accelerometers. The pointing datum is, in fact, an $x, y, z$ coordinate frame fixed to the inner, or stable, member of the IMU. This frame is called the "indicated" frame or "platform" frame, or "p" frame. We will henceforth call it the "p" frame. It can be initially established by a two-step ground-alignment process: 
1. levelling - establishment of the $x_{p}-y_{p}$ plane as normal to the gravity vector

2. gyrocompassing - establishment of the $y_{p}$ axis as normal to the earth's rotation vector.

The "p" frame is maintained in flight by "torquing" signals proportional to the change in latitude and longitude angles moved over the earth.

Navigation is performed by simply doubly integrating the outputs of a triad of accelerometers. However, accelerometers are scalar-measuring devices, and the requirement is to measure vector displacement. The requirement is therefore met by fixing the sensitive axes of the accelerometers with respect to the three coordinate axes of the "p" frame. The measurements then become vector measurements in a known coordinate frame. The displacements thus derived are used to compute the "tilt" signals to be applied to the "p" frame to keep the $x_{p}-y_{p}$ plane normal to local gravity. This "feedback" is what gives rise to the Schuler oscillation in the horizontal channels, and exponential error growth in the vertical channel.

Another distinctive feature of inertial navigators, as compared to direct position-fixing, is that the directly measured quantity is acceleration; velocity and position are obtained by integration, a noise-smoothing process. Thus the measurement-instrument noise is greatly decreased by the integration process. The result is that the high-frequency resolution of inertial navigators is unexcelled. In contrast, if one wishes to obtain continuous velocity information from, for instance, a set of laser-ranging devices, the measurementinstrument noise is greatly amplified by the required differentiation.

Since the airborne-datum requirement for the laser altimeter requires both high-frequency position information 
(i.e., high resolution) and a pointing datum, the use of an IMU is immediately and strongly indicated.

The system-accuracy requirements, as given in Sec. 2.2, are extremely high. In addition, the datum resolution, that is, the time and/or distance between independent position measurements, is high. This combination of high accuracy and high resolution is best met, possibly only met, by inertial techniques.

The immediate pointing-datum requirement arises from the desire to keep the l-foot "footprint" of the laser altimeter within a 10-foot radius of uncertainty on the ground (otherwise the 10-foot navigation accuracy would be meaningless). This is a pointing accuracy of

$$
\frac{10}{3000} \text { radian }=0.19 \text { degrees }
$$

for an altitude of 3000 feet. This is modest indeed for the proposed navigator, which has an ultimate "rule of thumb" pointing-accuracy capability of

$$
\begin{aligned}
\frac{\text { horizontal channel error }}{\text { earth radius }} & =\frac{10}{2 \times 10^{7}} \text { rad. } \\
& =2.9 \times 10^{-5} \text { degrees }
\end{aligned}
$$

In relating or tying the laser altimeter to the known pointing datum (the "p" frame fixed to the inner member of the IMU), the relaxed requirement of 0.19 degrees is the upper limit for the resolver-chain accuracy. This includes all the angular errors in the chain (i,e., from the altimeter servo-stabilized platform to the aircraft frame, deformations in the frame, and from the frame to the inner member of the IMU). 
Returning now to the high-accuracy system requirements, we note that the inertial system pays a price for its exceptional high-frequency performance. The "p" frame orientation is maintained by the measurement and removal of disturbing airframe angular rotations by the gyro triad. This measurement is, of necessity, "low-pass", in the sense that arbitrarily slow disturbing rotations cannot be discriminated against. Because of this low-pass characteristic, very low-frequency angular noise also cannot be discriminated against. This noise, called gyro drift (or gyro drift rate), is a noise-equivalent angular-rate input to the gyro, caused by irremovable physical defects, such as small shifts in the residual mass imbalance on the float of a single-degree-offreedom gyro. The term "drift" is appropriately descriptive of this noise source, since for reasonable periods of time, certainly for periods of many minutes, the noise changes monotonically rather than in an oscillatory fashion, due to its predominantly low-frequency power spectrum. In terms of modern statistical filter theory, it is more fruitful to treat the noise as an estimable state variable, than as plant noise.

The effect of gyro drift, as we shall see, is to cause both the pointing datum and the position datum to slowly drift away from the "truth", all the while maintaining their high-frequency performance. It has long been recognized that an independent source of good quality but relatively infrequent position data would complement the inertial navigator extremely well. There are many examples of systems designed with this philosophy, such as Inertial-Loran, Inertial-Omega, Inertial-Doppler, Inertial-DME, etc. The most successful of these systems treat the inertial system error as an estimable component of a state vector. The error is generally modeled by an equation which depicts the 
expected shape of the time behavior of the error, and the coefficients of the equation are estimated. This can be done by accumulating a time history of independent measurements and then obtaining a "least squares" or minimum variance estimate of the state (batch process), or recursively obtaining the minimum variance estimate (Kalman filter). If the recursive filter is used, further improvement can generally be obtained by post-run smoothing of the data. The statistical techniques employed should not obscure the fact that what is occurring is that the high-frequency performance of the inertial system and the low-frequency performance of the independent position fixer are being optimally mixed to obtain a relatively flat combined frequency characteristic at a minimum level of the total noise. The situation is depicted by Figure 2.3-1.

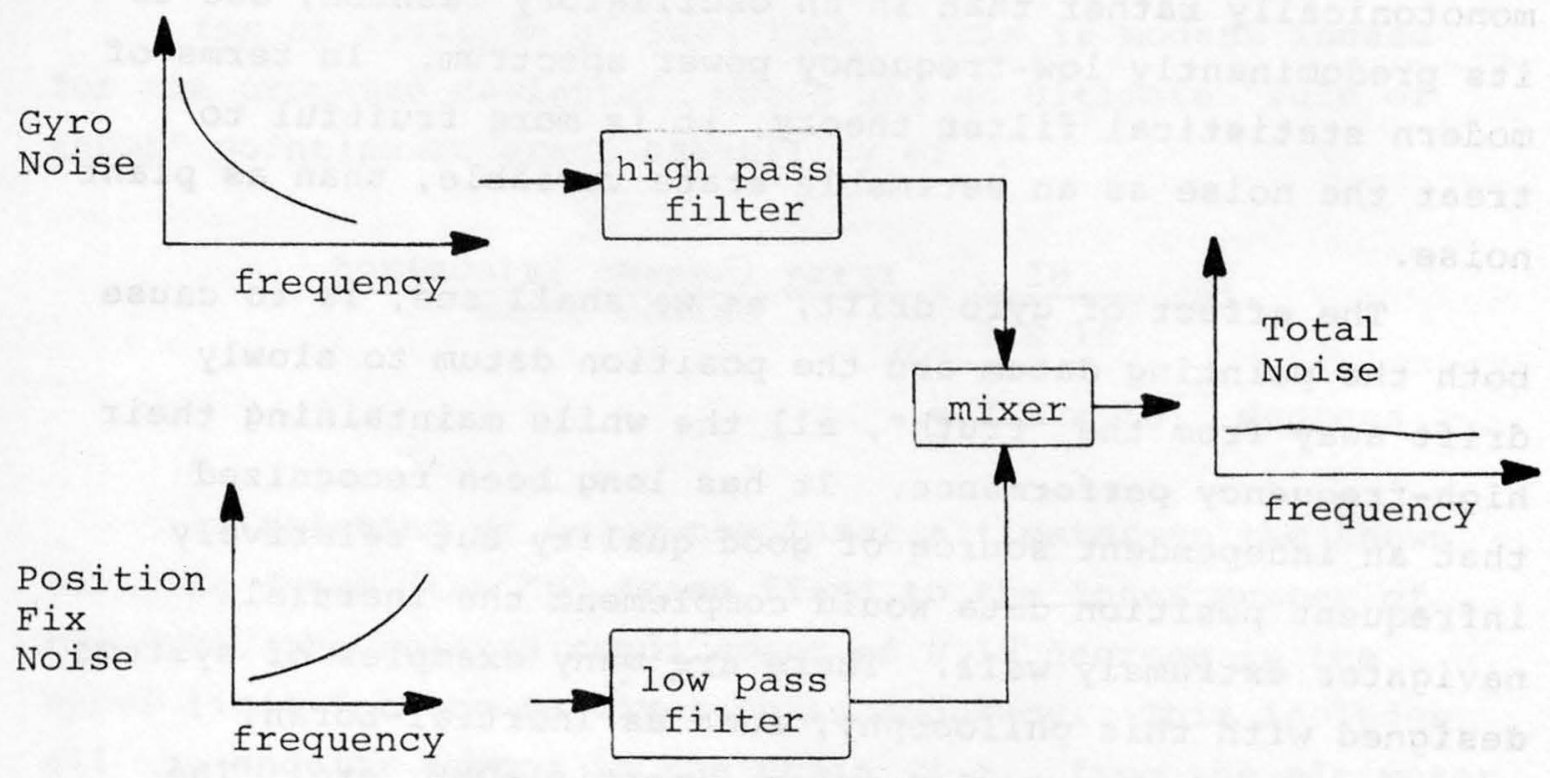

Figure 2.3-1. Frequency characteristics of IMU and position fix noise 
Looking again at the specific accuracy requirements of the system at hand, two facts become apparent:

1. The required time between independent position measurements is short.

2. The required accuracy of the position fixes is high.

The second of these, in particular, led to the choice of a laser-cooperative target tracker as the position fixing device (henceforth called the tracker). This is because laser-ranging techniques are unique in easily yielding fractional-foot accuracies consistently and over long distances. This choice looked even more attractive when design calculations indicated that the tracker could be a compact low-power device, if cooperative targets (retroreflectors) were utilized. A choice had then to be made between the exact method of deriving a position fix from the tracker. There are basically two approaches. The first of these is a completely independent position-fix determination, using range measurements to multiple targets.

(A completely non-ambiguous fix requires range to three known reference points.) This is analogous to a radio rangerange position-fix technique (such as a multiple DME fix in an aircraft).

The second possibility is to use the pointing datum provided by the inertial system (the "p" frame), and measure both range and direction in the "p" frame from the aircraft to multiple targets in sequence.

A little reflection yields the disturbing observation that the pointing datum provided by the inertial system, and with respect to which the tracker-vector measurements are being taken, is in error, due to the inextricable linking of frame-orientation errors and navigation errors in inertial systems, i.e., due to gyro drift. Thus we seem to be picking 
ourselves up by our bootstraps with this approach. That the technique is indeed a valid one is indicated by section 4.1 .4 and Appendix D. It is shown in the appendix that all the important system errors, including position errors and platform misalignments, are independently observable in the tracker data from a reference of three retroreflectors at known locations (not in a line). One of the constraints this imposes on the system design is that the ability to update platform misalignments is limited by the noise in the resolvers used in the tracker gimbals. It is not a one-toone relation, however, since the resolver noise is high frequency, and is therefore amenable to some smoothing in the estimation filter. The platform misalignment is modeled as a bias and a ramp for the period of the estimation (about 60 sec.), so uncertainties in the resolver that are uncorrelated over this interval should virtually disappear in the filter.

It is concluded that:

a) Some method of updating the airborne-instrument system from ground truth is required because of the high vertical accuracy constraint

b) Any practical laser-ranging system would require gyroscopic instruments to provide prediction signals or isolation for the tracking servos

c) The laser altimeter requires a pointing datum

d) The required highefrequency resolution is probably only obtainable from inertially derived position data

e) The use of a high quality inertial system with an integrated tracker greatly relieves the groundlogistic problem and system design by allowing all system errors to be estimated from intermittent data from a single tracker observing, in turn, three or more retroreflectors (not in a line). 
f) The proposed system combines a large number of desirable features, and provides the best probability of a successful and cost-effective design, which meets the design objectives

g) There is a large amount of growth potential and flexibility in the proposed instrument-system design, in that it provides a high quality airborne navigation reference and pointing datum for general survey-type applications, can be deployed in fixed or rotary-wing aircraft, does not require landing before completion of the survey, and minimizes the amount of ground truth required.

\subsubsection{Laser Altimeter}

The device used for measuring the distance from the airplane to the terrain surface must be capable of an accuracy of better than \pm 0.5 foot. This precludes the use of radar as a ranging device since ranging accuracy is limited to several feet and the beam divergence angle is diffraction limited to about 0.5 radians. Laser-altimeter devices, however, have been tested in aircraft at altitudes in excess of 10,000 feet and have obtained accuracies of better than 0.1 foot. As discussed in section 4.2, the laser-spot size can be easily controlled for good terrain resolution, and high signal-to-noise ratios can be obtained for all types of terrain surfaces.

The laser altimeter could conceivably be used as an aircraft position-updating device by flying directly over a surveyed retroreflector on the ground. The horizontal location of the retroreflector is known, and the altitude above this point gives the third coordinate of the aircraft. Disadvantages of this technique are discussed in section 2.3.3.

The laser-altimeter range measurements will contain 
high-frequency noise, predominantly shot noise having a flat (white noise) power spectral density. This will necessitate averaging or integrating the range measurements for periods of one to $100 \mathrm{~ms}$ (milliseconds), depending on altitude, visibility, and terrain reflectivity. Note that for an aircraft speed of 175 feet/second (120 mph) a time constant of $57 \mathrm{~ms}$ corresponds to one smoothed range measurement every ten feet along the flight path. (See section 4.2.)

A second difficulty encountered using a laser altimeter is the blocking of the laser light by vegetation. That is, the laser altimeter will measure the distance from the aircraft to the tops of trees or grass blades with occasional returns from the ground depending on the density of vegetation growth. This difficulty is also encountered in photographic map-making techniques and generally the average height of the trees is measured from the ground for correction of the terrain-elevation contours. For the aerial topographic profiling system, the average height of the vegetation may sometime need to be determined. The terrain profiles should obviously be made at points of sparsest vegetation to get a maximum number of ground returns, or in the fall or winter when density of vegetation is thin. It should be noted, however, that experiments have shown, ${ }^{1}$ that even in thickly vegetated areas, such as coniferous forests, the ground profile can still be distinguished from the trees, as shown in Fig. 2.3-2.

In brief, a laser-ranging device is the only means presently available of measuring the distance of the terrain below the aircraft to within $\pm 0.5 \mathrm{ft}$. accuracy. 


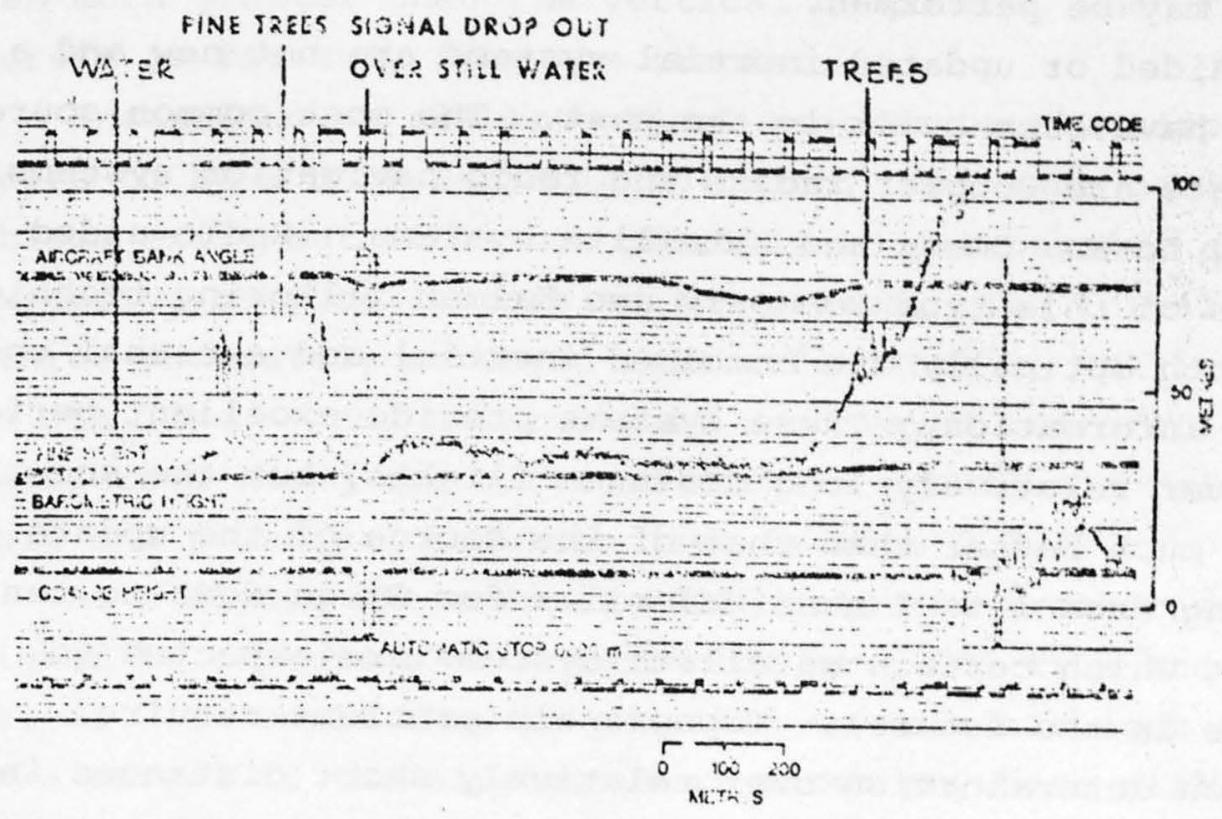

Fig. 2.3-2. Return signals from various types of terrain

\subsubsection{Laser Tracker for Updating Separate from Laser Altimeter}

It is clear that for airborne-mapping applications such as stream-valley profiling, the error propagation of an inertial system is such that external position and velocity information is required when high-accuracy system performance is desired. When system-performance requirements are of the order of magnitude of feet or fractions of a foot, the update interval must be frequent enough to keep the inertial system error growth within bounds. For stream-valley profiling the tasks of profiling and tracking cannot be time-shared by the same physical sensor, since the time intervals during which continuous profiling is performed are too long to keep the error growth bounded. A separate tracking device is 
therefore desirable so that simultaneous updating and profiling may be performed.

Aided or updated inertial systems are not new, and a number have been built in the past. The most common sources of update are Doppler radars and radio navigation systems such as Loran, Omega and sateilite systems, Radio-aided systems of this type commonly use Kalman filtering techniques to smooth optimally the combined inertial and external update system information. These systems provide excellent performance over relatively long distance flights, but the accuracy is not much better than that of the source of the update (ranging from 1 to 2 nautical miles for Omega down to tens of feet which certain satellite systems are expected to provide in the future). Topographic problems require position determination over relatively short distances in short periods of time but to a high degree of accuracy (fractions of a foot).

Electroöptical trackers have been used often in providing high-accuracy position information. The most common application is in tracking missiles or space vehicles as they leave the launch pad, Such devices obviously could be used to track the flight path of an airplane, because the airplane, or some surface on it, provides a good trackable target. Putting a tracker on the ground, however, requires the installation of an expensive system that needs power and may have to have extensive support equipment and personnel. Furthermore, in cases such as stream-valley profiling where relatively low altitude flights are often necessary in order to stay below the clouds and to follow precisely the prescribed flight paths over distances as long as 20 miles, one ground tracker would not suffice. The geometry is such that the angles subtended by the airplane with respect to the field of view of the tracker would not yield high accuracy. 
data since the horizontal component of the slant range is often much greater than the vertical component, when the aircraft is far away from the tracker.

Installing an electroöptical tracker on the airplane appears to be a more sensible solution, The same geometrical problems exist, but it is now possible to put passive and inexpensive retroreflectors on the ground, The installation of three accurately surveyed retroreflectors -- not in a straight line - and several more unsurveyed retroreflectors interspersed among the surveyed targets provides a relatively simple and practical solution (see Section 2.3.4).

At a flying altitude of 3000 feet, the tracker may be designed to capture a retroreflector at $\pm 60^{\circ}$ from the vertical. It would thus be possible to maintain lock on such a target if the airplane were flown anywhere within a two-mile diameter circle around the target. A 3000-foot flight altitude would permit staying under most clouds, but at the same time be high enough to prevent the angle of incidence from becoming excessive and causing the error in the vertical component of the slant-range measurement to become too large. Holding to the foregoing geometry the maximum slant range for the tracker would be 6000 feet, and enough laser power would still be available with a cooperative target.

To define completely the location of the airplane, slant range and angle information in two coordinates is necessary. The angle accuracy is a combination of pointing and readout accuracy. To avoid complicated angle transformations between the IMU and the tracker, which would be necessary if the tracker were separate from the IMU, and the resultant error buildup due to cascaded shaft encoders, the recommended configuration is an IMU with a tracker that can be attached to the stable member of the IMU. This provides 
base motion isolation of the tracker, and a lightweight lowinertia tracker system can be used to scan, aim and track the cooperative retroreflector, Since the stable member always has fixed geographical coordinates, regardless of the motion of the airplane, the tracker mirror angles are always referenced with respect to a lnown coordinate frame and not to the orientation of the airplane,

Another configuration one might envision is to combine the tracker with the profiler. Because both are similar electroöptical systems, one laser transmitter could be used. The transmitted beam would then be split, one beam being directed vertically downwards for profiling and the other directed to the tracking mirror. This would make the electrooptical design much more complex and costly since the problem of extreme high-accuracy angle transformations between the IMU and the tracking mirror would still exist. Furthermore, the optical power required for tracking a cooperative retroreflector is orders of magnitude less than that required for profiling, so that a relatively small and lightweight optical transmitter can be used for the tracker when it is separate from the profiler (see section 4.2.2).

The use of two separate and identical electroöptical systems where either one could be used as an altimeter or tracker would provide the most versatile configuration. This would permit both electroöptical systems to point at separate retroreflectors simultaneously, if two were in the field of view. It would also permit maintaining lock on one retroreflector for a longer period of time, while acquiring the second retroreflector. This type of configuration, of course, would be more expensive, requiring two large, heavy and high power electroöptical systems, and therefore is not recommended. 


\subsubsection{Aircraft Flight Strategies}

The selection of the aircraft flight pattern is an important part of the aerial-topographic surveying problem. By optimal updating of the inertial navigation system and proper choice of the river valley profile sequence, it is possible to ininimize the error growth in the inertial system and achieve maximum profile accuracy.

In Sections 2.4 .2 and 4.1 .2 it is shown that given a vertical accelerometer bias error, $B_{z}$, the $z$ position error grows as $\frac{1}{2} \mathrm{~B}_{\mathrm{z}} \mathrm{t}^{2}$. For $\mathrm{B}_{\mathrm{z}}$ equal to $1.0 \mathrm{\mu g}, 0.5 \mathrm{ft}$. error is reached in about three minutes. Thus, for regions with average to negligible gravity anomalies, the vertical channel of the inertial navigation system must be updated approximately every three minutes. Using a device such as the laser-tracker the horizontal channels can be corrected as well. Consequently, all types of aircraft profiling flight strategies must take into consideration the necessity of updating the guidance system at about three-minute intervals.

In this study several different methods of aerial surveying were investigated. Initially a loop flight pattern which enabled the laser altimeter to be used as a position update was studied. This involved making overlapping "loops" with standard rate turns $(3 \% / \mathrm{sec}$.$) to obtain valley$ profiles at one-quarter mile separation. In each set of loops the aircraft position could be updated by flying over a surveyed retroreflector receiving a strong range signal on the laser altimeter. This procedure requires too many surveyed retroreflectors and loses too much flight time in turning to be feasible.

A second possible surveying technique investigated consists of scanning the laser altimeter from side to side to obtain ranging data while the aircraft flies along the length of the valley. This yields diagonal profiles of the 
river valley which can be processed by computer to meet USGS error requirements. However, the difficulties encountered with this method are:

1. scanning of the laser altimeter or a large mirror quickly from side to side

2. loss of signal due to the grazing incidence of the laser radiation on the terrain surface

3. loss of signal on partially specular surfaces such as water, snow, ice and clay due to small angles of incidence

4. the difficulty in matching up diagonal profiles as the aircraft makes multiple flights up and down the valley to cover the entire valley width.

A third alternative for aerial surveying is the use of triangulation or trilateration. As discussed in Section 2.3.1, this requires either angle or range measurements to three surveyed retroreflectors simultaneously. For a twomile by twenty-mile region this requires at least 30 surveyed retroreflectors for an aircraft flying below the clouds at $3000 \mathrm{ft}$. This method is thus also not realistic. The optimal flight plan reduces the total number of ground-surveyed retroreflectors to a minimum, thereby eliminating as much ground support and advance preparation as possible. This can be achieved by locating most of the retroreflectors from the airplane so that only three reflectors would need to be surveyed on the ground, and those three should not be in a straight line. In most of the United States, networks of ground-control points offer horizontal, vertical or combined horizontal and vertical coordinate information. Therefore, very little additional ground-survey preparation would be required before flying over most local survey areas. Those retroreflectors not required to be ground surveyed would be "walked-in" and set 
up in prominent, easily visible locations such as on top of buildings or convenient land formations. These locations would be marked on a map for easy location by the lasertracker and pilot.

To implement the foregoing novel idea requires a single lengtliwise flight down the valley over the three surveyed retroreflectors and the "walked-in", unsurveyed retroreflectors. The laser-tracking system determines the position of the walked-in retroreflectors relative to the three surveyed retroreflectors and also updates or calibrates the inertial navigation system in preparation for the surveying part of the flight mission. Sightings on three successive retroreflectors allow separation of timedependent and position-dependent errors as discussed in Sections 4.1 .2 and 2.4.1. After the initial calibration exercise is completed, the flight proceeds with the necessary alternating right-and left-hand turns so that multiple crossings of the valley are made for the desired profile data. Whenever the aircraft is within line of sight (onemile radius) of a retroreflector, position updates can be made. In addition, whenever the valley profile flights intersect the initial lengthwise pass, corrections in the vertical channel are possible using the laser-altimeter data. To minimize time lost in turning the aircraft between profile runs, a turning rate of $7.5 \mathrm{deg} / \mathrm{sec}$. $(2.5$ times standard aircraft turning rate) is proposed for the two- by twenty-mile rectangular survey area. This results in a onehalf-mile diameter turn and would thus require only two interleaved sets of valley crossings up and down the valley to obtain a one-quarter-mile profile spacing. A diagram of the preferred flight plan is shown in Figures 4.1-7 and $4.1-8$.

From simulation results of severe gravity anomaly 
conditions such as those found in the Des Moines, Iowa, area it was confirmed that three surveyed retroreflectors and six walked-in retroreflectors (for the generalized 2- x 20-mile survey area) would easily meet the survey error requirements. Detailed analysis of gravity anomaly produced errors and the time dependent inertial system errors is given in sections 4.1 .2 and 4.1 .6 .

In terms of minimum ground-support time, money, personnel, and equipment, it is clear that the foregoing recommended survey-flight plan offers the best solution. The laser-tracker, laser-altimeter and inertial navigation system are all carried aboard the aircraft, and only a few inexpensive retroreflectors need be placed on the ground.

An example of a river valley, the Maury River near Lexington, Virginia, is shown in Figure 2.3-3. With three surveyed retroreflectors nearly the entire valley area of interest can be covered. One of the retroreflectors could be located at the gaging station where some coordinate position information already exists; thus, only two new surveyed positions would be needed. In such a small survey area, meeting USGS error requirements would obviously be much simpler than in a two- by twenty-mile region.

\subsection{Choice of System Elements and Optimum System} Configuration

\subsubsection{Overall System}

The overall airborne-instrument system proposed consists of three primary sensors: an inertial measurement unit (IMU), a laser tracker and a laser altimeter as outlined previously.

An airborne digital computer is the central element in the proposed system. It will accept data from the IMU, tracker and profiler and perform the necessary computations and data processing for aligning the IMU, updating position 
and velocity, calculate range and angles, and provide the storage, data logging and display functions.

The selection of the computer must be based on the assumption that it is capable of expanding as the task expands, Sufficient reserves must be available in speed, memory size and input/output capability. This will permit flexibility in planning for different types of missions other than the stream-valley profiling task to which this study was particularly directed. Reliability, maintainability and automated checkout procedures are also essential in the computer selection. Computer architecture, the instruction set and other features such as microprogramming, word length, etc., are important in the envisioned field uses where real-time computations must be made in a prescribed amount of time and to a specified accuracy.

There are a number of commercially available highreliability airborne computers that should be suitable for the preceding tasks. Airborne systems of similar complexity using inertial systems and computers in conjunction with other sensors have been built. Typical computers that would be studied for selection for the airborne-profiling system include the IBM 4ASP-1, Delco Magic-362, CDC 469, Honeywell HDC-601, ROLM 1601, Singer Kearfott SKC-2000 and Bendix BDC-910. New computers are being developed that might also be candidates at the time a final selection is made.

A block diagram of the proposed instrument system is shown in Fig, 2,4-1. The altimeter and tracker will have local data processors, probably in the form of microprocessors, that will perform averaging and smoothing of the range and angle data. The smoothed data will then be sent at a relatively low speed to the main processor.

A control and display subsystem will provide the means for the operator to control and monitor the modes of 


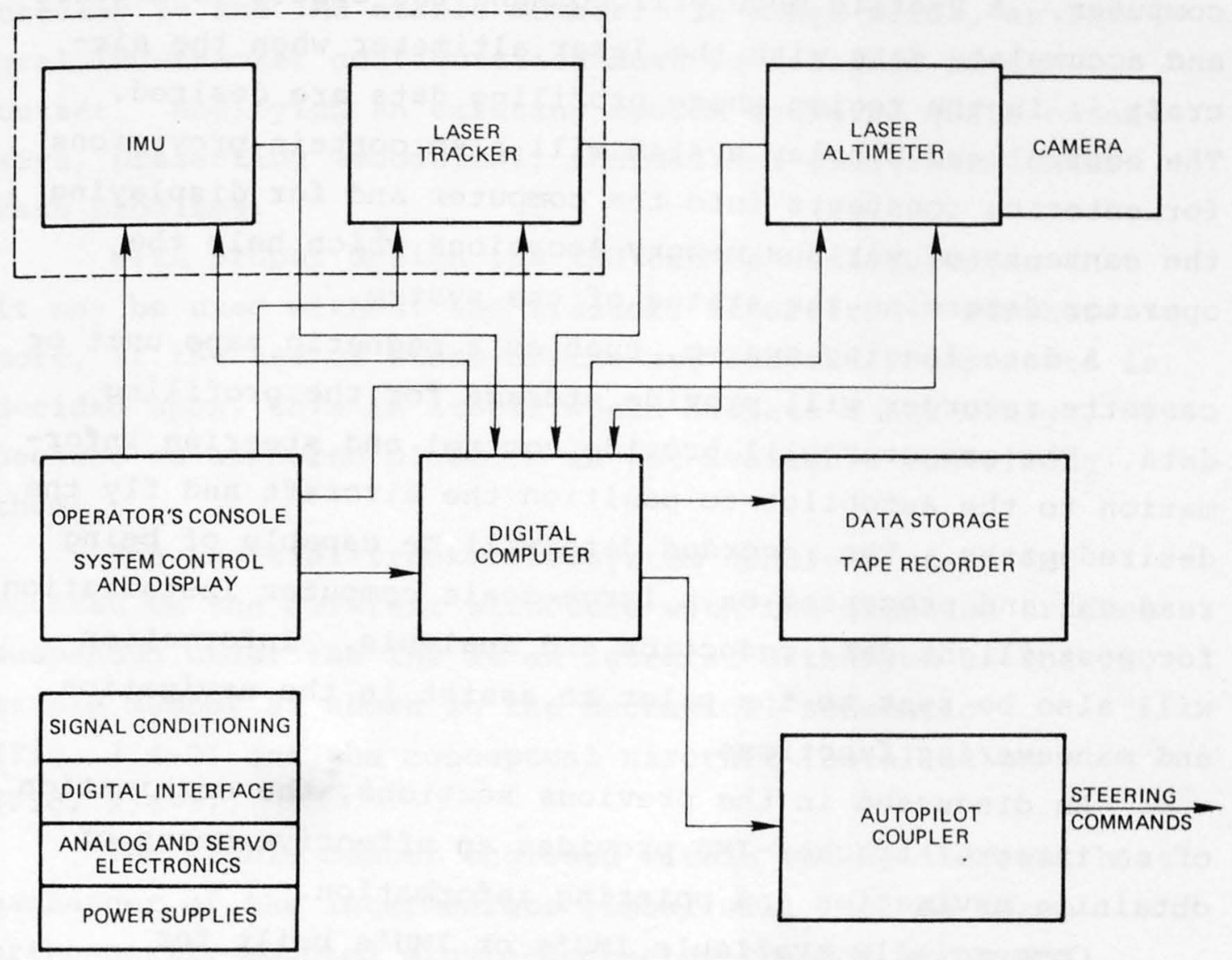

Fig. 2,4-1. Airborne profiling system 
operation and observe the status of the system. The operating modes include a Standby Warm-up mode, IMU Align, Calibrate and Normal Flight. In addition, a search mode for the tracker will enable the operator to command the computer to acquire a retroreflector in those cases where it is desired to override the automatic search and track sequencing of the computer. A profile mode will command the system to acquire and accumulate data with the laser altimeter when the aircraft is in the region where profiling data are desired. The control and display system will also contain provisions for entering constants into the computer and for displaying the contents of various memory locations which help the operator determine the status of the system.

A data-logging system, such as a magnetic tape unit or cassette recorder, will provide storage for the profiling data. The computer will provide control and steering information to the autopilot to position the aircraft and fly the desired paths. The recorded data will be capable of being read out and processed on a large-scale computer installation for post-flight data reduction and analysis. Information will also be sent to the pilot to assist in the navigation and maneuvering functions.

As discussed in the previous sections, the combination of an integral tracker-IMU provides an effective means of obtaining navigation and pointing information.

Commercially available IMU's or IMU's built for military applications, were considered for this proposed instrument system. Even if an IMU could be found with gyros and accelerometers of the required accuracy and stability, the problem of coupling it to the tracker would still exist. Precision gimbal angle readout devices such as inductosyns with 5-to 10-arc second accuracy would have to be mounted on the gimbals with considerable mechanical modifications. The 
integral IMU-tracker design requires a gimbal orientation of azimuth, pitch and roll (from the innermost member to the outside gimbal) in order to provide the correct axes for pointing the tracker. Any other orientation would require coordinate transformations involving precision gimbal angle readout devices, eliminating the advantages of coupling the tracker to the IMU stable member. In other words, an integral IMU-tracker configuration must be designed at the outset. Modifying an existing system would be quite extensive, presenting mechanical, thermal and electronic interface problems.

With proper design the IMU can be configured so that it may be used without the tracker, if desired. Furthermore, if the use of state-of-the-art inertial components is decided upon, this in itself would dictate a new design, because no airborne platform is yet available containing them.

The inertial-tracker subsystem consists of an IMU mounted on the aircraft structure with the gimbaled tracker suspended under the IMU as an integral extension of the IMU stable member as shown in the mechanical schematic (Fig. 2.4-2) and the conceptual aircraft installation (Fig. 2.4-3).

The stable member enclosed within the cylindrical heat exchanger of the intermediate gimbal will include three orthogonally mounted single-degree-of-freedom gyros and three specific force receivers (accelerometers). Precision electronics, such as temperature controller and signal pickoff electronics, will be located on the stable member. The stable member tracks local vertical maintaining a true north reference by means of three isolation axes (roll, pitch and azimuth). It will have its own temperature-control system, using a combination of axial flow fans and resistance heaters 


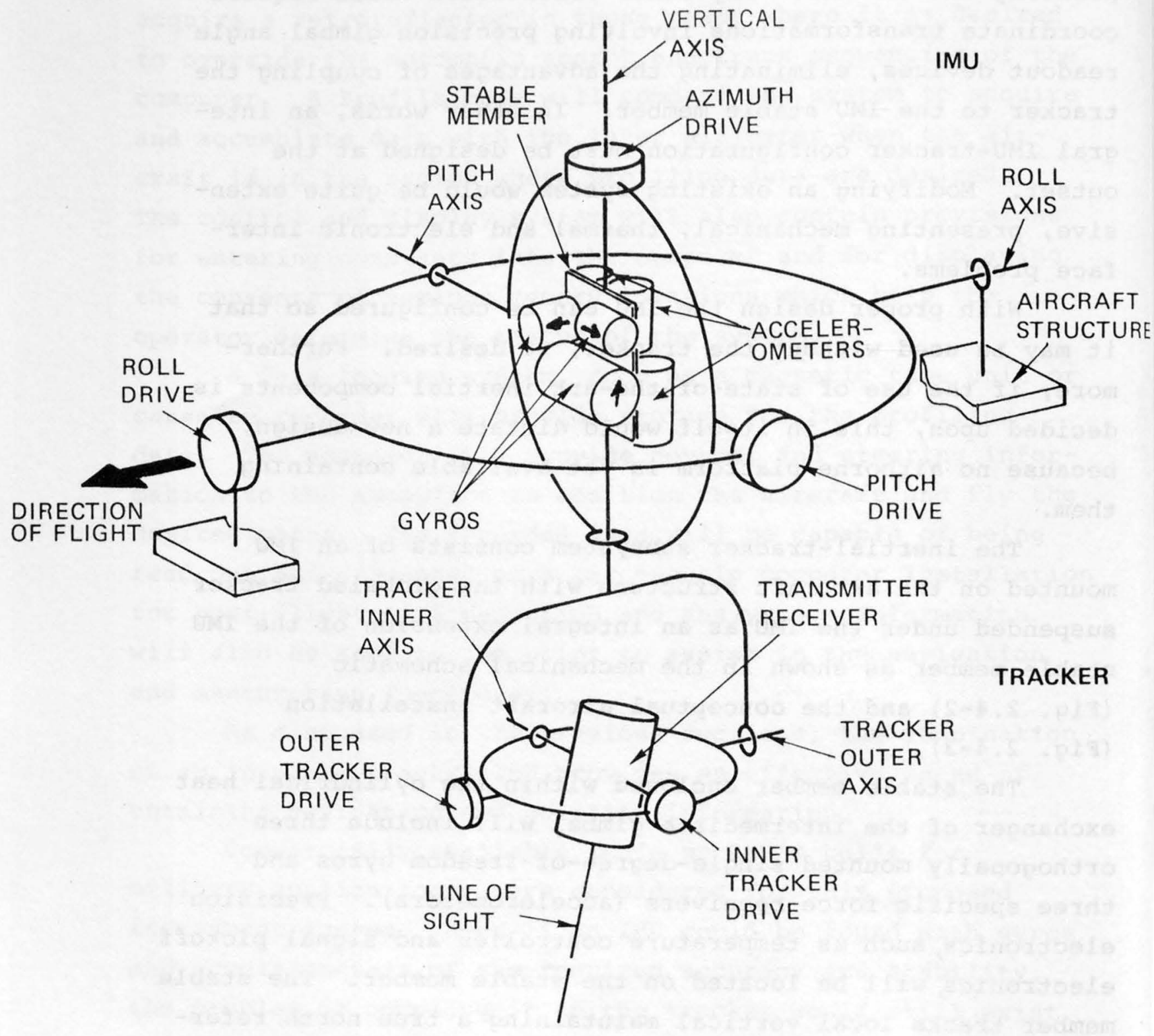

Fig. 2.4-2. Mechanical schematic of IMU and laser-tracker gimbaling 


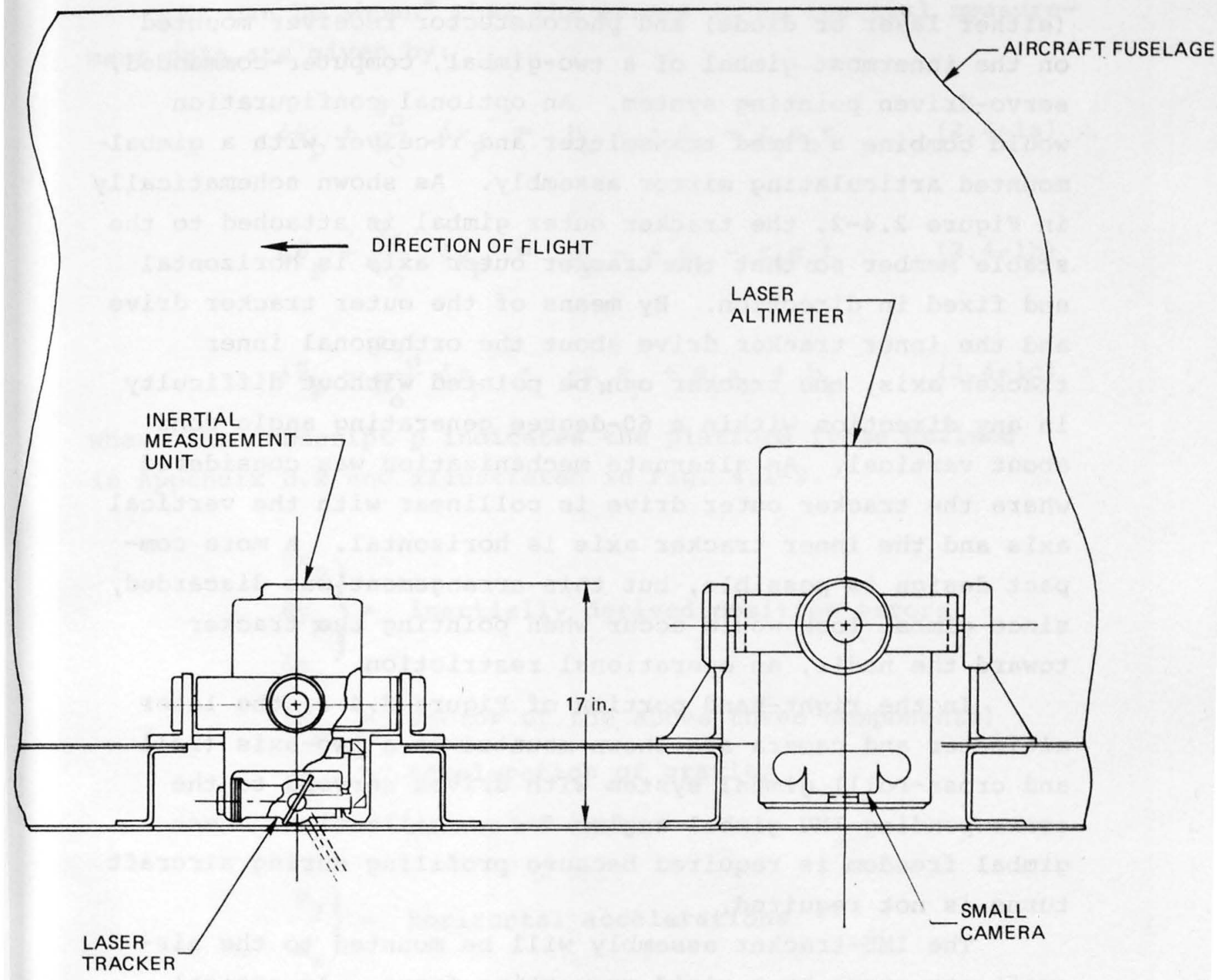

Fig. 2.4-3. Conceptual disposition of instrument package in aircraft 
to maintain the proper thermal environment for the inertial instruments. Cabin air will be used as the ultimate heat sink and no auxiliary refrigeration will be necessary.

The tracker assembly consists of an optical transmitter (either laser or diode) and photodetector receiver mounted on the innermost gimbal of a two-gimbal, computer-commanded, servo-driven pointing system. An optional configuration would combine a fixed transmitter and receiver with a gimbalmounted articulating mirror assembly. As shown schematically in Figure 2.4-2, the tracker outer gimbal is attached to the stable member so that the tracker outer axis is horizontal and fixed in direction. By means of the outer tracker drive and the inner tracker drive about the orthogonal inner tracker axis, the tracker can be pointed without difficulty in any direction within a 60-degree generating angle cone about vertical. An alternate mechanization was considered where the tracker outer drive is collinear with the vertical axis and the inner tracker axis is horizontal. A more compact design is possible, but this arrangement was discarded, since gimbal lock would occur when pointing the tracker toward the nadir, an operational restriction.

In the right-hand portion of Figure 2.4-3, the laser altimeter and camera are shown mounted on a two-axis (roll and cross-roll) gimbal system with drives servoed to the corresponding IMU gimbal angles for stabilization. Less gimbal freedom is required because profiling during aircraft turns is not required.

The IMU-tracker assembly will be mounted to the aircraft structure by a rigid supporting frame. An optical viewing port (approximately 18 inches in diameter) will be installed in the bottom of the aircraft to accommodate the tracker field of view. A similar window will be required for the laser altimeter. 


\subsubsection{Criteria for Selection of Inertial Components}

\subsubsection{Analysis of Inertial System Error Growth}

It can be shown ${ }^{2}$ that the errors in an inertial measurement unit are given by:

$$
\begin{aligned}
& \delta \ddot{x}_{p}+\frac{g_{0}}{R_{o}} \delta x_{p}=b_{x}+a_{y} \psi_{z}-\varepsilon_{y} g_{o} t \quad(2.4-1 a) \\
& \delta \ddot{y}_{p}+\frac{g_{o}}{R_{o}} \delta y_{p}=b_{y}-a_{x} \psi_{z}-\varepsilon_{x} g_{o} t \\
& \delta \ddot{z}_{p}-\frac{2 g_{o}}{R_{o}} \delta z_{p}=-\psi_{x} a_{y}+\psi_{y} a_{x}+b_{z}
\end{aligned}
$$

where the subscript $p$ indicates the platform frame defined in Appendix D.2 and illustrated in Fig. 4.1-3.

$$
\begin{aligned}
& \left.\begin{array}{c}
\delta x_{p} \\
\delta y_{p} \\
\delta z_{p}
\end{array}\right\}=\text { inertially derived position errors } \\
& \left(\delta \bar{x}_{p}=\right.\text { vector of the above three components) } \\
& g_{0}=\text { acceleration of gravity } \\
& \mathrm{R}_{\mathrm{O}}=\text { radius of earth } \\
& \left.a_{x} a_{y}\right\}=\text { horizontal accelerations } \\
& \left.\begin{array}{c}
\varepsilon_{\mathrm{x}} \\
\varepsilon_{\mathrm{y}}
\end{array}\right\}=\text { horizontal gyro drift }
\end{aligned}
$$




$$
\begin{aligned}
& \left.\begin{array}{l}
\psi_{\mathrm{x}} \\
\psi_{\mathrm{y}}
\end{array}\right\}=\text { platform misalignment angles } \\
& (\Psi=\text { vector of the above three components }) \\
& \mathrm{t}=\mathrm{time} \\
& \mathrm{b} \\
& \left.\begin{array}{l}
b_{x} \\
b \\
b
\end{array}\right\}=\begin{array}{l}
\text { accelerometer bias error (lst order effect } \\
\text { of } b_{x}, b_{y} \text { and } b_{z} \text { assumed removed during } \\
\text { self-leveling operation) }
\end{array}
\end{aligned}
$$

The second term on the left-hand side of Equations $(2.4-1 a),(2.4-1 b)$ and $(2,4-1 c)$ is called the "feedback" term. This gives rise to the schuler oscillation in the horizontal channels and exponential error growth in the vertical channel. The constant $g_{0} / R_{0}$ is given by

$$
\frac{g_{O}}{R_{0}}=\frac{32.2 \mathrm{ft} / \mathrm{sec}^{2}}{2.09 \times 10^{7} \mathrm{ft}}=1.54 \times 10^{-6} \mathrm{sec}^{-2}
$$

From which the "Schuler" frequency is given by

$$
\begin{aligned}
\omega_{S}=\sqrt{\frac{g_{O}}{R_{O}}} & =1.24 \times 10^{-3} \mathrm{rad} / \mathrm{sec} \\
& =1.97 \times 10^{-4} \mathrm{~Hz} .
\end{aligned}
$$

An important number is the Schuler period, $\frac{1}{\omega_{S}}$, given by:

$$
\frac{1}{\omega_{s}}=5060 \mathrm{sec} .84 \mathrm{~min} \text {. }
$$

Thus the oscillatory solution implied by (la) and (lb) has a period of $5060 \mathrm{sec}$. 
Rewrite Eq. (2.4-1) as

$$
\begin{aligned}
& \delta \ddot{x}_{p}+\omega_{s}^{2} \delta x_{p}=K X_{0}+K x_{l} t \\
& \delta \ddot{y}_{p}+\omega_{s}^{2} \delta y_{p}=K Y_{0}+K Y_{l} t \\
& \delta \ddot{z}_{p}-2 \omega_{s}^{2} \delta z_{p}=K Z_{0}
\end{aligned}
$$

for which the zero initial condition solutions are:

$$
\begin{aligned}
& \delta x_{p}=\frac{K X_{O}}{\omega_{s}^{2}}\left(1-\cos \omega_{s} t\right)+\frac{K X_{1}}{\omega_{s}^{3}}\left(\omega_{s} t-\sin \omega_{s} t\right)(2.4-3 a) \\
& \delta y_{p}=\frac{K Y o}{\omega_{s}^{3}}\left(1-\cos \omega_{s} t\right)+\frac{K Y_{1}}{\omega_{s}^{3}}\left(\omega_{s} t-\sin \omega_{s} t\right)(2 \cdot 4-3 b) \\
& \delta z_{p}=\frac{K Z_{O}}{2 \omega_{s}^{2}}\left(\cosh \sqrt{2} \omega_{s} t-1\right)
\end{aligned}
$$

(Zero initial conditions are not necessary, but are used here to illustrate the procedure.)

It is now possible to separate the solution into two distinct time domains. First we note that

$$
\begin{aligned}
& \cos \omega_{s} t=1-\frac{\left(\omega_{s} t\right)^{2}}{2 !}+\frac{\left(\omega_{s} t\right)^{4}}{4 !}-\cdot \cdot(2.4-4 a) \\
& \sin \omega_{s} t=\omega_{s} t-\frac{\left(\omega_{s} t\right)^{3}}{3 !}+\frac{\left(\omega_{s} t\right)^{5}}{5 !}-\cdot \cdot(2.4-4 b) \\
& \cosh \left(\sqrt{2} \omega_{s} t\right)=1+\frac{\left(\sqrt{2} \omega_{s} t\right)^{2}}{2 !}+\frac{\left(\sqrt{2} \omega_{s} t\right)^{4}}{4 !}+(2.4-4 c)
\end{aligned}
$$


Substituting $(2.4-4)$ back into (2.4-3) we note that, for $t<\omega_{s}$

$$
\begin{aligned}
& \delta x_{p} \simeq \frac{K X_{o}}{2} t^{2}+\frac{K X_{1}}{6} t^{3} \\
& \delta y_{p} \simeq \frac{K Y_{o}}{2} t^{2}+\frac{K Y_{1}}{6} t^{3} \\
& \delta z_{p} \simeq \frac{K z_{O}}{2} t^{2}
\end{aligned}
$$

Equation (2.4-5) is the "short term" error behavior, and is of most interest for the USGS application, where relatively frequent laser updates (compared to the Schuler period) are envisioned.

A significant fact is that, comparing (2.4-2) and $(2.4-1)$ we see that

$$
\begin{aligned}
& \mathrm{KX}_{1}=\varepsilon_{\mathrm{Y}^{\mathrm{g}}} \\
& \mathrm{KY}_{1}=\varepsilon_{\mathrm{x}^{\mathrm{g}}}
\end{aligned}
$$

That is, gyro drift in the short term causes a $t^{3}$ error growth in the horizontal channels. Accelerometer bias, on the other hand, gives rise to a $t^{2}$ error growth in the horizontal channel and in the vertical channel.

For the sake of completeness, we can note that, for $t>\omega_{s}$, the "long term" solutions can be obtained

$$
\delta x_{p} \simeq \frac{k x_{1}}{\omega_{s}^{2}} t
$$




$$
\begin{aligned}
& \delta y_{p} \simeq \frac{K Y_{1}}{\omega_{s}^{2}} t \\
& \delta z_{p} \simeq \frac{K Z_{o}}{4 \omega_{s}^{2}} e^{\left(\sqrt{2} \omega_{s} t\right)}
\end{aligned}
$$

which are the classical "rule of thumb" performance equations for inertial navigators, with gyro drift giving rise to an error growth in the horizontal channels proportional to time, and the vertical channel exhibiting strong instability.

One could neglect the gravity feedback in the vertical channel, in which case $(2.4-6 c)$ becomes

$$
\delta z_{p}=\frac{K z_{o}}{2} t^{2}
$$

We note that the feedback terms have no effect on the short-term solution (2,4-5) due, of course, to the length of the Schuler period. Thus the short-term solutions could have been obtained by simply eliminating the feedback terms from the differential equation (2.4-2) and double integrating for the solution $(2,4-5)$.

Rewriting (2.4-5a) and (2.4-5b) and neglecting the relatively unimportant constant terms, we get, neglecting the acceleration dependent errors,

$$
\left|\delta x_{p}\right|=\frac{b_{x}}{2} t^{2}+\frac{\varepsilon g_{o}}{6} t^{3}
$$

where

$$
\begin{aligned}
& \mathrm{b}_{\mathrm{x}}=\text { residual horizontal accelerometer bias } \\
& \varepsilon=\text { horizontal channel gyro drift } \\
& g_{0}=\text { acceleration of gravity }
\end{aligned}
$$


In order to get a feel for the relative contributions of accelerometer bias and gyro drift to the horizontal channel errors, we use (2.4-8) to construct Fig. 2.4-4. The diagonal lines represent equal contribution to $\delta x$ by the accelerometer bias error (abscissa) in micro-gravity ( $\mu g$ ) units and gyro drift (ordinate) in milli-earth rate units (meru). Because of the stronger time dependence of gyro drift-induced errors, the longer the time, the more the burden shifts to the gyro, as shown. The three lines drawn represent $t=60 \mathrm{sec}, 200 \mathrm{sec}$. and $600 \mathrm{sec}$. Sixty seconds represent about the time that the tracker would remain "locked on" to a retroreflector from a direct overhead pass at 3000 feet; 600 seconds represent an extreme upper limit in time between tracking of retroreflectors. One of the important factors we obtain from this plot is the high requirement on short-ierm stability (and resolution) of the horizontal accelerometers.

Two examples of the usage of the graph follow:

a) Short-term accelerometer bias stability is quoted as $10 \mu \mathrm{g}$ and short-term gyro drift stability is quoted as 0.01 meru. We wish to know what the uncompensated position error growth is during a tracking interval (60 sec.); and which instrument has the dominant effect, assuming zero initial errors. We find the intersection of $10 \mu \mathrm{g}$ and 0.01 meru and note that it is below the $60-\mathrm{sec}$. Iine, so that the accelerometer bias error effect dominates. The error is simply $\frac{1}{2} a t^{2}$, since the $t^{3}$ contribution can be neglected. Hence

$$
\delta x_{p}=\frac{1}{2}(32.2)\left(10^{-5}\right)(60)^{2}=0.6 \mathrm{ft} .
$$




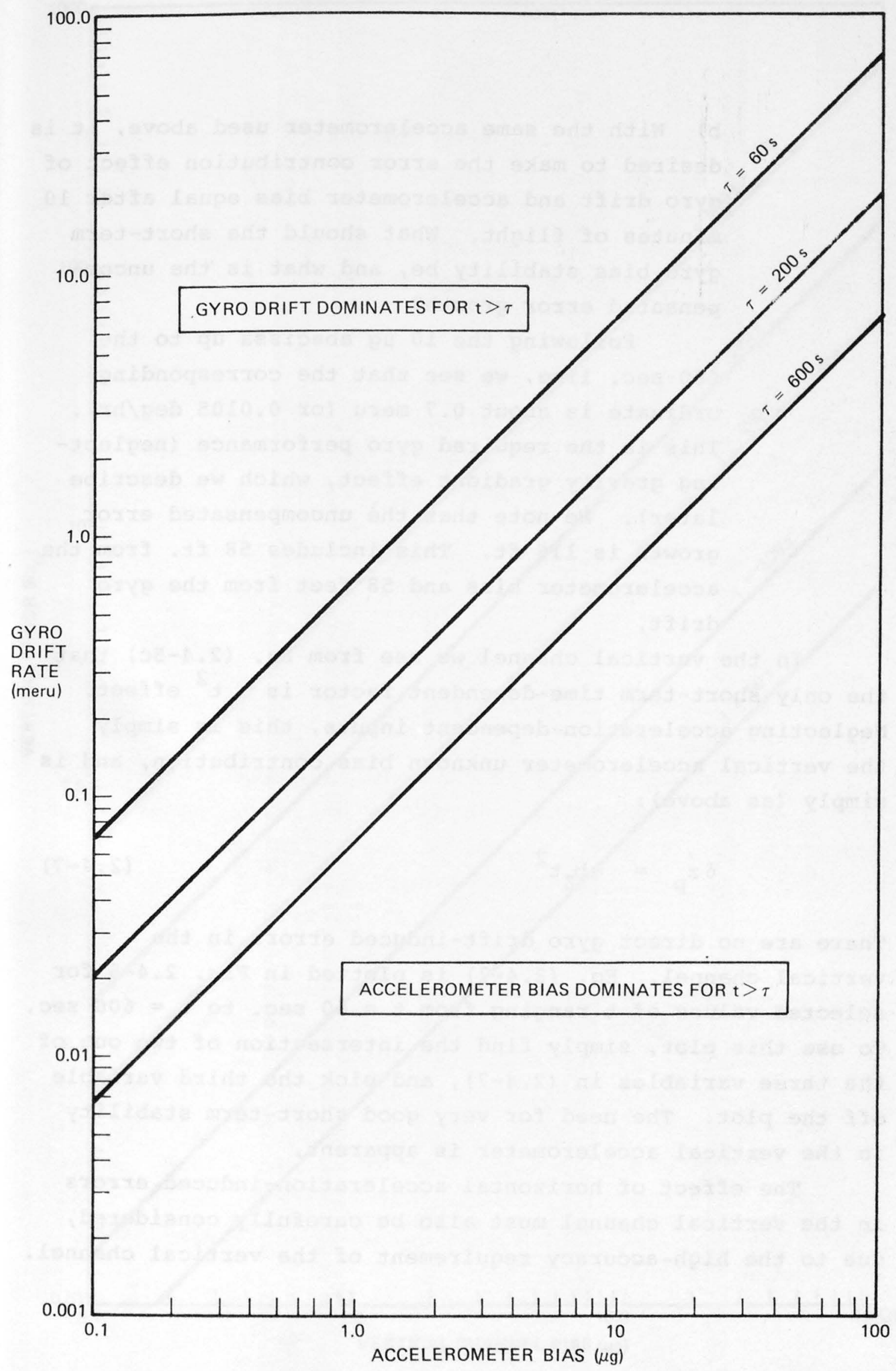

Fig. 2.4-4. Relative contributions of gyros and accelerometers to horizontal errors 
b) With the same accelerometer used above, it is desired to make the error contribution effect of gyro drift and accelerometer bias equal after 10 minutes of flight. What should the short-term gyro bias stability be, and what is the uncompensated error growth?

Following the $10 \mu \mathrm{g}$ abscissa up to the 600-sec. line, we see that the corresponding ordinate is about 0.7 meru (or $0.0105 \mathrm{deg} / \mathrm{hr}$ ). This is the required gyro performance (neglecting gravity gradient effect, which we describe later). We note that the uncompensated error growth is $116 \mathrm{ft}$. This includes $58 \mathrm{ft}$. from the accelerometer bias and 58 feet from the gyro drift.

In the vertical channel we see from Eq. (2.4-5c) that the only short-term time-dependent factor is a $t^{2}$ effect. Neglecting acceleration-dependent inputs, this is simply the vertical accelerometer unknown bias contribution, and is simply (as above):

$$
\delta z_{p}=\frac{1}{2} b_{z} t^{2}
$$

There are no direct gyro drift-induced errors in the vertical channel. Eq. $(2.4-7)$ is plotted in Fig. 2.4-5 for selected values of $t$ ranging from $t=60 \mathrm{sec}$, to $t=600 \mathrm{sec}$. To use this plot, simply find the intersection of two out of the three variables in $(2.4-7)$, and pick the third variable off the plot. The need for very good short-term stability in the vertical accelerometer is apparent.

The effect of horizontal acceleration-induced errors in the vertical channel must also be carefully considered, due to the high-accuracy requirement of the vertical channel. 


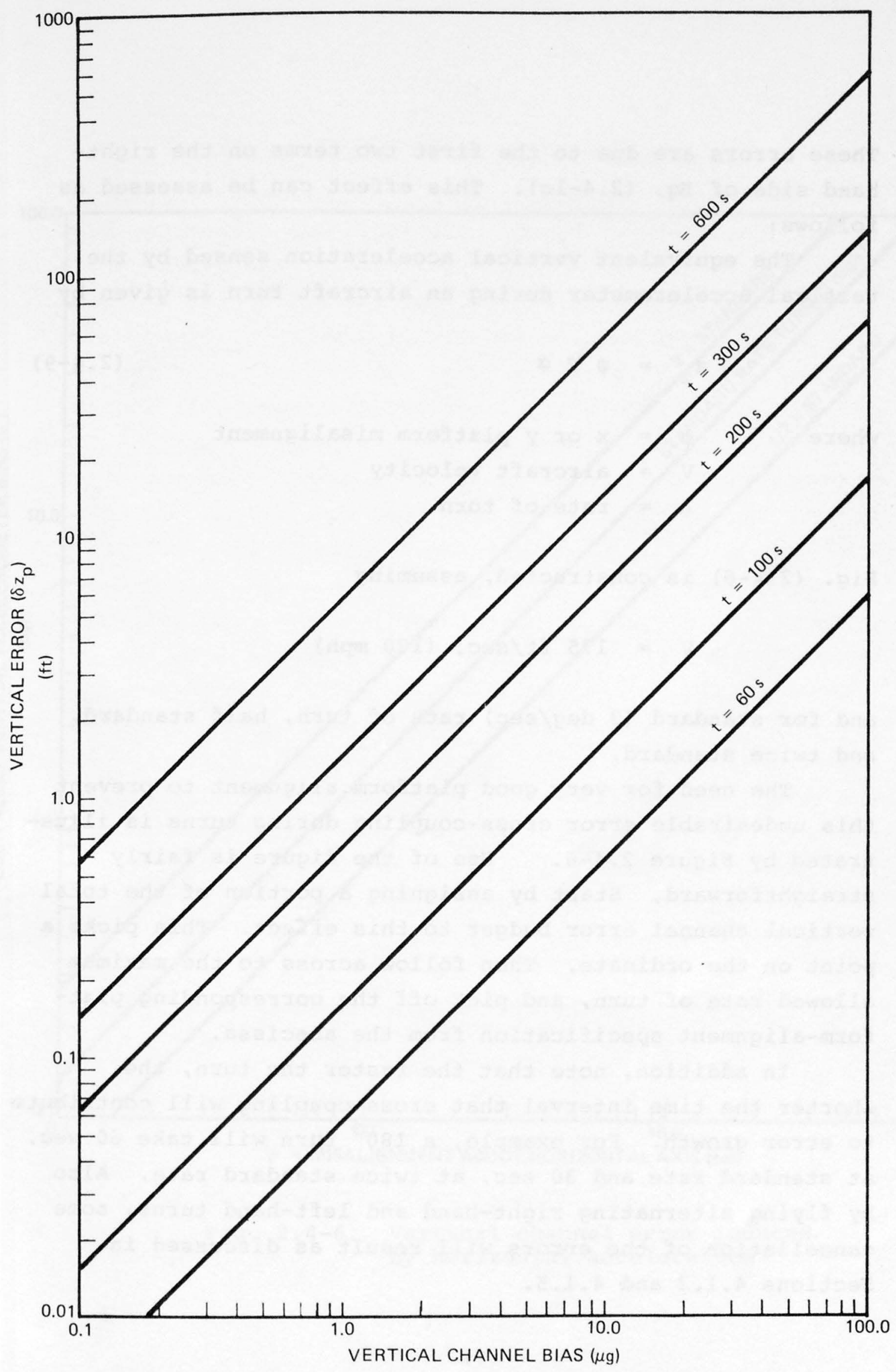

Fig. 2.4-5. Vertical channel error due to vertical accelerometer bias 
These errors are due to the first two terms on the righthand side of Eq. (2.4-1C). This effect can be assessed as follows :

The equivalent vertical acceleration sensed by the vertical accelerometer during an aircraft turn is given by

$$
a_{z}=\psi v \alpha
$$

where

$$
\begin{aligned}
\psi & =\mathrm{x} \text { or } \mathrm{y} \text { platform misalignment } \\
\mathrm{V} & =\text { aircraft velocity } \\
\alpha & =\text { rate of turn }
\end{aligned}
$$

Fig. $(2.4-6)$ is constructed, assuming

$$
\mathrm{V}=175 \mathrm{ft} / \mathrm{sec},(120 \mathrm{mph})
$$

and for standard ( $3 \mathrm{deg} / \mathrm{sec}$ ) rate of turn, half standard, and twice standard.

The need for very good platform alignment to prevent this undesirable error cross-coupling during turns is illustrated by Figure 2.4-6. Use of the figure is fairly straightforward. Start by assigning a portion of the total vertical channel error budget to this effect. This picks a point on the ordinate. Then follow across to the maximum allowed rate of turn, and pick off the corresponding platform-alignment specification from the abscissa.

In addition, note that the faster the turn, the shorter the time interval that cross-coupling will contribute to error growth. For example, a $180^{\circ}$ turn will take $60 \mathrm{sec}$. at standard rate and $30 \mathrm{sec}$. at twice standard rate. Also by flying alternating right-hand and left-hand turns, some cancellation of the errors will result as discussed in Sections $4.1,2$ and $4.1,5$. 


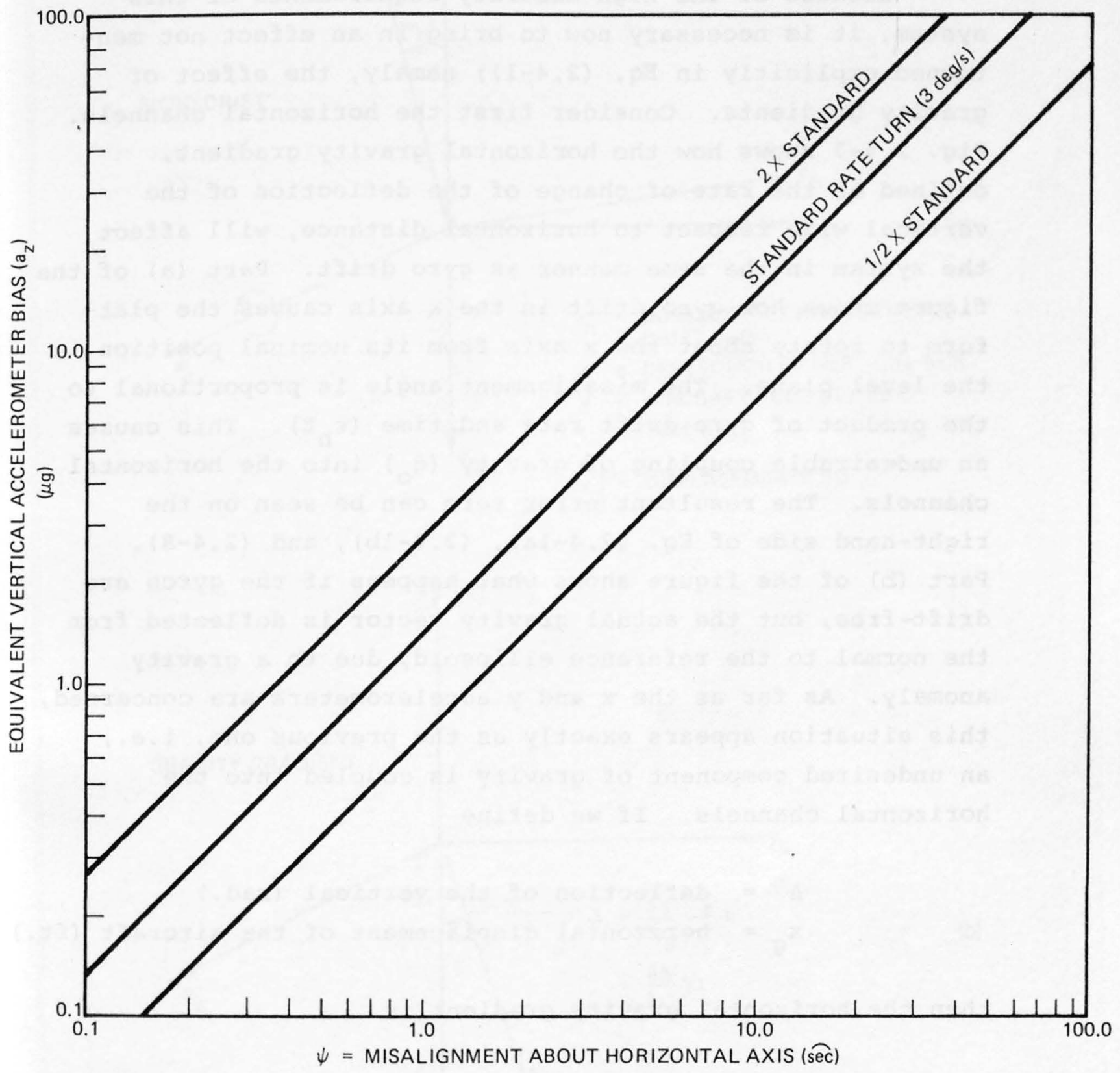

Fig. 2.4-6. Vertical channel error induced by horizontal acceleration 


\subsubsection{Gravity Anomaly Produced Errors}

Because of the high-accuracy requirements of this system, it is necessary now to bring in an effect not mentioned explicitly in Eq. (2.4-1); namely, the effect of gravity gradients. Consider first the horizontal channels. Fig. 2.4-7 shows how the horizontal gravity gradient, defined as the rate of change of the deflection of the vertical with respect to horizontal distance, will affect the system in the same manner as gyro drift. Part (a) of the figure shows how gyro drift in the $x$ axis causes the platform to rotate about the $\mathrm{x}$ axis from its nominal position in the level plane, The misalignment angle is proportional to the product of gyro drift rate and time $\left(\varepsilon_{h} t\right)$. This causes an undesirable coupling of gravity $\left(g_{0}\right)$ into the horizontal channels. The resultant error term can be seen on the right-hand side of Eq. (2.4-la), (2.4-1b), and (2.4-8), Part (b) of the figure shows what happens if the gyros are drift-free, but the actual gravity vector is deflected from the normal to the reference ellipsoid, due to a gravity anomaly. As far as the $x$ and $y$ accelerometers are concerned, this situation appears exactly as the previous one, i.e., an undesired component of gravity is coupled into the horizontal channels. If we define

$$
\begin{aligned}
& \Delta=\text { deflection of the vertical (rad.) } \\
& x_{g}=\text { horizontal displacement of the aircraft (ft.) }
\end{aligned}
$$

then the horizontal gravity gradient is

$$
\frac{\partial \Delta}{\partial x_{g}} \text {; and the angular misalignment of the gravity }
$$



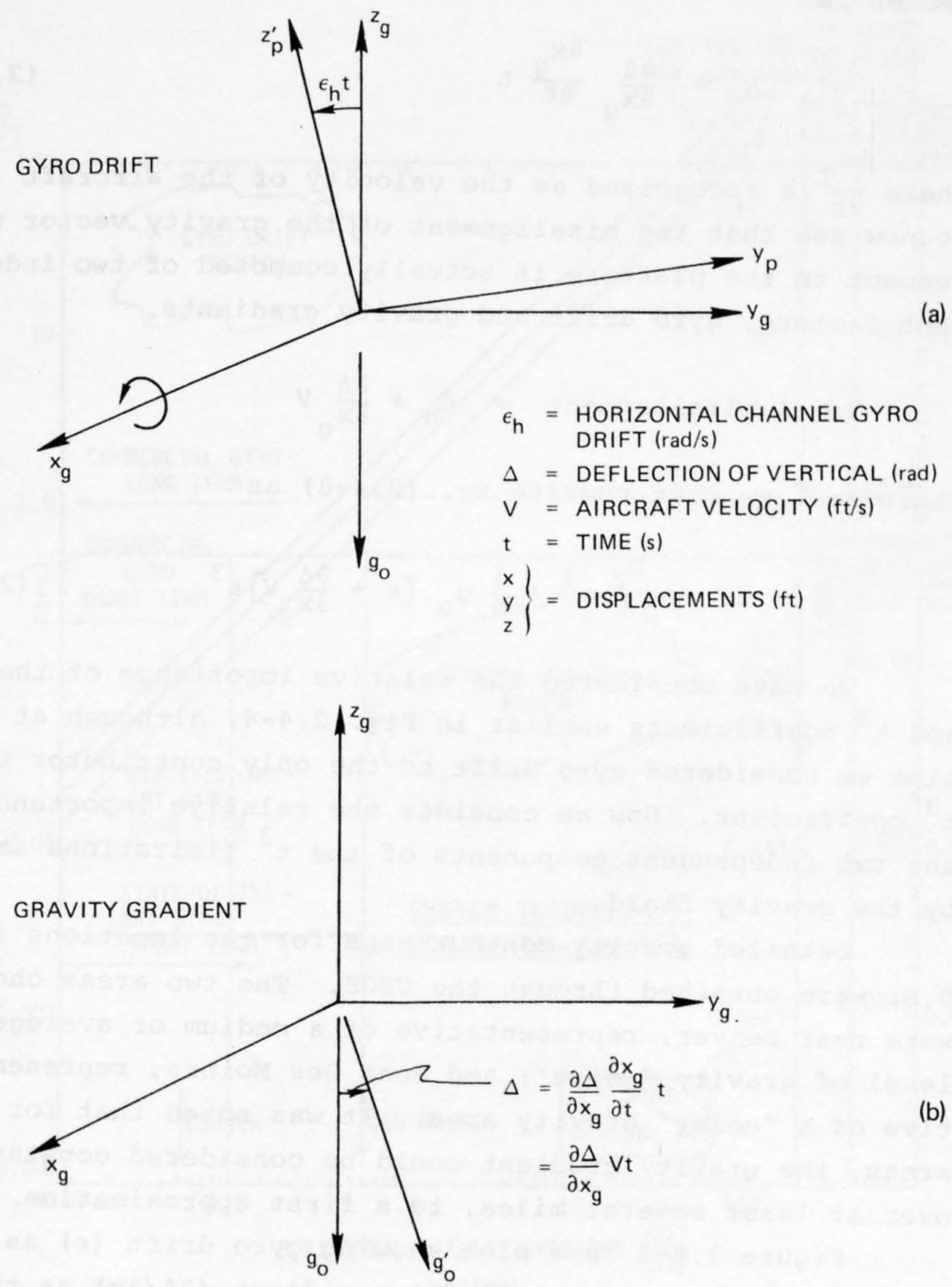

(b)

Fig. 2.4-7. Relation of gravity gradient to gyro drift 
vector is

$$
\Delta=\frac{\partial \Delta}{\partial x_{g}} \frac{\partial x_{g}}{\partial t} t
$$

where $\frac{\partial x_{g}}{\partial t}$ is recognized as the velocity of the aircraft (V). We now see that the misalignment of the gravity vector with respect to the platform is actually composed of two independent factors, gyro drift and gravity gradients.

$$
\text { Total misalignment }=\varepsilon_{h}+\frac{\partial \Delta}{\partial x_{g}} V
$$

Therefore, we must rewrite Eq, $(2,4-8)$ as

$$
\delta r=\frac{b_{z}}{2} t^{2}+\frac{1}{6} g_{0}\left[\varepsilon+\frac{\partial \Delta}{\partial x_{g}} v\right] t^{3}
$$

We have considered the relative importance of the $t^{2}$ and $t^{3}$ coefficients earlier in Fig. 2.4-4, although at that time we considered gyro drift as the only contributor to the $t^{3}$ coefficient. Now we consider the relative importance of the two independent components of the $t^{3}$ limitations imposed by the gravity field.

Detailed gravity-contour maps for two locations in the U.S. were obtained through the USGS. The two areas chosen were near Denver, representative of a medium or average level of gravity "noise"; and near Des Moines, representative of a "noisy" gravity area. It was noted that for both areas, the gravity gradient could be considered constant over at least several miles, to a first approximation.

Figure 2.4-8 is a plot showing gyro drift $(\varepsilon)$ as the ordinate, and horizontal gravity gradient $(\partial \Delta / \partial x)$ as the abscissa, with the aircraft velocity as a parametric line. Along the line corresponding to the aircraft velocity, the 


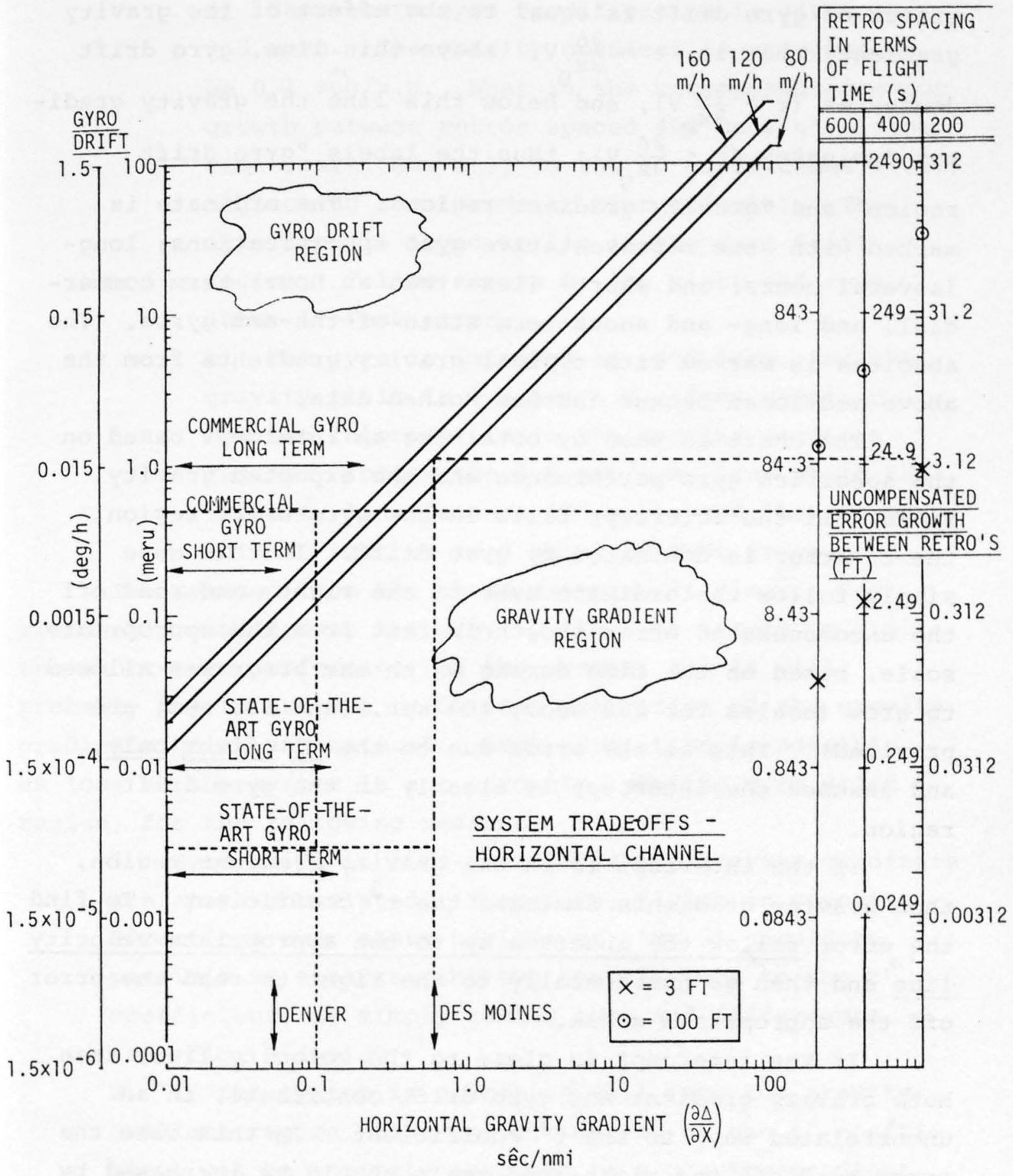

Fig. 2.4-8. Gyro drift versus horizontal gravity gradient at three velocities of aircraft 
effect of gyro drift is equal to the effect of the gravity gradient; that is, $\varepsilon=\frac{\partial \Delta}{\partial x_{g}} \mathrm{~V}$. Above this line, gyro drift dominates $\left(\varepsilon>\frac{\partial \Delta}{\partial x_{g}} V\right)$, and below this line the gravity gradient dominates $\left(\varepsilon<\frac{\partial \Delta}{\partial x_{g}} V\right)$; thus the labels "gyro drift region" and "gravity gradient region". The ordinate is marked with some representative gyro specifications: long(several hours) and short- (less than an hour) term commercial, and long- and short-term state-of-the-art gyros. The abscissa is marked with typical gravity gradients from the above-mentioned Denver and Des Moines data.

The chart is used by obtaining an intercept based on the specified gyro performance and the expected gravity field. If the intercept falls in the gyro drift region, the $t^{3}$ error is dominated by gyro drift. In this case simply follow the ordinate over to the right, and read off the uncompensated error growth in feet from the appropriate scale, based on the time during which the error was allowed to grow (scales for $200 \mathrm{sec}, 400 \mathrm{sec}$, and $600 \mathrm{sec}$. are provided). This is the error due to the $t^{3}$ effect only, and assumes the intercept is clearly in the gyro drift region.

If the intercept is in the gravity gradient region, then gravity gradients dominate the $t^{3}$ coefficient. To find the error follow the abscissa up to the appropriate velocity line and then go horizontally to the right to read the error off the appropriate scale.

If the intercept is close to the velocity line, then both gravity gradient and gyro drift contribute, in an uncorrelated way, to the $t^{3}$ coefficient. In this case the error read off the right-hand scale should be increased by $\sqrt{2}$ to account for the addition of the uncorrelated errors. Two examples follow: 
a) Short-term gyro specification is given as 0.5 meru. The gravity gradient is expected to be $0.1 \mathrm{sec} / \mathrm{n} . \mathrm{m}$. What is the uncompensated error growth between retros spaced $400 \mathrm{sec}$. of flying time apart, due only to the $t^{3}$ coefficient (i.e., neglecting accelerometer bias effects). The intercept is found to lie in the gyro drift region. Follow across to the right and read about 17 feet error.

b) The gyro is improved to .003 meru, and the gravity field is $0.6 \mathrm{sec} / \mathrm{n} . \mathrm{m}$. Same question. Now the intercept is in the gravity-gradient region, Follow the $0.6 \mathrm{sec} / \mathrm{n} . \mathrm{m}$. line up to the velocity line (assume $120 \mathrm{mph}$ ), and then across to the right to read about 50 feet error.

The message from the above examples is clear. For reasonably good gyros, the gravity field is likely to limit the short-term performance of the system, and it will probably pay to attempt to do some estimation of the gravity gradients being encountered. Such estimation is possible, as long as the system is not strongly into the gyro drift region, for the following reasons:

1. Gyro drift is time-correlated and gravity gradients are space-correlated. Therefore, flying in opposite directions over short periods of time it should be possible to separate the two contributors to the $t^{3}$ coefficient, by simply taking sums and differences (see Fig, 2.4-9).

2. If the system is operating well into the gravitygradient region, then any estimation of the $t^{3}$ coefficient can be entirely assigned to a gravity-gradient effect.

It has been implicitly assumed here that the updating and 


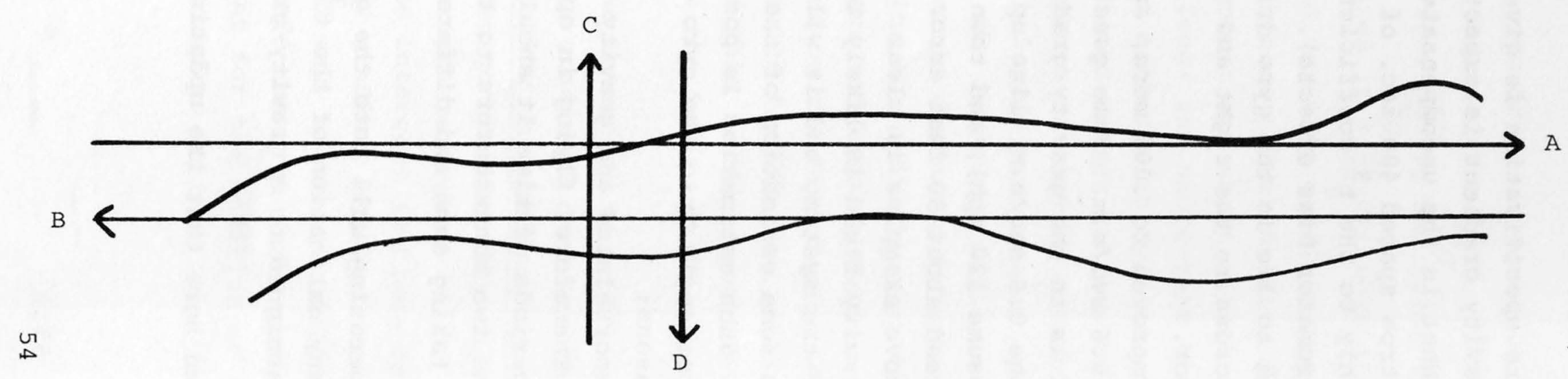

HORIZONTAL CHANNEL: $\frac{A+B}{2}=$ gyro drift component of $t^{3}$ coefficient

$\frac{A-B}{2}=$ gravity gradient component of $t^{3}$ coefficient

VERTICAL CHANNEL: $\quad \frac{A+B}{2}=$ vertical accelerometer drift component of $t^{3}$ coefficient

$\frac{A-B}{2}=$ gravity gradient component of $t^{3}$ coefficient

Fig. 2,4-9. Separation of gravity gradient and instrument errors 
estimation procedure, which uses the laser tracker, and is described elsewhere in the report, is capable of periodically estimating all the coefficients in the error curve. It is important to separate the $t^{3}$ coefficient into gravity gradient and gyro drift components, since the updated system applies these two corrections to future data in different ways. (One is time-correlated and the other is spacecorrelated.)

One of the interesting implications of this study of gravity-gradient effects is that the proposed system has the capability of creating a gravity-gradient map, in addition to the desired topographic map, at least to the extent that the retroreflector spacing provides points between which straight lines in gravity gradients can be drawn.

The vertical gravity gradient, defined as the rate of change of the magnitude of gravity (gravity anomaly) with respect to the horizontal displacement, can be treated in an analogous way to horizontal gravity gradients. The vertical gravity gradient (signified by $\frac{\partial g_{0}}{\partial x_{g}}$ ) affects the vertical channel exactly like a drifting vertical accelerometer. Using the same principles outlined above, Fig. 2,4-10 is drawn, and a comparison between the effect of accelerometer drift and vertical gravity gradients can be made. The method of region determination and read-off of uncompensated error growth is done in exactly the same way as for the previous figure, except that now the errors refer to the vertical channel. This error is not included in any of the previous vertical channel equations (neither gravity gradients nor drifting accelerometers were modeled); and therefore adds to previously considered effects, such as the effect of vertical accelerometer bias shown in Fig. 2,4-5. 


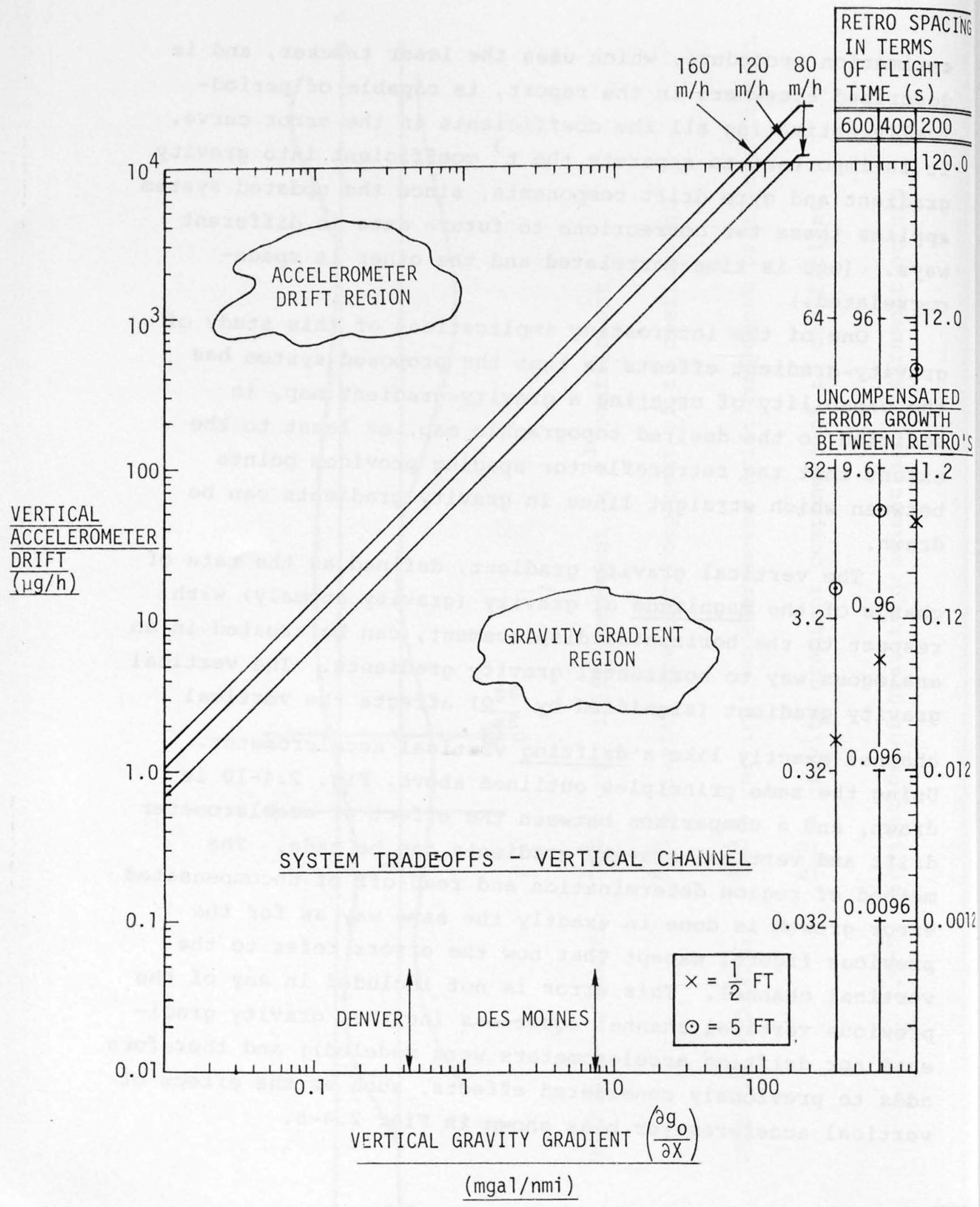

Fig. 2,4-10. Vertical error sources 


\subsubsection{Selection of Inertial Components}

The selection of inertial system components can be made based on Figures 2.4-4, 2.4-5, 2.4-6, 2.4-8 and 2.4-10. From Figure 2.4-8 it can be seen that for a severe gravity anomaly region (Des Moines), the error contributions due to gyro drift and gravimetric deflection changes with distance are about equal using commercial gyros. Consequently, difficulties arise in separating the two error sources, and a separate flight path scheme would be necessary to meet USGS requirements. This additional flight path would consist of multiple passes up and down the length of the river valley in order to separate position dependent and timedependent errors.

Using a state-of-the-art gyro, however, the errors are well within the gravity-gradient region. This permits the measurement of gravity gradients and the removal of this source of errors in terrain profiling. The measurement of gravity anomalies may also be useful for missions other than the stream-valley profiling survey. Although state-of-theart components are more expensive, it is more cost effective to design the system for their inclusion at the outset, rather than replacing second generation gyros at some future date.

The choice of accelerometer quality for the IMU can be seen in Figure 2,4-5. For a 0.5-ft. error in the vertical channel with 200 seconds between position updates, a bias error of less than $1 \mu \mathrm{g}$ is required (met by state-of-the-art integrating accelerometer). Similarly in the horizontal channels an accelerometer bias error of $10 \mu \mathrm{g}$ results in about 5.0-ft. error after 200 seconds (met by commercially available pulse-rebalanced pendulum accelerometer). Accelerometer scale-factor uncertainties can cause significant error in the vertical channel and should be less than 1 part 
per million (met by state-of-the-art integrating accelerometer). Since average acceleration is relatively low in the horizontal channels, scale factor uncertainties have little effect.

For surveys where the allowable errors are greater than $\pm 0.5 \mathrm{ft}$. and $\pm 10 \mathrm{ft}$. in the vertical and horizontal directions, the time between position updates can be increased. For example, for a $10 \mathrm{ft}$, vertical error allowance and a $1 \mu \mathrm{g}$ accelerometer bias uncertainty, time between updates can be increased to 13.3 minutes which would allow a retroreflector separation of 26.4 miles at an aircraft velocity of $175 \mathrm{ft} . / \mathrm{sec}$.

\subsubsection{Criteria for Selection of Electroöptical Components}

Components for the laser altimeter and laser tracker will be selected on the basis of achieving maximum performance in a reasonable size and weight configuration. Although the design criteria for the altimeter and tracker are similar in many respects, especially since range must be measured to the same order of accuracy, the tracker design is more critical in view of the fact that it will have an accurate two-axis gimbal configuration that will enable it to search and track over a \pm 60-degree cone. In addition, the integration of the tracker with the IMU dictates that size, weight and mechanical features be given extreme importance. Fortunately, in the tracker, the use of a cooperative target permits a smaller and lower-powered laser to be used.

Design considerations for the laser altimeter are given in Sec. 4.2. It is shown that for altitudes of approximately 3000 feet, it is easier to obtain high range accuracy using $\mathrm{CW}$ lasers rather than pulsed lasers. For diffused surfaces with a reflectivity of 0.1 , a helium-neon laser with a power slightly in excess of seven milliwatts is 
capable of providing adequate signal-to-noise ratio for accurate range measurements. Only if applications at higher altitudes are anticipated, should higher power ion lasers such as argon or krypton be considered, Pulsed lasers become attractive at greater distances, since the average power can be kept low. Ranye accuracies better than 6 inches can only be obtained with pulsed systems by using sophisticated high-frequency time-interval measurement circuitry and statistical averaging. Helium-neon lasers are known for their long life expectancy and reliability and do not require any sophisticated cooling.

Helium-cadmium or air-cooled argon lasers might be considered, if they can be found with reasonable size, weight and reliability, since they are generally available with higher power.

The laser tracker, which is necessary to update the IMU, will measure both range and angles. With a cooperative target, the required laser power is much less than that required for the laser altimeter. A CW laser source will, therefore, also be used for the tracker application, since the power required at a maximum slant range of 6000 feet is easily achieved from CW-modulated lasers. To obtain the full benefit from the range accuracy achievable, accurate pointing is necessary. As shown in Sec. 4.2, a low-powered heliumneon laser or even a CW-gallium arsenide diode have the necessary powers. The choice will be made based on the final optical-mechanical configuration that is necessary to produce a narrow beam to point at the reflector. The gallium arsenide diode is smaller, lower in weight and operates at a wavelength where silicon detectors are attractive as alternates to photomultipliers. The helium neon laser beam is easier to collimate, however, and may obtain accurate pointing with simpler optics. The use of two different wavelengths 
for the tracker and altimeter also offers the possibility of taking advantage of the two-color dispersion approach for correcting for the atmospheric index of refraction. Beam steering is most easily accomplished using a mechanical scan mode, since shaft encoders on a gimbaled assembly can provide the readout of the tracker angles. If the transmitter and receiver can be made small and lightweight, the entire assembly can be gimbaled and servoed. Alternatively, because of the size and weight, it may be found that it is more attractive to provide a mirror that can be servoed in two axes rather than the whole optical package. Different field missions might require different laser (or other sensor) choices. These might be easier to accommodate if only the mirror were servoed.

\subsubsection{Laser Safety Considerations}

For both the laser altimeter and laser tracker assume the laser spot size when reaching the ground can be as small as 6 in. This gives a $182.4 \mathrm{~cm}^{2}$ area. One milliwatt per $\mathrm{cm}^{2}$ is generally accepted as the safe level for accidental exposure to $\mathrm{CW}$ lasers emitting at wavelengths between 4000 and 7500 angstroms. Even if the laser beam were unattenuated through the atmosphere, $182.4 \mathrm{mw}$ would be safe. Since the altimeter will have a maximum power of 15-20 $\mathrm{mw}$ and the tracker laser will be even less, safety for people on the ground viewing the beam emitted from the plane does not appear to be a problem.

The safety criteria on pulsed lasers are more difficult to analyze. At high pulse-repetition rates, thermal relaxation within the retina is not permitted; therefore, the laser source may have to be treated as a CW source, In addition, biological investigations into the effects of high-repetition rate lasers are incomplete to provide injury thresholds upon 
which to base hazard criteria. Recent data indicate that with short-pulse lasers, in the nanosecond and subnanosecond regions, the safety criteria may be even more stringent than previous analysis has shown. Safety considerations are thus another reason to encourage the use of $\mathrm{CW}$ lasers for this application.

In the laboratory normal laser safety methods should be adopted. Lasers should not be left operating unattended, and whenever possible should be in shielded enclosures. The operator should avoid viewing the laser beam directly, even at low-power levels.

\subsubsection{Alignment and Calibration}

Calibration of the IMU gyros and accelerometers and the alignment of the stable platform are standardized procedures. The three accelerometers are calibrated by rotating the stable platform so that each accelerometer is positioned in turn for maximum output in the $+g(-z)$ and $-g(z)$ directions. The sum of the two readings is used to determine the accelerometer bias error $(\mu g)$, and the difference determines the accelerometer scale factor error $(\mu \mathrm{g} / \mathrm{g})$.

The stable platform is aligned to North, East and down ( $\mathrm{X}, \mathrm{Y}$ and $\mathrm{Z}$ directions, respectiveiy) by rotating about the $\mathrm{X}$ and $\mathrm{Y}$ axes to remove the gravity components along $\mathrm{Y}$ and $\mathrm{X}$, and simultaneously removing the component of earth rotation in the East ( $Y$ ) direction. This, respectively, produces local level alignment with $\mathrm{Z}$ down and aligns the $\mathrm{X}$ and $\mathrm{Y}$ axes with North and East.

Calibration of the three gyros can be accomplished by rotating the stable platform to nine different axis orientations with respect to North, East and down. The torquecommand rates for the earth-rotation rate in these positions are processed by computer to determine the misalignment 
angles, drift biases, and mass unbalance coefficients. The alignment and calibration procedures may be repeated several times as an iterative process to obtain maximum accuracy in measuring the error parameters. For a detailed development of the equations solved by the computer, see Reference 4.

\subsubsection{Subsystem Interfaces}

The system will perform only as well as the instruments and sensors that are in it - gyros, accelerometers, lasers, angle encoders, etc. The proper supporting electronics and environment must be designed into the system, however, to realize the full potential performance of these sensors.

The gyros and accelerometers in the IMU must be supplied with the proper thermal and mechanical environment. The inertial components will be stabilized at elevated temperatures, so that under all expected environmental conditions, heat will have to be applied to the thermal controllers for the system and the components. Electronics must have the necessary regulation to enable the realization of adequate sensitivities, resolution and stability as a function of time and environment.

The major interfaces are shown in Figs. 2.4-11, 2.4-12 and 2.4-13 for the IMU, tracker and altimeter. Wherever possible high-power consuming electronics will be kept external to the IMU. No refrigeration will be necessary; the system will be cooled with aircraft cabin air.

Data links between the sensors (IMU, tracker and altimeter) and the computer will be designed for serial rather than parallel communication wherever possible, minimizing the complexities associated with large cables. Resolvers will be used for transmitting the three 


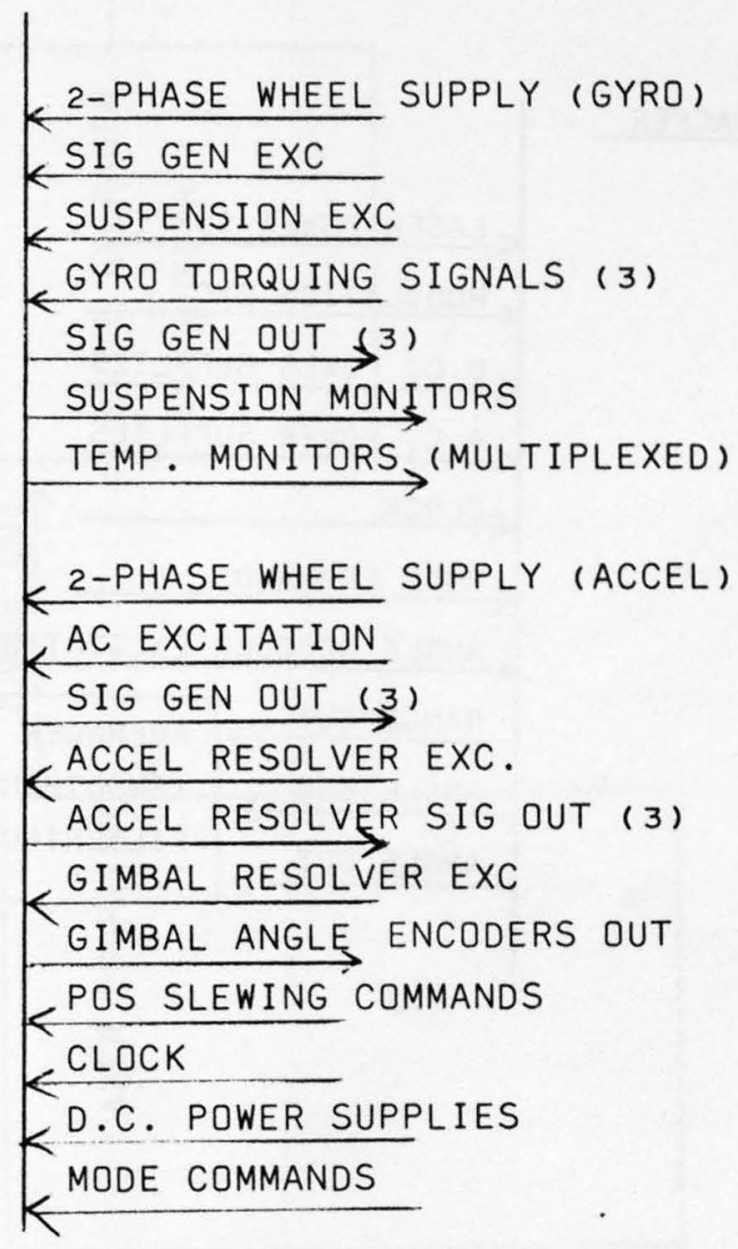

Fig. 2,4-11. Major interfaces with IMU 


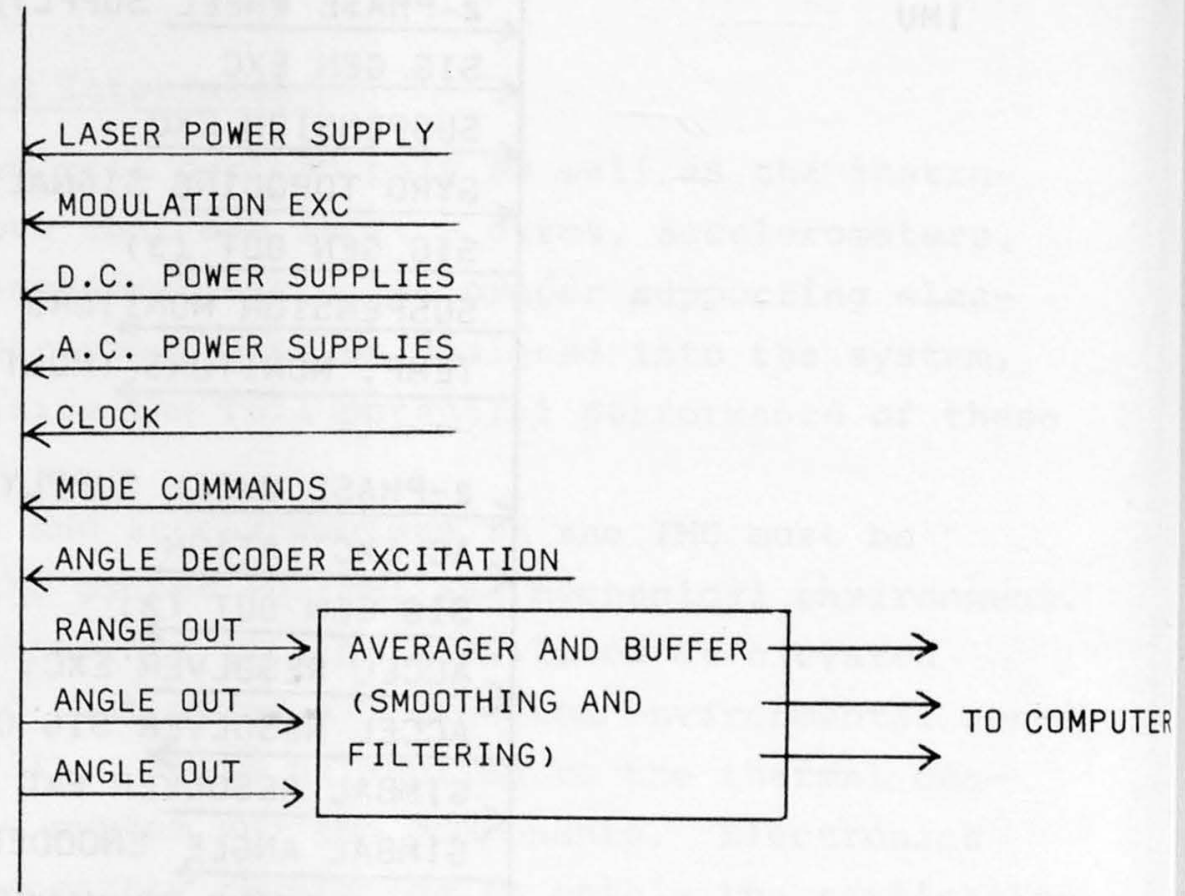

Fig. 2.4-12. Major interfaces with laser tracker 


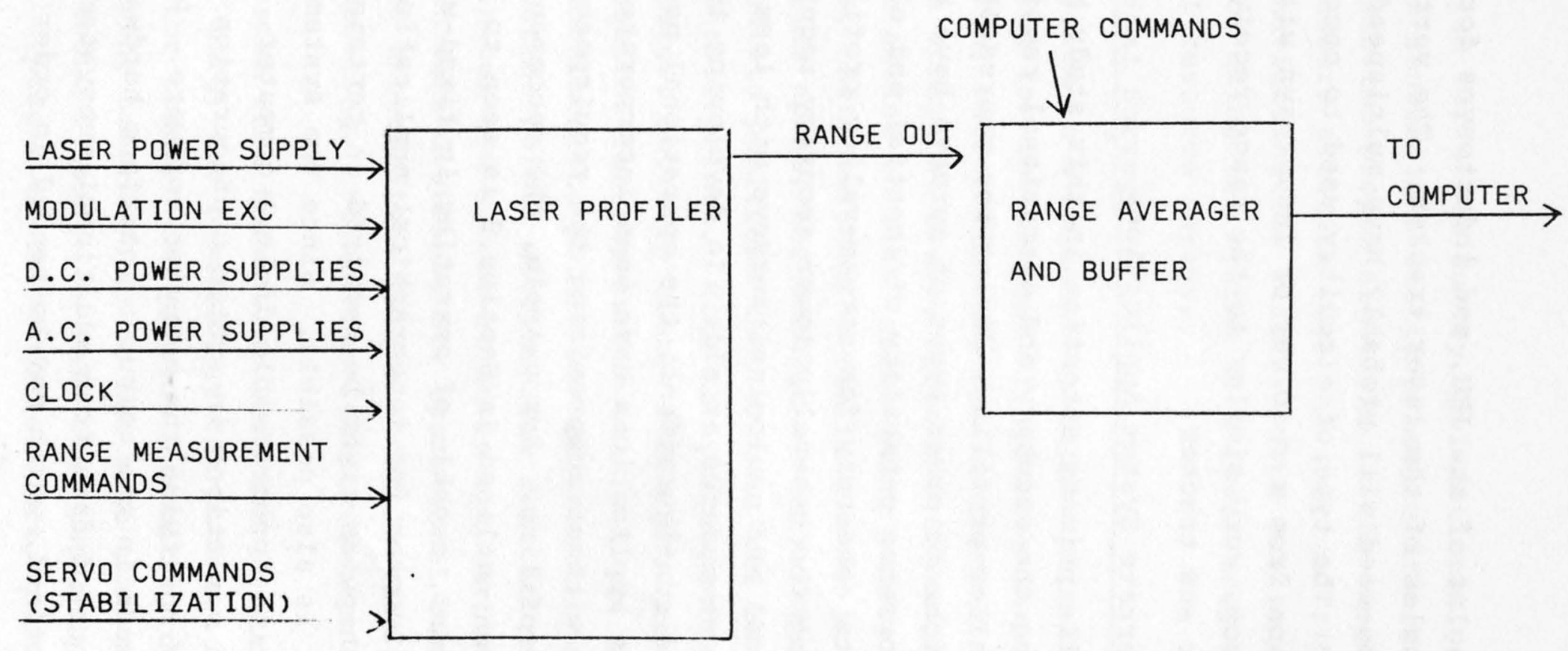

Fig. 2.4-13. Major interfaces with laser profiler 
gimbal angles of the IMU, and inductosyns for the two gimbal angles of the laser tracker. The vertical accelerometer to be used will probably have multispeed resolvers as pick-offs. The type of circuitry used to encode shaft angle information from a resolver or inductosyn will be a phaselocked loop, very similar to the range receivers used in the altimeter and tracker.

\subsection{Alternate System Applications}

While primary attention in this study has been given to meeting the accuracy and operational requirements for stream-valley profiling, during the course of the work applications to other types of surveys have become apparent. The performance capability of inertial and electroöptical components necessary for stream-valley profiling permits satisfying the generally lower accuracy requirements of topological and geological surveys with less frequent updates or no updates at all. In developing the system concepts and configurations, the operational needs of these alternate applications have been incorporated as much as possible without compromising the requirements of streamvalley profiling. For example, the recommended system configuration outlined in section 3 is seen to be vehicleindependent, capable of operating in fixed-wing aircraft or in a helicopter for topographical applications. Operation aboard ship, as might be required in certain geological surveys, is also possible. Since tbe system will incorporate a versatile, programmable digital computer, a considerable range of variations in system configuration can be accommodated by modifying the computer software. Provision can be easily made in the computer interface hardware for the acceptance of data from additional subsystems or sensors as yet undefined, which may be needed in order for the system 
to serve as a datum for other survey applications. An example of such a sensor is a static pressure transducer that, together with the proper algorithm in the computer, can introduce pressure altitude into the inertial navigation computation for navigating the aircraft from one survey site to another, or for using the system as a lower accuracy datum for natural resource surveys.

\subsubsection{Topographical Surveys}

With the system in a helicopter, rather than frequently landing or hovering for the purpose of updating the inertial position indication, retroreflectors can be tracked, thereby accomplishing the update at cruise speed and drastically reducing the time needed to link intermediate points in cadastral surveys. Retroreflectors can be placed at control points, say 20 miles apart, and additional retroreflectors placed at intermediate positions that are desired to be established as control points. With the proper software in the airborne computer, actually little different from that which would be required for the stream-valley task, the position of the intermediate points can be established with respect to the control points within a few feet in the horizontal coordinates and a foot or so in the vertical coordinate. If it were desirable for operational reasons, some or all of the intermediate points could involve stops or hovering for updating, rather than retroreflector tracking.

With the system in a fixed-wing aircraft at altitudes of 40,000 feet or more, the inertial system with a longer range retroreflector tracker can provide a position datum for an aerial-mapping camera while at the same time stabilizing the camera about 3 axes. In this case the retroreflectors would be placed up to 20 miles apart, greatly 
reducing the work of ground parties, Detailed analyses have not been made, but here again it is believed that accuracies on the order of a few feet can be readily achieved.

\subsubsection{Geological Surveys}

As set forth in section 3, the recommended system configuration separates the sensing devices, specifically the laser altimeter and camera, from the IMU-tracker subsystem that provides the positioning information. Any sensor or set of sensors that may be desired for geological survey purposes can be substituted for the laser altimetercamera. As mentioned above, full three-axis stabilization of the sensor or sensors can be provided by the reference package, if required. 
SECTION 3

RECOMMENDED SYSTEM CONFIGURATION

\subsection{System Organization}

In Section 2 the major trade-offs were given in determining an optimum configuration that would permit performing the stream-valley profiling task to the required accuracy, and at the same time be able to perform other airborne surveying and topographic tasks. It was concluded that, in order to achieve the required accuracy and perform the surveying tasks with a minimum of ground truth, an updating source, such as a laser tracker, would be required in addition to the inertial measurement unit and the laser altimeter. It was also concluded that the most accurate and cost-effective configuration would consist of tying the tracker to the stable member to provide the base motion isolation and to avoid precision angle transfers from the IMU to a physically separate tracker.

The laser altimeter with camera is kept separate because it uses a higher-powered laser than the tracker, resulting in a larger size and weight. The altimeter package needs relatively crude pointing accuracy $\left(0.1^{\circ}\right)$ compared to the tracker, and needs only be pointed vertical, Driftangle setting for the camera can be provided by the inertial system.

The recommended configuration of the IMU consists of a three-gimbaled system (pitch, azimuth and roll) containing 
three single-degree-of-freedom gyros, three accelerometers and their supporting electronics. Although the design may proceed using either high-quality commercial gyros or stateof-the-art gyros, it is more cost-effective if the design proceeds using state-of-the-art gyros at the outset, rather than with commercial gyros with plans for replacing them at some future date. In either case a state-of-the-art pendulous-integrating accelerometer would be employed in the vertical channel. In the horizontal channels, two commercially available, pulse-rebalanced pendulum accelerometers would be used in the former case and two state-of-the-art pendulous-integrating accelerometers in the latter case.

The laser tracker, which is tied to the stable member of the IMU, will consist of a transmitter (either a CW gallium arsenide laser or a low-powered neon laser), collimating and receiving optics, and a photodetection means for ranging and tracking. Search and track will be accomplished electromechanically by slewing the tracker assembly (or alternatively, a mirror) through torque motors. Precision multispeed resolvers or inductosyns will provide angle readout.

The laser altimeter, which is a separate unit, will use a helium neon laser (powered slightly over seven milliwatts) -- permitting altitude measurements up to 3000 feet above diffuse terrains with $10 \%$ reflectivity when the visual range is 5 kilometers or better -- a four- or eight-inch receiving telescope and a photodetector, The altimeter platform will have a two-axis gimbal system capable of vertical stabilization to approximately 0.1 degree.

Both the tracker and altimeter will use two or more continuous modulation frequencies. Direct detection of the photodetector output will permit heterodyning to a low intermediate frequency (IF) enabling precision phase 
measurements to be performed to determine range.

In addition to the IMU, tracker and altimeter, which are the primary sensors, the airborne system will include a digital computer, an electronics rack containing power supplies, servos and analog and digital interfaces, a control and display panel, a tape recorder and an autopilot interface.

\subsection{Cost Considerations}

On the basis of the engineering-study phase, estimates have been made of the design, fabrication, integration and testing of a prototype system. Estimates for the fabrication, integration and testing phases will be refined as the design phase progresses, since obviously decisions made during the design phase will affect the phases which are further down the line.

Estimates were made of a system using state-of-the-art gyros vs. commercially available gyros, The increase in cost for using state-of-the-art gyros is only about ten per cent of the total prototype development cost. It is not known what the cost of state-of-the-art gyros will be in the future, but it is probably safe to say that even if the costs decrease as numbers produced increase, the percentage of the total cost for a production system using state-of-the-art components will not be lower than for the prototype since non-recurring development costs do not appear in the cost of a production system. The use of state-of-the-art components, as described elsewhere in this report, besides providing greater accuracy and the ability to measure gravity gradients, allows for greater flexibility and growth potential in application to other USGS missions.

It must be borne in mind that the costs reflect a combined hardware, analysis and software effort that will result in a system which uses state-of-the-art technology in 
inertial systems, optical ranging and tracking systems as well as data processing that will find wide applicability in surveying-navigation tasks.

\section{3 Power Considerations}

The total power required for the IMU containing three gyros, three accelerometers, three gimbal servos, two tracker servos and miscellaneous electronics such as A.C. supplies, temperature controllers and analog-signal conditioners, is estimated to be under 100 watts. In addition, heater power is required to regulate the inertial components and bring the stable member up to temperature. Maximum heater power is expected to be about 40 watts.

The laser altimeter using a fairly large helium neon laser will consume a maximum of 400 watts.

The tracker using a small helium neon laser with about one milliwatt transmitter power will consume a maximum of 100 watts. If a CW gallium arsenide laser is chosen, the power consumption will be even less.

The computer, tape recorder and displays are estimated to consume approximately 500 watts.

The total power consumed by the system should, therefore, be in the neighborhood of 1000-1500 watts.

3. 4 Size and Weight Considerations

Preliminary estimates of maximum size and weight include the following:

IMU-Tracker Assembly: $1.5 \mathrm{cu}$. ft. and $451 \mathrm{bs}$. Electronics (including computer): $6 \mathrm{cu}$. ft. and 150 lbs.

Control and Display: $2 \mathrm{cu}$. ft, and $50 \mathrm{lbs}$.

Tape Recorder: $1 \mathrm{cu}$. ft. and 20 lbs. $10.5 \mathrm{cu}$. ft.

Gimbaled Laser Altimeter with Electronics: $7 \mathrm{cu}$. ft. and 150 lbs. 
It should be borne in mind that these estimates are based on experience with similar systems and that no emphasis was put in the study phase on detailed equipment design. In the design phase, efforts to minimize power consumption for thermal considerations will probably result in size and weight reduction ais well. 



\section{SECTION 4}

\section{RESULTS IN MAJOR STUDY AREAS}

\subsection{Analysis, Estimation and Simulation of Errors in Aerial Surveying}

\subsubsection{Introduction}

This section is concerned with the origin and nature of the errors associated with position determination of the airborne datum as well as those involved in tying in the position coordinates of the terrain under survey.

The character of the position error growth with time forms a very important consideration in a system where accurate determination of position is of prime importance. The knowledge of the expected pattern of the error growth not only helps estimate and thus reduce position errors, but it also helps determine the various trade-offs involved in the design of the inertial system, geometry of the devices providing ground truth, architecture of the aerial-survey path as well as the determination of operational constraints for a given environment.

The following subsections cover these topics:

- nature of errors arising in the several subsystems that determine aircraft position, such as the inertial system, laser tracker, laser altimeter

- estimation of error magnitude and growth pattern to extend position-determining capability of the airborne-inertial system 
- designing architecture of aerial-survey paths to fulfill the prescribed mission and meet surveyaccuracy requirements

- simulating the error growth and estimation that would occur during a typical aerial-survey mission to demonstrate system feasibility,

\subsubsection{Position Errors in Aerial Surveying}

\subsubsection{Introduction}

In this section we shall consider the contribution of various system elements to the error involved in determining aircraft position and the pattern of error growth. Knowledge of the growth pattern is essential in estimating errors.

Figure 4.1-1 shows essentially all the on-board and ground elements involved in aerial surveying. The platform of the IMU is maintained approximately stable in space by the three gyros, the gimbal drive servos and the computer as shown in Fig. 2.4-3 and explained in 4.1.2.3(a). Also on the platform are the accelerometers that measure acceleration in three orthogonal directions. A laser tracker complements the information from the IMU and a laser altimeter surveys the terrain profile.

The inertial system is a self-sufficient unit which indicates the aircraft position with respect to a coordinate system, e.g., latitude and longitude referenced to the 1927 NAO ellipsoid. The basic limitation of the inertial system is a slowly increasing deviation of the indicated position from the true position due to a combination of gradual platform angular drift and propagation of the computed errors arising from inaccuracies in performance of the accelerometers and gyros. The needed alternate or complementary information on the position of the aircraft is provided by the tracker, located on and referenced to the 


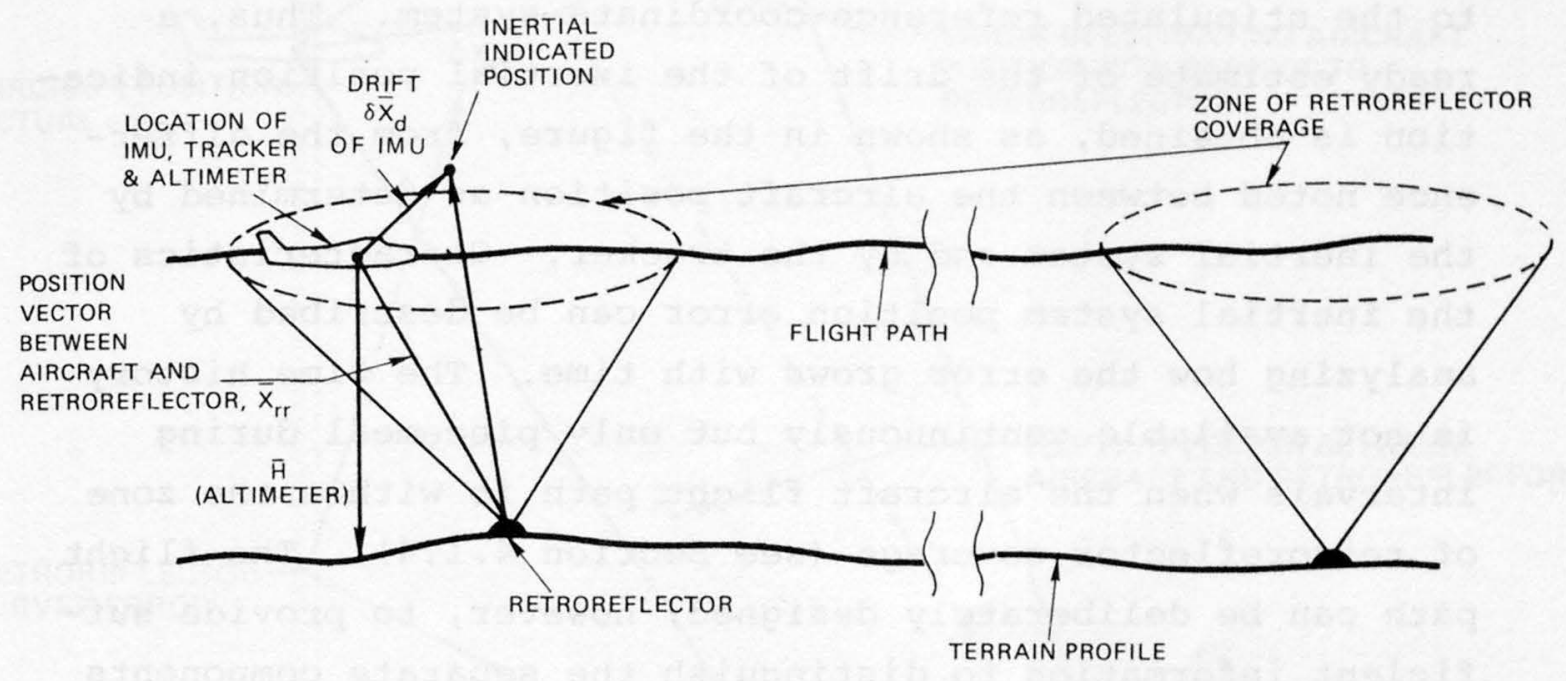

Fig, 4,1-1. Geometry of aerial profiling 
IMU. The tracker ranges on a passive retroreflector when the aircraft is flying within the zone of retroreflector coverage. These measurements of aircraft range from the retroreflector, and the angles between the range line and the on-board stabilized coordinate-reference axes, together with knowledge of the actual retroreflector position, are sufficient ${ }^{*}$ to determine the aircraft position, with respect to the stipulated reference-coordinate system. Thus, a ready estimate of the drift of the inertial position indication is obtained, as shown in the figure, from the difference noted between the aircraft position as determined by the inertial system and by the tracker. Characteristics of the inertial system position error can be described by analyzing how the error grows with time. The time history is not available continuously but only piecemeal during intervals when the aircraft flight path is within the zone of retroreflector coverage (see Section 4.1.4). The flight path can be deliberately designed, however, to provide sufficient information to distinguish the separate components of the error. Thus, by distinguishing these components of the error while the tracker is within the zone of retroreflector coverage, predictions of the error growth are possible when the aircraft is outside the zone.

\subsubsection{Error Sources in Determining Aircraft Position}

In this section we consider the relationship between the errors in the two alternate but complementary methods of determining aircraft position viz, the inertial system and the tracker in conjunction with a retroreflector. Figure 4.1-2 shows the measurement geometry of the pertinent errors

IMU angular misalignments, though small, must be estimated and taken into account, as described in Appendices $C$ and D. 


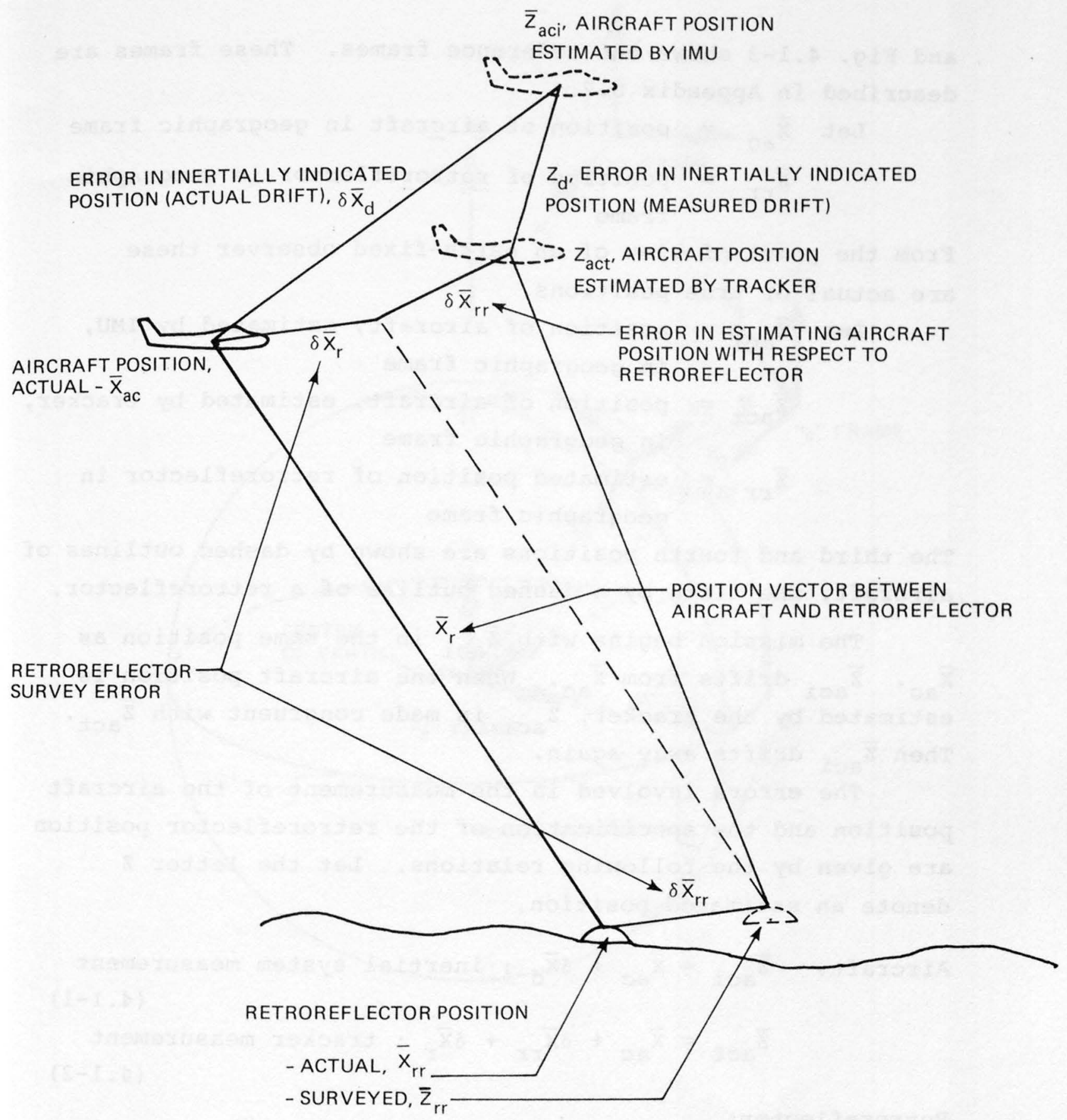

Fig. 4.1-2. Geometry of Fig. 4.1-1 with addition of retroreflector survey error, $\delta \bar{x}_{r r}$, and error in retroreflector position, $\delta \bar{x}_{r}$ 
and Fig. 4.1-3 shows the reference frames. These frames are described in Appendix D.2.

Let $\overline{\mathrm{x}}_{\mathrm{ac}}=$ position of aircraft in geographic frame

$$
\overline{\mathrm{x}}_{\mathrm{rr}}=\text { position of retroreflector in geographic }
$$
frame

From the point of view of an earth-fixed observer these are actual or true positions.

Let $\overline{\mathrm{z}}_{\mathrm{aci}}=$ position of aircraft, estimated by IMU, in geographic frame

$$
\begin{aligned}
\overline{\mathrm{z}}_{\text {act }}= & \text { position of aircraft, estimated by tracker, } \\
& \text { in geographic frame } \\
\overline{\mathrm{z}}_{\mathrm{rr}}= & \text { estimated position of retroreflector in } \\
& \text { geographic frame }
\end{aligned}
$$

The third and fourth positions are shown by dashed outlines of aircraft, the fifth by a dashed outline of a retroreflector.

The mission begins with $\bar{z}_{\text {aci }}$ in the same position as $\overline{\mathrm{x}}_{\mathrm{ac}} \cdot \overline{\mathrm{Z}}_{\mathrm{aci}}$ drifts from $\overline{\mathrm{x}}_{\mathrm{ac}}$. When the aircraft position is estimated by the tracker, $\overline{\mathrm{Z}}_{\text {aci }}$ is made congruent with $\overline{\mathrm{Z}}$ act Then $\overline{\mathrm{Z}}_{\text {aci }}$ drifts away again.

The errors involved in the measurement of the aircraft position and the specification of the retroreflector position are given by the following relations. Let the letter $\mathrm{Z}$ denote an estimated position.

Aircraft: $\quad \overline{\mathrm{Z}}_{\mathrm{aci}}=\overline{\mathrm{x}}_{\mathrm{ac}}+\delta \overline{\mathrm{x}}_{\mathrm{d}}$; inertial system measurement

$$
\overline{\mathrm{z}}_{\text {act }}=\overline{\mathrm{x}}_{\mathrm{ac}}+\delta \overline{\mathrm{x}}_{r r}+\delta \overline{\mathrm{x}}_{r} ; \text { tracker measurement }
$$

Retroreflector:

$$
\overline{\mathrm{z}}_{r r}=\overline{\mathrm{x}}_{r r}+\delta \overline{\mathrm{x}}_{r r} ; \text { "survey" measurement }(4.1-3)
$$




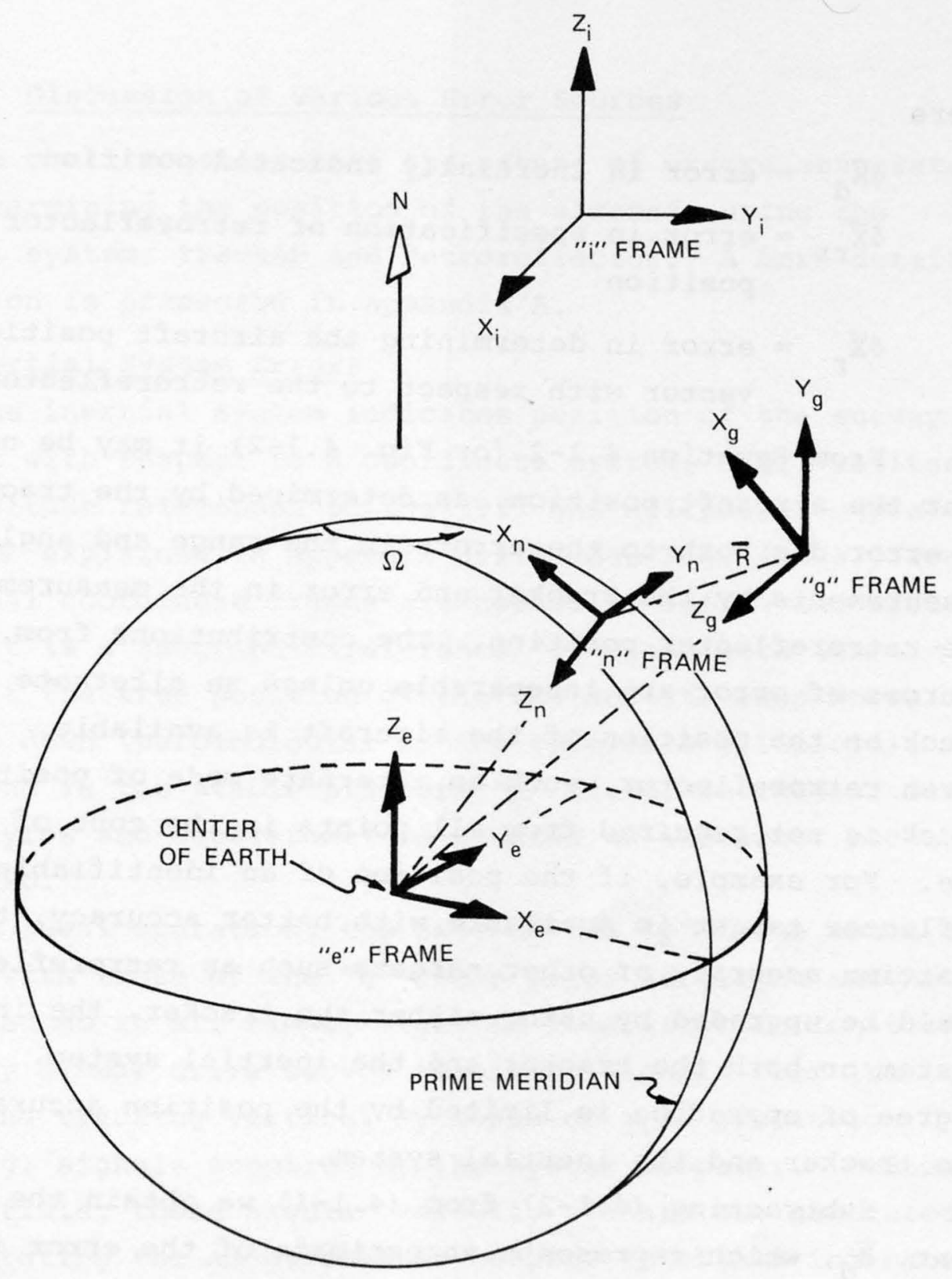

Fig. 4.1-3. Reference frames 
where

$$
\begin{aligned}
\delta \overline{\mathrm{x}}_{\mathrm{d}}= & \text { error in inertially indicated position } \\
\delta \overline{\mathrm{x}}_{r r}= & \text { error in specification of retroreflector } \\
& \text { position } \\
\delta \overline{\mathrm{x}}_{r}= & \text { error in determining the aircraft position } \\
& \text { vector with respect to the retroreflector }
\end{aligned}
$$

From Equation 4.1-2 (or Fig. 4.1-2) it may be noted that the aircraft position, as determined by the tracker, is in error due both to the errors in the range and angle measurements by the tracker and error in the measurement of the retroreflector position. The contributions from the two sources of error are inseparable unless an alternate mode of check on the position of the aircraft is available. For a given retroreflector, such an alternate mode of position check is not required from all points in the zone of coverage. For example, if the position of an identifiable reflector target is available with better accuracy, the position accuracy of other targets such as retroreflectors could be upgraded by using either the tracker, the inertial system or both the tracker and the inertial system. The degree of upgrading is limited by the position accuracy of the tracker and the inertial system.

Subtracting (4.1-2) from (4.1-1) we obtain the measurement, $\bar{z}_{d}$, which represents an estimate of the error in the inertially indicated position, $\delta \bar{x}_{d}$.

$$
\overline{\mathrm{z}}_{\mathrm{d}}=\delta \overline{\mathrm{x}}_{\mathrm{d}}-\delta \overline{\mathrm{x}}_{r}-\delta \overline{\mathrm{x}}_{r r}
$$

where

$$
\overline{\mathrm{z}}_{\mathrm{d}}=\overline{\mathrm{z}}_{\mathrm{aci}}-\overline{\mathrm{z}}_{\mathrm{act}}
$$




\subsubsection{Discussion of Various Error Sources}

We shall consider here the nature of errors associated with determining the position of the aircraft using the inertial system, tracker and retroreflector. A more detailed discussion is presented in Appendix A.

\section{(a) Inertial System Errors}

The inertial system indicates position of the survey aircraft with respect to a coordinate system, e.g., latitude and longitude referenced to the 1927 NAO ellipsoid. (The "e" frame is explained in Appendix D.2.) See Fig. 4.1-3. Two additional coordinate frames are needed to define the system: The first is a geographic reference "g" frame with its origin at the true position of the IMU and its axes North, East and down (perpendicular to the reference ellipsoid). The second is the stable platform " $p$ " frame, defined or fixed by the gyros and accelerometers mounted on the inner package of the IMU.

In ideal operation, the axes of the "p" frame would be aligned with those of the "g" frame prior to flight and be maintained so at all times, isolated from aircraft rotational motion by gimbal drive servos receiving signals from the gyros, and tracking vertical by means of gyro precession (torquing) signals supplied by the system computer. Reduced to essentials, these angular velocity signals are generated by integrating the accelerometer outputs to obtain indicated ground speed while dividing by the earth's radius.

The errors in the inertial position indication arise primarily due to inaccuracies in gyro and accelerometer performance. Inertial system error dynamics (referred to "p" frame) are simply given by:

$$
\delta \ddot{\bar{x}}_{p}=\delta \bar{A}+\delta \bar{g}
$$


where

$$
\begin{aligned}
& \delta \ddot{\bar{x}}_{\mathrm{p}}=\begin{array}{l}
\text { the position error in the inertial } \\
\text { system output }
\end{array} \\
& \delta \overline{\mathrm{A}}= \text { the error in the accelerometer output } \\
& \text { and } \delta \bar{g}= \text { the error in the gravitational accelera- } \\
& \text { tion mechanized in the computer and IMU } \\
& \delta \dot{x}_{\mathrm{p}}(0)= \text { unknown } \\
& \delta \mathrm{x}_{\mathrm{p}}(0)=\text { unknown }
\end{aligned}
$$

The errors in the apparent acceleration components sensed by the accelerometers arise primarily from the zerooutput error (known as bias error also) and the scale-factor error. Due to the angular deviation of the stable platform with respect to the "g" reference frame defined above, caused by gyro drift and inaccuracies involved in precessing the gyros, besides sensing the acceleration along the specified inertial axis, the accelerometers also sense part of the acceleration components along the other two axes of the triad.

In summary, the error $\delta \overline{\mathrm{A}}$ can be expressed mathematically as follows with

$$
\delta \bar{A}=\bar{b}+k_{a} \bar{A}-\bar{\psi} \times \bar{A}
$$

where

$$
\begin{aligned}
\bar{b}= & \text { bias error } \\
\mathrm{k}_{\mathrm{a}} \overline{\mathrm{A}}= & \text { scale factor error } \\
\bar{\psi}= & \text { small angle deviation of the "p" frame } \\
& \text { measured with respect to airborne } \\
& \text { geographic "g" frame } \\
\bar{A}= & \text { apparent acceleration of the vehicle in } \\
& \text { the "p" frame }
\end{aligned}
$$

Now let us consider the second term in the right-hand side of Equation (4.1-6). The gravitational acceleration is 
a function of $\bar{x}_{g}$, the true position of the vehicle in the geographic frame. Thus, the error, $\delta \vec{g}$, in the gravitational acceleration, consists of two terms with

$$
\delta \bar{g}=\delta \bar{g}\left(\delta \bar{x}_{p}\right)+\delta \bar{g}\left(\bar{x}_{g}\right)
$$

The first term on the right-hand side of (4.1-6) arises due to the fact that the gravity model supplies the gravitational acceleration for a position which is indicated by the inertial system and is, therefore, in error by $\delta \bar{x}_{p}$. Thus, in a sense, it is a feedback term in the IMU error equation.

It provides a stabilizing feedback in the horizontal channels leading to Schuler oscillations with a period of 84 minutes, and a destabilizing feedback leading to exponential error growth in the vertical channel. The feedback is relatively slow in taking effect, and thus its influence on the error growth in all three channels is hardly noticeable for times less than one tenth of the Schuler period. Since the time between position fixes for the IMU is expected to be substantially less than 8 minutes, the effect of the gravity feedback term $\delta \bar{g}\left(\delta \bar{x}_{g}\right)$ can be neglected.

The second term on the right-hand side of Equation $(4,1-8)$ arises due to errors in modeling the actual gravitational acceleration obtaining at different parțs of the terrain that is being surveyed. Unless the results of a gravimetric survey of the terrain are available, the gravityvector deviations from the gravity model would constitute a part of the operating environment of which one has no a priori knowledge. The deleterious effect of gravity-vector deviations can be contained within acceptable limits with the help of occasional position fixes from the tracker during the aerial-survey mission. 
(b) Effect of Instrument and Gravity Model Errors

Among the slow and fast (low and high frequency) components of the instrument and gravity model errors, the slow (bias) component of the error becomes more of a concern because of its impact on the position-error growth.

Considering a component of the error equation, we get

$$
\delta \ddot{x}_{p}=b
$$

where b represents the corresponding component of the sum of the instrument and gravity-model error vectors. The error growth in $\delta \mathrm{x}$ due to the slow and fast components of $\mathrm{b}$ is given by the following equations:

$$
\delta \mathrm{x}_{\mathrm{p}}=\frac{1}{2} \mathrm{~b} \mathrm{t}^{2} \text { for } \mathrm{a} \text { bias component }
$$

and

$$
\sigma_{\delta x_{p}}=\frac{1}{\sqrt{3}} \sigma_{b} t^{3 / 2}
$$

for a fast (high frequency) random component with zero mean and variance $\sigma_{b}$. The mean of $\delta x$ is zero for the fast (high frequency) component of the noise, the deviations from the mean being specified by the variance $\sigma_{\delta x_{p}}$.

to $t^{2}$ for a bias component and $t^{3 / 2}$ for a high-frequency component. Thus, for example,

$$
\frac{{ }^{\sigma} \delta x_{p}}{\delta x_{p}} \simeq \frac{1}{10}
$$

$$
\begin{array}{ll}
\text { for } & t=100 \text { secs. } \\
\text { and } & \sigma_{b}=b .
\end{array}
$$


In other words, for the same error growth, we can stand a high-frequency component with variance 10 times larger than the mean component of the noise.

(c) Range Error of The Tracker System

There will be some range error due to the following effects. Referring to Fig, 4.1-2, the tracker basically determines the magnitude (range) and the orientation of the position vector $\overline{\mathrm{x}}_{r}$ between the aircraft and the retroreflector. The position of the aircraft is determined by erecting this vector $\bar{x}_{r}$ from the position of the retroreflector. On board the aircraft the orientation of $\bar{x}_{r}$ can only be measured with reference to the stable platform (p) frame. Errors arise in determining the range and orientation of $\bar{x}_{r}$ with respect to the earth-fixed reference frame. The range, $r$, which is the magnitude of $\bar{x}_{r}$, is determined from $t$, the elapsed time between the transmitted light signal and the signal reflected from the retroreflector. Thus,

$$
r=\frac{1}{2} v t
$$

where $\mathrm{v}$ is the velocity of light in air.

For the sake of simplicity in discussion, we have assumed the velocity of the light beam from the tracker to be constant during the entire time of its travel. A discussion on the effect of non-uniformity in the velocity of light is given in section 4.2,1.2.1.

The velocity of light in air is related to the velocity of light in vacuum, $\mathrm{c}$, by the index of refraction $\mathrm{n}$ with

$$
\mathrm{v}=\frac{\mathrm{c}}{\mathrm{n}}
$$


The index of refraction $\mathrm{n}$ is given by

$$
n=f\left(T, P, P_{W V}, \lambda\right)
$$

where

$T, P, P_{W V}$ and $\lambda$, respectively, are the air temperature, pressure, water vapor pressure and the wavelength of the light signal.

The error $\delta r$, in determining the range of the aircraft from the retroreflector, can be obtained by differentiating Equation 4.1-9:

$$
\delta r=\left(\frac{\delta t}{t}-\frac{\delta n}{n}\right)
$$

where

$$
\delta n=\frac{\partial f}{\partial T} \delta T+\frac{\partial f}{\partial P} \delta P+\frac{\partial f}{\partial P}{ }_{W V} \delta P{ }_{W V}
$$

Range error, $\delta r$, occurs when pointing in general direction of retroreflector.

The error is due both to measuring the elapsed time and to the modeling of the atmospheric refractive index. Section 4.2.1.2.1 discusses in detail these errors and concludes that their net effect on range measurement errors is well within $0.1 \mathrm{ft}$. at a range of $6000 \mathrm{ft}$. The effect of atmospheric turbulence on the laser-tracker pointing accuracy is discussed in Appendix B.

Errors in measuring the orientation of the position vector $\bar{x}_{r}$ with respect to the reference coordinate system arise due to two reasons:

1) errors in determining the orientation of the position vector $\bar{x}_{r}$ with respect to the stable platform (p) frame; and

2) errors in the knowledge of the orientation of the 
stable platform "p" coordinate frame with respect to the geographic reference " $g$ " coordinate frame.

The error in determining the components of $\overline{\mathrm{x}}_{r}$ with respect to the reference frame is given by $\bar{x}_{r} x \bar{\psi}_{t}$, the vector cross product of the position vector $\overline{\mathrm{x}}_{r}$ and the orientation error vector $\bar{\psi}_{t}$.

Thus, the resulting error $d \bar{x}_{r}$ in determining $\bar{x}_{r}$ is given by

$$
\delta \overline{\mathrm{x}}_{r}=\delta R \frac{\overline{\mathrm{x}}_{r}}{r}+\overline{\mathrm{x}}_{r} \times \bar{\psi}_{t}
$$

where $\delta R$ is from Eq. $(4.1-12)$ and $\widetilde{x}_{r} / R$ represents the unit vector in the direction of $\overline{\mathrm{x}}_{\mathrm{r}}$.

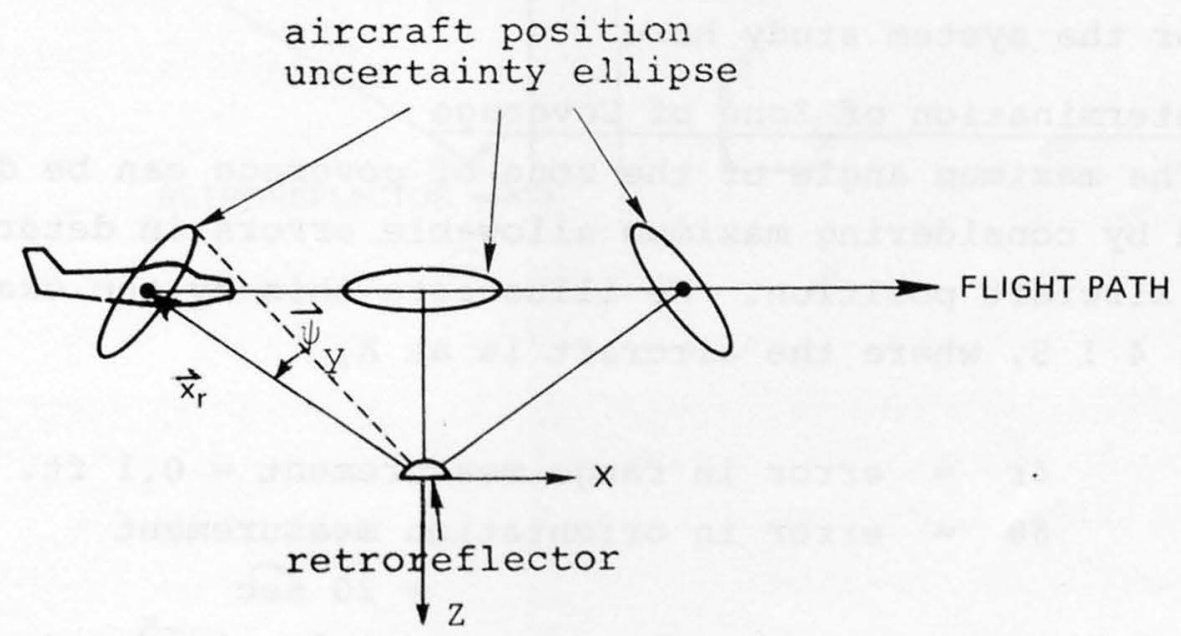

Fig 4,1-4, Effect of range and angle measurement errors 
The range measurement primarily removes the uncertainty in the location of the aircraft in the direction of the vector $\overline{\mathrm{x}}_{\mathrm{r}}$ and not in the direction perpendicular to it. In the case of the orientation-angle measurements, the reverse is true. The effect of the two errors is thus complementary in nature, as further explained in Figure 4,1-4. The figure shows the uncertainty ellipse in determining the position of the aircraft from the range and angle measurements. The uncertainty, shown to be less along the direction of $\bar{x}_{r}$ vector and more along the perpendicular direction, reflects the fact that the range measurements are considerably more accurate than the angle measurements. Provided the IMU misalignment angles are small, the accuracy achievable in the angle measurements is primarily limited by the allowable size and weight of the angle-readout transducers. The error values of $0.1 \mathrm{ft}$. for range error and 20 arcsec. for the orientation error are used for the system study here.

(d) Determination of Zone of Coverage

The maximum angle of the zone of coverage can be delimited by considering maximum allowable errors in determining aircraft position. We illustrate this by the example of Fig. 415 , where the aircraft is at $A$,

$$
\begin{aligned}
\delta r= & \text { error in range measurement }=0.1 \mathrm{ft} . \\
\delta \theta=\text { error in orientation measurement } & =20 \mathrm{sec} \\
& =9.7 \times 10^{-5} \mathrm{rad} . \\
h=3000 \mathrm{ft} . &
\end{aligned}
$$

The horizontal and vertical components, $\delta x$ and $\delta z$, of the position error are

$$
\begin{aligned}
& \delta \mathrm{x}=\mathrm{h} \delta \theta+\delta r \sin \theta \\
& \delta \mathrm{z}=\delta r \cos \theta+h \tan \theta \delta \theta
\end{aligned}
$$




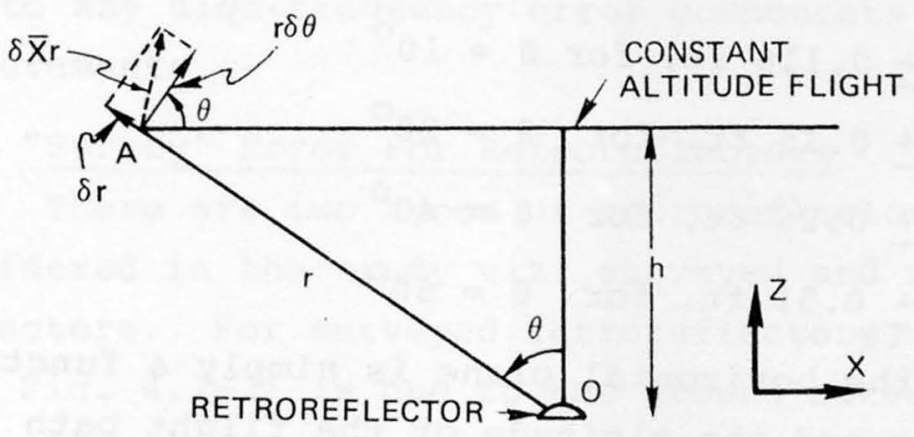

Fig. 4.1-5. Position error, $\delta \bar{x}_{\text {, }}$, of aircraft at $A$ composed of range error, $\delta r$, and orientation error, $r d \theta$ 
Since $\delta \theta$ and $\delta r$ are uncorrelated errors, the rms errors for $\delta x$ and $\delta z$ are given by

$$
\begin{aligned}
\sigma_{\delta x} & =\sqrt{(h \delta \theta)^{2}+(\delta r \sin \theta)^{2}} \\
\text { and } \delta_{\delta z} & =\sqrt{\left(\delta r \cos \theta i^{2}+(h \tan \theta \delta \theta)^{2}\right.}
\end{aligned}
$$

Substituting the above values of $\delta r, \delta \theta$ and $h$,

$$
\sigma_{\delta x} \simeq h \delta \theta \simeq \pm 0.3 \mathrm{ft} .
$$

and

$$
\begin{aligned}
\sigma_{\delta z} & =\sqrt{0.1^{2}+(0.3 \tan \theta)^{2}} \\
& = \pm 0.116 \mathrm{ft} . \text { for } \theta=10^{\circ} \\
& = \pm 0.15 \mathrm{ft} . \text { for } \theta=20^{\circ} \\
& = \pm 0.26 \mathrm{ft} . \text { for } \theta=40^{\circ} \\
& = \pm 0.51 \mathrm{ft} . \text { for } \theta=60^{\circ}
\end{aligned}
$$

The error in the horizontal plane is simply a function of the angular error and the altitude of the flight path and, thus for a given $\delta \theta$, its magnitude remains nearly constant irrespective of the angle $\theta$ of the line of sight with respect to the vertical.

Thus, for a given accuracy requirement for the updates of $\mathrm{x}$ and $\mathrm{y}$ components of the aircraft position in the horizontal direction, the primary limiting factor is the maximum angular error expected in determining the orientation of the linerof-sight vector.

At large angles of incidence, the increase in the angular errors due to increased beam-spot size and beam bending due to atmospheric refraction effects become more important, and thus form the limiting considerations in determining the maximum angle of the cone coverage from the point 
of view of required accuracy in the update of the horizontal position of the aircraft.

On the other hand, the increasingly larger vertical component of the angular component error $(0.3 \mathrm{ft}$.$) at the$ higher angles of incidence contributes to larger errors in the estimate of the vertical component of the aircraft position. The maximum allowable angle of incidence thus depends upon the required accuracy for the vertical update. For example, $\theta_{\max }=20^{\circ}$ for a vertical accuracy requirement of $0.15 \mathrm{ft}$.

It may be finally commented that we have not assumed any filtering or processing which would reduce the errors due to any high-frequency error components in the position measurements.

(e) "Survey" Error For Retroreflectors

There are two types of retroreflectors that have been considered in the study viz. surveyed and unsurveyed retroreflectors. For surveyed retroreflectors, the error $\delta \bar{x}_{r r}$ (see Fig. 4,1-2) is due to the ground survey error. We shall primarily consider the error in determining the coordinates of the unsurveyed or walked-in retroreflectors by means of an aerial survey during the mission. The coordinates are needed with sufficient accuracy that the unsurveyed retroreflectors can be used during the aerial-profiling phase of the mission to supply the position fixing (ground truth) information to contain the errors in inertially indicated position within acceptable limits.

Fig. 4.1-6 shows the measurement geometry for determining the position of the unsurveyed retroreflector by determining its range and orientation from the aircraft by means of the tracker. The sum of the errors in the range and orientation measurement and in the knowledge of the position of the aircraft constitutes the error in the 


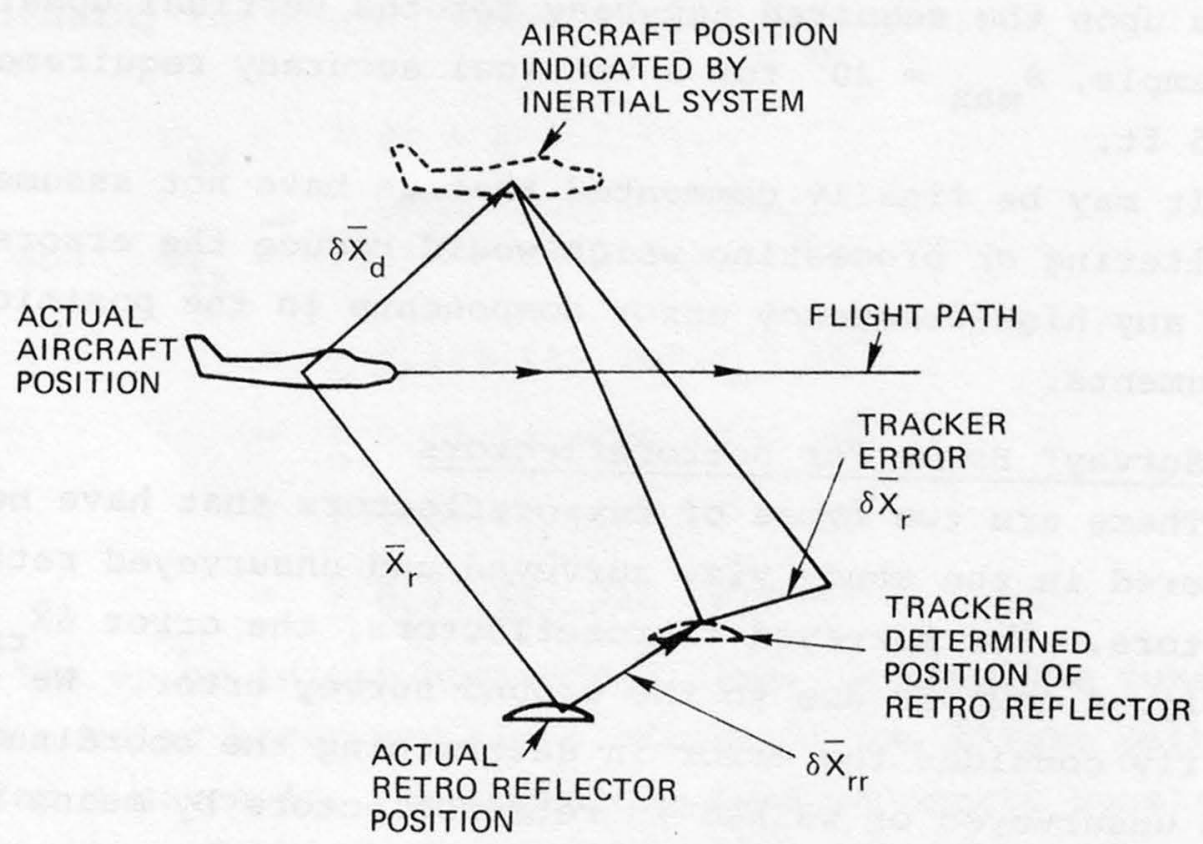

Fig. 4.1-6. Determination of position of unsurveyed retroreflectors 
determination of the position of the retroreflector.

Let $\overline{\mathrm{x}}_{\text {retro }}=$ the position of the retroreflector with respect to the earth-fixed reference frame and Thus,

$\overline{\mathrm{z}}_{\text {retro }}=$ the estimated position of the retroreflector.

$$
\overline{\mathrm{z}}_{\text {retro }}=\overline{\mathrm{x}}_{\text {retro }}+\delta \overline{\mathrm{x}}_{\text {rr }}
$$

where $\delta \bar{x}_{r r}$, the "survey" error is given by

$$
\delta \overline{\mathrm{x}}_{r r}=\delta \overline{\mathrm{x}}_{\mathrm{d}}+\delta \overline{\mathrm{x}}_{r}
$$

The error in the estimate of $\bar{x}_{\text {retro }}$ could be further reduced by continuously tracking the retroreflector during the flight of the aircraft through the zone of coverage and suitably processing the resulting measurements $\bar{z}_{\text {retro }}(t)$, to obtain a better estimate of the position of the retroreflector.

It may be remarked here that the accuracy requirements for the knowledge of the position of the retroreflector has several implications for the system design. The expected magnitude of $\delta \bar{x}_{d}$, the error in inertially indicated position, is determined by the errors in the estimate of the patterns in the inertial system-error growth resulting due to the biases in the instrument and the gravity-model errors. The errors in the estimate of the patterns in the inertial-system error growth depend upon the accuracy of the IMU whose specifications are desired as a part of the system design. The error $\delta \overline{\mathrm{X}}_{\mathrm{d}}$ has another component which depends upon another factor, viz, the gravity-model errors, determined by the geological features of the terrain under survey, over which the system designer has no control. The magnitude of the inertial-system error (unestimated) also depends upon the pattern of the flight path and the time elapsed since the last position fix. Thus the impact of a given gravity environment (also IMU accuracy specifications) on the expected position errors can be limited by a proper design 
of the flight patterns and by specifying the maximum allowable time between flight updates as set forth in the appendices.

\subsubsection{Estimation of Errors and Data Processing}

\subsubsection{Estimation of Errors}

Table 4.1-1 lists significant sources of error that arise in the different ways of determining position, the symbols used, where defined, where illustrated and where discussed. We have considered the errors arising in determining position by means of tracker, retroreflector and inertial system. The errors in determining the vertical clearance of the aircraft from the terrain by means of a laser profiler are of the same kind as encountered in the tracker except that the effect of range-vector orientation errors are secondary to the effect of range errors. The errors in determining the position of the airborne datum from which the survey is carried out are of prime interest in aerialsurvey missions.

The need for estimation of an individual error is governed by the frequency spectrum of the error and its relative impact on the determination of the airborne datum. The bias type of errors which change very slowly with time are at the low end of the frequency spectrum and the fastvarying errors are at the high end of the frequency spectrum. The estimation techniques and their complexity depend on the frequency spectrum of the error.

The high-frequency errors could be handled relatively easily by taking a large sample of measurements and performing an appropriate averaging of the measurements. Since the high-frequency errors do not have any steady component, the effect of high-frequency errors is considerably reduced by the time averaging of the measurement, The process of 


\begin{tabular}{|c|c|c|c|c|c|}
\hline Instrument & Errors & Symbol & $\begin{array}{l}\text { Where Defined } \\
\text { (Section No.) }\end{array}$ & $\begin{array}{l}\text { Where Illustrated } \\
\text { (Figure No.) }\end{array}$ & $\begin{array}{l}\text { Where Discussed } \\
\text { (Section No.) }\end{array}$ \\
\hline Tracker & $\begin{array}{l}\text { Range Error } \\
\text { - timing error } \\
\text { - atmospheric refraction modeling errors } \\
\text { Range Vector Orientation Error } \\
\text { - angle readout error } \\
\text { - IMU coordinate system drift } \\
\text { - beam bending due to refraction effects } \\
\text { - beam spot size errors } \\
\text { - tracker servo errors }\end{array}$ & $\begin{array}{l}\delta r \\
\cdots \\
\delta \bar{x}_{r} \\
\delta \theta \\
\delta \overline{\mathrm{x}}_{\mathrm{d}} \\
\cdots\end{array}$ & $\begin{array}{l}4.1 .2 .3(c) \\
-. \\
4.1 .2 .2 \\
4.1 .2 .3(d) \\
4.1 .2 .2 \\
4.1 .2 .3(c)\end{array}$ & $\begin{array}{c}4.1-5 \\
-- \\
4.1-2 \\
4.1-4 \\
4,1-2 \\
--\end{array}$ & $\begin{array}{l}4 \cdot 1 \cdot 2 \cdot 3(\mathrm{c}) \\
\text { App. B } \\
4 \cdot 1 \cdot 2 \cdot 3(\mathrm{c}) \\
4 \cdot 1 \cdot 2 \cdot 3(\mathrm{~d}) \\
4 \cdot 1 \cdot 2 \cdot 2 \\
\text { App. B }\end{array}$ \\
\hline Retroreflector & Survey Error & $\delta \bar{x}_{r r}$ & $4.1 .2,3(e)$ & $4 \cdot 1-2$ & $4 \cdot 1 \cdot 2 \cdot 3(\mathrm{e})$ \\
\hline IMU & $\begin{array}{l}\text { Accelerometer Errors } \\
\text { - bias errors } \\
\text { - scale factor errors } \\
\text { Gyro Errors } \\
\text { - drift rates } \\
\text { - mass unbalance errors } \\
\text { - scale factor torquing errors } \\
\text { Platform misalignment angles } \\
\text { Gravity Model Errors } \\
\text { - gravity anomaly and vector deflection } \\
\text { - change of anomaly and deflection } \\
\text { gradient with distance }\end{array}$ & $\begin{array}{l}\overline{\mathrm{B}}_{\mathrm{a}} \\
\overline{\mathrm{K}}_{\mathrm{a}} \\
\bar{\varepsilon} \\
\stackrel{\star}{\mathrm{U}} \\
\stackrel{\mathrm{K}}{\mathrm{T}}^{\psi} \\
\delta \bar{g} \\
\delta \mathrm{g}_{0} \\
\frac{\partial \delta \mathrm{g}_{0}}{\partial \overline{\mathrm{x}}}\end{array}$ & $\begin{array}{l}2.4,2.1 \\
4,1,2 \cdot 3(\mathrm{a}) \\
2.4 .2 .1 \\
\text { Fig. E-2 } \\
\text { Fig. E-2 } \\
2.4 .2 .1 \\
4.1 .2 .3(\mathrm{a}) \\
\text { App. A.11 } \\
\text { App. A.11 }\end{array}$ & $\begin{array}{l}-- \\
-- \\
-- \\
-- \\
-- \\
-- \\
-- \\
-- \\
\text { Figs, } A-3 \\
\text { and } \mathrm{A}-4\end{array}$ & $\begin{array}{l}4.1 .2 .3(a) \\
\text { App. E.1 } \\
\text { App. E.1 } \\
\text { App. E.1 } \\
\text { App. E.1 } \\
\text { App. A. } 3 \\
\text { App. A.11 } \\
\text { App. A.11 } \\
\text { App. A.11 }\end{array}$ \\
\hline Altimeter & $\begin{array}{l}\text { Height Error } \\
\text { - timing error } \\
\text { - atmospheric refraction modeling errors } \\
\text { - uncertainties due to obstructions, } \\
\text { e.g., foliage or roughness of terrain }\end{array}$ & -- & $\begin{array}{l}4.1 .3 \cdot 1 \\
4.1 .3 \cdot 1 \\
2.3 .2\end{array}$ & $\begin{array}{c}-- \\
-. \\
2.3-2\end{array}$ & $\begin{array}{c}-- \\
-- \\
2.3 .2\end{array}$ \\
\hline
\end{tabular}

Table 4.1-1. Significant Sources of Error 
averaging would have to appropriately weigh the measurement to reflect the information content and the noise content in each measurement by employing weighted least square or Kalman filter schemes (see Appendix C).

(a) Estimation of Inertial System Error Growth Pattern

The separate effects of bias, scale factor and platform tilt and the gravity-model errors on the inertial-system error growth can be estimated by looking for the related growth patterns in the inertially indicated position errors measured during selected time intervals when the aircraft is within a zone of retroreflector coverage.

For the bias type of errors in the position measurements, a process of differencing rather than averaging is needed, since it is not possible to remove the effect of an error whose magnitude changes very slowly with time by the action of averaging.

For errors with a wide frequency spectrum containing bias as well as high-frequency noise, a scheme which reflects both averaging and differencing needs to be adopted, keeping in mind that the process of averaging or integrating a measurement does not aggravate the effect of the bias component, whereas the process of differencing a measurement does aggravate the effect of the high-frequency noise component.

Thus, a judicious balance of averaging and differencing needs to be reflected in an estimation scheme for measurements containing both the bias as well as high-frequency noise components, For such errors, depending upon the complexity of the estimation problem, simpler schemes may be adopted or a more sophisticated approach employing Kalman filtering and smoothing schemes may be needed. The Kalman filtering and smoothing schemes are very powerful techniques using a time-domain approach which readily yields appropriate 
measurement processing algorithms for a given measurement and system model. Thus, much thought and care are needed in modeling the system and measurement schemes during the system-design phase.

(b) Optimal Mixing of Data from The Inertial System and Tracker

We shall briefly illustrate the concepts of filtering and smoothing by considering the estimation of aircraft position using inertial-system data and available tracker data.

The position information from the two sources is complementary in nature with the tracker data occasionally supplying the needed low-frequency information to correct for the low-frequency inertial-system error. The inertial data, on the other hand, have very good high-frequency information which is primarily lacking in the tracker data. The complementary nature of the two types of position information arises due to the different approaches employed in deriving that information. The inertial system derives position information from acceleration measurements, whereas the tracker derives it from range and angle measurements. The need to track the retroreflector while the aircraft is in motion necessarily introduces some lag in the tracking performance. Keeping the platform stable, on which the tracker is mounted, insulates it against aircraft attitude variations during flight, but not against aircraft flight path excursions primarily in the lateral and vertical directions, especially in the presence of atmospheric turbulence. Thus, the tracker-derived aircraft-position information gives only poor definition to high-frequency motion. For the IMU, the instrument errors are primarily of the bias type and the gravity-model errors are mostly unimodal or 
bimodal in nature involving one or two changes in the curvature sign over a typical size 2- by 20-mile survey area, Thus, the inertial system requires at least an occasional position fix and, as the need arises, a fix for the velocity and the IMU coordinate orientation; that need is dictated by the elapsed flight time since last ground-truth fix and the expected magnitude of the instrument biases as well as the curvature of the gravity-model errors over the given flight path.

Figure 4.1-7 illustrates the concepts of filtering and smoothing position measurements derived from the inertial system and tracker. The figure shows the typical growth patterns of the position error with and without estimation for a flight-path segment involving passage through two zones of retroreflector coverage. Curve ABCD shows the inertially indicated position-error growth after an initial fix for position and possibly other biases.

The inertial system error is measured only during aircraft passage through the zones covered by retroreflectors $A$ and $B$; segments $A B$ and $C D$ are relevant thereto. The patterns in the inertial system error growth due to the instrument biases and the average gradient of gravity-model errors are estimated from the a priori estimates of the biases, including the gravity-model error gradient and the position-error measurements. Curve AEFGH represents the realtime estimation-position error that would obtain due to errors in the estimate of the biases. The position errors could be further reduced by smoothing which reflects the better position information available in the coverage zone of the next retroreflector at $B$.

Thus, it may be seen that typically maximum position errors would obtain on the section of the flight somewhere about halfway between two retroreflectors. This observation 


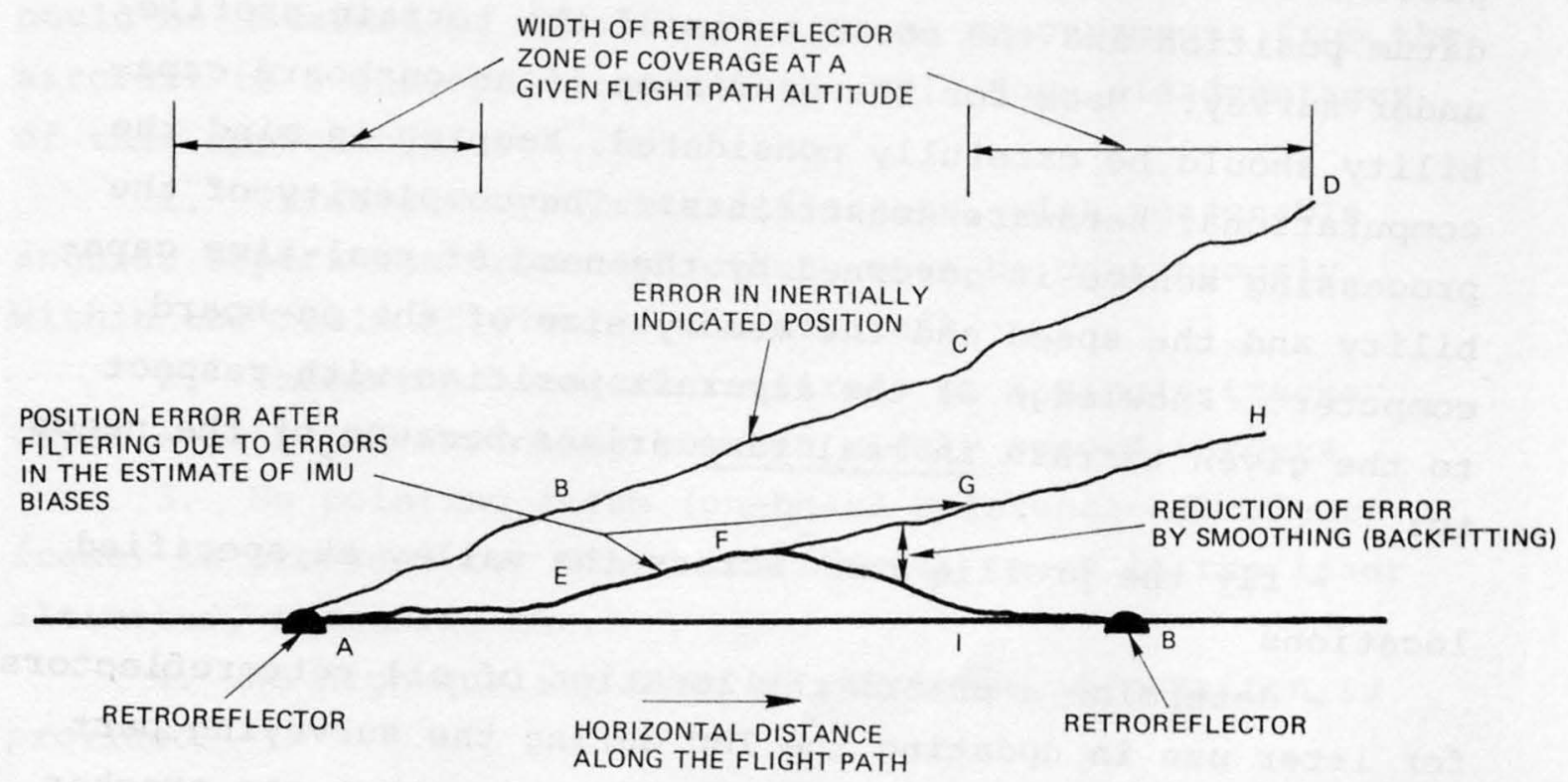

Fig. 4.1-7. Filtering and smoothing of position measurements 
is, of course, valid for a straight flight path between two retroreflectors.

\subsubsection{In-Flight (Real-Time) and Post-Flight Processing}

The processing of measurements could be done as each measurement or batch of measurements becomes available to provide essentially a real-time estimate of the airborne datum position and the coordinates of the terrain profile under survey. Need for the provision of an on-board capability should be carefully considered, keeping in mind the computational hardware constraints. The complexity of the processing scheme is governed by the need of real-time capability and the speed and the memory size of the on-board computer. Knowledge of the aircraft position with respect to the given terrain in real time arises because of the needs to:

- fly the profile runs across the valley at specified locations

- determine a priori the location of all retroreflectors for later use in updating the IMU during the surveying part of the flight; also to determine and ensure that the tracker is locked onto a retroreflector and not an adjoining part of the terrain with good reflectivity

- ascertain that the system is performing satisfactorily

One of the constraints of real-time processing of the measurements is that the position estimates of the airborne datum are based on the present and past measurements only. The position estimates of the airborne datum at a given time, however, could be further refined by smoothing techniques which take into account the future as well as the past measurements in a given survey.

Smoothing thus involves the processing of position measurements - from the tracker and the inertial system - 
over the entire terrain, and thus lends itself to postmeasurement processing which may be best done on the ground, where the processing techniques and their complexity are not limited by computational capability restrictions.

\subsubsection{Aircraft-Position Updating Using The Laser Tracker}

A continuous unambiguous 3-coordinate position fix could be obtained by simultaneous range measurements from the aircraft to 3 surveyed retroreflectors, Some disadvantages of this approach are:

1. Three surveyed retroreflectors, with reasonable angular separation from each other, must be continuously within the field of view of the aircraft

2. Three trackers are required, or a single tracker capable of rapid switching between widely spaced targets

3. No pointing datum (on-board reference-coordinate frame) is provided for other instruments (such as the laser altimeter, a camera, etc.)

4. No high-quality aircraft-attitude information is provided

5. The derived position data are subject to highfrequency instrument noise and signal dropouts

6. The tracking servos must eliminate aircraft motion in the servo loop, since no base-motion isolation is available

7. Velocity information, which may be required to aid the tracking servos, must be derived by the differentiation of position data. Differentiation is a noise-amplifying process.

All of these disadvantages can be overcome, if the laser tracker is used to update and correct an on-board inertial navigator. A single laser tracking a single target at a time, with gaps of no tracking, will be sufficient. It 
is shown in a separate section of this report that the allowed length of the gaps depends principally on the quality of the inertial sensors and the anomalies in the gravity field. In this section the way in which the laser tracker data can be used to accomplish update and correction, when a retroreflector target is in the field of view, is addressed.

The concept of position fixing of a moving vehicle by multiple range measurements to known reference points is a relatively simple one. Radar fixes and DME fixes use this technique, and the geometrical aspects are readily visualized. This is because range measurements are scalar measurements, and the concepts of reference coordinate frames and coordinate transformations do not necessarily enter the problem.

However, the technique to be described here involves vector measurements. This is a fundamental difference, made possible by the presence of an on-board geometric coordinate frame, fixed to the inner gimbal of the IMU, against which the pointing angles of the laser tracker can be referenced. These angles, when combined with the laser range measurement, provide a complete vector measurement, that is, the measurement of the three components of range in a specified coordinate frame, which can be related to a known coordinate frame. The geometric aspects of the problem are not as readily visualized, and resort must be made to analysis.

In Appendix D it is shown that a minimum of three surveyed retroreflectors (not in a line) is required to separately determine aircraft-position errors and platformmisalignment angle errors, and that these targets can be tracked sequentially in the IMU updating process. (It is probably not really necessary to separate horizontal misalignment errors from accelerometer-bias errors.) Because the aircraft is in line of sight of a retroreflector for 
about 50 seconds and each range measurement requires less than about $100 \mathrm{~ms}$, there are at least 500 range measurements per retroreflector in the longitudinal flight. This redundant information can then be averaged to determine the error values of the navigation system and update aircraft position to meet field-survey precision requirements,

\subsubsection{Design of Aerial-Survey Paths}

The flight path of the aircraft making the aerial survey must be designed to minimize the time between retroreflectors and the associated inertial-system updates. By covering the entire survey area with retroreflectors (if a retroreflector were always in line of sight), it would be possible to remove completely the long-term inertial errors and reduce the use of the inertial system to that of removing aircraft "bounces" due to turbulence. However, this is neither desirable nor necessary. We wish to minimize the number of retroreflectors to simplify the conventional surveying work or advance preparations done on the ground. This is particularly true in areas where accessibility may be limited.

The maximum flight altitude for the aircraft is chosen primarily by considering the altitude of the lowest clouds on a cloudy day. Commonly $4000 \mathrm{ft}$. is the lowest altitude. In Sect. 4,1,2,3(b), where the tracker-error sources are examined, the maximum angle between the vertical and the line of sight from the tracker to a retroreflector was shown to be $60^{\circ}$, to stay within the accuracy bounds of $\pm 0.5 \mathrm{ft}$. in the vertical-coordinate direction. This means that the tracker can capture and lock onto a retroreflector when the aircraft is anywhere within a hypothetical inverted cone whose apex is at the retroreflector and whose central angle is $120^{\circ}$. If the aircraft were flown at an altitude of $3000 \mathrm{ft}$. (appropriately below the cloud base), it could 
remain in line-of-sight contact anywhere within a two-mile diameter circle centered on the retroreflector. Increasing the flight altitude slightly would not significantly increase the terrain area for proper line-of-sight contact. Furthermore, the signal-to-noise ratio in both the laser altitude and laser tracker improves in the direction of lowering altitude. Thus, for purposes of simulating a flight mission by computer analysis, the choice of a 3000-ft. maximum flight altitude appears a reasonable compromise.

For a straight two-mile by twenty-mile rectangular survey area with severe gravity anomalies (see section 4.1.6), nine retroreflectors are required to stay within the stipulated error bounds. It is necessary, however, to have only three retroreflectors, not in a straight line (see Appendix D), surveyed on the ground because the remaining six can be "walked-in" and surveyed from the air. This concept minimizes ground-support work yet allows the valley profiles to be flown within the stipulated error bounds. Thus, the requirements of the terrain-profiling system are to measure valley cross sections or profiles at a generalized spacing of $\frac{1}{4}$ mile and to precisely tie in or locate the walked-in retroreflectors.

Two types of flight paths will serve to accomplish these goals, The first type is a single longitudinal pass down the length of the valley to survey the walked-in retroreflectors. The straight flight is accomplished in minimum time and, hence, minimizes the development of any IMU position error between the ground-surveyed retroreflectors. The positions of the unsurveyed retroreflectors can therefore be determined with minimum error. In addition, during the straight-line flight good estimates of IMU drift parameters (gyro drift, misalignment angles, accelerometer bias, etc.) can be made over the time intervals between surveyed 
retroreflectors, In severe gravity-anomaly regions multiple lengthwise passes can be made to measure the anomaly and gravimetric deflection, thereby providing data that can be used to minimize the effects of these error sources.

For short-time intervals the inertial system error growth as a function of time can be calculated using the linear plus quadratic plus cubic time coefficients described in Sections $4,1,2$ and $4,1,3$. Tables $4,1-2$ and $4,1-3$ present the allowable amounts for the various error sources as a function of the number of surveyed retroreflectors, in order to stay within the stipulated survey-accuracy requirements.

In the vertical channel (Table 4.1-2) the linear error source is the $y$ (or $x$ ) tilt-angle error, $\psi_{y}$, multiplied by the velocity in the $x$ direction. The quadratic term is one-half the accelerometer bias, $B_{z}$, and one-half the gravity anomaly, $\Delta G_{Z}$. This assumes a constant gravity anomaly, whereas a real anomaly would have a more convex or ramp-like characteristic (see section 4.1.6). The cubic error term is $\mathrm{g} / 6$ times the change in the anomaly per unit distance. Typical values for normal (Denver) and severe (Des Moines) anomalies are shown.

In the horizontal channels (Table 4.1-3) the linear error term is the azimuth error, $\psi_{z}$, times the aircraft velocity. The quadratic terms are one-half the $x$ and $y$ tilt angles $\left(\psi_{x}, \psi_{y}\right)$ multiplied by $g$, the gravimetric deflection times $\mathrm{g}$, and the accelerometer bias, $\mathrm{B}_{\mathrm{x}}$ or $\mathrm{B}_{\mathrm{y}}$. The cubic terms are g/6 multiplying the gyro drift rate, $\mathrm{R}_{\mathrm{x}}$ or $\mathrm{R}_{\mathrm{y}}$, and the change in gravimetric deflection per unit distance.

The second type flight path of the aircraft is for measurement of the transverse valley profiles and is determined primarily by the particular profiles desired for a given river valley. For the geometry under consideration 
Longitudinal Pass Errors - Vertical Channel

\begin{tabular}{|c|c|c|c|c|}
\hline \multirow[b]{2}{*}{$\begin{array}{l}\text { Number of Surveyed } \\
\text { Retroreflectors }\end{array}$} & \multirow{2}{*}{$\begin{array}{l}\text { Time } \\
\text { Between RR } \\
\text { (Sec) }\end{array}$} & \multicolumn{3}{|c|}{ Allowable Unestimated Bias for $0 . \overline{5} \mathrm{Ft}$ Error } \\
\hline & & $\Psi y \underline{(r a d)}$ & $\mathrm{B}_{\mathrm{Z}}$ and $\Delta \mathrm{G}(\mu \mathrm{g})$ & $\frac{\partial \Delta G}{\partial X}(\mu g / m i l e)$ \\
\hline 1 & 250 & $1.1 \times 10^{-5} *$ & 0.50 & 0.18 \\
\hline 2 & 250 & $1.1 \times 10^{-5} *$ & 0.50 & 0.18 \\
\hline 3 & 200 & $1.9 \times 10^{-5}$ & 1. $38^{*}($ Denver $)$ & 0.83 (Denver) \\
\hline 4 & 150 & $2.9 \times 10^{-5}$ & 3.10 & 2.81 \\
\hline 5 & 71 & $4.0 \times 10^{-5}$ & 6.16 (Des & 7.85 (Des \\
\hline 6 & 51 & $5.6 \times 10^{-5}$ & 11.94 & 21.2 \\
\hline 7 & 36 & $7.9 \times 10^{-5}$ & 23.96 & 60.2 \\
\hline 8 & 25 & $1.1 \times 10^{-4}$ & 49.69 & 179 \\
\hline 9 & 17 & $1.7 \times 10^{-4}$ & 107.46 & 572 \\
\hline 10 & 10 & $2.9 \times 10^{-4}$ & 310.56 & 2811 \\
\hline
\end{tabular}

* Component errors are less than these values 
TABLE $4 \cdot 1-3$

Longitudinal Pass Errors - Horizontal Channels

\begin{tabular}{|c|c|c|c|c|c|}
\hline \multirow{2}{*}{$\begin{array}{l}\text { Number of } \\
\text { Surveyed } \\
\text { Retroreflectors }\end{array}$} & \multicolumn{5}{|c|}{ Allowable Unestimated Bias for $10.0 \mathrm{Ft}$. Error } \\
\hline & $\psi_{\mathrm{z}}(\mathrm{rad})$ & $\psi_{\mathrm{x}, \mathrm{y}}$ or $\phi_{\mathrm{g}}(\mathrm{rad})$ & $\mathrm{B}_{\mathrm{x}}$ and $\mathrm{B}_{\mathrm{y}}(\mu \mathrm{g})$ & $\mathrm{R}_{\mathrm{x}}$ (meru) & $\frac{\Delta \phi}{\Delta x}(\mathrm{sec}) / \mathrm{Mile}$ \\
\hline 1 & $2.1 \times 10^{-4} *$ & $1.0 \times 10^{-6}$ (Denver & 1.0 & .07 & .035 (Denver) \\
\hline 2 & $2.3 \times 10^{-4} *$ & $5.0 \times 10^{-6}$ & 5.0 & .82 & .374 (Des \\
\hline 3 & $3.8 \times 10^{-4}$ & $1.4 \times 10^{-5}$ * & 13.8 * & $3.78 \star$ & 1.74 \\
\hline 4 & $5.8 \times 10^{-4}$ & $3.1 \times 10^{-5}$ & 31.1 & 12.76 & 5.86 \\
\hline 5 & $8.4 \times 10^{-4}$ & $6.2 \times 10^{-5}$ & 61.6 & 35.66 & 16.63 \\
\hline 6 & $1.2 \times 10^{-3}$ & $1.2 \times 10^{-4}$ & 119.4 & 96.21 & 44.15 \\
\hline 7 & $1.6 \times 10^{-3}$ & $2.4 \times 10^{-4}$ & 239.6 & 273.55 & 125.52 \\
\hline 8 & $2.5 \times 10^{-3}$ & $5.0 \times 10^{-4}$ & 496.9 & 816.81 & 374.80 \\
\hline 9 & $4.2 \times 10^{-3}$ & $1.1 \times 10^{-3}$ & 1074.6 & 2597.74 & 1191.99 \\
\hline 10 & $7.5 \times 10^{-3}$ & $3.1 \times 10^{-3}$ & 3105.6 & 12762.70 & 5856.26 \\
\hline
\end{tabular}

* Component errors are less than these values 
a two-mile by twenty-mile valley with transverse profiles one-quarter mile apart is analyzed, Because of the onequarter-mile spacing stipulation, a 2.5 times standard rate turn of the aircraft $(7.5 \mathrm{deg} / \mathrm{sec})$ is employed which results in a turn diameter of one-half mile. The smaller the turn diameter, the less time lost in turning at the edges of the river valley. However, as the turn rate increases, the horizontal accelerations increase and errors in the inertial measurement system grow rapidly. For this reason a 2.5 times standard rate turn is near optimum in working toward the desired one-quarter-mile separation of profiles. Two profiling flights are needed, working from opposite directions and interleaving the first and second sets of transverse profiles to provide the one-quarter-mile separation. Some flight-path details are shown in Figures 4.1-8 and 4.1-9 of Section 4.1 .6 .

The position-error growth of the inertial system during the transverse valley profile measurements can be described by linear, quadratic, and cubic expressions in time for short periods between inertial updates. Although this error growth is the same as for the initial longitudinal flight up or down the valley, the time between retroreflectors (both surveyed and unsurveyed) is greater due to the multiple passes back and forth across the valley. Tables 4.1-4 and 4.1-5 show the allowable bias errors as a function of the total number of retroreflectors. The error sources are identical to those in Tables $4.1-2$ and $4.1-3$, respectively, for linear, quadratic, and cubic time growth.

\subsubsection{Computer Simulation}

Error growth in the IMU was simulated by computer by numerically integrating the differential equations describing the error characteristics. These "error equations" were 
RETROREFLECTOR

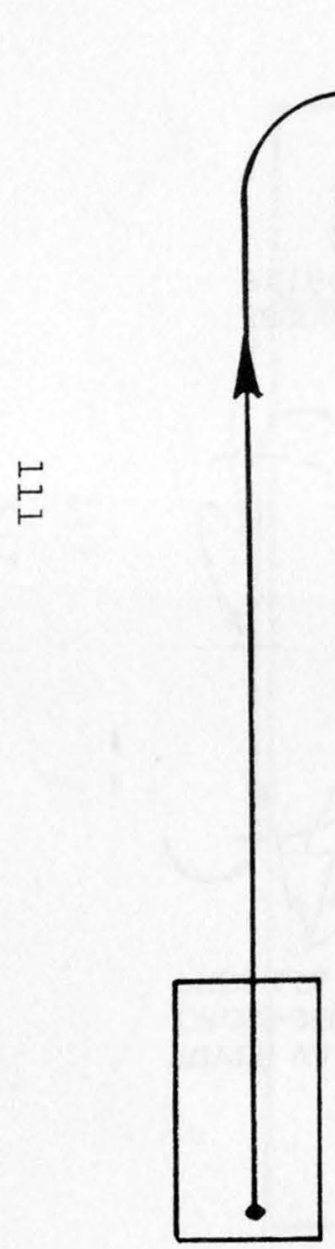

andard

rate turn

$\left(3^{\circ} / \sec \right)$
Taxi and liftoff accelerations

AIRPORT
FIRST

THIRD

RETROREFLECTOR

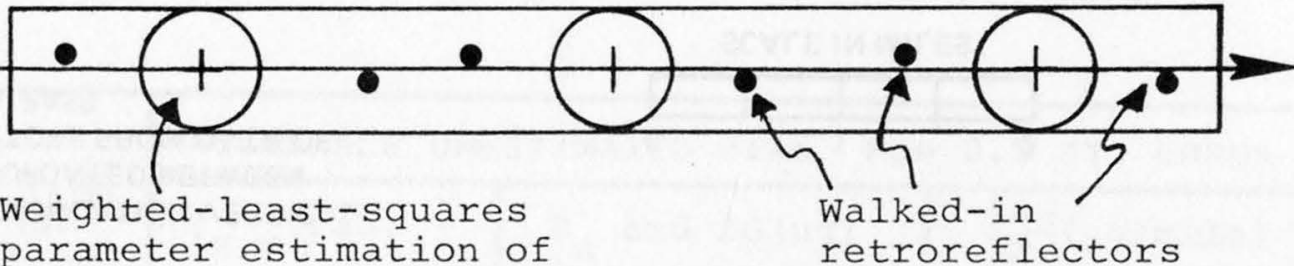

parameter estimation of IMU drift: $\Psi_{\mathrm{x}},{ }_{\mathrm{Y}}, \Psi_{\mathrm{z}}$.

Local level and gyrocompass alignment and IMU calibration

Fig. 4.1-8. Straight flight along river valley 


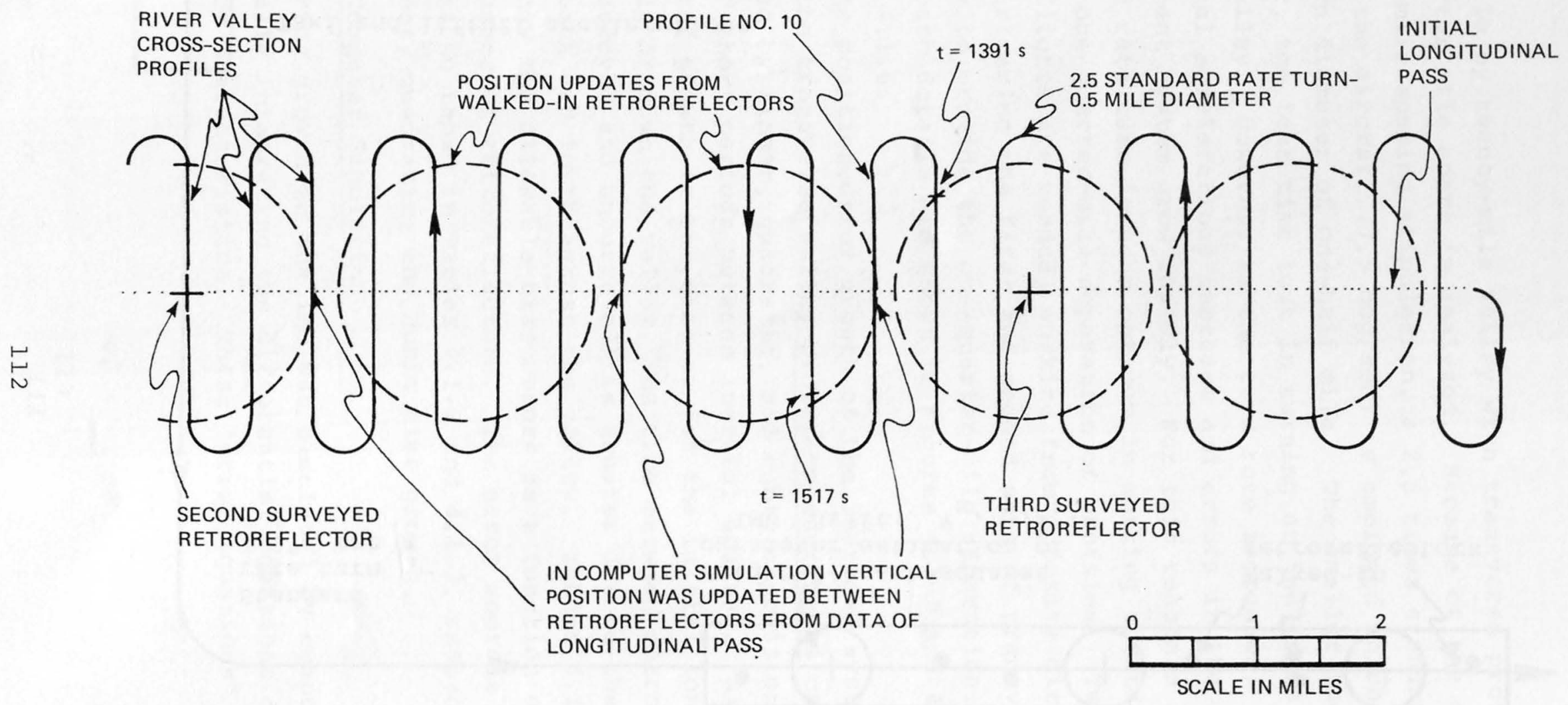

Fig. 4.1-9. Flight to measure transverse cross sections of river valley 
Profile Mode Errors - Vertical Channel

\begin{tabular}{|c|c|c|c|c|c|}
\hline \multirow[b]{2}{*}{$\begin{array}{l}\text { TOTAL NUMBER OF } \\
\text { RETROREFLECTORS }\end{array}$} & \multirow[b]{2}{*}{$\begin{array}{l}\text { MAXIMUM TIME } \\
\text { BETWEEN RR } \\
\text { (SECS) }\end{array}$} & \multicolumn{2}{|c|}{ ALLOWABLE UNESTIMATED BIAS } & \multicolumn{2}{|c|}{ FOR $0.5 \mathrm{FT}$. ERROR } \\
\hline & & $\psi_{x, y}(\operatorname{rad})$ & $\mathrm{B}_{\mathrm{z}}$ and $\Delta \mathrm{G}(\mu$ & & $\frac{\partial \Delta G}{\partial X}(\mu g / m i l e)$ \\
\hline 2 & 1464 & $2.0 \times 10^{-6} *$ & .014 & & $9.0 \times 10^{-4}$ \\
\hline 3 & 881 & $3.2 \times 10^{-6}$ & .040 & & $4.1 \times 10^{-3}$ \\
\hline 4 & 569 & $5.0 \times 10^{-6}$ & .096 & & $1.5 \times 10^{-2}$ \\
\hline 5 & 459 & $6.2 \times 10^{-6}$ & .15 & & $2.9 \times 10^{-2}$ \\
\hline 6 & 370 & $7.7 \times 10^{-6}$ & .23 & & $5.5 \times 10^{-2}$ \\
\hline 7 & 313 & $9.1 \times 10^{-6}$ & .32 & & $9.2 \times 10^{-2}$ \\
\hline 8 & 253 & $1.1 \times 10^{-5}$ & .49 & & $1.7 \times 10^{-1}$ \\
\hline 9 & 227 & $1.3 \times 10^{-5}$ & .60 & & $2.4 \times 10^{-1}$ \\
\hline 10 & 120 & $2.3 \times 10^{-5}$ & $2.15 *(\mathrm{D}$ & & 1.6 (Denver) \\
\hline 11 & 50 & $5.7 \times 10^{-5}$ & 12.4 (Des & & 22 (Des \\
\hline 12 & 42 & $6.8 \times 10^{-5}$ & 17.6 & & \\
\hline
\end{tabular}

* Component errors are less than these values 
TABLE $4.1-5$

Profile Mode Errors - Horizontal Channels

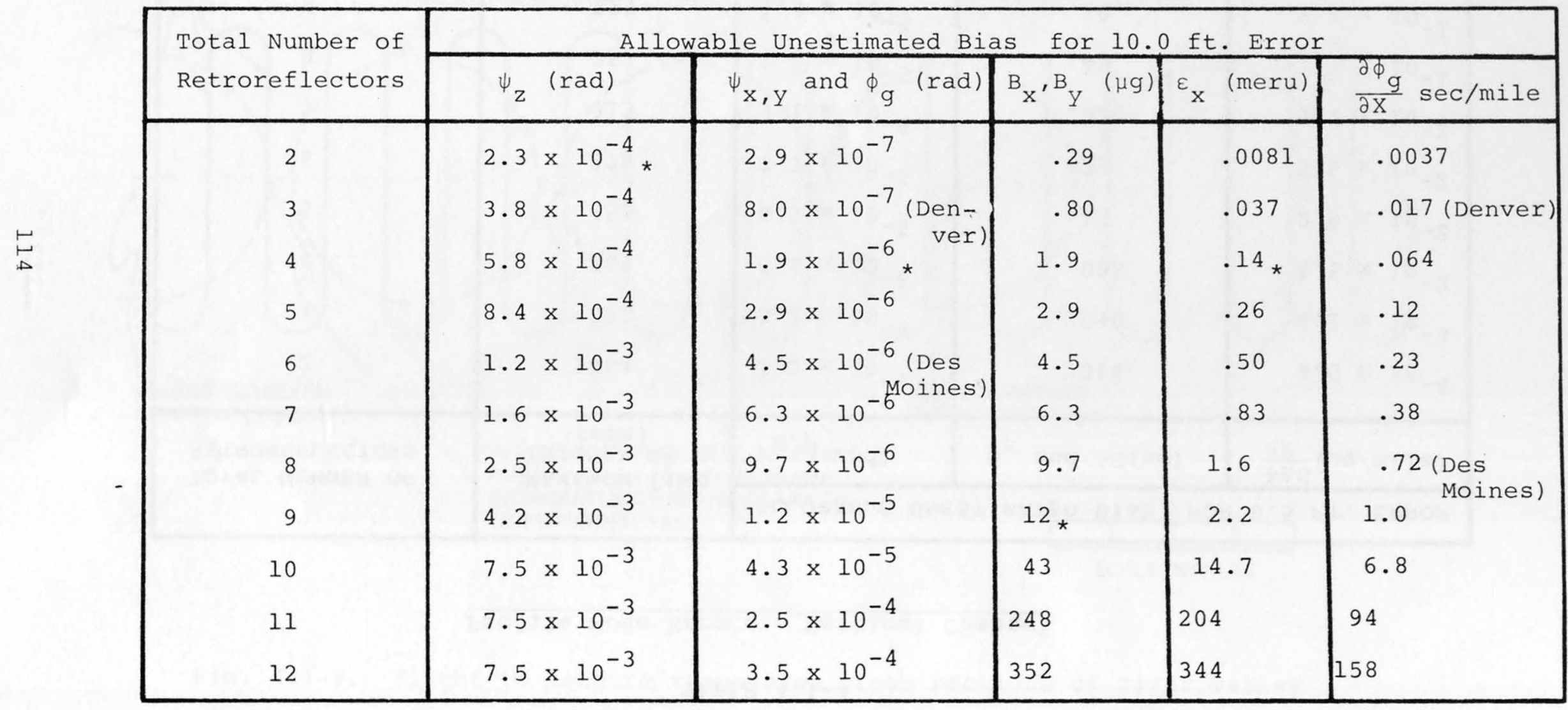

* Component errors are less than these values 
obtained from a first-order perturbation of the equations that determine the true position and true heading of an aircraft. One such equation and a block diagram showing how it is implemented are shown in Appendix E, together with the equations describing error growth.

The results of the numerical integration of the error equations are the position errors in $x$ (North), $y$ (East), and $z$ (down) coordinates. The inputs to the error equations are the takeoff and flight path accelerations; the gyro drift rate, mass unbalance, and torquing errors; the accelerometer bias and scale-factor errors; and the gravity anomalies and vertical deflections within the local area to be surveyed. The computer integrates the differential error equations with a fourth-order Runge-Kutta routine using the input parameters as a function of integration time.

Gravity anomalies were incorporated in the computer program assuming the anomalies and vertical deflections are known at three points in the stream valley, i.e., at the three surveyed retroreflector positions. A straight-line approximation among the surveyed points is then made. For this study the gravity anomaly data for the Des Moines, Iowa, area -- a worst-case situation -- were used. Also included in the simulation were the effects of wind gusts on the inertial-system error.

In the simulation the aircraft is "flown" from an airport 120 miles to the first surveyed retroreflector, where a position correction and a weighted-least-squares estimation of the platform-misalignment angles (angular error) is made. The aircraft then makes a straight 20-mile pass along the length of the river valley surveying-in the "walked-in" retroreflectors and incorporating updates from the second and third surveyed retroreflectors (see Fig. 4.1-8). The aircraft then makes alternating right- and 
left-hand turns to fly the desired transverse cross sections and the laser altimeter collects the profile measurements of the river-valley terrain (see Fig, 4.1-9). Total flight time for such a generalized and idealized mission is two hours and six minutes. A complete description of these procedures and all parameters and coefficients employed in the simulation is given in Appendix E.

A plot of error growth in the IMU is shown in Figure 4.1-10 for the part of the initial 20-mile pass along the length of the river valley between the first and third retroreflectors. (For a detailed explanation of this diagram see Appendix E.5.) This diagram does not include the effects of filtering and smoothing as discussed in Appendices $\mathrm{C}$ and $\mathrm{E}$. Figure 4.1-11 shows the error growth for transverse profile No, 10, between retroreflectors, which results in maximum error buildup. Figure 4.1-11 also does not include the effects of filtering or smoothing.

As can be seen from the error graphs, the errors are close to the allowable margin without filtering or smoothing for this worst case gravity anomaly situation. By including filtering and smoothing it is expected the errors can be reduced to within the error budget as shown in Appendix E.

\subsection{Electroöptical Measurement Systems}

As described in preceding sections of this report, the overall airborne-instrument system will include a subsystem to profile the terrain vertically beneath the aircraft flight path, and a subsystem to track periodically groundcontrol-point targets equipped with retroreflectors. The tracking will be done as needed, when a retroreflector is in the geometrically correct field of view, to update the aircraft position by providing slant range and angle information. Because tracking may occur concurrently with profiling (altitude measuring), two separate instrument subsystems are preferred to accomplish the two distinct types of measurement 


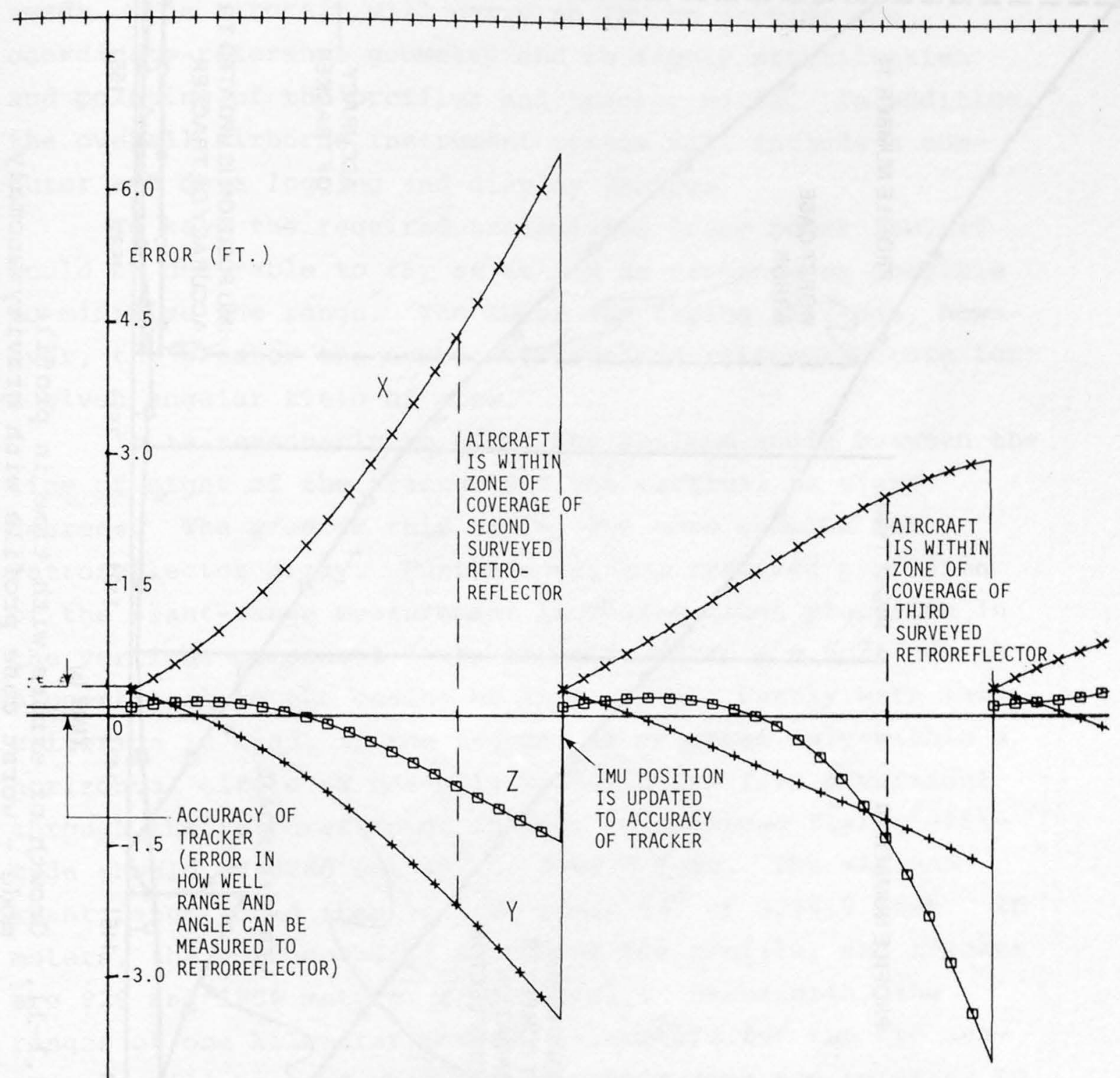

$--4.5$

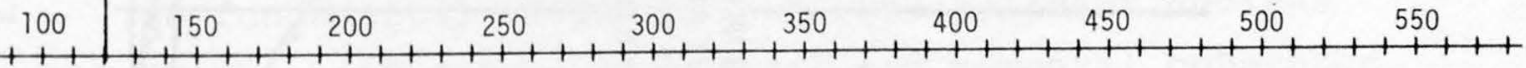

Fig, 4.1-10, Longitudinal pass error growth 


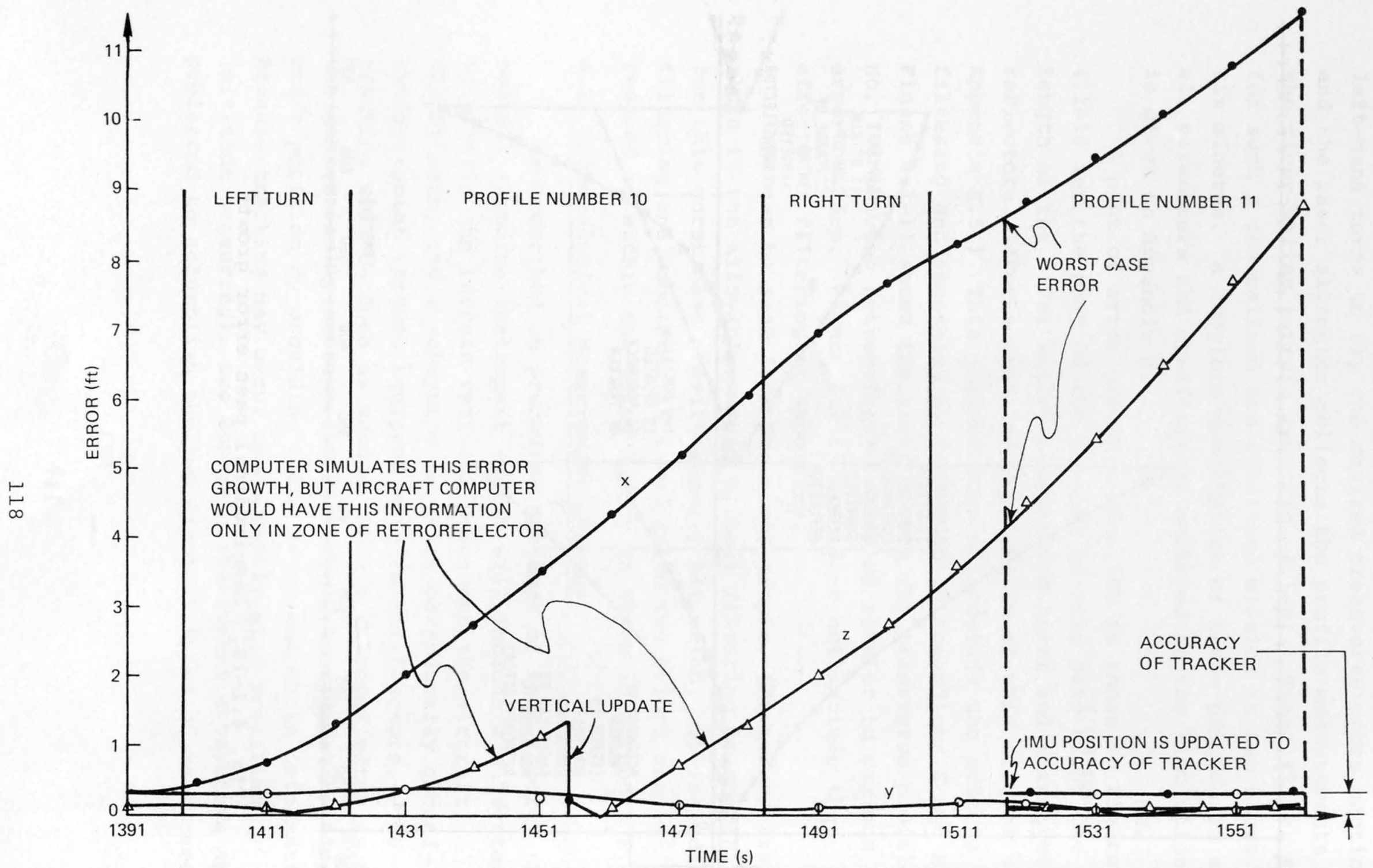

Fig. 4.1-11. Growth of errors with time in profile

mode - worst case profile with gravity anomaly 
needs. The aircraft will carry an IMU to provide the coordinate-reference geometry and to supply stabilization and pointing of the profiler and tracker units. In addition, the overall airborne instrument system will include a computer and data logging and display devices.

To keep the required transmitted laser power low, it would be desirable to fly at as low an altitude as possible to minimize the range. The lower the flying altitude, however, the greater the number of required retroreflectors for a given angular field of view.

It is reasonable to limit the maximum angle between the line of sight of the tracker and the vertical to sixty degrees. The greater this angle, the more complex the retroreflector array. Furthermore, the required precision of the slant-range measurement increases, since precision in the vertical component -- to be held within $\pm 0.5 \mathrm{ft}$. -- is proportional to the cosine of this angle. Partly with this criterion in mind, if the tracker is operated only within a horizontal circle of one-mile radius drawn from a vertical through the retroreflector in use, the maximum flying altitude should be $5280 \cot 60^{\circ}$ or 3048.4 feet. The maximum slant range would then be $5280 \mathrm{cosec} 60^{\circ}$ or 6096.8 feet. In meters, the corresponding ranges of the profiler and tracker are 929 and 1858 meters, respectively. Henceforth, the ranges of one kilometer and two kilometers for the two subsystems will be used when approximate ranges are referred to or used in calculations.

The fundamental advantages offered by laser devices result from a high degree of spatial and temporal coherence. At optical wavelengths, as contrasted with radar wavelengths, the angular resolution can be increased by many orders of magnitude with smaller apertures. The beam divergence can be kept to such low values that it is possible to have the 
target completely intercept the beam. The range equation which normally contains a $\frac{1}{R^{4}}$ dependence, of which $\frac{1}{R^{2}}$ is for the portion of the beam intercepted by the target and $\frac{1}{R^{2}}$ for receiver interception of the return is replaced by $\frac{1}{R^{2}}$ dependence due only to the return.

Since the radiation of a laser beam occurs over a narrow frequency range, it is possible to use narrow band optical filters to discriminate against sunlight "noise".

4.2.1 Choice of Modulation

Before the laser source is selected, the modulation technique must be chosen, since not all lasers may be modulated in any mode desired. Lasers have been used in three modes for the purpose of ranging. The first method is the basic pulse technique in which a narrow pulse (usually less than $50 \mathrm{nsec}$ ) is transmitted and the transit time to the target and return to the receiver is proportional to the range... The second technique utilizes an amplitude modulated continuous wave (CW) laser, The beam is directed at a target and the return signal will have its phase shifted proportional to range... The third technique is the interferometer method, in which the frequency of the $\mathrm{CW}$ laser itself is used. (7) Interferometric techniques can be ruled out for this application since they are generally limited to short distances of up to 50 meters. Both the first and second techniques are possible at the ranges under consideration.

\subsubsection{Pulse Modulation}

Systems using pulse modulation are commonly referred to as optical radars. Range information is obtained by measuring the propagation time of a light pulse from the source to the target and back to the receiver. The accuracy of the measurement depends on the definition in time of the 
transmitted pulse and on the resolving capability of the photodetector and the time-measurement system.

\subsection{Advantages of Pulsed Modulation Systems}

Pulse techniques are attractive because no complex optics or heterodyning are required. The average power of a pulsed system is low since with narrow pulses, the duty cycle is extremely low, Range-gating techniques may be employed, if the approximate distance of the target is known, providing background noise elimination for most of the duration of the time interval between transmitted and received pulses.

If a laser beam hits more than one target, a pulsed system offers some unique advantages. Such a situation may occur when the airplane is flying over tree branches or sparse vegetation and part of the beam hits branches, but part also hits the terrain (see Fig. 4,2-1). A simple technique can detect the last pulse - the one which hits the terrain. Two counters are enabled simultaneously upon the emission of the transmitted laser pulse. They are both set to terminate at a predetermined time, which is the termination of the range gate. The duration of the range gate is controlled by a priori knowledge of approximate altitude. In most cases, where the interfering object is a significant distance from the ground, the range gate will be sufficient to discriminate between unwanted return pulses. In Fig. 4.2-1 it is assumed that more than one return pulse is received during the range-gate interval. Each time a pulse is received, Counter 2 is instantaneously cleared and immediately starts counting again. The count in counter 2, at termination of the range gate, subtracted from the count in Counter 1 , is the range to the terrain.

Since the time measurement is $2 \mathrm{~d} / \mathrm{c}$, where $\mathrm{d}$ is the 


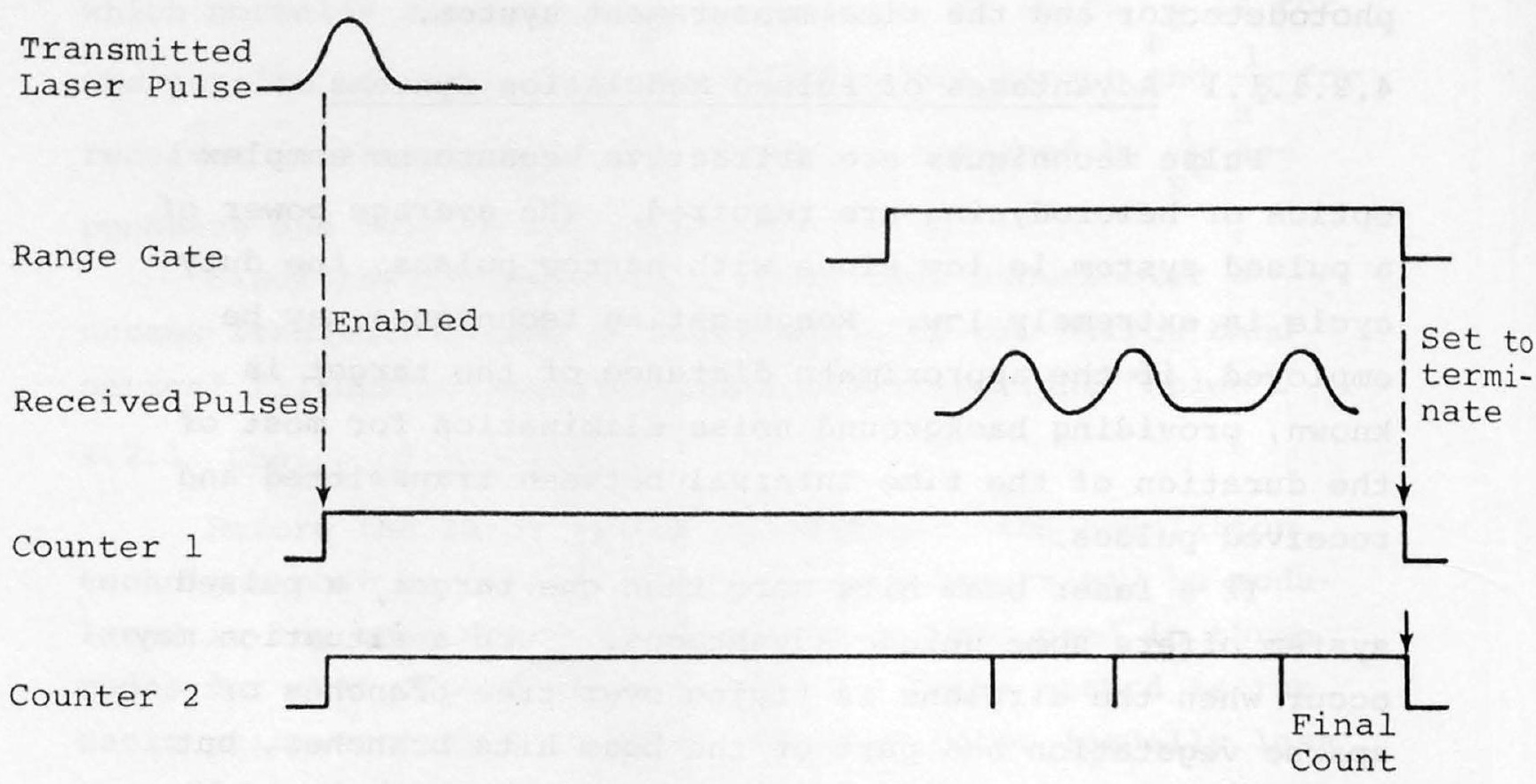

Counter $1-2$

\section{Fig. 4.2-1. Last pulse selection}

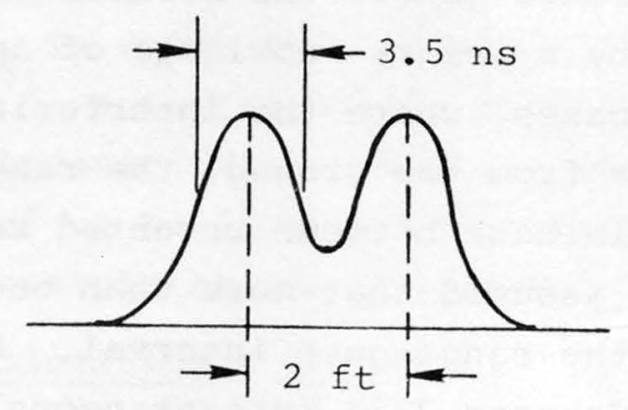

Fig. 4.2-2. Resolution of pulsed lasers 
range and $\mathrm{c}$ is the speed of light, the vertical accuracy stipulation of $0,5 \mathrm{ft}$. corresponds to one nanosecond (ns). This puts stringent requirements on the switching times of enabling and clearing the counter, if accuracies on the order of 0.5 foot or better are required,

The width of the laser pulse basically determines the resolution of the targets. In Fig. 4.2-2, the return pulses from two targets two feet apart are shown. The transmitted pulse is $3.5 \mathrm{~ns}$ wide, has a 1 ns rise time, and is emitted from a single gallium-arsenide (GaAs) injection laser. The targets in this case are retroreflectors, The return from non-cooperative targets would not be as ideal as shown in Fig. 4.2-2. The pulses would probably be widened at the trailing edge and would be distorted.

Signal-to-noise ratios as a function of pulse rise time and pulse width for a desired time delay error are given in Appendix F,

\subsection{Disadvantages of Pulsed Systems}

If a brute force time interval measurement were to be made, the rise times of the transmitted and received pulses would have to be maintained to a one nanosecond order of magnitude. Time-interval measurements would have to employ analog interpolation techniques, since clocks in excess of 100 to $200 \mathrm{MHz}$ would be difficult to obtain with available integrated circuits, Extreme care in design and packaging would be needed to minimize capacitance loading of the pulses. Although lasers with picosecond-duration pulses are available, they are usually large and bulky and require cooling in order to obtain high pulse-repetition rates required for high data-rate systems. For the envisioned profiler, the shape of the received pulse would be significantly distorted due to atmospheric and target scintillations, 
so that all received energy would have to fall in a time interval whose duration is less than the minimum time delay error. For the envisioned tracker a much stronger return would be obtained from the retroreflector. Although automatic half-maximum detection (detecting the point at which the received pulse rise time reaches half of its maximum amplitude independent of variations in amplitude or pulse width stretching) might be expected to be more successful in this application, variations due to the angle of incidence of the beam with respect to the retroreflector assembly and atmospheric conditions may still cause significant distortions in the shape of the rise time of the reflected pulse. When measuring time durations in the subnanosecond region, the stability of the propagation delays in the electronic measuring circuits as a function of temperature, humidity and time becomes significant so that repeated calibrations of the electronics might be necessary to minimize this error. For a compact pulsed laser system, gallium arsenide injection lasers are the most attractive. They are small in size and are easily pulse modulated, An array of diodes is necessary, however, for powers above a few watts. Since it is difficult to obtain simultaneous lasing from the individual diodes in an array, typical pulse widths of commercially available arrays are limited to about 100 to 200 nanoseconds at high peak powers and the pulse repetition rate has to be kept moderately low to avoid high-average power. In a moving airplane, therefore, integration over a number of pulses to improve signal-to-noise ratio would not be very effective.

Since a ranging precision of $\pm 0.5 \mathrm{ft}$. or better requires pulse measurements in the subnanosecond area, it can be concluded that a pulse-type laser would require exotic measurement techniques which stretch the state of the art. 
Circuit techniques which define the half-max point on the rise time of the pulse or determine precisely the centroid of the pulse have been used but are extremely difficult. Recently, a technique ${ }^{5}$ involving an image converter tube equipped with deflection plates was used to make a precise measurement of the fractional amount by which the time of flight of the ranging pulse exceeds an integral multiple of the period of a stable oscillator. The method involves the measurement of the arrival of four pulses of light, two of which are optically delayed. A precise determination of the magnitude of a fraction of a period is made from the relative positions of the known segments on the output phosphor, produced by the transmitted pulse, and those produced by the reflected pulse.

In summary, it is apparent that pulse techniques for the accuracy required are extremely difficult. If subnanosecond pulses were available from gallium arsenide lasers, perhaps more serious consideration would be given to pulse methods.

\section{2,1.2 Sinusoidally Modulated Beam Techniques}

When the beam of a continuous wave (CW) laser is modulated sinusoidally and directed at a target, the return beam will have its phase shifted proportional to the range. Such modulation can be described as either "intensity", referring to the light, or "amplitude", referring to the power.

The power transmitted in such a beam, at a frequency $f$, is $P_{t}=P_{0}(1+m \cos 2 \pi f t)$, where $P_{0}$ is the average value of power and $m$ is the modulation index. The power in the reflected beam is

$$
P_{r}=\alpha P_{0}\left[\left(1+m \cos 2 \pi f\left(t-\frac{2 d n g}{c}\right)\right)\right]
$$


where $\alpha$ is a constant attenuation coefficient due to propagation effects of optical lasers, $d$ is the one way distance (range) the beam travels, $c$ is the velocity of light, and $n_{g}$ is the average value of the group refractive index along the optical path for the wavelength used. Range information is obtained by electronically determining the phase difference between transmitted and received beams.

$$
\theta=\frac{4 \pi f d n_{g}}{c}
$$

Phase-difference measurements can be made that are accurate to at least one part in 1000 by a method of digitization to be explained below. To determine the frequency of the $\mathrm{CW}$ to be employed in this measurement, rearrange Eq. $(4 \cdot 2-2)$.

$$
f=\frac{c \theta}{4 \pi d n_{g}}
$$

Assuming a resolution of $0.1 \mathrm{ft}$. is desired (to insure overall accuracy of $0.5 \mathrm{ft.}$ ), a modulation frequency corresponding to $1000 \times 0.1=100$ feet can be computed. Phase measurement is unambiguous only up to a phase difference of $2 \pi$ radians, which here corresponds to $100 \mathrm{ft}$. Therefore, $\theta=2 \pi$. Let $d=100 \mathrm{ft}$.

$$
\begin{aligned}
f & =\frac{299,792,500 \frac{\mathrm{m}}{\mathrm{sec}}}{2(100)} \times 3.280829895 \mathrm{ft} / \mathrm{m} \\
& =4.917856 \mathrm{MHz}
\end{aligned}
$$

This result is shown in the second line of Appendix $K$. The effect of the refraction index of air is neglected. 
To resolve the ambiguity of either $100 \mathrm{ft}$, or a greater range, one or more lower frequencies can be used. By dividing the modulation frequency by 10 , an unambiguous reading will be obtained every 1000 feet (see Appendix $K$ ). Dividing by 100 permits an unambiguous reading every 10,000 feet. Since quantizing a range of 10,000 feet to one part in 1000 will provide a resolution of $10 \mathrm{feet}$, the use of two modulation frequencies, sequentially, corresponding to 100 feet and 10,000 feet should suffice for both the profiler and tracker applications, A higher frequency of $50 \mathrm{MHz}$, as shown in the first line of Appendix $K$, makes possible resolution of $0.01 \mathrm{ft}$. At this frequency, the signal-to-noise ratio for a measurement of \pm 0.1 foot is ten times greater than at $5 \mathrm{MHz}$.

With a 5 or $50 \mathrm{MHz}$ modulation frequency, a phase measurement to one part in 1,000 requires that the frequency of the clock for range digitization be in the thousands of $\mathrm{MHz}$ (see Fig. 4.2-6). A convenient method to avoid this is to frequency down-convert both the transmitted and received signal. A local oscillator at a frequency, $f_{1}$, feeds two mixers. The other inputs to the mixers are a portion of the transmitted signal and the received signal, respectively. The outputs of the mixers are now at a frequency $f-f_{I^{\prime}}$ called the intermediate frequency (IF). The phase difference between the two IF signals at the outputs of the mixers is the same as that of the signals at frequency $f$. Frequency down-conversion to the $\mathrm{KHz}$ range allows for convenient phase measurements using a realizable clock in the $\mathrm{MHz}$ range. This process of frequency conversion is also called mixing, heterodyne action and beating.

With an aircraft traveling about $175 \mathrm{ft} / \mathrm{sec}$ (120 mph), the time it would take to fly one foot would be about $5.7 \mathrm{~ms}$. A smoothing bandwidth of $175 \mathrm{~Hz}$ would be adequate for this 


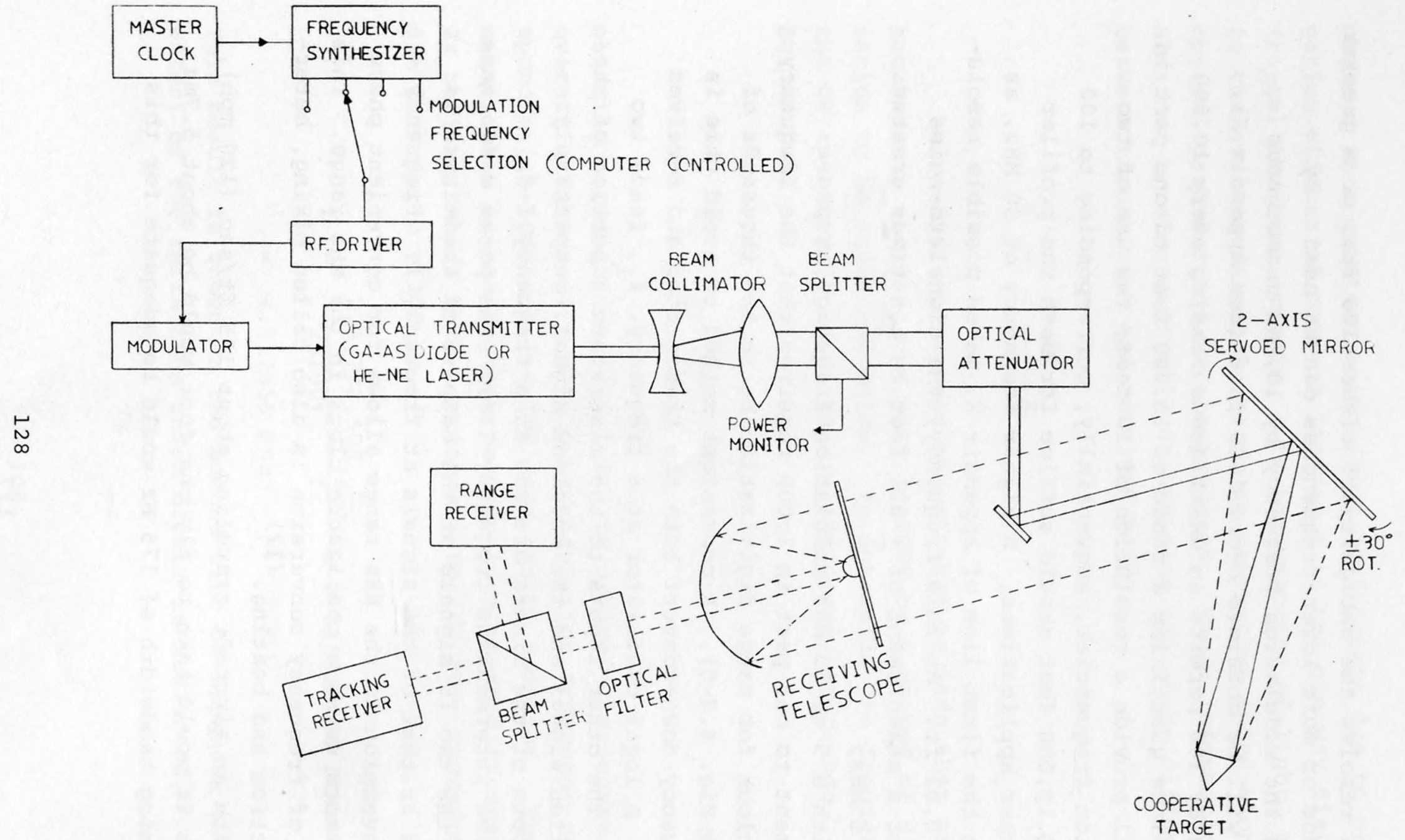

Fig. 4.2-3. Optical tracker 
type of measurement. If the IF frequency is selected to be about a $\mathrm{kHz}$, the phase measurement is sampled at the IF frequency or an integral submultiple of it. A $500 \mathrm{~Hz}$ sampling rate would be adequate for a bandwidth of $175 \mathrm{~Hz}$. Narrow bandwidths would give improved filtering characteristics and a better signal-to-noise ratio but a poorer horizontal resolution. For example, a $50 \mathrm{~Hz}$ bandwidth corresponding to a 20 millisecond time constant would provide only about $3.5 \mathrm{ft}$. resolution at a speed of $175 \mathrm{ft} / \mathrm{sec}$.

The local oscillator frequency is selected so that for each modulation frequency, the IF frequency is an integral submultiple of the modulation frequency. This is not actually $5 \mathrm{KHz}$ but is the subharmonic, $4.916 \mathrm{KHz}$,

A clock for range digitization of $5 \mathrm{MHz}$ would provide a resolution of one part in 1000 and $50 \mathrm{MHz}$ would provide one part in 10,000. Because the continuous-wave (CW) system has a narrow bandwidth, however, in the case where the same beam hits more than one target, the resolution is not as good as it might be with a pulsed system, A narrow bandwidth system acts like a low-pass filter, and cannot follow sudden changes in phase which would occur.

Intensity modulated lasers have been used extensively in surveying, ranging and tracking applications.

\subsection{Effects of Air-Density Variations}

The uncertainty in the average propagation velocity of the radiation resulting from the inhomogeneity of the atmosphere is one of the main limitations to the accuracy of range measurements using intensity modulation. When the ultimate in accuracy is required, the dispersion method is used.

Simultaneous measurements of optical path length at two widely separated wavelengths are used to determine the 
average refractive index over the path and hence the true geometrical distance. Systems using this method have been successfully built.

The value of the group refractive index is given by $\mathrm{n}_{\mathrm{g}}-1=284.56 \times 10^{-6}$ at $6328 \AA$, the wavelength for helium neon lasers, At $9000 \AA$, the wavelength of gallium arsenide lasers, $\mathrm{n}_{\mathrm{g}}-1$ is approximately $274 \times 10^{-6}$. In order to take this into account, the computed modulation frequency is modified by this factor.

$$
f^{\prime}=\frac{4.917856}{1.00028456}=4.91646 \mathrm{MHz}
$$

for the helium neon wavelength.

The refractive index variation with presssure and temperature is given by:

$$
\mathrm{n}_{\mathrm{air}}-1=\left(\mathrm{n}_{\mathrm{g}}-1\right) \frac{288}{760} \frac{\mathrm{P}}{\mathrm{T}+273}
$$

The group refractive index as a function of wavelength is given by Ref. 6 :

$$
\left(h_{g}-1\right) 10^{8}=27260+460.8 / \lambda^{2}+6.60 / \lambda^{4}
$$

where $\lambda$ is in microns.

This relationship is true for the atmosphere at $15^{\circ} \mathrm{C}$, $760 \mathrm{mmHg}$ pressure and containing $0.03 \%$ by volume of carbon dioxide. The effect of water-vapor content is very small, contributing less than half a part in a million to the refractive index. The relative error in determination of index of refraction and distance is, by Ref. 6 , only $\pm 1.0 \times 10^{-6}$ for $\mathrm{a} \pm 1.0^{\circ} \mathrm{C}$. temperature change and $\pm 0.4 \times 10^{-6}$ for $\pm 2.6 \mathrm{mmHg}$ pressure change. Thus, to stay within a 0.1 foot error at 6000-foot range, a temperature change of over $16^{\circ} \mathrm{C}$. 
could be tolerated.

Probably the vertical gradient of the index of refraction $\frac{\mathrm{dn}}{\mathrm{d} z}$ contributes the greatest error. It has a typical value of $-30 \times 10^{-6} / \mathrm{km},[7]$ Although the errors appear to be small, the air pressure and temperature should be measured to correct for the index of refraction. The decrease in the index of refraction of air with increasing altitude results in a curvature of the light rays from and to the laser tracker. This produces a small pointing error in the laser tracker and a corresponding error in the laserprofiler position. The pointing angular error can be directly calculated, and for the worst case of $60^{\circ}$ elevation angle and 6000-ft. slant range, the angular error is $3.2 \mathrm{sec}$. This error can be largely eliminated by calculating and applying a correction in real time during the flight.

If two different wavelengths are used for the tracker and profiler (GaAs - $9040 \AA$ and HeNe - 6328 $)$, the dispersion method may be used to advantage. When the aircraft is flying directly over a retroreflector, the optical paths for the tracker and profiler will be the same. (Indication that the profiler is over the retroreflector can be obtained from the automatic gain control (AGC) loop in the receiver, indicating a strong signal return.)

Since $\left(n_{g}-1\right)$ at a given wavelength is proportional to air density for dry air, the difference in refractive index, and hence, the difference in transit time for the two signals will be proportional to the average air density over the path. A measurement of the difference in transit times can thus be used to give the average density over the path. From this quantity the average refractive index for either wavelength may be calculated, providing the desired correction for the distance measurement. 
The true distance, D, may be computed from

$$
D=D_{1}-K\left(D_{2}-D_{1}\right) \quad[\text { Ref. 6] }
$$

where

$$
\begin{aligned}
& K= \frac{n_{1}-1}{n_{2}-n_{1}} \text { and } n_{1} \text { and } n_{2} \text { are the corre- } \\
& \text { sponding refractive indices, and } D_{1} \text { and } D_{2} \\
& \text { are the measured optical paths for the two } \\
& \text { wavelengths. }
\end{aligned}
$$

\subsubsection{Considerations for Choice of Laser}

Since the $\mathrm{CW}$ laser with intensity modulation appears to provide the simplest method of achieving the range measurement accuracy required, the choice of lasers is limited to those that can be modulated in this manner.

For an aircraft application, size, weight, power and reliability considerations are of prime importance. It is desired to avoid cooling apparatus, gas filling and sophisticated environmental control. Even before performing calculations, the choice can be narrowed down to the most promising possibilities, Helium neon is probably the simplest and most reliable of the gas lasers. Based on its use in other systems, such as the Spectra-Physics Geodolite 3A altimeter, the available power from helium-neon lasers is more than sufficient. These lasers, however, are long and bulky. Ion lasers, such as argon and krypton, have more than adequate power, but require water cooling and do not have the reliability of the helium-neon laser. Many of the commercial range-finding instruments use a gallium arsenide diode. These are low-power devices which are used with a cooperative target. They probably would not suffice for the profiling application where the range is measured to a 
diffuse surface. For the tracker application, however, even though the maximum range to be measured is twice that in the profiler, the strong return from a retroreflector may make the use of such a device possible.

The choice of wavelength is another consideration in selecting lasers, Reflectivity of most surfaces goes up with wavelength, although water-surface reflectivity goes down. The blue-green wavelengths are favorable for measuring the bottom of water bodies since the penetration of water is quite good at these wavelengths. The quantum efficiency of photomultipliers is greater at the visible wavelengths than in the infrared. Atmospheric attenuation due to scattering is also a function of wavelength. In the visual and infrared regions, Mie scattering dominates and Rayleigh scattering can be neglected. The atmospheric attenuation due to scattering increases with wavelength. The wavelength of helium neon, $6328 \AA$, is a fairly favorable wavelength when all factors are taken into account.

\subsubsection{Optical Heterodyning}

Because lasers emit narrow spectral lines, it is possible to obtain mixing action at optical frequencies. optical heterodyning or photomixing of a weak received signal with a strong reference or local oscillator signal serves to eliminate the internal noise of the detector from consideration in the signal-to-noise ratio. Also, since the bandwidths of the IF are much narrower than optical filters, the effects of background noise are drastically reduced.

optical heterodyning, however, requires that the signal and reference beam remain collinear until they enter the detection aperture of the system. Severe limits are put on the field of view of the receiver and the stability of the laser local oscillator. Since the optical design of the 
system is complicated by the use of optical heterodyning, a direct detection system is usually employed for most rangemeasurement systems of the type under consideration and the heterodyning occurs after the signal has been received from the photodetector.

\subsubsection{Range Calculations}

The background noise is given by:

$$
\mathrm{P}_{\mathrm{b}}=\mathrm{H}_{\lambda}{ }^{\mathrm{A}}{ }_{\mathrm{r}} \Omega_{\mathrm{r}} \Delta \lambda \mathrm{T}_{\mathrm{O}} \frac{\mathrm{P}_{\mathrm{b}} \mathrm{T} \mathrm{a}}{\pi}+\frac{1}{4 \pi}
$$

where

$$
\begin{aligned}
& { }_{\lambda}=\text { spectral irradiance in watts } / \mathrm{m}^{2} \AA \\
& \Omega_{r}=\text { receiver field of view in steradians } \\
& { }_{\mathrm{A}}=\text { area of receiver collecting optics } \\
& \Delta \lambda=\text { optical bandwidth in angstroms } \\
& \mathrm{T}_{\mathrm{O}}=\text { transmittance of optics } \\
& \mathrm{T}_{\mathrm{a}}=\text { transmittance of atmosphere } \\
& \mathrm{P}_{\mathrm{b}}=\text { average radiant reflectance from a }
\end{aligned}
$$

The first term of Eq. (4.2-7) describes the contribution of noise power due to reflected background radiation at the target. The target, which is the surface of the terrain, is assumed to be a diffuse reflector and is assumed to fill the beam. The second term gives the amount of background radiation that is scattered in the atmosphere toward the receiver.

It would be desirable to use a CW gallium arsenide laser because of its small size, efficiency and ease of modulation. At present gallium arsenide lasers in the Laboratory are capable of about 5 to $10 \mathrm{mw}$ of power. They are 
expected to be commercially available soon. Calculations are performed at $9000 \AA$ (gallium arsenide) for the tracker and $6328 \AA$ (HeNe) for the profiler to see if it is feasible to obtain the necessary power at a flying altitude of approximately one kilometer for the profiler and at a slant range of up to two kilometers for the tracker. An 8-inch diameter receiving aperture is assumed for the profiler and one-inch for the tracker. A $0.1 \mathrm{mr}$ transmitter beam is assumed for the altimeter (yielding a $0.3-\mathrm{ft}$. spot from $3000 \mathrm{ft.}$ ). The tracker will have a ten-foot spot size.

The calculations for background noise are shown in Appendix G. The background noise for the HeNe profiler is determined to be $3.6 \times 10^{-11}$ watts, and for the GaAs tracker it is $2.34 \times 10^{-9}$ watts. The corresponding noise currents are $7.3 \times 10^{-13}$ amps and $5.10 \times 10^{-12}$ amps, respectively.

In Appendix $H$ the signal-to-noise $\left(\frac{\mathrm{S}}{\mathrm{N}}\right)$ ratios are derived as a function of the uncertainty in range. With a $50 \mathrm{MHz}$ modulation frequency (10 ft. range), the required $\frac{\mathrm{S}}{\mathrm{N}}$ ratio is 11.25. A photomultiplier detector is assumed and an expression for received power as a function of $\frac{\mathrm{S}}{\mathrm{N}}$ ratio is developed.

$$
P_{r}=\frac{h \nu}{n e}\left[2 \frac{S}{N} \text { eB }\left(I_{b}+I_{d}\right)\right]^{\frac{1}{2}}
$$

The bandwidth used is $200 \mathrm{~Hz}$ which should be more than adequate (see Sec. 4.2.1.2). The received powers computed are $1.13 \times 10^{-12}$ watts for the profiler and $3.28 \times 10^{-11}$ watts for the tracker.

Equations for power received by the altimeter and tracker are developed in Appendix I, For the altimeter, it is shown that in the case where the target is equal to or larger than the beamwidth and the receiver field of view is greater than, or equal to, the transmitter beamwidth, the equation for power received is 


$$
P_{r}=\frac{P_{t} \rho T_{r} \varepsilon^{-2 \sigma r_{A}}{ }_{r}}{\pi r^{2}}
$$

For the tracker, when the return beam is larger than the aperture of the receiving telescope,

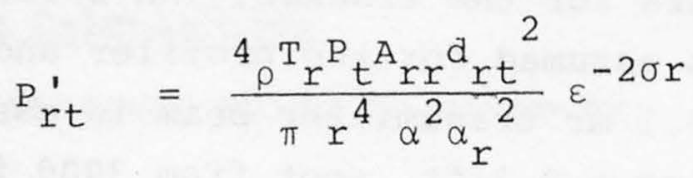

The values of $\sigma$, the atmospheric extinction coefficient, are also developed in Appendix I. In Appendix $J$ the transmitter power is determined using the power equations. For the profiler, using a HeNe laser the required transmitter power is $20.8 \mathrm{milliwatts.} \mathrm{If} \mathrm{calculations} \mathrm{were} \mathrm{performed} \mathrm{at}$ the gallium arsenide wavelength, it would be found that the required power would be an order of magnitude greater due to larger optical bandwidth, reduced quantum efficiency of the photomultiplier and higher dark current. Gallium arsenide CW devices, at present, are limited to approximately 10 milliwatts so that they would not be feasible for the profilter application.

Typical lengths of HeNe lasers are 18 inches for $5 \mathrm{mw}$ power and 32 inches for 15mw. The spectra-Physics Geodolite uses 30 and $40 \mathrm{mw}$ lasers with 5-angstrom optical bandwidths. These instruments can range over 10,000 feet on a clear day. on a clear day only about llmw would be needed at 3000 feet. In addition, effects due to atmospheric turbulence are not accounted for in the range equation. Some of these effects include beam scintillation, image dancing, spatial coherence degradation and beam steering.

For the tracker the required transmitter power is computed to be $2.25 \mathrm{mw}$. It is seen that a gallium arsenide 
diode is adequate for the tracker application. For poorer retroreflectors and/or higher altitudes, a larger aperture receiver is required. The main advantage of gallium arsenide is that it is small and easy to package and modulate. Also, the use of a different wavelength than that used in the profiler may provide some means for atmospheric index of refraction correction. Alternatively, a one-milliwatt (or less) HeNe laser can be used. This would provide for more similarity with the profiler and the design problems could be attacked in parallel.

\subsubsection{Tracking Receiver}

In Fig. 4.2-3, a block diagram of the optical tracker is given. The transmitter portion of the diagram will be very similar for both the tracker and altimeter. In the altimeter there is no necessity for a servoed mirror, since the beam will always be directed vertically downward.

For the tracker, however, a servoed mirror is shown. The mirror will have $\pm 30^{\circ}$ rotation so that the transmitted beam may have $\pm 60^{\circ}$ rotation. This freedom of rotation will be supplied on two axes. The receiver is shown co-axial with the transmitted beam to the servoed mirror. Torque motors will slew the mirror upon computer command in both the search and track mode. In the search mode, a simple back-and-forth scan may suffice in one axis, while the aircraft's forward motion will provide an effective resultant sawtooth pattern to the beam. A 10-foot spot diameter will be used in the search mode. The scan rate must be fast enough so that the target will not be missed while undergoing a search pattern. Once acquisition of the reflector has occurred, the system will revert to a track mode and the spot diameter will be reduced to one foot,

The transmitted beam is shown at approximately $15^{\circ}$ 
below the horizontal in order to minimize the ellipticity of the beam spot as it hits the servoed mirror. The axis of the receiving telescope is shown co-axial with that of the transmitted beam. With use of a servoed mirror, the accuracy of the angular readouts must be about $10 \mathrm{sec}$. to achieve 20 sec, system accuracy, since the mirror only moves through half the angle that the beam moves.

It may be possible to avoid the use of a servoed mirror. If the transmitter is lightweight (which will be the case with a CW GaAs diode), the whole transmitter receiver assembly may be slewed through $\pm 60^{\circ}$, if the receiving optics and mechanical assembly can be made lightweight.

In the tracker, the output of the photodetector feeds a tracking receiver and ranging receiver, The ranging receiver will be similar to the one used in the profiler. Two of the ways in which tracking receivers may be implemented include a four-quadrant detector (Fig, 4.2-4) and an image intensifier tube (Fig. 4.2-5). The image intensifier has a small instantaneous field of view, but requires a continuous electronic scan for centering of the image.

\subsubsection{Range Measurement Using Intensity Modulation}

A block diagram of the ranging receiver is given in Fig. 4.2-6. As described in Sec. 4.2.1.2, a direct detection system is used. A portion of the transmitted signal is sent to a reference mixer, while the received light after photodetection is sent to the signal mixer. The local oscillator supplies the reference signal to both mixers. The local oscillator frequency is selected in tandem with the modulation frequency so that a constant IF frequency is always obtained. The signal IF is fed to a phase-locked loop where it is further filtered. The phase of the output of the voltage- 


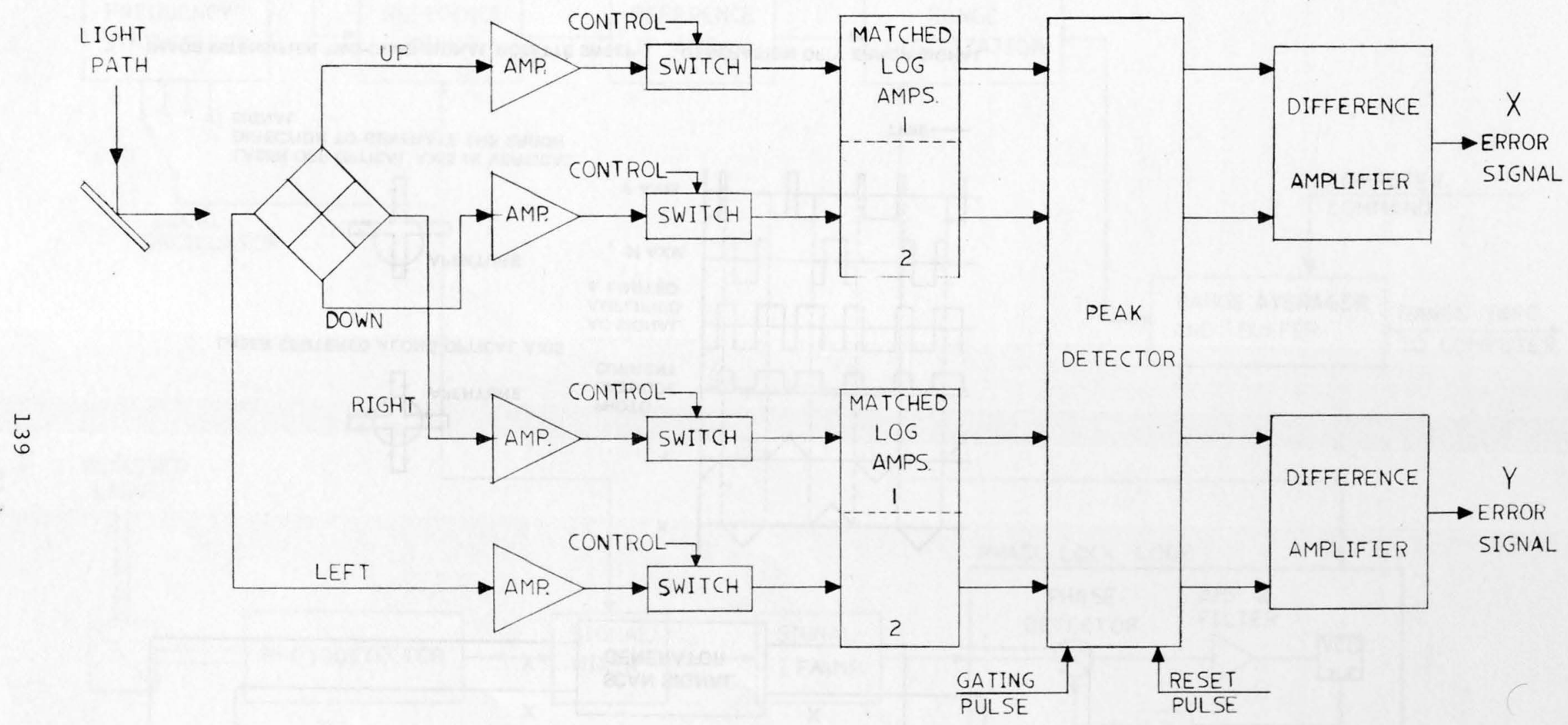

Fig, 4.2-4. Four-quadrant tracking receiver 


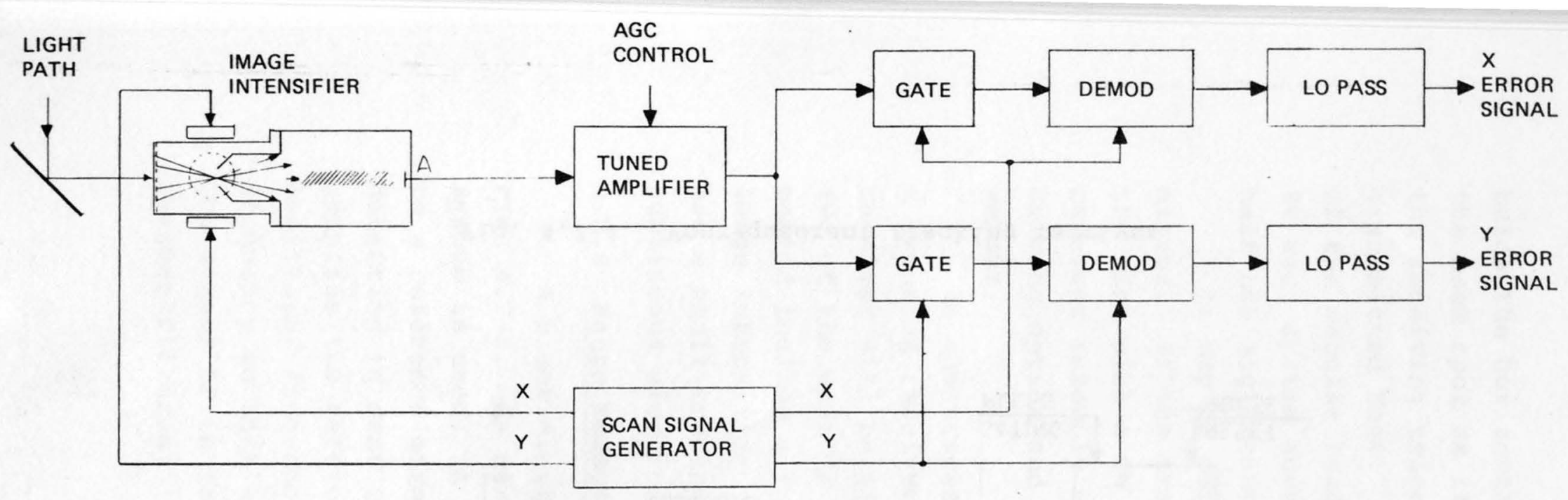

$\stackrel{\leftrightarrow}{\stackrel{\leftrightarrow}{\circ}}$

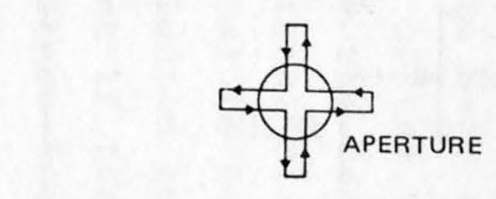

LASER CENTERED ALONG OPTICAL AXIS

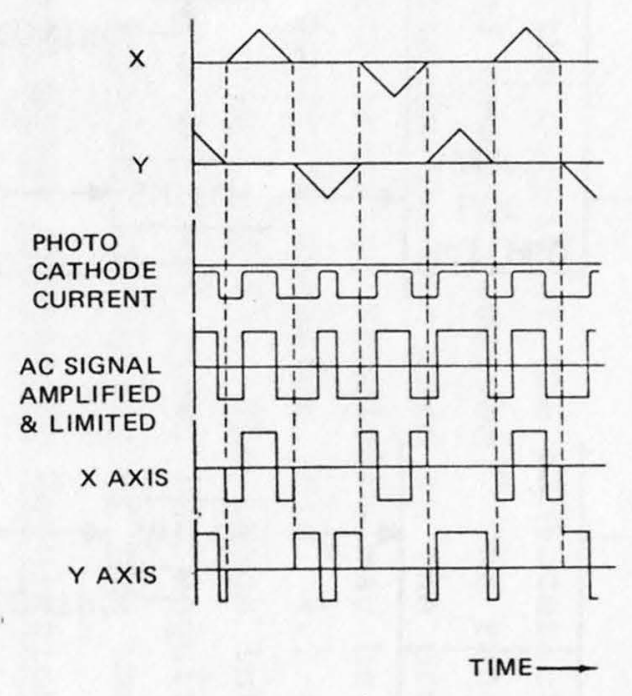

IMAGE INTENSIFIER TWO-DIMENSIONAL ROSETTE SWEEP

GENERATION OF Y ERROR SIGNAL

Fig. 4.2-5. Image intensifier tracking receiver 


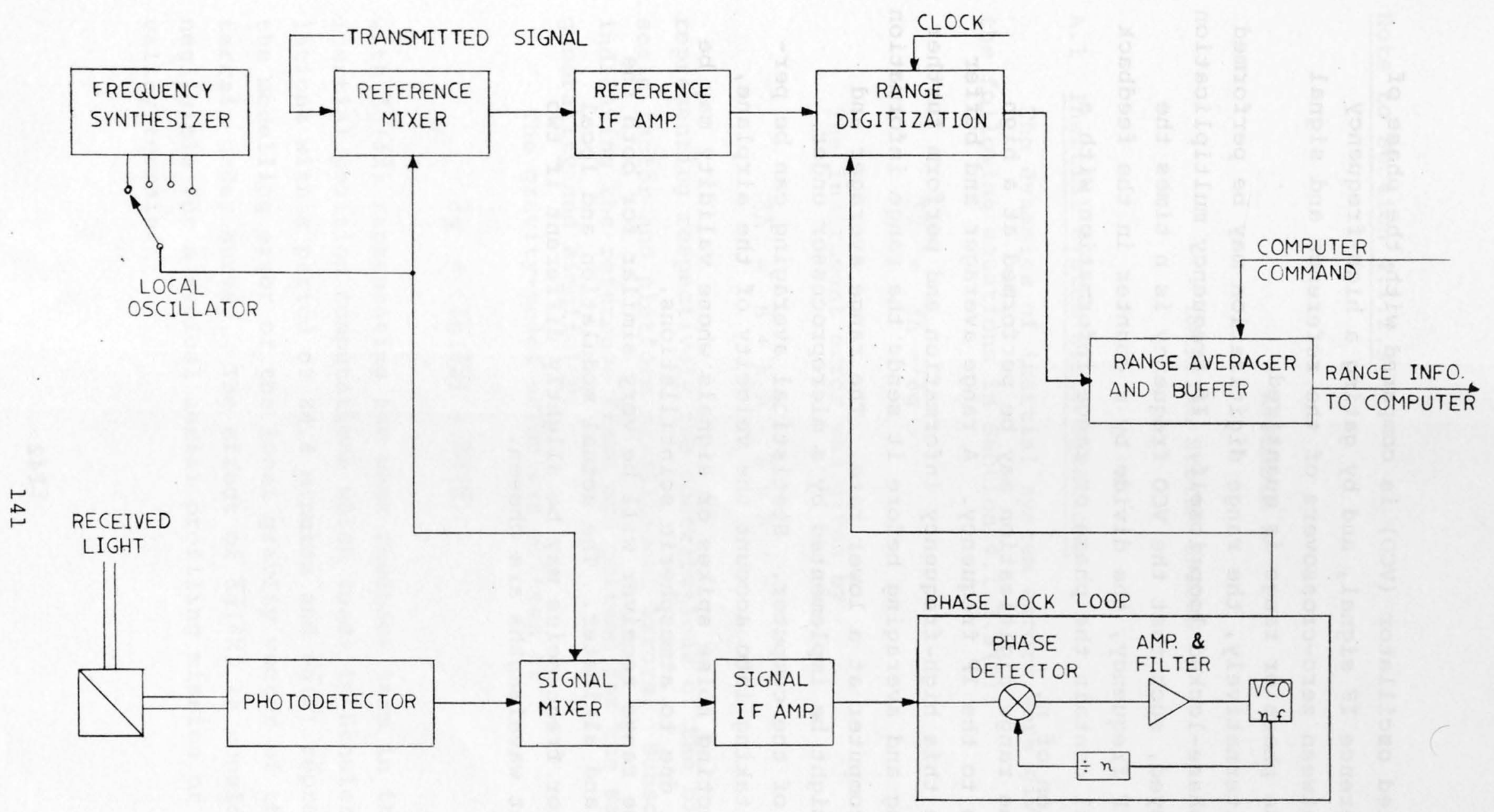

Fig. 4.2-6, Ranging Receiver 
controlled oscillator ( $\mathrm{VCO}$ ) is compared with the phase of the reference IF signal, and by gating a high-frequency clock between zero-crossovers of the reference and signal IF's, the phase or range is quantized.

Alternatively, the range digitization may be performed by the phase-locked loop itself. If frequency multiplication is employed, such that the VCO frequency is $\mathrm{n}$ times the signal IF frequency, the divide by $\mathrm{n}$ counter in the feedback loop will contain the phase or range information with a resolution of $n$,

The range digitization may be performed at a high rate, up to the IF frequency. A range averager and buffer can take this high-frequency information and perform further smoothing and averaging before it sends the range information to the computer at a lower rate. The range averager and buffer might be implemented by a microprocessor under control of the computer. Statistical averaging can be performed, taking into account the velocity of the airplane, and rejecting noise spikes or signals whose validity may be doubtful due to atmospheric scintillations.

The range receiver will be very similar for both the tracker and altimeter. The actual modulation and local oscillator frequencies may be slightly different if two different wavelengths are chosen. 
Note to Section 4.1 .2 .3

APPENDIX A

INERTIAL SYSTEM ERRORS

\section{A.1 Inertial System Error Equations}

The dynamics of inertial system errors were given by the following equations in Section $4.1 .2 .3(\mathrm{a})$ :

$$
\overline{\delta \ddot{X}}=\overline{\delta \bar{A}}+\overline{\delta g}
$$

The instrument error was given by

$$
\overline{\delta \bar{A}}=\bar{B}+k_{a} \bar{A}-\bar{\psi} \times \bar{A}
$$

representing respectively the contributions of the bias, scale factor and platform misalignment errors. Subscripts indicating the reference frame are omitted for the sake of generality and simplicity.

The gravity-model error was written as

$$
\overline{\delta g}=\overline{\delta g}(\overline{\delta x})+\overline{\delta g}(\bar{x})
$$

with $\overline{\delta g}(\overline{\delta x})$ representing the weak feedback term in the inertial position computations which leads to Schuler oscillations with a period of 84.4 minutes and $\overline{\delta g}(\bar{X})$ representing the modelling error of the local gravity vector of the terrain under survey. The effect of $\overline{\delta g}(\overline{\delta X})$ is considered negligible for a typical aerial profiling mission of rivervalley terrain. 


\section{A. 2 Growth Patterns for a General Path}

We shall consider the inertial system error-growth pattern for a general flight path as shown in Fig. A-l.

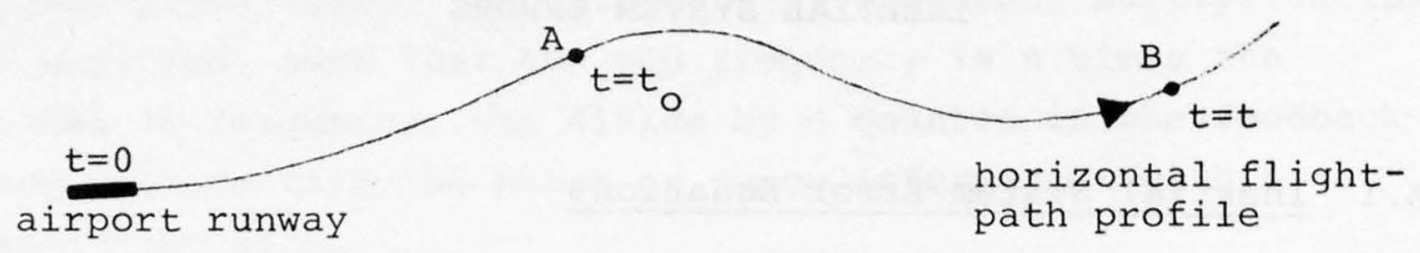

Fig. A-1. Flight-path profile

The growth patterns due to instrument and gravitymodel errors are generally functions of time, the initial and final coordinates of the flight path, and the geometry of the flight path as well, It is important to know the functional dependence of the growth patterns on the above parameters before an attempt can be made to isolate individual components from the total error and estimate the instrument and gravity-model biases from the patterns so isolated. Also, knowledge of the functional dependence of the error growth on the time, pattern of the flight path, etc., helps in defining the various system elements.

The flight path is given by

$$
\ddot{\overline{\mathrm{X}}}=\overline{\mathrm{A}}+\overline{\mathrm{g}}
$$

where $\bar{g}=\bar{g}_{0}+\overline{\delta g}, \bar{g}_{0}=$ gravitational acceleration from gravity model. 
A.3 Effect of Platform Misalignment, Accelerometer Bias and Gravity-Model Bias Errors

We shall consider the effect of platform misalignment $\bar{\psi}_{0}$ at $t=t_{0}$ on the inertial system error growth for $t>t_{0}$. The error growth due to platform misalignment and accelerometer bias is given by

$$
\begin{aligned}
\ddot{\delta \bar{X}} & =-\bar{\psi}_{0} \times \overline{\mathrm{A}}+\overline{\mathrm{B}} \\
\dot{\delta \bar{X}}\left(t_{0}\right) & =\dot{\delta \dot{X}}_{0} \text { given } \\
\overline{\delta \bar{X}}\left(t_{0}\right) & =\overline{\delta x}_{0} \text { given }
\end{aligned}
$$

Using $(A-1)$ in $(A-2)$ we get

$$
\ddot{\delta \bar{x}}=-\bar{\psi}_{0} \times \ddot{\bar{x}}+\bar{\psi}_{0} \times \bar{g}+\bar{B}
$$

We shall consider the effects of vehicle acceleration, gravity acceleration and bias separately.

A. 4 Vehicle Acceleration, $\ddot{\overline{\mathrm{X}}}$

Consider

$$
\ddot{\bar{\delta}}=-\bar{\psi}_{0} \times \ddot{\bar{x}}
$$

Integrating $(A-5)$ and using the initial conditions from $(A-3)$, we get

$$
\dot{\delta \dot{x}}=\frac{\dot{\bar{x}}_{0}}{-\bar{\psi}_{0}} \times\left[\dot{\bar{x}}_{-} \dot{\bar{x}}_{0}\right]
$$

and

$$
\overline{\delta x}=\overline{\delta x}_{0}+\left[\dot{\delta \dot{x}}_{0}+\bar{\psi}_{0} x \dot{\bar{x}}_{0}\right]\left[t-t_{0}\right]-\bar{\psi}_{0} x\left[\bar{x}-\bar{x}_{0}\right]
$$




\section{A. 5 Gravity Acceleration and Bias}

Consider

$$
\frac{\ddot{\delta}}{\mathrm{X}}=\bar{\psi}_{\mathrm{O}} \times \overline{\mathrm{g}}+\overline{\mathrm{B}}_{\mathrm{H}}+\overline{\mathrm{B}}_{\mathrm{Z}}
$$

where $\overline{\mathrm{B}}_{\mathrm{H}}$ represents the bias vector for horizontal accelerometers and $\bar{B}_{Z}$ represents the bias vector for the vertical accelerometer. For an initial level alignment of the stable platform of the IMU, on the airport runway, by nulling the output of horizontal accelerometers, we have

$$
\overline{\mathrm{B}}_{\mathrm{H}}+\bar{\psi}_{\mathrm{O}} \times \overline{\mathrm{g}}_{\mathrm{O}}=0
$$

where

time $t_{0}=0$.

$\overline{\mathrm{B}}_{\mathrm{H}_{\mathrm{O}}}$ and $\overline{\mathrm{g}}_{\mathrm{O}}$ represent the values of $\overline{\mathrm{B}}_{\mathrm{H}}$ and $\overline{\mathrm{g}}$ at

Combining $(A-8)$ and $(A-9)$, we get

$$
\delta \ddot{\bar{x}}=\bar{\psi}_{O} \times \Delta \bar{g}+\Delta \bar{B}_{H}+\bar{B}_{Z}
$$

where

and

$$
\Delta \bar{g}=\bar{g}-\bar{g}_{0}
$$

$$
\Delta \overline{\mathrm{B}}_{\mathrm{H}}=\overline{\mathrm{B}}_{\mathrm{H}}-\overline{\mathrm{B}}_{\mathrm{H}}
$$

\section{A. 6 Solution}

Considering only the non-changing components of $\Delta \bar{g}$, $\Delta \overline{\mathrm{B}}_{\mathrm{H}}$ and $\overline{\mathrm{B}}_{\mathrm{Z}}$ over the time period $\left(t_{0}, t\right)$, and combining the solutions of $(A-10)$ with $(A-5)$, we get: 


$$
\begin{aligned}
& \delta \dot{\bar{x}}=\delta \dot{\mathrm{x}}_{0}-\bar{\psi}_{0} \mathrm{x}\left(\dot{\overline{\mathrm{x}}}-\dot{\overline{\mathrm{x}}}_{0}\right)+\Delta \overline{\mathrm{g}} t+\overline{\mathrm{B}} t \quad(\mathrm{~A}-12) \\
& \delta \overline{\mathrm{x}}=\delta \overline{\mathrm{x}}_{0}+\left(\delta \dot{\overline{\mathrm{x}}}_{0}+\bar{\psi}_{0} \mathrm{x} \dot{\overline{\mathrm{x}}}_{0}\right)\left(t-t_{0}\right) \\
& -\bar{\psi}_{0} x\left(\bar{x}-\bar{x}_{0}\right)+\frac{1}{2} \Delta \bar{g} t^{2}+\frac{1}{2} \bar{B} t^{2} \quad(A-13)
\end{aligned}
$$

where, for the sake of simplicity, from here on we shall let $\overline{\mathrm{B}}$ represent $\Delta \overline{\mathrm{B}}_{\mathrm{H}}+\overline{\mathrm{B}}_{Z}$ rather than $\overline{\mathrm{B}}_{H}+\overline{\mathrm{B}}_{Z}$.

\section{A. 7 Special Cases}

For the sake of simplicity and brevity, we shall limit the discussion below to the effect of initial platform misalignment, omitting the consideration of the effect of accelerometer biases, .

Case 1. $\quad t_{0}=0$

In this case, $\bar{x}=\dot{\bar{x}}_{0}=\Delta \bar{g}=\Delta \bar{B}_{H}=0$. It may be remembered that we are including only the non-changing components of $\Delta \bar{g}$ and $\Delta \overline{\mathrm{B}}_{\mathrm{H}}$ and thus $\Delta \overline{\mathrm{g}}$ and $\Delta \overline{\mathrm{B}}_{\mathrm{H}}$ are zero by definition (See Eq. $A-1)$.

Thus,

$$
\dot{\delta \dot{x}}=\delta \dot{\bar{x}}_{0}-\bar{\psi}_{0} \mathrm{x} \dot{\overline{\mathrm{x}}}
$$

and

$$
\delta \overline{\mathrm{x}}=\delta \overline{\mathrm{x}}_{0}+t \dot{\delta}_{0}-\bar{\psi}_{0} \mathrm{x} \overline{\mathrm{x}}
$$

Thus, the components $\bar{\psi}_{0} \mathrm{x} \dot{\overline{\mathrm{x}}}$ and $\bar{\psi}_{\mathrm{O}} \mathrm{x} \overline{\mathrm{x}}$, of the vehicle velocity and the position in the misaligned frame, represent the effect of initial misalignment $\bar{\psi}_{0}$. The position and velocity errors at any given instant are thus a function of only the current vehicle position and velocity and not the flight path history or the time spent on the flight path. The $x, y, z$ components of the position error are given 
by

$$
\left[\begin{array}{l}
\delta x \\
\delta y \\
\delta z
\end{array}\right]=\left[\begin{array}{c}
\psi_{z}{ }^{r} \\
-\psi_{z}{ }_{x} \\
-\psi_{x}{ }^{r}+\psi_{y}{ }^{r}
\end{array}\right]+\left[\begin{array}{c}
-\psi_{y} \\
+\psi_{x} \\
0
\end{array}\right]{ }_{z}
$$

where $\bar{x}=\left[\begin{array}{l}r_{x} \\ r y \\ r \\ z\end{array}\right]$

It may be noted that the most significant contribution to the error growth is due to the horizontal components of the position vector $\bar{x}$. The effect of the vertical component of the position vector is very small because of the limitations on the altitude of the flight path imposed by various considerations discussed in Section 4.1-4.

Case 2. Straight-Line Level Path During $(t, t)$

In this case,

$$
\stackrel{\dot{\mathrm{X}}}{2 \text { constant }}
$$

and

$$
\bar{x}-\bar{x}_{0}-\dot{\bar{x}}_{0}\left(t-t_{0}\right) \text { represents the deviations of }
$$

the flight path from a straight-line flight path due to wind gusts, pilot error, etc.

Thus, from Equations $(A-11)$ and $(A-12)$, it may be seen that

$$
\dot{\delta \dot{x}} \simeq \dot{\delta x}_{0}
$$

and

$$
\overline{\delta \bar{x}} \simeq \frac{\dot{\delta x}_{0}}{\left(t-t_{0}\right)}
$$




\section{A. 8 The Effect of Gyro Drift Rate, $\bar{\varepsilon}$}

The IMU drift growth due to the gyro drift rate, $\bar{\varepsilon}$, is given by

$$
\begin{aligned}
\delta \ddot{\bar{X}} & =-t \bar{\varepsilon} \times \bar{A} \\
& =-t \bar{\varepsilon} \times[\ddot{\ddot{X}}-g]
\end{aligned}
$$

using E'q. $(A-1)$,

We shall consider the effect of vehicle acceleration and gravity separately.

A.9 Vehicle Acceleration, $\ddot{\bar{x}}$

Consider

$$
\delta \ddot{\ddot{\mathrm{x}}}=-t \bar{\varepsilon} \cdot \mathrm{x}
$$

Integrating $(A-22)$ and using the initial conditions from $(4.1-6)$, we get

$$
\begin{aligned}
\delta \overline{\mathrm{x}}= & \delta \overline{\mathrm{x}}_{0}+\delta \dot{\overline{\mathrm{x}}}_{0} \Delta t+\overline{\widetilde{\psi}}_{0} \times\left[\dot{\overline{\mathrm{x}}}_{0}+\overline{\widetilde{v}}\left(t, t_{0}\right)\right] \Delta t \\
& -\Delta \overline{\tilde{\psi}} \times \overline{\widetilde{R}}\left(t, t_{0}\right)
\end{aligned}
$$

and

$$
\delta \dot{\bar{x}}=\delta \dot{\bar{x}}_{0}-\overrightarrow{\widetilde{\psi}}_{0} \times \dot{\Delta \bar{x}}-\overrightarrow{\Delta \widetilde{\psi}} \times\left[\dot{\bar{x}}-\overline{\widetilde{v}}\left(t, t_{0}\right)\right] \quad(A-24)
$$

where

$$
\begin{aligned}
& \Delta t=t-t_{0} \\
& \overline{\widetilde{\psi}}_{0}=t_{0} \bar{\varepsilon} \text {, } \\
& \overline{\Delta \widetilde{\psi}}=\Delta t \bar{\varepsilon}, \\
& \overline{\widetilde{R}}\left(t, t_{0}\right)=\left(\bar{x}-\bar{x}_{m}\right)+\left(\bar{x}_{0}-\bar{x}_{m}\right) \\
& \bar{x}_{m}=\frac{1}{\Delta t} \int_{t}^{t} \bar{x} d t \\
& \Delta \dot{\overline{\mathrm{x}}}=\dot{\overline{\mathrm{x}}}-\dot{\overline{\mathrm{x}}}_{\mathrm{O}}^{\mathrm{O}}
\end{aligned}
$$


and

$$
\overline{\tilde{V}}\left(t, t_{0}\right)=\frac{\Delta \dot{\bar{X}}}{\Delta t}
$$

A tilde $(\sim)$ has been placed over symbols previously used to create more symbols,

We shall simplify Equations $(A-23)$ and $(A-24)$ further by considering the following two cases:

Case 1: $\quad t_{0}=0$, i.e., total error growth

Case 2: $t_{0} \neq 0$, i.e., incremental error growth since $t_{0}$.

\section{Case 1. $\underline{t}_{0}=0$ at Take-off From Airport Runway}

In this case

$$
\dot{\mathrm{x}}_{0}=\overline{\widetilde{\psi}}_{0}=0
$$

Further, let

$$
\delta \overline{\mathrm{X}}(t=0)=\delta \dot{\overline{\mathrm{X}}}(t=0)=0
$$

for simplicity. From $(A-23)$ and $(A-24)$, we get

$$
\delta \dot{\overline{\mathrm{X}}}=-t \bar{\varepsilon} \times \overline{\widetilde{R}}
$$

and

$$
\delta \dot{\bar{X}}=-\bar{\varepsilon} \times[t \dot{\bar{X}}-(\bar{X}-\bar{x}(t=0))]
$$

Equations $(A-28)$ and $(A-29)$ represent the total growth in the inertial system position and velocity error since the time of take-off from the airport runway.

\section{Case 2. $\quad \underline{t}_{0} \neq 0$}

Here we are interested only in the incremental growth in the system error since time $t=t_{0}$. 
Evaluating $(A-28)$ and $(A-29)$ at times $t=t_{0}$ and $t=t$, we get after some manipulation the following expressions for the inertial system error growth.

$$
\delta \dot{\overline{\mathrm{X}}}=\delta \dot{\overline{\mathrm{x}}}_{0}-\overline{\tilde{\psi}}_{0} \mathrm{x}\left[\dot{\overline{\mathrm{X}}}-\dot{\overline{\mathrm{X}}}_{0}\right]-\Delta \overline{\widetilde{\psi}} \times\left[\dot{\overline{\mathrm{X}}}-\overline{\overrightarrow{\mathrm{V}}}\left(t, t_{0}\right)\right] \quad(\mathrm{A}-30)
$$

and $\delta \overline{\mathrm{x}}=\delta \overline{\mathrm{x}}_{0}-\overline{\widetilde{\psi}}_{0} \mathrm{x}\left[\overline{\mathrm{x}}-\overline{\mathrm{x}}_{0}\right]-\Delta \overline{\tilde{\psi}} \times \overline{\widetilde{R}}\left(t, t_{0}\right)$

where

$$
\begin{aligned}
& \delta \overline{\mathrm{x}}=-\overline{\widetilde{\psi}}_{0} \times \overrightarrow{\widetilde{R}}\left(t_{0}, 0\right) \\
& \delta \overline{\mathrm{x}}_{0}=-\overline{\tilde{\psi}}_{0} \times\left[\dot{\bar{x}}_{0}-\overline{\widetilde{V}}\left(t_{0}, 0\right)\right]
\end{aligned}
$$

and $\overline{\widetilde{\psi}}_{0}$ and $\Delta \overline{\widetilde{\psi}}$ are as dẹfined in $(\mathrm{A}-25)$.

The effect of the $\overline{\tilde{\psi}_{0}}$ terms in the expressions for $\delta \dot{\bar{x}}$ and $\delta \overline{\mathrm{x}}$ derived above is similar to the effect of platform initial misalignment considered earlier. The $\Delta \bar{\psi}$ terms also could be given a simple geometrical interpretation as follows.

From the definition of $\overline{\tilde{V}}\left(t, t_{0}\right)$, it may be seen that $\overline{\widetilde{V}}\left(t, t_{0}\right)$ represents the mean velocity of the aircraft during the interval $\left(t, t_{0}\right)$. Thus, $\dot{\bar{X}}-\overline{\widetilde{V}}\left(t, t_{0}\right)$ represents at $t$ ime $t$ the deviation of the velocity of the aircraft from the mean. Thus, for an unaccelerated flight segment such as a straightline constantraltitude flight, there is no velocity error growth due to platform angular misalignment rate.

The significance of the $\tilde{R}\left(t, t_{0}\right)$ term in the position error growth expression can be easily understood from the flight path diagram of Figure $\mathrm{A}-2$. 


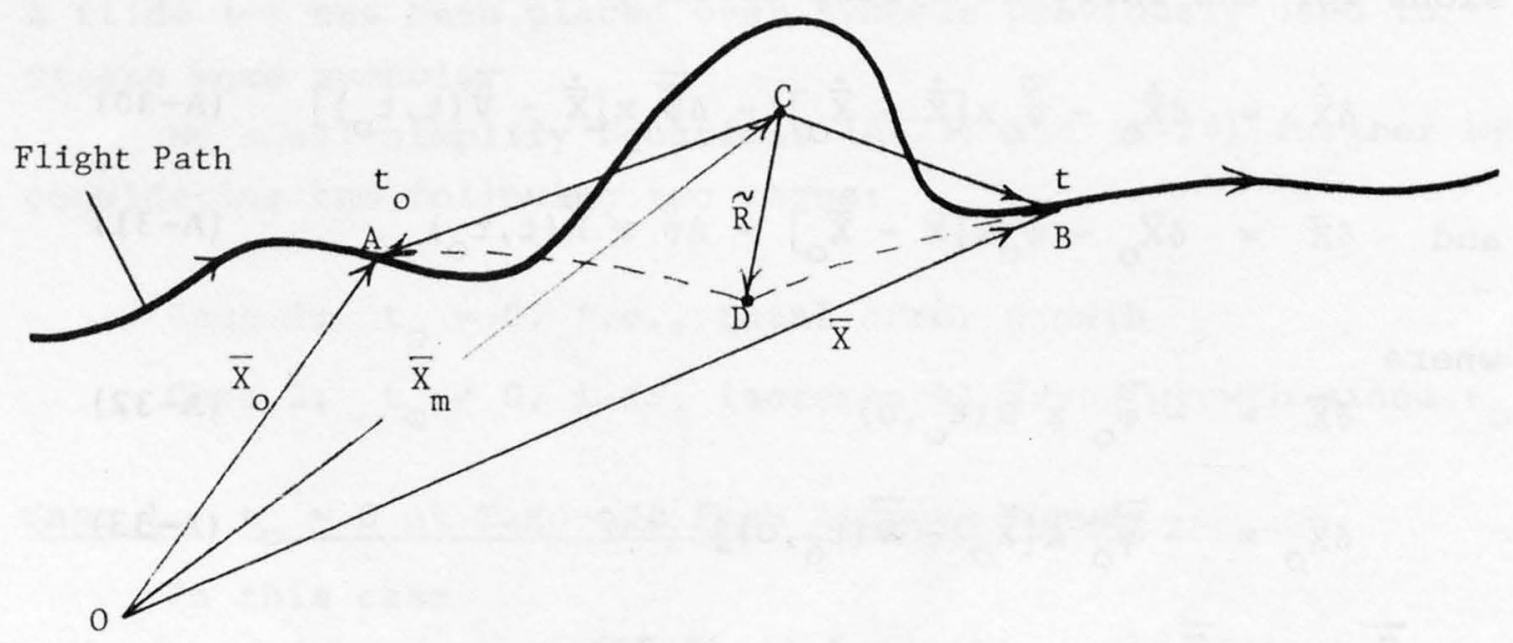

Earth-fixed Reference Frame

Fig. A-2, Flight-path diagram

Vectors $O A$ and $O B$ represent the position vectors $\bar{x}_{0}$ and $\bar{x}$ of the aircraft at times $t_{0}$ and $t$, respectively, with respect to the reference frame located at 0 . Vector oC represents the mean position of the aircraft during the time interval $\left(t_{0}, t\right)$. Thus, vector $C D$, which represents the vector sum of deviations from the mean of aircraft position at the beginning and end of the time interval, represents $\vec{R}$ (See Eq. $(A-25)$ ). The vector product of $\Delta \overline{\widetilde{\psi}}$ (i.e., $\Delta t \bar{\varepsilon}$ ) with $\widetilde{\widetilde{R}}$ represents one of the components of the inertial system error growth, the other component being given by the vector product of $\overline{\widetilde{\psi}}_{0}\left(i . e, t_{0} \bar{\varepsilon}\right)$ with vector $A B$.

For non-accelerating flight paths, the latter component is the more dominant source of error in the inertially indicated position.

A few observations can be made from the above 
concerning the error growth due to $\Delta \tilde{\psi}$ for a typical aerial survey flight path of Figure A-2:

(1) It does matter what path is taken from point $A$ to point $B$ in the case of the platform angular drift rate as contrasted with the case of initial platform angular misalignment.

(2) For a straight-line unaccelerated flight path between any two points on the flight path, $\overrightarrow{\widetilde{R}}$ and $\overrightarrow{\widetilde{V}}$ are both zero.

(3) Deviations from a straight-line flight path arise due to planned turns and unplanned deviations primarily due to the effect of wind gusts and the aircraft flight path control (autopilot or pilot) error. In the case of turns, it may be easily seen that the tighter the turn, the smaller the error. In the case of flight path deviations due to wind gusts, the rms is given by

$$
\sigma_{\Delta \bar{X}} \simeq 2 \Delta \bar{\psi} \times \bar{\sigma}_{R}
$$

where $\bar{\sigma}_{\tilde{R}}$ represents the rms flight path deviations. (4) In the horizontal plane the position error is mainly an along-track error.

We have considered in the above the inertial system error growth due to the coupling of vehicle accelerations and the platform angular drift rate. We shall consider next the most significant coupling of the drift rate with the effect of gravity.

\section{A.10 Gravity Acceleration}

In this case, the inertial system error growth is given by

$$
\delta \dot{\overline{\mathrm{X}}}=t(\bar{\varepsilon} \times \overline{\mathrm{g}})
$$


The effect of the gravity vector differs from the effect of vehicle acceleration vector due to the significant differences in the magnitude and directional characteristics of the two vectors. The magnitude of the vehicle acceleration vector is significantly smaller than the magnitude of the gravity vector during the most significant portion of the flight path, namely, the surveying runs. Secondly, the magnitude of the gravity vector and the direction of the vector are unchanging at all times except for minor deviations over different parts of the terrain, Thus, for a given $\bar{\varepsilon}$, the error growth is merely a function of the time spent on the flight path and not a function of the geometry of the flight path and is given by

$$
\delta \bar{x}=\frac{t^{3}}{6} \bar{\varepsilon} \times \bar{g}
$$

which represents a solution of $(\mathrm{A}-35)$ with zero initial conditions. The effect of gravity vector variations is negligible, the percentage deviations from the nominal value being very small.

Thus, the coupling of the gravity vector with the gyro drift rate vector produces significantly large contributions to $\Delta \overline{\mathrm{X}}$ and the error growth is proportional to $t^{3}$.

Since $\bar{g}$ is a vertical vector, $\bar{\varepsilon} \times \bar{g}$ has components in the horizontal plane only. Thus, the gravity coupling is of importance for the error growth in the horizontal channels of the inertial system.

\section{A.11 The Effect of Gravity Model Errors}

From (4.1-6) and (4.1-8) the inertial system error growth due to gravity model errors is given by

$$
\delta \dot{\overline{\mathrm{x}}}=\overline{\delta g}(\overline{\mathrm{X}})
$$


The effect of the gravity feedback term $\delta \bar{g}(\delta \bar{x})$ is considered negligible as explained earlier.

As in the case of vehicle accelerations considered previously, the effect of gravity model errors on the inertial error growth depends upon the geometry of the flight-path segment due to the position dependence of $\delta \bar{g}$.

Let

$$
\delta \bar{g}=\left[\begin{array}{ll}
g_{0} & \delta \phi_{y} \\
g_{0} & \delta \phi_{x} \\
\delta g_{0}
\end{array}\right]
$$

In $(A-38)$ the components of $\bar{\delta}$ in the horizontal plane are characterized by $\delta \dot{\phi}_{\mathrm{Y}}$ and $\delta \phi_{\mathrm{X}}$ which represent the deflection angles of the gravity vector around the $\mathrm{x}$ and $\mathrm{y}$ axes, respectively,

Figures $A-3$ and $A-4$ represent the variations in the vertical deflection angles and $\delta g_{0}$ (termed anomaly) over a twenty-mile straight-line traverse across sample land areas near Denver and Des Moines, respectively. Anomaly is measured in milligals, where $1 \mathrm{gal}=1 \mathrm{~cm} / \mathrm{sec}^{2}$ and $1 \mathrm{mgal}=1 \times 10^{-3}$ $\mathrm{cm} / \mathrm{sec}^{2}$. The Denver case is representative of typical gravity model variations expected over most non-mountainous terrains, whereas the Des Moines case is indicative of one of the most severe spatial variations that could be expected. In both cases, it may be seen that the variations in the components across the 20-mile stretch are such that the spatial curvature of the variations is predominantly unidirectional. Thus, the variations could be approximated by a two- or three-segment straight-line model. If the line segments are appropriately. chosen, the deviations of the variations from the line segment model are positive as well as negative over the 20-mile 
ANOMALIES (mgal)

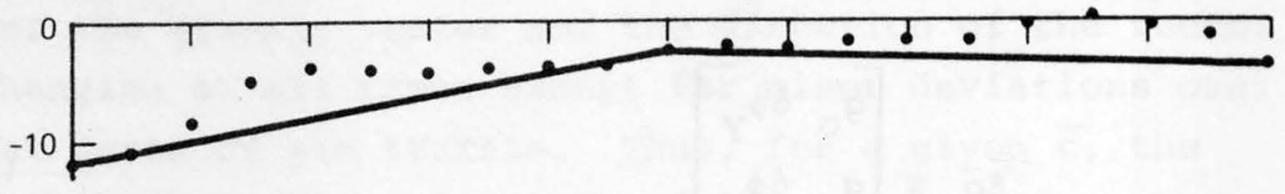

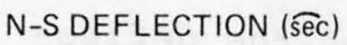

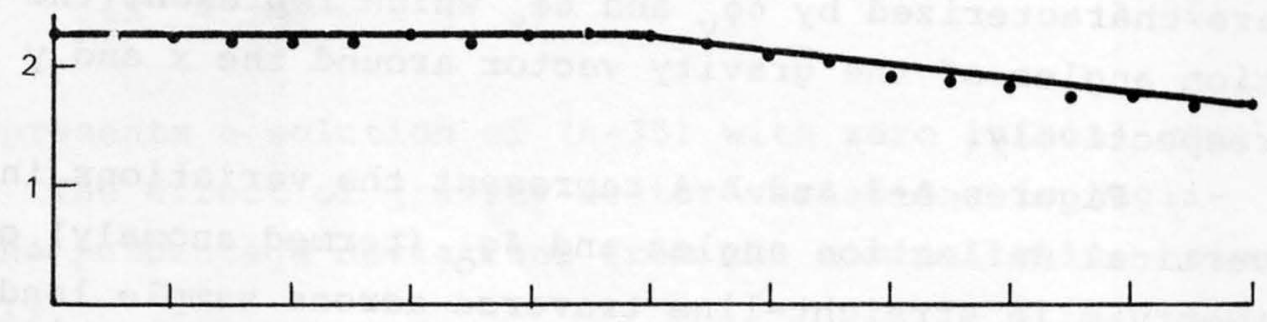

E-W DEFLECTION (sec)

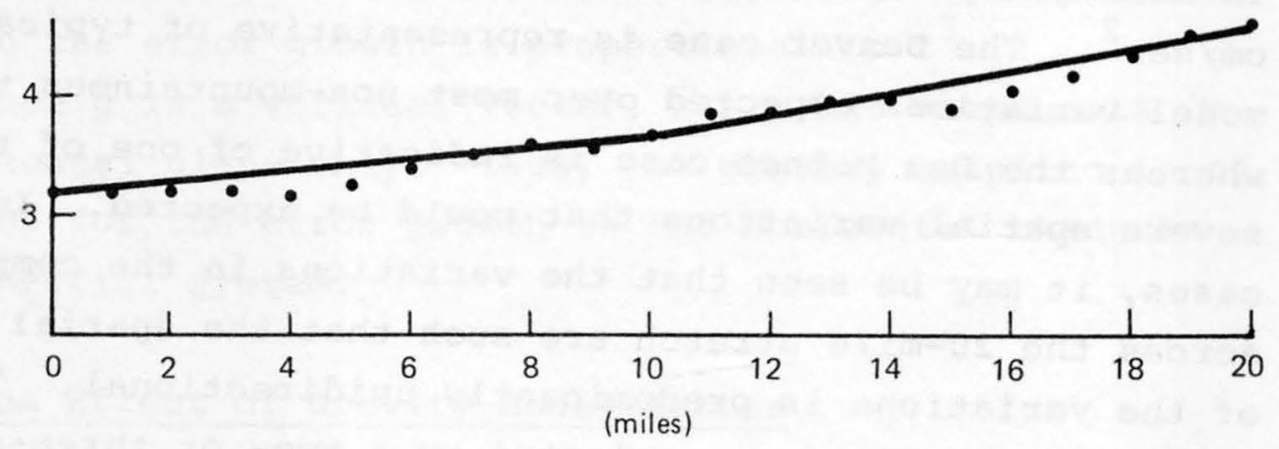

Fig. A-3. Typical case of gravity anomalies and deflections - Denver 
ANOMALIES (mgal)

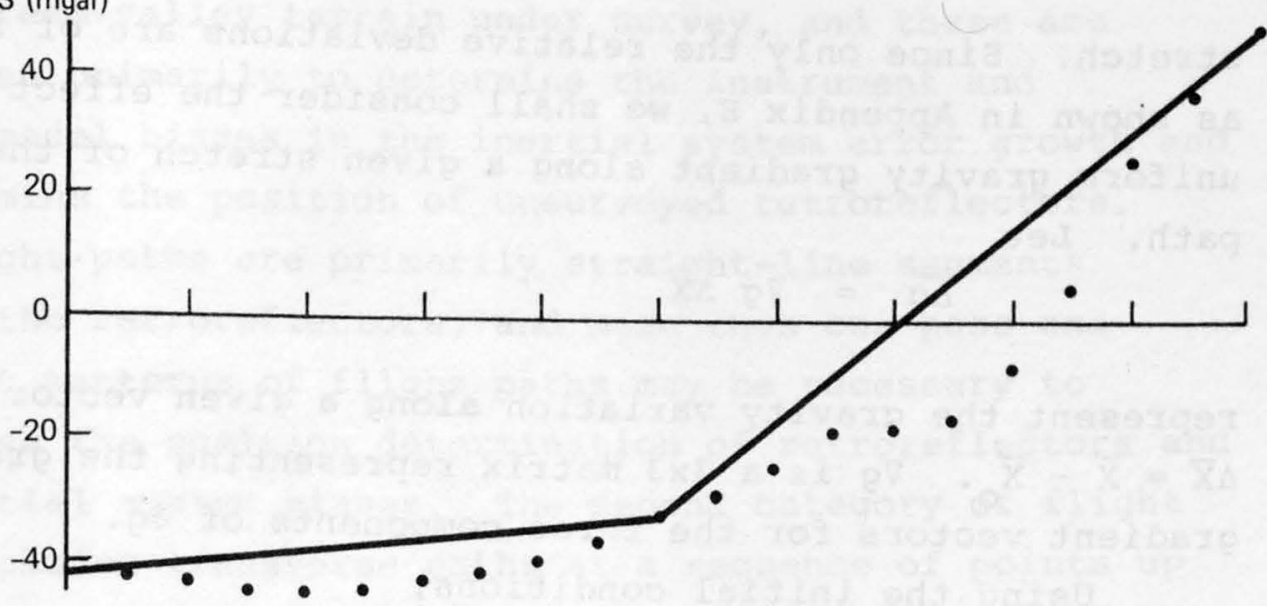

N-S DEFLECTION ( $\widehat{\mathrm{sec}})$

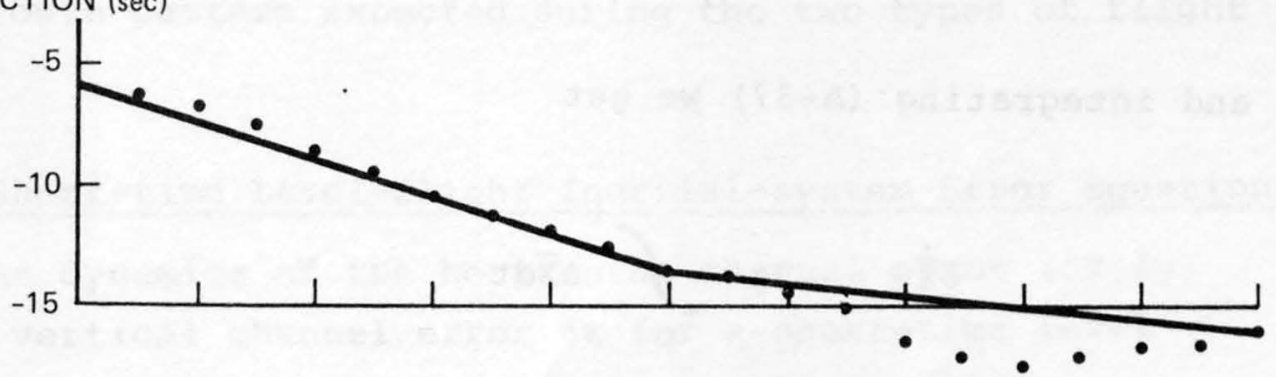

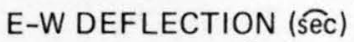

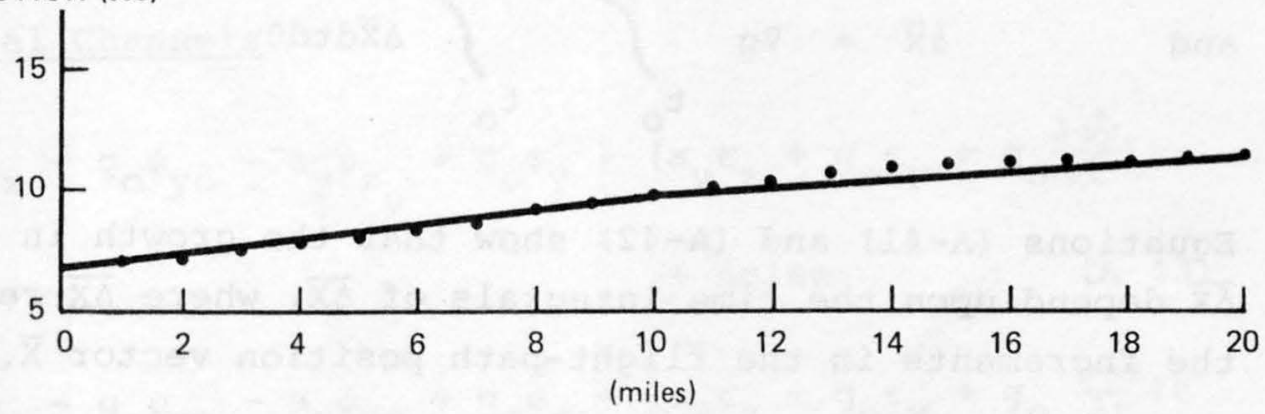

COMMENTS: (1) Gravity data assumed available only at 3 points - retroreflectors.

(2) Errors in the gravity model bias terms could be further reduced.

Fig. A-4. Worst case of gravity anomalies and deflections - Des Moines 
stretch. Since only the relative deviations are of interest as shown in Appendix $\mathrm{E}$, we shall consider the effect of a uniform gravity gradient along a given stretch of the flight path. Let

$$
\overline{\Delta g}=\nabla g \Delta \bar{x}
$$

represent the gravity variation along a given vector $\Delta \overline{\mathrm{x}}=\overline{\mathrm{x}}-\overline{\mathrm{x}}_{\mathrm{O}} \cdot \quad \nabla \mathrm{g}$ is a $3 \times 3$ matrix representing the gravity gradient vectors for the three components of $\delta \bar{g}$.

Using the initial conditions,

$$
\delta \overline{\mathrm{x}}_{0}=\delta \dot{\overline{\mathrm{x}}}_{0}=0
$$

and integrating $(A-37)$ we get

$$
\delta \dot{\bar{x}}=\nabla g \int_{t_{0}^{t}}^{t} \bar{x} d t
$$

and

$$
s \bar{x}=\nabla g \int_{t_{0}}^{t} \int_{t_{0}}^{\theta} \Delta \bar{x} d t d \theta
$$

Equations $(A-41)$ and $(A-42)$ show that the growth in $\dot{\delta \dot{X}}$ and $\overline{\delta X}$ depend upon the time integrals of $\overline{\Delta x}$, where $\overline{\Delta X}$ represents the increments in the flight-path position vector $\overline{\mathrm{x}}$.

\section{A.12 Error Growth Pattern for Aerial Survey Paths - Introduction}

The flight paths for an aerial survey mission have been classified in two categories. The first category is for flight paths that are generally along the full length of the 
local streammalley terrain under survey, and these are undertaken primarily to determine the instrument and gravity-model biases in the inertial system error growth and to determine the position of unsurveyed retroreflectors. Such flight paths are primarily straight-line segments between the retroreflectors, and more than one pass and different patterns of flight paths may be necessary to accomplish the position determination of retroreflectors and the inertial system biases. The second category of flight paths includes transverse paths at a sequence of points up the stream valley undertaken to determine the desired crosssection profiles. The following deals with the short-time error-growth pattern expected during the two types of flight paths.

\section{A.13 Short-time Level-flight Inertial-system Error Equations}

The dynamics of the horizontal channel error $(\delta x, \delta y)$ and the vertical channel error $\delta z$ for a short-time level flight are easily obtained from the equations below:

Horizontal Channels

$$
\begin{aligned}
& \delta \ddot{x}=b_{x}+g_{0} \psi_{y o}+a_{y} \psi_{0}+g_{0} \phi_{y}+\left(a_{y} \varepsilon_{z}+g_{0} \varepsilon_{y}+g_{0} \frac{\partial \phi \dot{y}}{\partial t}\right) t \\
& + \text { noise } \\
& (A-43) \\
& \delta \ddot{y}=b_{y}-g_{0} \psi_{x O}-a_{x} \psi_{z O}-g_{0} \phi_{x}-\left(a_{x} \varepsilon_{z}+g_{0} \varepsilon_{x}+g_{0} \frac{\partial \phi^{\prime} x}{\partial t}\right) t \\
& + \text { noise }
\end{aligned}
$$


Vertical Channels

$\delta \ddot{z}=b_{z}+a_{y} \psi_{x o}+a_{x} \psi_{y o}+\Delta g_{0}-\left(\varepsilon_{x} a_{y}+\varepsilon_{y} a_{x}+\frac{\partial \Delta g}{\partial t}\right) t$

+ noise

where $\delta \bar{X}=\left[\begin{array}{l}\delta x \\ \delta y \\ \delta z\end{array}\right], \quad \bar{B}=\left[\begin{array}{l}b_{x} \\ b_{y} \\ b_{z}\end{array}\right] \quad \bar{\psi}=\bar{\psi}_{x o}+t \bar{\varepsilon}$,

$$
\bar{\psi}_{0}=\left[\begin{array}{l}
\psi_{x O} \\
\psi_{y o} \\
\psi_{z o}
\end{array}\right] \quad \bar{\varepsilon}=\left[\begin{array}{l}
\varepsilon_{x} \\
\varepsilon_{y} \\
\varepsilon_{z}
\end{array}\right] \bar{A}=\left[\begin{array}{l}
a_{x} \\
a_{y} \\
a_{z}
\end{array}\right]
$$

$$
\overline{\delta g}=\left[\begin{array}{c}
g_{0} \phi_{y} \\
g_{0} \phi_{x} \\
\Delta g_{0}
\end{array}\right] \text { and }\left[\begin{array}{c}
\frac{\partial \phi_{x}^{\prime}}{\partial t} \\
\frac{\partial \phi_{\hat{y}}}{\partial t}
\end{array}\right]\left[\begin{array}{cc}
\frac{\partial \phi_{x}}{\partial x} & \frac{\partial \phi_{x}}{\partial y} \\
\frac{\partial \phi_{y}}{\partial x} & \frac{\partial \phi_{y}}{\partial y}
\end{array}\right]\left[\begin{array}{c}
v_{x} \\
v_{y}
\end{array}\right]
$$

in vector notation.

The initial conditions for the above equations represent the errors in the position and velocity fix. The dominant component in the velocity fix error is due to the misorientation of the stable platform "p" frame from the airborne geodetic reference "g" frame. The resulting errors in the velocity are given by 


$$
\begin{aligned}
& \dot{\delta x}(0)=v_{y} \psi_{z o} \\
& \dot{\delta y}(0)=-v_{x} \psi_{z o} \\
& \dot{\delta z}(0)=-v_{y} \psi_{x O}+v_{x} \psi_{y o}
\end{aligned}
$$

\section{A.14 Inertial System Error Growth for Straight-line Flight Paths}

From the above equations, the short-time model for the positive error growth $\delta x$ can be obtained easily. For example, the error growth of $\delta x$, the $x$ component of $\delta x$ for a straight-line unaccelerating flight path, can be easily seen to be given by

$$
\begin{gathered}
\delta x(t)=k_{0}+k_{1} t+k_{2} t^{2}+k_{3} t^{3}+\text { noise, } t<\frac{t_{s}}{10} \\
t_{s}=84 \text { mins. }
\end{gathered}
$$

where

$$
\begin{array}{ll}
\mathrm{K}_{0}=\delta \mathrm{x}(\mathrm{O}) & \begin{array}{l}
\text { (Position bias) } \\
\mathrm{K}_{1}=\psi_{\mathrm{z}} \mathrm{v}_{\mathrm{y}}
\end{array} \\
\mathrm{K}_{2}=\frac{1}{2}\left(\mathrm{~b}_{\mathrm{x}}+g_{0} \phi_{\mathrm{y}}\right) & \begin{array}{l}
\text { (Azimuth misalignment) } \\
\text { (Accelerometer bias and } \\
\text { gravity vector vertical } \\
\text { deflection) }
\end{array} \\
\mathrm{K}_{3}=\frac{90}{6}\left(\varepsilon_{y}+\frac{\partial \phi_{y}}{\partial x} v_{x}+\frac{\partial \phi_{y}}{\partial y} v_{y}\right) & \begin{array}{l}
\text { (Gyro drift rate } \\
\text { and gravity } \\
\text { vector vertical } \\
\text { deflection } \\
\text { gradient) }
\end{array}
\end{array}
$$

It may be seen that there are four patterns in the inertial system error growth. The effect of error in initial positionfix remains unchanged during the flight. The error due to azimuth misalignment in the orientation of the platform frame grows linearly with distance (and also time, in this case). 
The error due to accelerometer bias and gravity vector deflection grows in a quadratic fashion with time. The errors due to gyro drift rate (platform angular misalignment rate) and the gravity vector deflection gradient grow in a $t^{3}$ fashion. The steady error and the azimuth misalignment ( $t$ term) generally dominate the initial error growth to be, in turn, dominated by the $t^{2}$ and $t^{3}$ terms as time progresses. It may be commented that during a straight-line flight path, the horizontal accelerations are on the average zero. The effect of the high frequency deviations in the horizontal accelerations $a_{x}$ and $a_{y}$ on the error growth is very small, as shown earlier.

\section{A.15 Error Growth in The Inertially Indicated Position During Profiling}

We have discussed in previous sections the effect of instrument errors and gravity model errors on the inertially indicated position. The different components of the position error growth in the horizontal and vertical directions during profiling are shown in Figures $A-5$ and $A-6$. Figure $A-5 a$ shows a time tagged flight path and also defines the error components attributable to the longitudinal and lateral axes.

The nature of the different growth patterns shown in these figures is easily understood in the context of the detailed discussions in the previous sections. 
a)
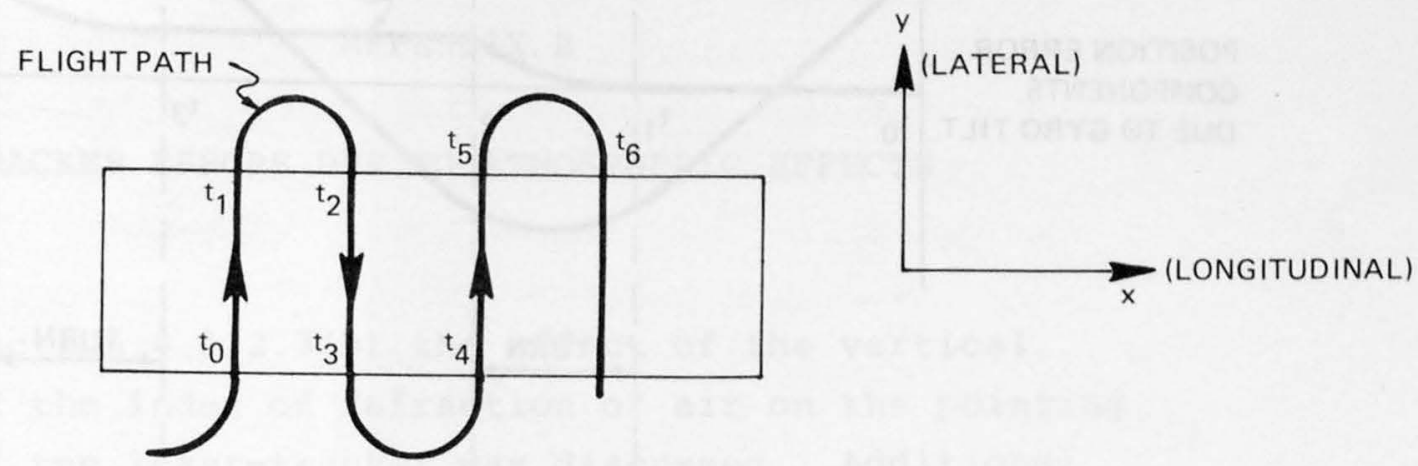

b)

POSITION ERROR COMPONENT DUE TO AZIMUTH ERROR

c)
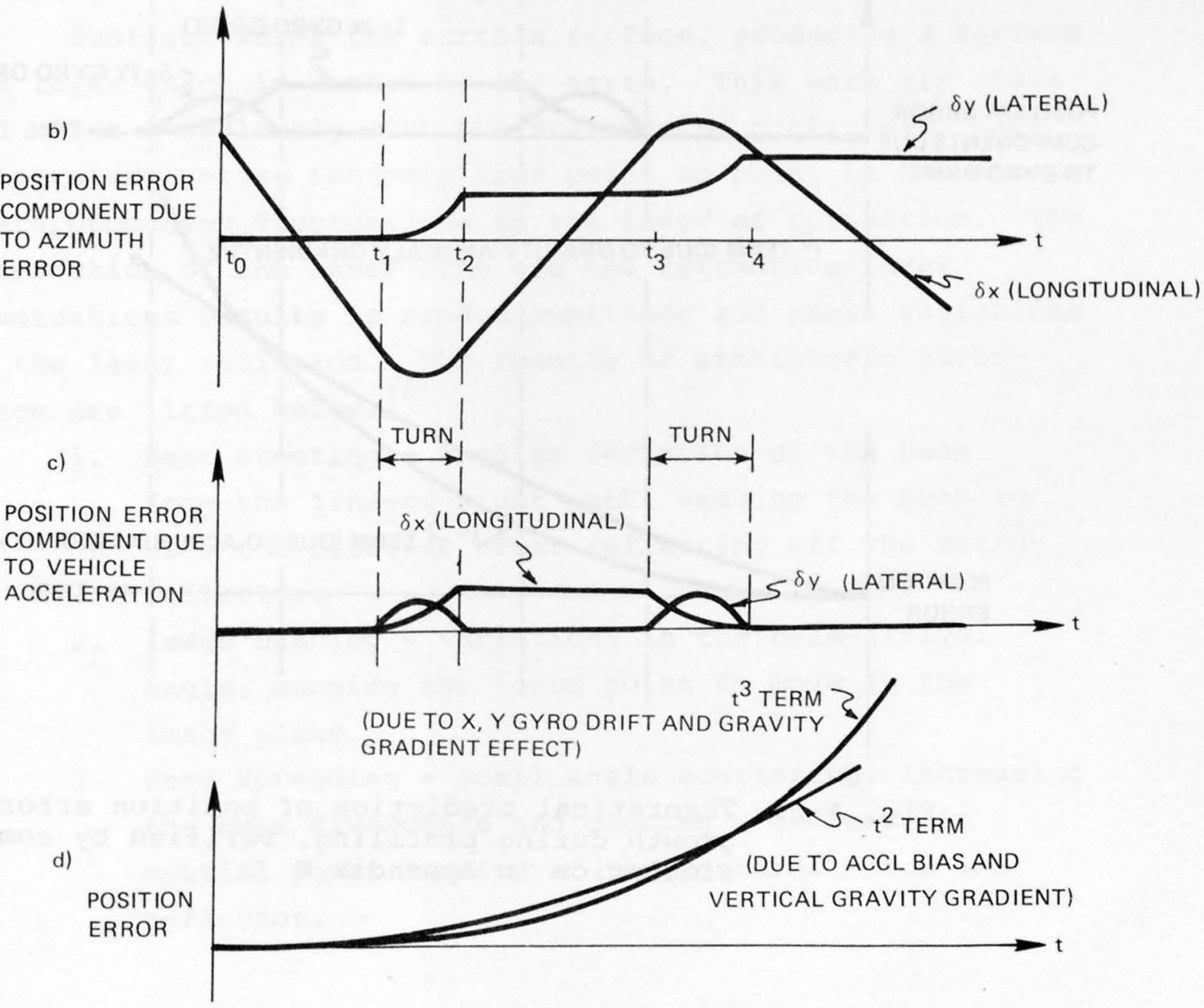

Fig. A-5. Theoretical prediction of lateral and longitudinal error growth during profiling, confirmed by computer simulation in Appendix E 


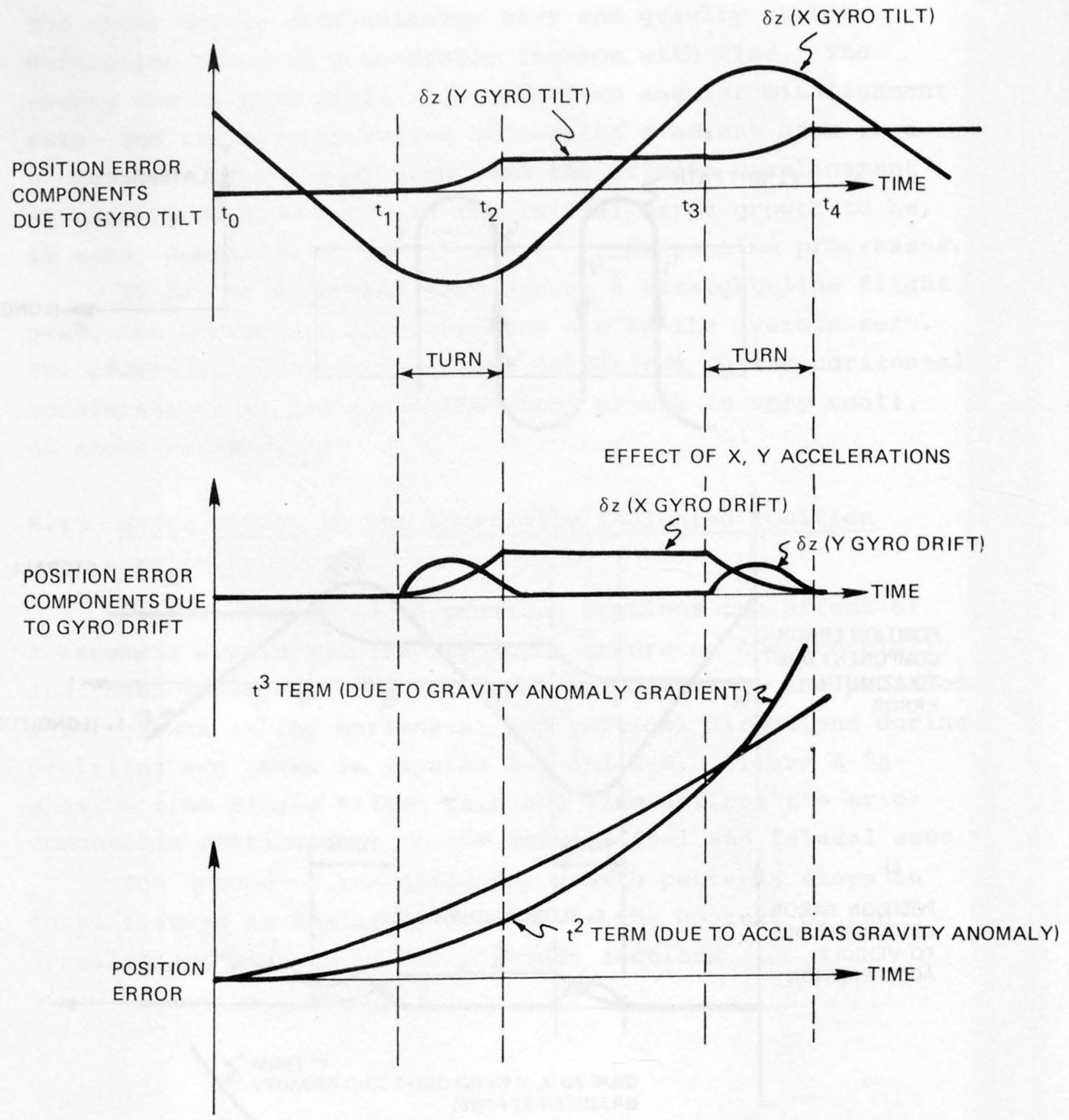

Fig, A-6, Theoretical prediction of position error growth during profiling, verified by computer simulation in Appendix $\mathrm{E}$ 
APPENDIX B

TRACKER ERRORS DUE TO ATMOSPHERIC EFFECTS

In Section 4.1.2.3(b) the effect of the vertical gradient of the index of refraction of air on the pointing accuracy of the laser-tracker was discussed. Additional pointing errors due to atmospheric turbulence can result.

Sunlight warms the earth's surface, producing a surface air layer which is heated by the earth. This warm air rises and mixes turbulently with the surrounding cooler air. Air temperature varies randomly from point to point in the atmosphere, producing fluctuations in the index of refraction. The interaction of the laser beam and the refractive index fluctuations results in random amplitude and phase variations of the laser radiation. The results of atmospheric turbulence are listed below: ${ }^{(8)}$

1. Beam Steering - angular deviation of the beam from the line-of-sight path, causing the beam to miss the receiver after reflecting off the retroreflector.

2. Image Dancing - variations in the beam-arrival angle, causing the focus point to move in the image plane.

3. Beam Spreading - small angle scattering, increasing the beam divergence and causing a decrease in spatial power density at the retroreflector and reflector. 
4. Beam Scintillation - small angle destructive interference within the beam cross section, causing variations in the spatial power density at the receiver.

5. Spatial Coherence Degradation - losses in phase coherence across the beam phase fronts, degrading the photomixing performance.

6. Polarization Fluctuations - variations in the laser beam polarization state.

In Reference 8 it is shown that the effects of beam steering are negligible when the laser beam diameter is much greater than the atmospheric inhomogeneity dimension as is the case for the laser tracker.

Image dancing results in a maximum r.m.s. deviation in laser beam arrival of about 3 microradians in intermediate atmospheric turbulence. This effect will average out over an observation time of a second or two and is also much less than the 100 microradian pointing error of the tracker (20 sec.).

Beam spreading and beam scintillation will result in a small decrease in the laser beam spatial power density and a small decrease in the signal-to-noise ratio of the lasertracker, respectively. The net effect of a decrease in signal-to-noise ratio is inconsequential due to the exceptionally large signal returning from a cooperative reflecting target.

Spatial coherence degradation and polarization fluctuations affect only heterodyne or homodyne optical detection systems. Simple detection of intensity modulation of the laser radiation remains unaffected. 
APPENDIX C

ESTIMATION OF' TERRAIN POSITION

\section{C.1 Introduction}

Figure $\mathrm{C}-1$ shows all the elements of the measurement geometry which obtain during aerial surveying in determining the position coordinates $\bar{x}_{\text {ter }}$ for the terrain under survey. This in turn requires the determination of the position of the aircraft.

The error-free measurement equations are:

$$
\begin{aligned}
& \overline{\mathrm{x}}_{\text {ter }}=\overline{\mathrm{x}}_{\mathrm{ac}}+\overline{\mathrm{H}}, \text { terrain survey equation }(\mathrm{C}-1) \\
& \overline{\mathrm{x}}_{\mathrm{ac}}=\overline{\mathrm{x}}_{\text {IMU }} \quad, \text { IMU equation }
\end{aligned}
$$

and $\overline{\mathrm{x}}_{\mathrm{ac}}=\overline{\mathrm{x}}_{r r}+\overline{\mathrm{x}}_{r}$, tracker equation

See Fig. $\mathrm{C}-1$.

The errors arise due to errors $\delta \overline{\mathrm{x}}_{r r^{\prime}} \delta \overline{\mathrm{x}}_{\mathrm{d}^{\prime}} \delta \overline{\mathrm{X}}_{r}$ and $\delta \overline{\mathrm{H}}$ in the specification of $\bar{X}_{r r}$ and in the measurements of $\bar{X}_{I M U}$ ' $\overline{\mathrm{X}}_{r}$ and $\overline{\mathrm{H}}$, respectively. The estimates (measurements) of $\overline{\mathrm{x}}_{\text {ter }}$ and $\overline{\mathrm{x}}_{\mathrm{ac}}$ derived from the actual position of the inertial system, tracker and profiler relate to the errors by the following equations:

C. 2 Terrain Measurement

$$
\overline{\mathrm{z}}_{\text {ter }}=\overline{\mathrm{x}}_{\text {ter }}+\delta \overline{\mathrm{x}}_{\text {ter }}
$$




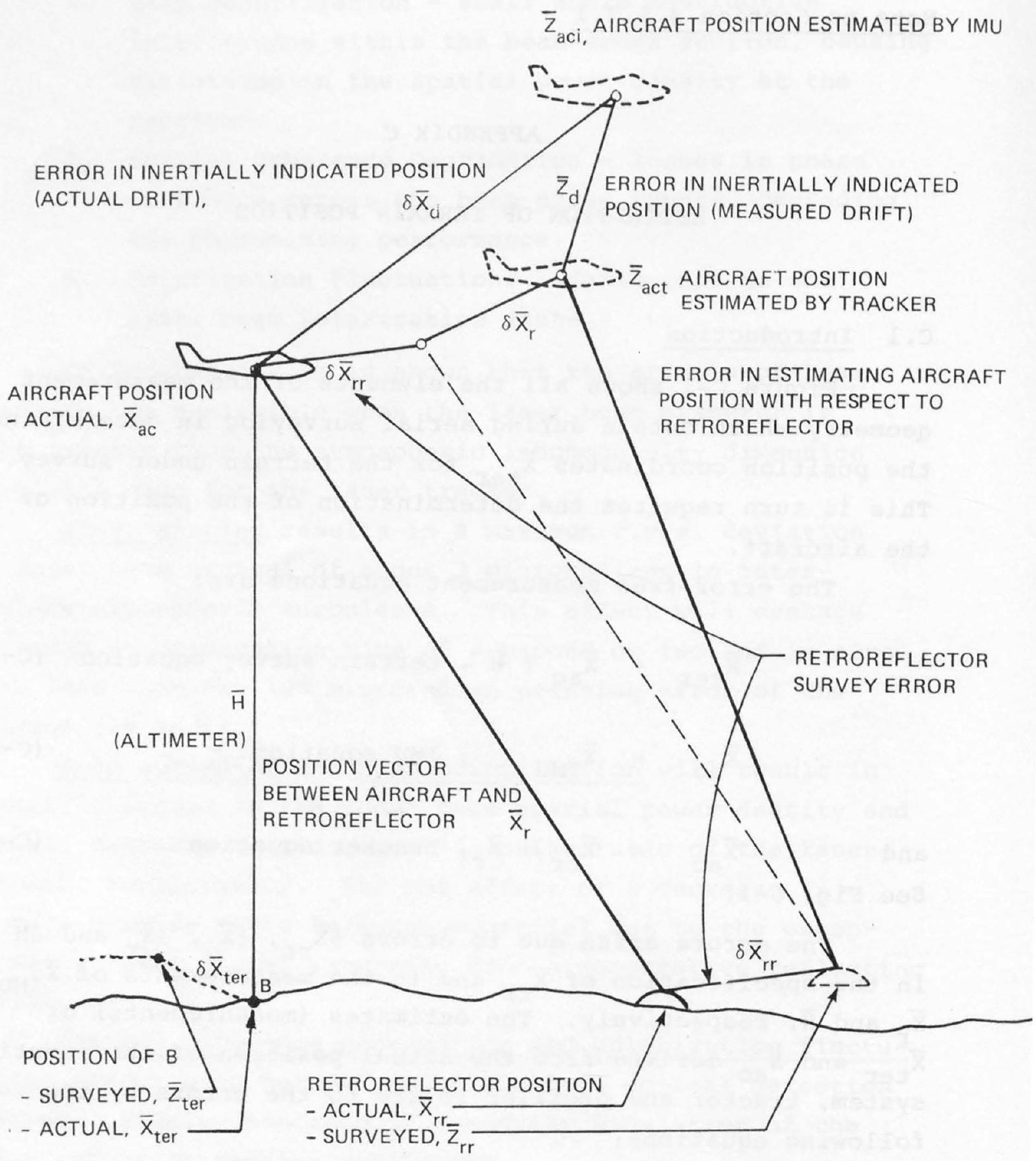

Fig. C-1. Measurement geometry during aerial surveying 
where

$$
\delta \overline{\mathrm{x}}_{\text {ter }}=\delta \overline{\mathrm{x}}_{\mathrm{ac}}+\delta \overline{\mathrm{H}}
$$

\section{3 Aircraft Measurement}

Inertial: $\quad \overline{\mathrm{Z}}_{\mathrm{ac}}=\delta \overline{\mathrm{x}}_{\mathrm{ac}}+\delta \overline{\mathrm{x}}_{\mathrm{d}}$

Tracker: $\quad \overline{\mathrm{z}}_{\mathrm{ac}}=\overline{\mathrm{x}}_{\mathrm{ac}}+\delta \overline{\mathrm{x}}_{r}+\delta \overline{\mathrm{x}}_{r r}$

\section{4 Inertial Error Measurement}

Subtracting Equations (C-7) from (C-6), we get

$$
\overline{\mathrm{z}}_{\mathrm{d}}=\delta \overline{\mathrm{x}}_{\mathrm{d}}-\delta \overline{\mathrm{x}}_{r}-\delta \overline{\mathrm{x}}_{r r}
$$

\section{C.5 Unsurveyed Retroreflector Measurement}

These equations are derived by combining the tracker and the inertially derived position measurements to yield $\overline{\mathrm{z}}_{\text {rr }}$ with

$$
\overline{\mathrm{z}}_{r r}=\overline{\mathrm{x}}_{r r}+\delta \overline{\mathrm{x}}_{\mathrm{d}}-\delta \overline{\mathrm{x}}_{r}
$$

It may be noted that the inertial position measurements are available at all times, whereas the tracker derived $\overline{\mathrm{z}}_{\mathrm{ac}}$ and $\overline{\mathrm{z}}_{\mathrm{d}}$ are available only during certain time intervals when the aircraft is within the coverage zone of a retroreflector.

The estimation problem thus involves estimating $\bar{x}_{\text {ter }}$, $\overline{\mathrm{x}}_{r^{\prime}}, \overline{\mathrm{x}}_{\mathrm{ac}}$ and $\delta \overline{\mathrm{x}}_{\mathrm{d}}$ from the derived measurements $\overline{\mathrm{z}}_{\text {ter }} \overline{\mathrm{z}}_{\mathrm{rr}}$ ' $\overline{\mathrm{z}}_{\mathrm{ac}}$ and $\overline{\mathrm{z}}_{\mathrm{d}}$.

We shall illustrate the mathematical considerations involved in the estimation of the above quantities by specifically considering the problem of estimating the inertial error vector $\delta \bar{x}_{\mathrm{d}}(t)$ from the measurements $\overline{\mathrm{z}}_{\mathrm{d}}$ obtained 
prior and subsequent to the particular time instant $t$. Although as stated above, the measurements $\bar{z}_{d}$ are available only during certain time intervals, the estimate of $\delta \bar{x}_{d}$ is desired at all times.

The measurement model is given by rewriting Equation $(\mathrm{C}-8):$

$$
\overline{\mathrm{z}}_{\mathrm{d}}=\delta \overline{\mathrm{x}}_{\mathrm{d}}+\bar{v}
$$

where

$$
\bar{v}=\delta \bar{x}_{r}+\delta \bar{x}_{r r}
$$

represents the noise in the measurements.

The dynamics of $\delta \bar{x}_{d}$ and the statistics of the noise need to be modeled before further consideration can be given to estimating $\delta \overline{\mathrm{x}}_{\mathrm{d}}$ (see Sec. 4.1).

The dynamics of $\delta \bar{x}_{d}$ and the nature of the noise $\bar{v}$ due to errors in the tracker measurements and the retroreflector sighting have been considered in detail in the previous sections and under section 4.1. The nature of inertial error growth $\delta \overline{\mathrm{x}}_{\mathrm{d}}$ has been considered for a general flightpath geometry in the presence of instrument and gravity model errors. For the sake of simplicity, we shall consider the problem of estimating the inertial error during a straightline flight path. For a straight-line flight path, the error growth is only a function of elapsed time since last position fix and is not a function of the flight-path geometry. The error growth of $\delta x$, the $x$ component of $\delta \bar{x}_{d}$ is given by rewriting Equation $(A-47)$ :

$$
\begin{aligned}
\delta x(t)= & k_{0}+k_{1} t+k_{2} t^{2}+k_{3} t^{3}+\nu_{x} . \\
& \text { for } t<\frac{t_{s}}{10}, t_{s}=84 \text { mins. }
\end{aligned}
$$


where $\mathrm{K}_{0}, \mathrm{~K}_{1}, \mathrm{~K}_{2}$ and $\mathrm{K}_{3}$ relate to the instrument biases, gravity model biases and the initialization errors as shown in $\mathrm{Eq}$. $(\mathrm{A}-47)$.

In Eq. $(C-11)$ the term $\nu_{x}$ represents the contribution of the components of the gravity and the instrument errors not represented in the terms $\mathrm{K}_{0}, \mathrm{~K}_{1}, \mathrm{~K}_{2}$ and $\mathrm{K}_{3}$. As long as the contribution of $\nu_{x}$ to $\delta x(t)$ is small, the estimation of the biases $\mathrm{K}_{0}, \mathrm{~K}_{1}, \mathrm{~K}_{2}, \mathrm{~K}_{3}$ would serve the purpose in the estimation of $\delta x(t)$.

In Eq. $(\mathrm{C}-10)$ the component of $\bar{v}$ due to tracker error is given by (see Sec, 4,1)

$$
\delta \overline{\mathrm{x}}_{r}=\frac{\overline{\mathrm{x}}_{r}}{\mathrm{r}} \times \delta r+\overline{\mathrm{x}}_{r} \times \bar{\psi}_{t}
$$

where

$$
\begin{aligned}
\delta r= & \text { the error in the measurement or range, } r, \\
& \text { between the aircraft and the retro- } \\
& \text { reflector }
\end{aligned}
$$

It may be remarked that the magnitude of $\bar{x}_{r} x \bar{\psi}_{t}$ is limited since $\bar{x}_{r}$ is the relative position of the aircraft with respect to the retroreflector, and thus is limited within a bounded zone of coverage. 
From Equation $(\mathrm{C}-3)$ we get

$$
\begin{aligned}
\overline{\mathrm{x}}_{r} & =\overline{\mathrm{x}}_{a c}-\overline{\mathrm{x}}_{r r} \\
\text { Let } \quad \overline{\mathrm{x}}_{\mathrm{ac}} & =\overline{\mathrm{x}}_{0}+\Delta \overline{\mathrm{x}}
\end{aligned}
$$

where $\quad \overline{\mathrm{x}}_{0}$ represents the position of the aircraft at the time of the last position update. Using $(C-14)$ in $(C-13)$ we get

$$
\bar{x}_{r} \simeq \bar{v}_{a c}{ }^{t}-\Delta \bar{x}_{r}
$$

where $\quad \Delta \overline{\mathrm{x}}_{r}=\overline{\mathrm{x}}_{r r}-\overline{\mathrm{x}}_{0}$

and $\Delta \overline{\mathrm{X}}=\overline{\mathrm{V}}_{\mathrm{ac}}{ }^{t}, t$ being the time elapsed since the last position update.

The other component of $\bar{v}$ due to the retroreflector siting error $\delta \overline{\mathrm{x}}_{\mathrm{rr}}$ is essentially a bias error.

Using $(A-47),(C-12)$ and $(C-15)$ in $(C-8)$, we get the following expression for $\mathrm{z}_{\mathrm{x} d^{\prime}}$ the $\mathrm{x}$ component of the measurement vector $\overline{\mathrm{z}}_{\mathrm{d}}$.

$$
z_{x d}=\left[1, t, t^{2}, t^{3}\right]\left[\begin{array}{c}
\dddot{\kappa}_{0} \\
\tilde{K}_{1} \\
\mathrm{~K}_{2} \\
\mathrm{~K}_{3}
\end{array}\right]+v
$$

where

$$
\begin{aligned}
& \tilde{\mathrm{K}}_{0}=\mathrm{K}_{0}+\delta \mathrm{x}_{\mathrm{rr}}-\Delta \mathrm{z}_{\mathrm{r}} \psi_{\mathrm{y} 0}-\Delta \mathrm{y}_{\mathrm{r}} \psi_{\mathrm{z} 0} \\
& \tilde{\mathrm{K}}_{1}=\mathrm{K}_{1}+\mathrm{v}_{\mathrm{Y}} \psi_{\mathrm{z} 0} \\
& \nu=\nu_{x}+\left(v_{y} t-\Delta y_{r}\right) \psi_{z e}-\Delta z_{r} \psi y_{e}
\end{aligned}
$$


In Equations $(C-17)$ and $(C-19),\left(\Delta y_{r^{\prime}}, \Delta z_{r}\right)$ and $\left(\psi_{y e} \psi_{z e}\right)$, respectively, represent the $y$ and $z$ axis components of $\Delta \bar{x}_{r}$ and $\bar{\psi}_{e}$. Equation $(\mathrm{C}-16)$ explicitly takes into account the effect of IMU frame misalignment in determining the orientation of angles of the line of sight vector $\bar{x}_{r}$.

\section{6 Estimation of $\mathrm{K}_{0}, \mathrm{~K}_{1}, \mathrm{~K}_{2}, \mathrm{~K}_{3}$}

The measurement $z_{i} \equiv z_{x d}\left(t=t_{i}\right)$ can be conveniently expressed in a compact notation as follows with

$$
\begin{aligned}
& z_{i}=H_{i} c+v_{i} \\
& C^{T}=\left[\tilde{k}_{0}, \tilde{k}_{1}, k_{2}, k_{3}\right] \\
& H_{i}=\left[1, t_{i}, t_{i}^{2}, t_{i}^{3}\right] .
\end{aligned}
$$

where

A batch of measurements made at times $t_{i}, i=1,2, \ldots n$, can be represented also as

$$
\left[\begin{array}{c}
\mathrm{z}_{1} \\
\mathrm{z}_{2} \\
\mathrm{z}_{\mathrm{n}}
\end{array}\right]_{\mathrm{nx1}}\left[\begin{array}{c}
-\mathrm{H}_{1}- \\
\vdots \\
\vdots \\
\hdashline \\
\hdashline
\end{array}\right]
$$

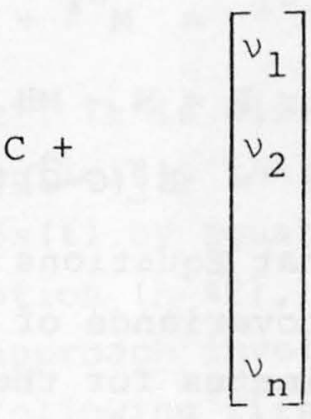

$\mathrm{nx} 1$

or as

$$
\mathrm{z}=\mathrm{HC}+\mathrm{v}
$$

with $z_{i}, H_{i}, v_{i}$, respectively, forming the $i^{\text {th }}$ row elements of $\mathrm{z}, \mathrm{H}$ and $v$. 
Now the problem of estimating the parameter vector $\mathrm{C}$ can be posed as follows:

\section{7 Problem}

Estimate $C$, given the following:

(1) Measurements:

$\mathrm{z}=\mathrm{HC}+\nu, \mathrm{n}$ measurements

(2) Noise statistics:

Mean: $\mathrm{E}[\nu]=0$

Covariances $E\left[\nu \nu^{\mathrm{T}}\right]=R($ nxn matrix $)$

(3) A priori estimates: $\hat{\mathrm{C}}_{0}=\mathrm{E}[\mathrm{C}],(4 \mathrm{xl}$ matrix)

$$
M=E\left[\left(C-\hat{C}_{0}\right)\left(C-\hat{C}_{0}\right) t^{t}\right. \text {, }
$$

(nxn matrix)

A weighted least-squares estimate of $C$ for the above problem is as follows:

Estimate: $\hat{\mathrm{C}}=\hat{\mathrm{C}}_{0}+\mathrm{K}\left[\mathrm{z}-\mathrm{H} \hat{\mathrm{C}}_{0}\right]$

$$
\text { Gain: } \mathrm{K}=\mathrm{PH}^{\mathrm{T}} \mathrm{R}^{-1}
$$

$(4 \mathrm{xn}$ matrix) $(\mathrm{C}-26)$

Estimate: $\mathrm{P}^{-1}=\mathrm{M}^{-1}+\mathrm{H}^{\mathrm{T}} \mathrm{r}^{-1} \mathrm{H}$ (4x4 matrix)

Error variance: or $P=M-M H\left(H_{M H}{ }^{T}+R\right)^{-1}$

where

$$
P=E\left[(C-\hat{C})(C-\hat{C})^{T}\right] \text {. }
$$

It may be noted that Equations $(\mathrm{C}-25)$ and $(\mathrm{C}-27)$ provide the estimate and the covariance of the estimate, which could form a priori estimates for the next batch of measurements. The size of the measurement vector can be as small as one measurement, in which case the estimate $\mathrm{C}$ is sequentially updated as each new measurement is obtained. In Equation (C-25) the measurement residuals, $\mathrm{z}-\mathrm{H} \hat{\mathrm{C}}_{0}$, are weighted by a gain matrix $\mathrm{K}$ which takes into account both the information and noise content of each measurement. For error measurements over a short time interval, only the lower order 
coefficients of the time polynomial of $(\mathrm{C}-16)$ could be effectively estimated, since the contribution of the higher order terms to the error growth could be minimal.

From the estimates of $\mathrm{K}_{0}, \mathrm{~K}_{1}, \mathrm{~K}_{2}$ and $\mathrm{K}_{3}$, a prediction of the inertial system error growth could be provided over those segments of the straight-line flight path over which ground truth data are not available. In order also to provide an estimate of the inertial system error growth for flight paths which are not straight, it becomes necessary to separate in the estimates of $\mathrm{K}_{0}, \mathrm{~K}_{1}, \mathrm{~K}_{2}$ and $\mathrm{K}_{3}$ the contribution of the gravity model, instrument error biases, and also the retroreflector siting error biases. Based on the considerations of previous sections on the effect of gravity model and instrument error biases on the inertial error growth, appropriate flight-path patterns can be devised to suppress or enhance the effect of the path dependent (space dependent) error growth terms, with respect to the non-path dependent (time dependent) error growth terms.

\section{8 Kalman Filter}

In the estimation of $\delta x(t)$, it is also possible to use Equation $(\mathrm{A}-42)$, rather than explicitly taking into account the dynamics of the IMU error $\delta \mathrm{x}(t)$ by Equation $(A-47)$ which represents the solution of Equation $(A-42)$,

We shall illustrate the approach involved in the estimation by considering the following first order system with the dynamics given by

$$
\begin{aligned}
& \dot{y}=b+u \\
& \dot{b}=0
\end{aligned}
$$

where $b$ represents an unknown bias and $u$, a gaussian white 
noise with

$E[u]=0$ and $E\left[u(t) u^{T}(\tau)\right]=Q \delta(t-\tau), \delta$ being $a$ Dirac delta function.

The measurements $z(t)$ are available continuously with

$$
z=y+v
$$

where $E[\nu]=0$ and $E\left[\nu(t) \nu^{T}(\tau)\right]=R \delta(t-\tau)$

The estimation problem can be stated as follows:

Estimate $y(t), b$,

Given

(i) $z(\tau), 0<\tau<t_{1}, t_{1}<t$

(ii) Initial estimates $\hat{\mathrm{y}}_{0}, \hat{\mathrm{b}}_{0}$ and

their covariance $M=E\left[\left(\begin{array}{l}y-\hat{y}_{0} \\ b-\hat{b}_{0}\end{array}\right)\left(y-\hat{y}_{0}\right)\left(b-\hat{b}_{0}\right)\right]$

The estimates of $y$ and $b$ are provided by the following Kalman filter equations

Estimator: $\quad \dot{\hat{C}}=F \hat{C}+K(z-H \hat{C})$

$$
\hat{\mathrm{C}}_{0}=\left[\begin{array}{l}
\hat{\mathrm{y}}_{0} \\
\hat{\mathrm{b}}_{0}
\end{array}\right]
$$

$$
\text { Gain: } K=\mathrm{PH}^{\mathrm{T}} \mathrm{R}^{-1}
$$

Error variance: $\dot{\mathrm{P}}=\mathrm{FP}+\mathrm{PF}^{\mathrm{T}}+\mathrm{Q}-\mathrm{PH}^{\mathrm{T}} \mathrm{R}^{-1} \mathrm{HP}$

$$
\mathrm{P}=\mathrm{M}
$$

where

$$
\begin{aligned}
& \mathrm{F}^{\mathrm{O}}=\left[\begin{array}{ll}
0 & 1 \\
0 & 0
\end{array}\right], \mathrm{H}=\left[\begin{array}{ll}
1,0
\end{array}\right] \text { and } \\
& \hat{\mathrm{C}}=\left[\begin{array}{l}
\hat{\mathrm{y}} \\
\hat{\mathrm{b}}
\end{array}\right]
\end{aligned}
$$


It may be remarked that Equation (C-31) provides the estimate of both $y$ and the bias $b$. The number of first order differential equations involved in the solution of $(\mathrm{C}-31)$ thus depends upon the order of the state of the system and the number and the order of the bias vectors that need to be estimated. The number of equations that need to be solved in $(C-33)$ is equal to $n x(n+1) / 2$, where $n$ is equal to the number of equations in $(c-31)$. The error variance equation is needed to calculate the Kalman gain matrix $\mathrm{K}$. The gain matrix $\mathrm{K}$ is a time-varying matrix which attains a steady state (constant) value at large $t$. However, a constant gain matrix in the estimator cannot be used because of the necessity to estimate bias vector $b$ before $k$ attains steady state.

It may be remarked that for $u=0, z=b t+v$, and the problem to estimate $b$ is easily handled under the leastsquare-estimate framework for parameters considered previously. The above estimation problem has been based in the filtering and prediction framework suitable for real time processing when the estimates are provided on the basis of past measurements only.

When both the past as well as future measurements (for $t_{1}>t$ ) are available, the equations (called smoothing equations) that provide the estimate of $y$ and $b$ are as follows:

Smoothing Equation: $\tilde{C}(t)=\hat{C}(t)-P(t) \lambda(t) \quad(C-34)$

where $P(t)$ and $\lambda(t)$ represent the solutions of $(c-33)$ and $(\mathrm{C}-35)$.

$$
\dot{\lambda}=-\left(\mathrm{F}-\mathrm{PH}^{\mathrm{T}} \mathrm{R}^{-1} \mathrm{H}\right) \lambda+\mathrm{H}^{\mathrm{T}} \mathrm{R}^{-1}(\mathrm{z}-\mathrm{H} \hat{\mathrm{x}}), \lambda\left(\mathrm{t}_{1}\right)=0
$$


The covariance of the error of the smoothed estimate $\widetilde{C}$ is given by $P(t)-P(t) \Lambda(t) P(t)$

where

$$
\begin{aligned}
\dot{\Lambda}=-\left(\mathrm{F}-\mathrm{PH}^{\mathrm{T}} \mathrm{R}^{-1} \mathrm{H}\right)^{\mathrm{T}} \Lambda & -\Lambda\left(\mathrm{F}-\mathrm{PH}^{\mathrm{T}} \mathrm{R}^{-1} \mathrm{H}\right) \\
& +\mathrm{H}^{\mathrm{T}} \mathrm{R}^{-1} \mathrm{H}, \Lambda\left(\mathrm{t}_{\mathrm{f}}\right)=0 \quad(\mathrm{C}-36)
\end{aligned}
$$


GEOMETRICAL ASPECTS OF UPDATING THE IMU WITH THE LASER TRACKER

\section{D.1 The IMU Update Problem}

The origin and nature of the performance errors unique to the gyro and accelerometer components of the IMU have been individually described, analyzed, and discussed in other parts of this report. These errors form the hard core of knowledge for deciding what information must be fed to the IMU in flight to ensure that it performs continuously within the overall stipulated accuracy standards. The decision to use state-of-the-art gyros and accelerometers in the IMU helps set the precision and frequency with which that information must be supplied.

Because it is fundamental not only to the design features of the instrument system itself, but also to the development of workable flight-operating techniques, the procedure for updating the IMU is described in this appendix which includes the necessary supporting mathematical analysis.

The nature of the updating problem emerges from a realization that despite use of state-of-the-art gyros and accelerometers the set of 3-coordinate axes (the reference frame) being carried by the IMU very slowly rotates away from its true alignment, giving rise to slow growths in error in the orientation as well as 3-coordinate position that the IMU indicates. How to observe this departure from 
the truth, with measurements permitting the IMU to be correctly reset in flight, is the challenge of the updating problem.

\section{2 Geometrical Development}

In order to determine the updating procedure for the inertial system, we may start by defining the following coordinate frames, all of which are right-handed, orthogonal and rectangular (see Fig. 4,1-3):

1) The "e" frame (for "earth") fixed to the rotating earth, with its origin at the center of the earth.

$$
\begin{aligned}
& z_{e}=\text { along the north polar axis } \\
& x_{e}=\text { intercepts the prime meridian }
\end{aligned}
$$

2) The "n" frame (for "near") fixed to the rotating earth. The origin of the " $n$ " frame is at the location of the retroreflector being tracked.

$$
\begin{aligned}
z_{n}= & \text { downward along the normal to the refer- } \\
& \text { ence ellipsoid that passes through the } \\
& \text { retroreflector } \\
x_{n}= & \text { north } \\
y_{n}= & \text { east }
\end{aligned}
$$

3) The "g" frame (for "geographic") is a verticaltracking reference frame with its origin fixed in the IMU.

$$
\begin{aligned}
z_{g}= & \text { downward along the normal to the refer- } \\
& \text { ence ellipsoid that passes through the } \\
& \text { IMU }
\end{aligned}
$$




$$
\begin{aligned}
& x_{g}=\text { north } \\
& y_{g}=\text { east }
\end{aligned}
$$

4) The "p" frame (for "platform") is chosen by definition to be fixed to the inner or stable member of the IMU. It is defined as being coincident with the "g" frame for zero IMU errors.

The inertial navigator provides a time history of position coordinates of the aircraft. The sequence of coordinate positions can be thought of as tracing out the flight path in space, in reference to a known coordinate frame (say the "e" frame), along which the system thinks the aircraft is travelling. One can represent the indicated aircraft position, at any instant of time, by a vector from the center of the earth to the indicated position coordinates in the "e" reference frame. For simplicity, choose the known reference point in the "e" frame to be the retroreflector location at the origin of the "n" frame. Then

$$
\begin{aligned}
\overleftarrow{\mathrm{R}}= & \text { vector from retroreflector to aircraft as } \\
& \text { computed from }
\end{aligned}
$$

a) surveyed coordinates of retroreflector

b) output coordinates of navigator (IMU)

It is assumed that there is no error, called $\delta \bar{x}_{r r}$ in Fig. 4.1-2, in the surveyed position of the retroreflector and no error called $\delta \bar{x}_{r}$ in Fig. 4.1-2, in estimating the position of the aircraft with respect to the retroreflector (see Fig, $D-1)$. Let the coordinates of $\bar{R}$ be denoted by

$$
r_{x}, r_{y}, r_{z}
$$

The actual position of the aircraft is $\bar{R}_{A}$ (called $\bar{x}_{\text {ac }}$ in Fig. 4.1-2) and its n-frame coordinates are 


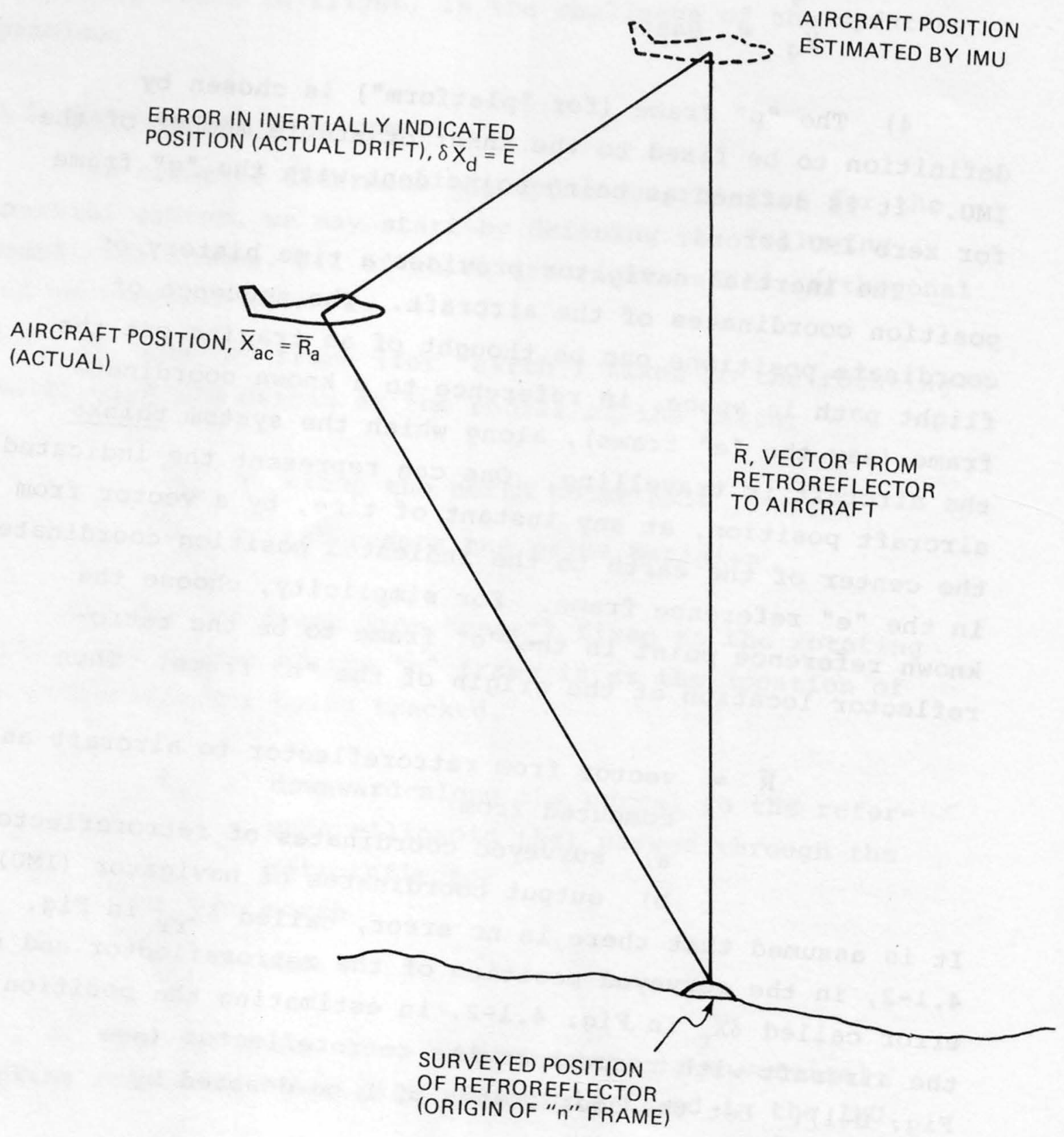

Fig. D-1. Geometry of aircraft and retroreflector used in laser tracker 


$$
r_{A x_{n}}, r_{A y_{n}}, r_{A z_{n}}
$$

The position error $\overline{\mathrm{E}}$ (called $\delta \overline{\mathrm{x}}_{\mathrm{d}}$ in Fig. 4.1-2) is defined to be the set of differences between these sets of coordinates,

$$
\begin{aligned}
& e_{x}=r_{x}-r_{A x_{n}} \\
& e_{y}=r_{y}-r_{A y_{n}} \\
& e_{z}=r_{z}-r_{A z_{n}}
\end{aligned}
$$

from which

$$
\begin{aligned}
& e_{x}+r_{A x_{n}}=r_{x} \\
& e_{y}+r_{A y_{n}}=r_{y} \\
& e_{z}+r_{A z_{n}}=r_{z}
\end{aligned}
$$

Since $\bar{R}$ is known, if $\bar{R}_{A}$ were known we could compute $\bar{E}$, the position error of the aircraft at any instant of time, and from the time history of $\overline{\mathrm{E}}$ estimate the various parameters of the system error model (accelerometer bias, gravity gradient effect, etc.).

To get $\bar{R}_{A}$ we need independent information. We use the IMU mounted tracker, since this will give us a vector measurement from the true location of the aircraft to the true location of the retro. The scalar range measurement. of the tracker is resolved into vector components, using the 
tracker gimbal angle readouts. This, however, introduces a problem. The vector components obtained are resolved along the axes of the "p" frame (the only thing possible), whereas the "n" frame coordinates of $\bar{R}_{A}$ are needed. The latter are obtained from the former by

$$
\begin{aligned}
& r_{A x_{n}}=C_{11}{ }_{A x_{p}}+C_{12}{ }^{r_{A y}}+C_{13}{ }^{r_{A z}} \\
& r_{A y_{n}}=C_{21}{ }^{r_{A x}}+C_{22}{ }^{r_{A y}}+C_{23}{ }^{r} A_{p} \\
& { }^{r_{A z_{n}}}=C_{31}{ }^{r_{A x}}+C_{32}{ }^{r_{A y}}+C_{33}{ }^{r_{A z}}
\end{aligned}
$$

where the $C_{i j}$ are the direction cosines between the two reference frames.

Then

$$
\begin{aligned}
& e_{x}+c_{11} r_{A x}+c_{12}{ }^{r} A_{p}+c_{13}{ }^{r} A_{p}=r_{x} \\
& e_{y}+c_{21} r_{A x}+c_{22}{ }^{r} A_{p}+c_{23}{ }^{r} A_{p}=r_{y}(D-3) \\
& e_{z}+c_{31} r_{A x}+c_{32}{ }^{r} A_{p}+C_{33}{ }^{r} A z_{p}=r_{z}
\end{aligned}
$$

Subtracting the $\mathrm{p}$-components of $\overline{\mathrm{R}}_{\mathrm{A}}$ from both sides,

$$
\begin{aligned}
& e_{x}+\left(C_{11}-1\right) r_{A x_{p}}+c_{12} r_{A y_{p}}+C_{13} r_{A z_{p}}=r_{x}-r_{A x_{p}} \\
& e_{y}+C_{21} r_{A x}+\left(C_{22}-1\right) r_{A y_{p}}+C_{23} r_{A z_{p}}=r_{y}-r_{A y_{p}} \quad(D-4) \\
& e_{z}+C_{31} r_{A x}+C_{32}{ }^{r} A_{p}+\left(C_{33}-1\right) r_{A z_{p}}=r_{z}-r_{A z}
\end{aligned}
$$


It can be shown by a first order perturbation technique and the small angle approximation that

$$
\begin{aligned}
& c_{11}-1=C_{22}-1=c_{33}-1=0 \\
& c_{32}=-C_{23}=\psi_{x} \\
& c_{13}=-C_{31}=\psi_{y} \\
& c_{21}=-C_{12}=\psi_{z}
\end{aligned}
$$

where $\psi_{x^{\prime}} \psi_{y^{\prime}} \psi_{z}$ are the components of the small rotation vector between the two reference frames. Thus (D-4) becomes

$$
\begin{aligned}
& e_{x}-r_{A y_{p}} \psi_{z}+r_{A z_{p}} \psi_{y}=d_{x} \\
& e_{y}+r_{A x_{p}} \psi_{z}-r_{A z_{p}} \psi_{x}=d_{y} \\
& e_{z}-r_{A x_{p}} \psi_{y}+r_{A y_{p}} \psi_{x}=d_{z}
\end{aligned}
$$

where the d's are the right sides of the equations. Letting

$$
\bar{E}=\left[\begin{array}{l}
e_{x} \\
e_{y} \\
e_{z}
\end{array}\right], \quad \bar{\psi}=\left[\begin{array}{l}
\psi_{x} \\
\psi_{y} \\
\psi_{z}
\end{array}\right], \quad \bar{R}=\left[\begin{array}{l}
r_{A x_{p}} \\
r_{A y} \\
r_{A z_{p}}
\end{array}\right], \quad \bar{D}=\left[\begin{array}{l}
d_{x} \\
d_{y} \\
d_{z}
\end{array}\right]
$$

equations (D-6) may be given in vectorial form as

$$
\overline{\mathrm{E}}+\bar{\Psi} \times \overline{\mathrm{R}}=\overline{\mathrm{D}}
$$


Here

$\overline{\mathrm{E}}$ is the inertial position error vector

$\overline{\mathrm{R}}$ is the measured vector range to the retroreflector

$\overline{\mathrm{D}}$ is the difference between the calculated and the measured vector range to the retro,

Thus, a single sighting on a single retro would allow us to solve for either $\overline{\mathrm{E}}$ or $\bar{\Psi}$ (but not the line of sight component of $\bar{\Psi}$ ). It is not possible, however, to solve for both of these vectors from a sighting on a single retro.

Both $\overline{\mathrm{E}}$ and $\bar{\Psi}$ can be modelled by a time series with constant coefficients and a statistical estimate of the coefficients can be made from a time series of measurements on the same retroreflector. The estimated coefficients will, however, be an unresolvable combination of the coefficients of the $\overline{\mathrm{E}}$ model and the $\bar{\Psi}$ model since they both exhibit similar time dependence.

If two retroreflectors are sighted simultaneously from the aircraft, then there are 6 equations and 6 unknowns. The two-retroreflector situation is depicted in the following figure:

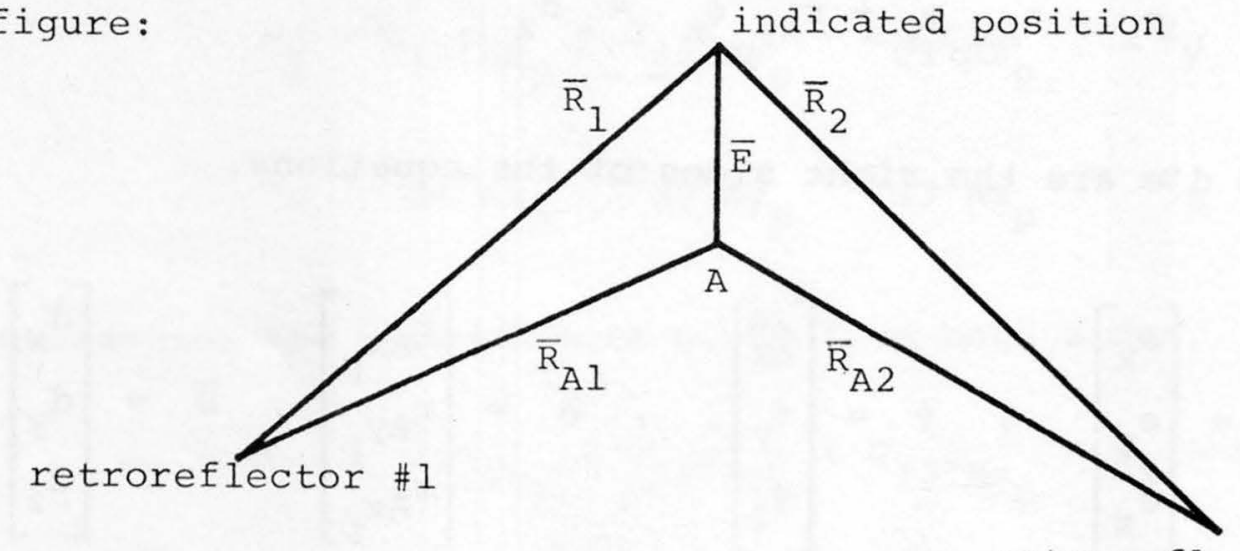

from which

retroreflector \#2

$$
\begin{aligned}
& \overline{\mathrm{E}}+\bar{\Psi} \times \overline{\mathrm{R}}_{1}=\overline{\mathrm{D}}_{1} \\
& \overline{\mathrm{E}}+\bar{\Psi} \times \overline{\mathrm{R}}_{2}=\overline{\mathrm{D}}_{2}
\end{aligned}
$$


which comprises the 6 equations in the 6 unknowns $\overline{\mathrm{E}}$ and $\bar{\Psi}$. $\overline{\mathrm{E}}$ is the error in the inertially indicated position. It is expressed in the " $n$ " frame, so it is the "complete" error, including the effects of the inertial frame misalignment.

$\bar{\Psi}$ actually has two components:

1) the small difference between the orientation of an "n" frame with its origin at the retro, and a "g" frame with its origin at the aircraft.

2) the misalignment of the "p" frame with respect to the "g" frame at the aircraft.

$\bar{R}_{1}$ and $\bar{R}_{2}$ are the range vector measurements by the tracker, referenced to the on-board "p" frame. $\bar{R}_{1}$ and $\bar{R}_{2}$ are expressed in the "p" frame.

$\overline{\mathrm{D}}_{1}$ and $\overline{\mathrm{D}}_{2}$ are the vector differences between the computed range vectors (from the inertially indicated position) and $\bar{R}_{1}$ and $\bar{R}_{2}$, respectively. The computed range vectors are expressed in the " $n$ " frame.

It can be shown that the six equations are not independent due to the fact that the vector $\bar{R}_{p 1}-\bar{R}_{p 2}$ is known and there exists an infinite family of solutions relating $\bar{\Psi}$ and $\overline{\mathrm{E}}$ that will satisfy the conditions defined or described by Eqs. $(D-8)$ and $(D-9)$. Additional information is needed either in the form of a third measurement or another condition in $\bar{\Psi}$ or $\overline{\mathrm{E}}$.

A third retroreflector would provide the vector $\bar{R}_{3}$ and the equation:

$$
\overline{\mathrm{E}}+\bar{\Psi} \times \overline{\mathrm{R}}_{3}=\overline{\mathrm{D}}_{3}
$$

Equations (D-8), (D-9) and $(D-10)$ would then yield unique solutions for $\bar{\Psi}$ and $\bar{E}$ (provided the retroreflectors are not collinear). 
An example of the other alternative, a totally different type of condition, might be drawn from the knowledge, say, that misalignment between the " $p$ " frame and "g" frame was entirely about the vertical (azimuth only). A unique solution of $(D-8)$ and $(D-9)$ for $\bar{\Psi}$ and $\bar{E}$ would then be possible.

One of the really strong advantages of the inertial system becomes apparent when the above-mentioned requirement of simultaneity is examined more closely. In practice, $\bar{E}$ and $\bar{\Psi}$ are not solved for in the simple deterministic fashion indicated. In fact, both of these vectors are modelled by a time series:

$$
\begin{aligned}
& \bar{E}=\bar{E}_{0}+\bar{E}_{1} t+\bar{E}_{2} t^{2}+\cdot . \cdot \\
& \bar{\Psi}=\bar{\Psi}_{0}+\bar{\Psi}_{1} t+\bar{\Psi}_{2} t^{2}+\cdot \cdot \cdot
\end{aligned}
$$

The object of the solution is now the coefficients in the above models. The reason for doing this is that the coefficients are invariant over times on the order of the duration of the data gathering mission, Therefore, a time sequence of measurements which is used to solve for these coefficients can be considered simultaneous as long as the model is valid over the specified time interval. Thus a discrete, time separated, series of measurements can be used and still satisfy the requirement of simultaneity. Therefore, only a single tracker is required.

It is important to note that since the inertial high frequency errors are negligible, the model equations (D-11) and (D-12) may be quite truncated, It is thought that four coefficients in (D-11) and two coefficients in (D-12) would be the maximum required at any time.

Since the coefficients are themselves vectors, a set 
of $\mathrm{k}$ coefficients would require $3 \mathrm{k}$ equations or $\mathrm{k}$ observations. Thus, 6 coefficients would require a minimum of 6 independent observations. Since the retroreflectors will be tracked over fairly wide angles, many more than 6 independent observations will be obtained, and the redundant information will be statistically processed to improve the accuracy of the estimates, in the presence of tracker and atmospheric noise.

It is important to note that the additional "degrees of freedom" (or unknowns) introduced by the modelling are with respect to time only - and not with respect to space as compared to the simple deterministic problem expressed, for example, by $(D-8),(D-9)$ and $(D-10)$. Therefore, the geometric requirement described above still holds, when $(D-11)$ and $(D-12)$ are employed. The time dependent information may come from a sequence of measurements on the same retros.

\section{D.3 Magnitudes of Error Quantities}

An examination of the expected magnitudes of $\overline{\mathrm{E}}$ and $\bar{\psi}$ is fruitful. Assuming the overall system is operated in accord with the specified surveying precision standards, $\overline{\mathrm{E}}$ will be on the order of 10 feet in the horizontal channels and $\frac{1}{2}$ foot in the vertical. The horizontal position errors of an inertial system may be assumed to be approximately related to the horizontal platform misalignment angles by

where

$$
\psi_{h}=e_{h} / r_{0}
$$

$\psi_{h}=$ horizontal platform misalignment

$e_{h}=$ horizontal position error

$r_{0}=$ radius of the earth 
Using

$$
\begin{aligned}
& e_{h}=10 \mathrm{ft} . \text { we get } \\
& \psi_{h} \simeq 0.1 \mathrm{sec} .
\end{aligned}
$$

so that horizontal position errors of $10 \mathrm{ft}$, if they are caused by gyro drift, would be associated with platform misalignment angles on the order of 0.1 sece. It should be pointed out that the estimation procedure is firmly based on geometry alone, however, and makes use of no such presumptuous relation as $(\mathrm{D}-13)$.

The vertical or azimuth misalignment, $\psi_{V^{\prime}}$ gives rise to a horizontal position error where the relation is

$$
\psi_{V}=\frac{e_{h}}{\Delta r}
$$

where

$$
\begin{aligned}
& \psi_{\mathrm{V}}=\text { "azimuth" misalignment error } \\
& \Delta \mathrm{r}=\text { distance from a "position reset" }
\end{aligned}
$$

Thus for $\Delta r=20$ miles and $e_{h}=10$ feet. $\psi_{V}$ is on the order of 19 sec.

It could further be observed that the vertical position error is relatively independent of small platform misalignments, being chiefly a function of vertical accelerometer bias error or vertical gravity gradients. This, in fact, is probably the spacing requirement of retroreflectors, where the significant relation is given by

$$
e_{v}=\frac{1}{2} a t^{2}
$$

where

$$
e_{v}=\text { inertial error in the vertical channel }
$$




$$
\begin{aligned}
\mathrm{a}= & \text { total equivalent vertical channel bias } \\
& \text { error } \\
t= & \text { equivalent time between updates }
\end{aligned}
$$

Using $e_{v}=1.0$ foot and " $\mathrm{a} "=1.0 \mu \mathrm{g}$, we obtain $t$ on the order of $250 \mathrm{sec}$. This would meet the error specification, based on the assumption that the smoothed error would in fact be a maximum of $\frac{1}{2}$ foot, halfway in between the two retroreflectors. 

Note to Section 4.1 .6

\section{APPENDIX E}

COMPUTER SIMULATION OF ERROR GROWTH IN AN IMU

\section{E.1 Error Equations}

The equation solved by the inertial navigation system to determine aircraft position is little more than the standard kinematic equation for the acceleration of a rigid body. This equation, shown implemented in Fig. E-l, is:

$$
\ddot{\bar{x}}_{C}=\bar{A}-\dot{\bar{\omega}} \times \bar{x}_{i}-\bar{\omega} \times\left(\bar{\omega} \times \bar{x}_{i}\right)+\bar{g}\left(\bar{x}_{i}\right)-2 \bar{\omega} \times \bar{x}_{C}
$$

where

$$
\begin{aligned}
\dot{\overline{\mathrm{X}}}_{\mathrm{C}}= & \text { derivative of the magnitude of the components } \\
& \text { of } \overline{\mathrm{x}}_{i} \\
\overline{\mathrm{A}}= & \text { accelerometer output } \\
\bar{\omega}= & \text { rotation rate of navigation frame with respect } \\
& \text { to inertial frame } \\
\overline{\mathrm{x}}_{i}= & \text { position of IMU (platform) in inertial frame } \\
\bar{g}\left(\overline{\mathrm{x}}_{i}\right)= & \text { fixed with respect to stars }
\end{aligned}
$$

Performing a first order perturbation on Eq. (E-1), as explained in Refs. 2 and 9, and considering the platform angular errors, Eqs. $(E-2)$ and $(E-3)$ are derived that are shown in Fig. E-2.

Equation (E-2) gives the angular errors in the IMU as a function of time. Equation (E-3) gives positional errors 


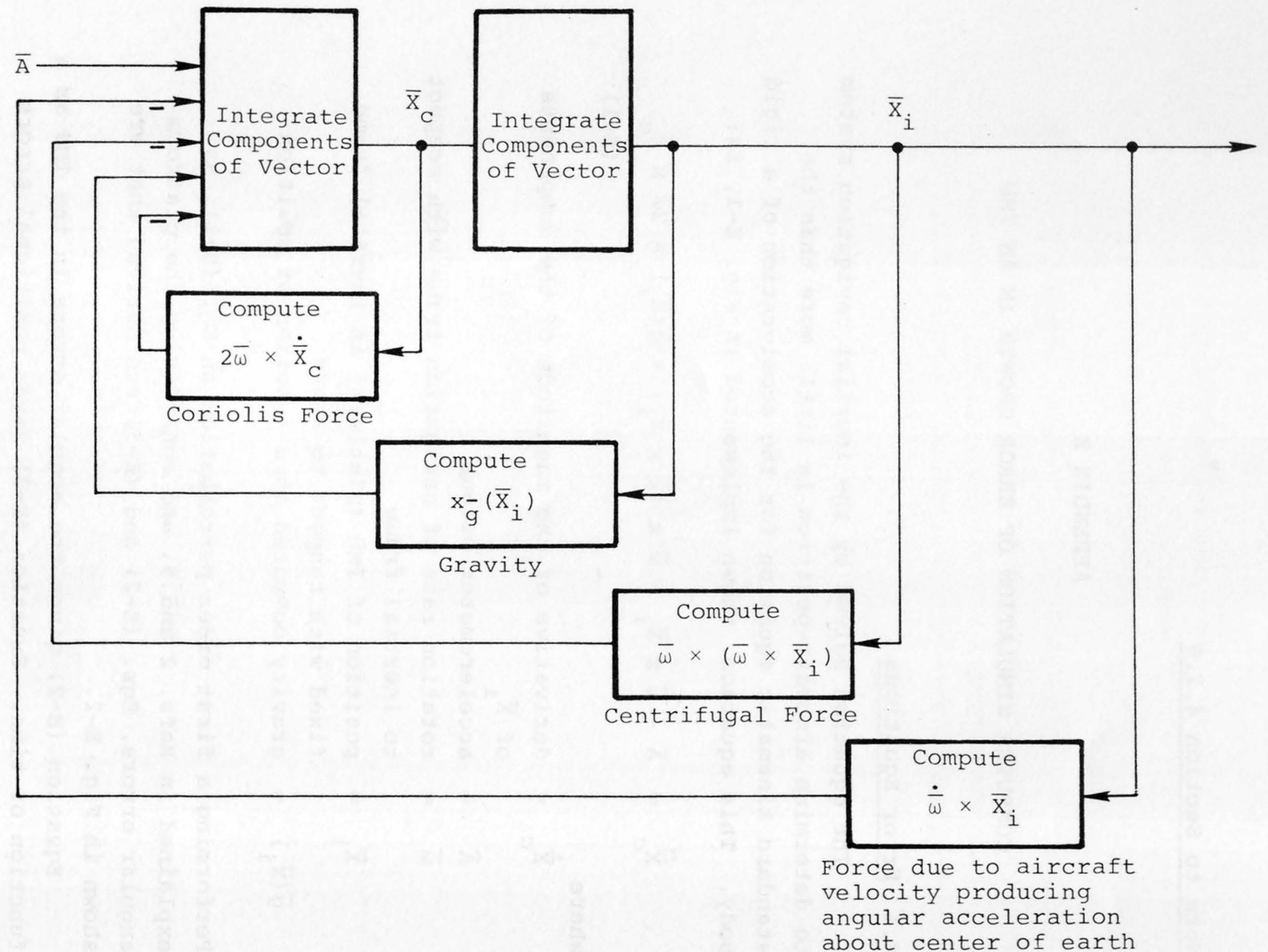

Fig. E-1. Inertial measurement system 
also as a function of time. The errors are interrelated as explained in Sect. 4.1.2.3 and Appendix A. The equations in that section and appendix are with reference to the geographic reference frame. With the addition of the components of the earth rotation rate, $\bar{\omega}$, these equations become referenced to the inertial frame.

Equation (E-3) follows from Eq. (E-1). Initially $\psi$ is 0 , but it builds up with time because of accelerations along the axis of the IMU. The term $\bar{A}$ in Eq. $(E-1)$ expands to $\bar{\psi} \times \overline{\mathrm{A}}+\overline{\mathrm{B}}+\stackrel{\mathrm{K}}{\mathrm{a}}_{\mathrm{A}} \mathrm{A}$. Equation $(\mathrm{E}-3)$ is $\mathrm{Eq} .(4 \cdot 1-6)$ with the addition of components of the earth rotation rate, $\bar{\omega}$, to relate the platform (navigation) frame to the inertial frame. The inputs to the differential equations are the gyro drift rates, mass unbalance errors, and torquing errors; and the accelerometer bias and scale factor errors. These biases are assumed constant over the time period of the flight. The values of the bias errors used in the simulation are:

$\mathrm{z}$ accelerometer bias $=1 \mu \mathrm{g}$

$\mathrm{x}, \mathrm{y}$ accelerometer bias $=10 \mu \mathrm{g}$

Accelerometer scale factor error $=10^{-6}$

Gyro drift rate uncertainty $=0.1$ meru

Gyro scale factor error $=2 \times 10^{-6}$

Gyro mass unbalances $=0.5 \mathrm{meru} / \mathrm{g}$

[one meru $\simeq .015^{\circ} / \mathrm{hr}$ ]

Since the analysis is linear, results for other higher or lower performance sets are approximated by dividing or multiplying by the assumed factor.

The inputs, during simulation, to the error equations that are functionf of time are the aircraft acceleration along the take-off and flight path and the gravity anomalies 


\begin{tabular}{|c|c|}
\hline 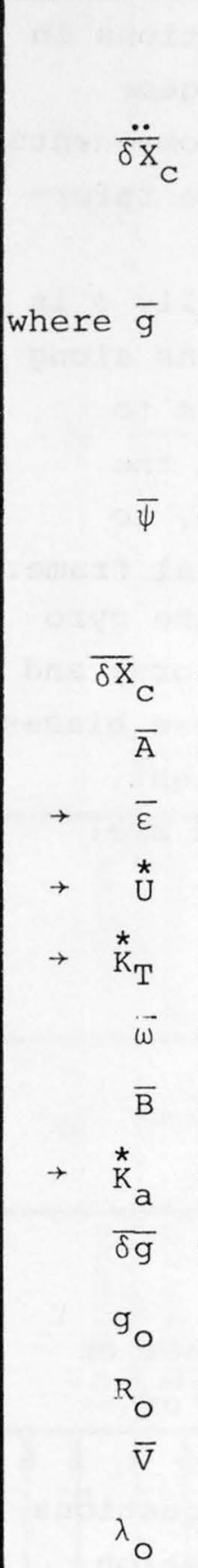 & 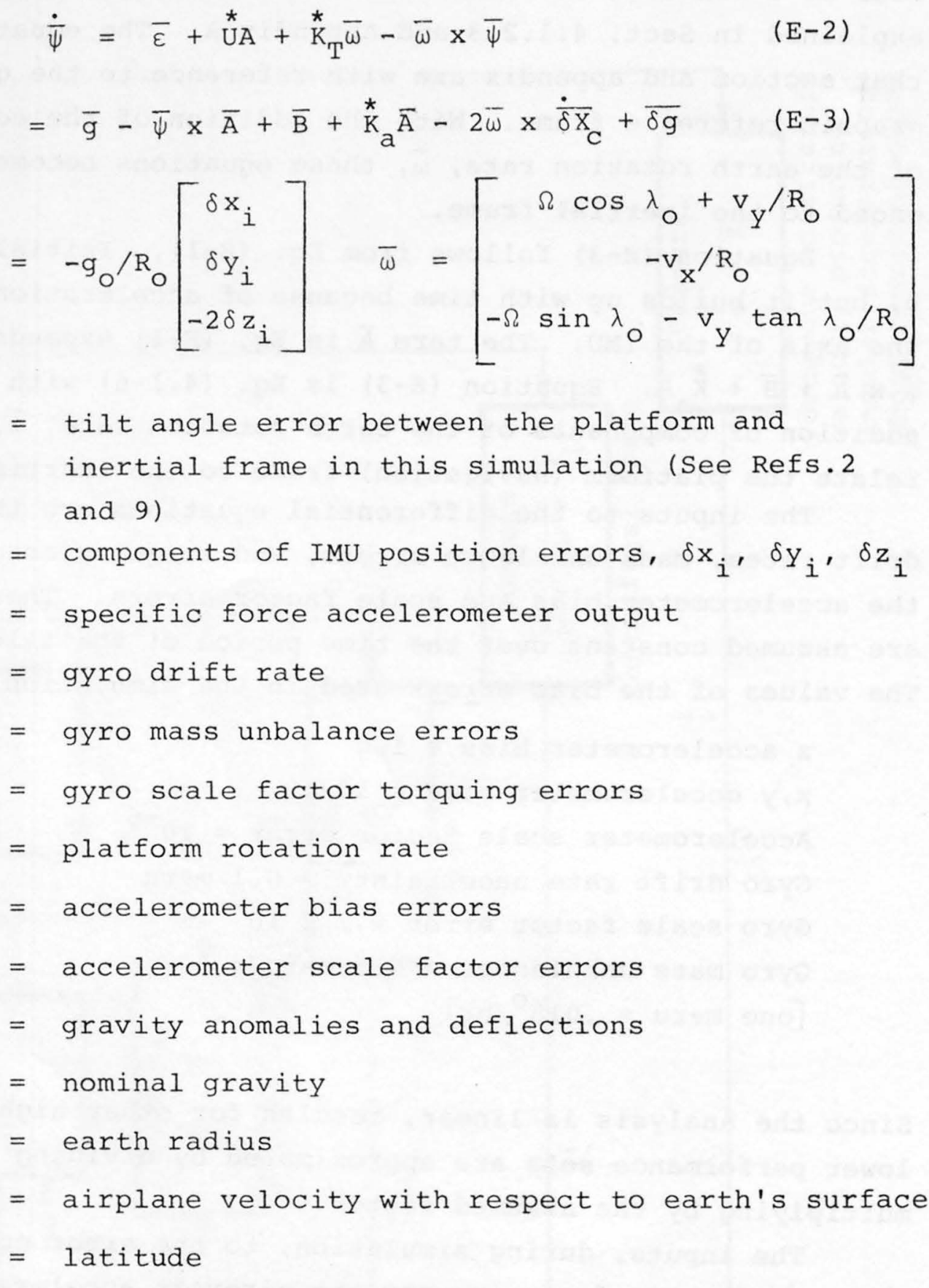 \\
\hline
\end{tabular}

Fig. E-2. Equations for error growth in an IMU. (The arrows at the left mark error sources referred to in Sect. E-5. The variable A is defined at the end of Section E.4.) 
and deflections. In reality the gravity anomalies and deflections are functions of the position of the aircraft. These data can be transferred to the time domain from knowledge of the aircraft flight path.

The flight path diagrams are Figures $4.1-8$ and 4.1-9, described in section 4.1.5.

\section{E. 2 Wind Gust Errors}

Simulation of the wind gust produced error in the aircraft position is based on the treatment of Etkin, Dynamics of Flight. (10) By determining the acceleration response function for a small aircraft and the input power spectrum of the wind gusts, it is possible to multiply these two curves together and compute the power spectrum of the wind gust acceleration. The area under this curve is proportional to the mean square gust acceleration, which for a "gusty" day is about $0.2 \mathrm{~g}(\mathrm{rms})$ and $0 . \mathrm{gg}(\mathrm{rms})$ in the $\mathrm{z}$ and $\mathrm{x}$ or $\mathrm{y}$ directions, respectively. It is also possible to determine the correlation time of the wind gusts; i.e., for what length of time does a gust result in an acceleration input to the accelerometers. Next, a computer generated random number routine is produced with a power spectrum, rms gust acceleration, and correlation time approximating as closely as possible the true wind noise values. The resultant random numbers are the accelerations added to the accelerations in the error propagation equation. The net effect is an increase in position error of about $1.0 \%$. This is as expected because of the good high frequency response of the inertial measurement unit.

\section{E. 3 Gravity Anomaly Simulation}

Gravity anomalies and deflections are incorporated into the error growth equations by adding the gravity anomaly 
as a function of time to the $z$ channel and the deflections multiplied by $g_{0}$ to the $x$ and $y$ channels. It became immediately obvious that for extreme anomaly locations such as the Des Moines, Iowa, area the resulting error growth exceeds the specified error margins. Thus, the simplest possible model of the anomalies was chosen. It is assumed that the gravity anomalies are known at three points (the three retroreflectors) and straight lines are drawn through these points to approximate the true anomalies. The difference between the true anomaly and the straight-line approximation is the acceleration input to the error equation as shown in Figure E-3. In other words, it is this difference in the computed and actual gravity that results in position errors in the inertial navigation system.

A plot of the gravity anomaly and deflection with the straight-line approximations is shown in Figs. $A-3$ and $A-4$ in Appendix A. Figure A-3, showing the anomalies in the Denver area, respresents a typical anomaly, whereas Fig. A-4 of the Des Moines area is a severe case.

\section{E. 4 Error Growth}

For a two- by twenty-mile rectangular strip, the larger the number of surveyed and unsurveyed retroreflectors, the smaller the time interval between retroreflectors. Tabulated in Section 4.1.5, Tables 4.1-2 through 4.1-5 are the allowable errors for the initial longitudinal pass and the allowable errors in the profiling mode. For the vertical channel (Tables 4.1-2 and 4.1-4) the errors grow according to Eq. $(\mathrm{E}-4)$. Using the linear plus quadratic plus cubic in time error terms of Eq. $(E-4)$, it is possible to calculate the allowable error source magnitudes as a function of time between retroreflectors. 


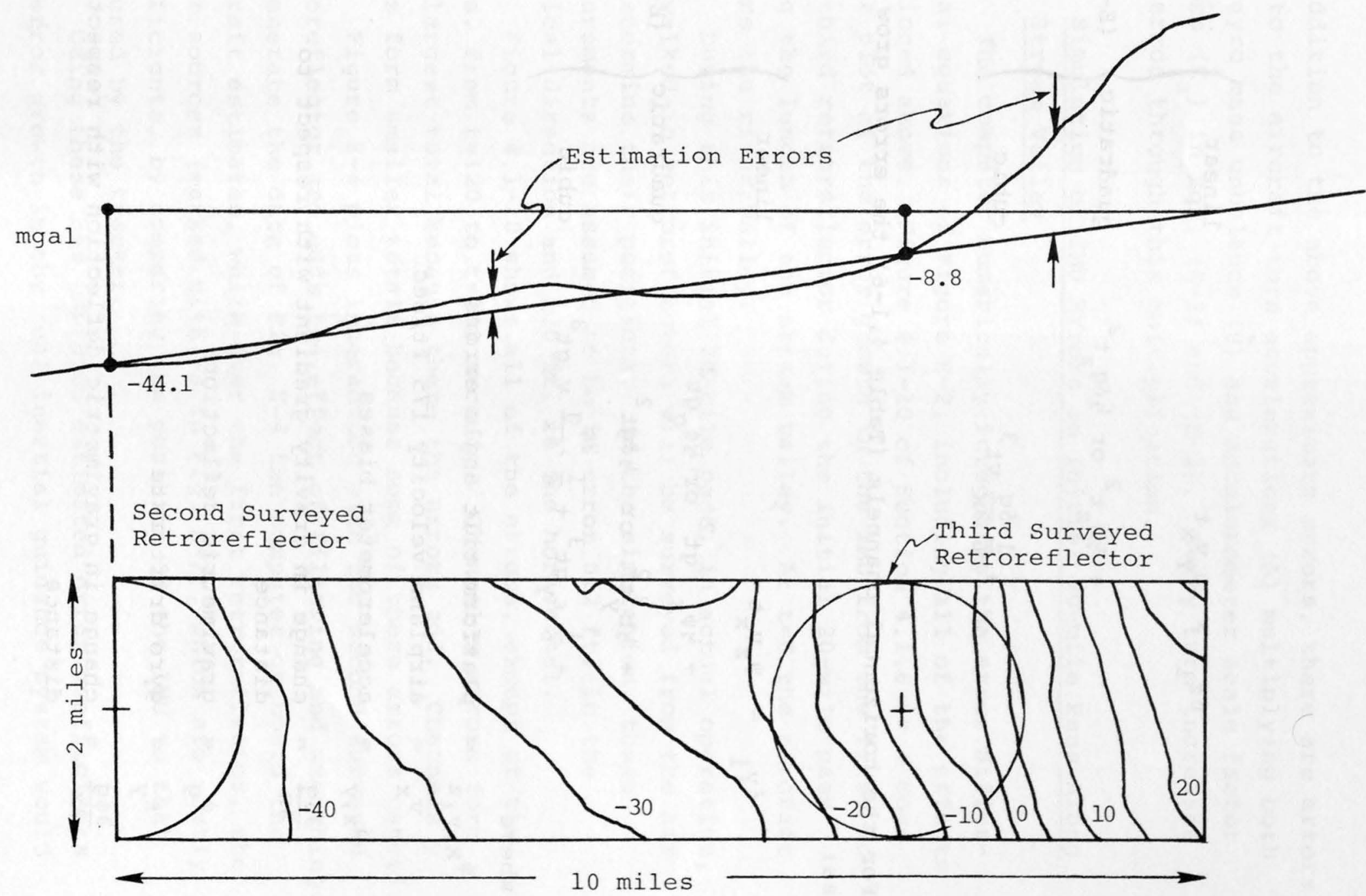

Fig. E-3. Gravity anomaly estimation technique 


$$
\begin{aligned}
\delta z_{i}= & \psi_{y} v_{x} t \\
& +\frac{1}{2} b_{z} t^{2} \text { or } \frac{1}{2} \delta g_{z} t^{2} \\
& +\frac{1}{6} \frac{\delta g}{\partial x} v t^{3}
\end{aligned}
$$

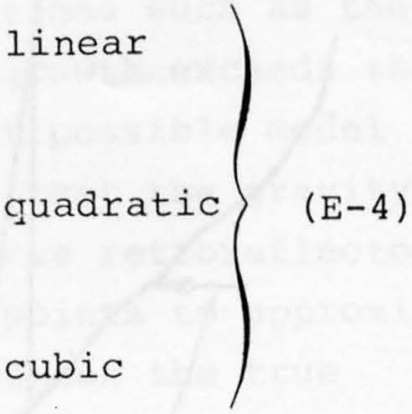

For the horizontal channels (Table 4,1-6) the errors grow as :

$$
\begin{aligned}
\delta y_{i}= & \psi_{z} v_{x} t \\
& +\frac{1}{2} \psi_{x} g t^{2} \text { or } \frac{1}{2} \phi_{g} g t^{2} \\
& +\frac{1}{2} b_{y} t^{2} \text { or } \frac{1}{2} \delta g t^{2} \\
& +\frac{1}{6} \varepsilon_{y} g t^{3}+\frac{1}{6} \frac{\partial \phi g}{\partial x} v_{x} g t^{3}
\end{aligned}
$$

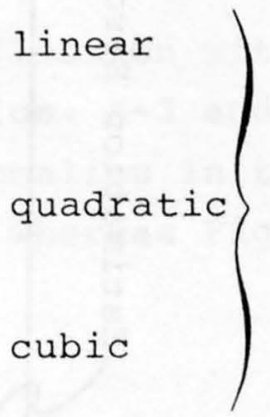

where

$$
\begin{aligned}
\psi_{X, y, z} & =\text { platform tilt angle errors } \\
v_{x} & =\text { airplane velocity } 175 \mathrm{ft} / \mathrm{sec} \\
b_{x, y} & =\text { accelerometer biases } \\
\frac{\partial \delta g}{\partial x} & =\text { change in gravity gradient with respect to } \\
\phi_{g} & \text { distance } \\
\varepsilon_{y} & =\text { gravimetric deflection drift rate } \\
\frac{\partial \phi g}{\partial x} & =\text { change in gravimetric deflection with respect to }
\end{aligned}
$$


In addition to the above approximate errors, there are errors due to the aircraft turn accelerations (A) multiplying both the gyro mass unbalance $(\stackrel{*}{U})$ and accelerometer scale factor errors $\left(\stackrel{*}{K}_{a}\right)$ in Eqs. $(E-2)$ and $(E-3)$. Every turn increases the error through this multiplication.

E.5 Simulation of IMU Errors in Initial 20-mile Pass Along Stream Valley

The computer numerically integrates the error differential equations of Figure E-2, including all of the effects mentioned above. Figure 4.1-10 of section 4.1.6 is a computer plot of the error growth in the IMU from the first to the third retroreflector during the initial 20-mile pass along the length of the stream valley. At $t=0$ the aircraft enters the river valley.

During this initial 20-mile pass, in actual operation, the walked-in retroreflectors will be surveyed from the air to determine their positions. In the simulation, these measurements are assumed to be in error $0.2 \mathrm{ft}$. in the vertical direction and $1.0 \mathrm{ft}$, in the horizontal.

Figure 4.1-10 shows all of the errors, except at the turns, from $t=120$ to $t=580$ seconds. Channel $x$ errors form the largest total because there the errors add. Channels $y$ and $\mathrm{z}$ form smaller totals because some of these errors cancel. Figure E-4 plots interaction with the second surveyed retroreflector and adds the effect of filtering and smoothing. To generate the data of Fig. E-4 the computer on board the aircraft estimates, while over the first retroreflector, the error sources (marked with $\rightarrow$ s in Fig. E-2), which are mostly coefficients, by comparing the position of the IMU to that measured by the tracker.

Using these new improved estimated error coefficients the error growth in the real inertial guidance system would 


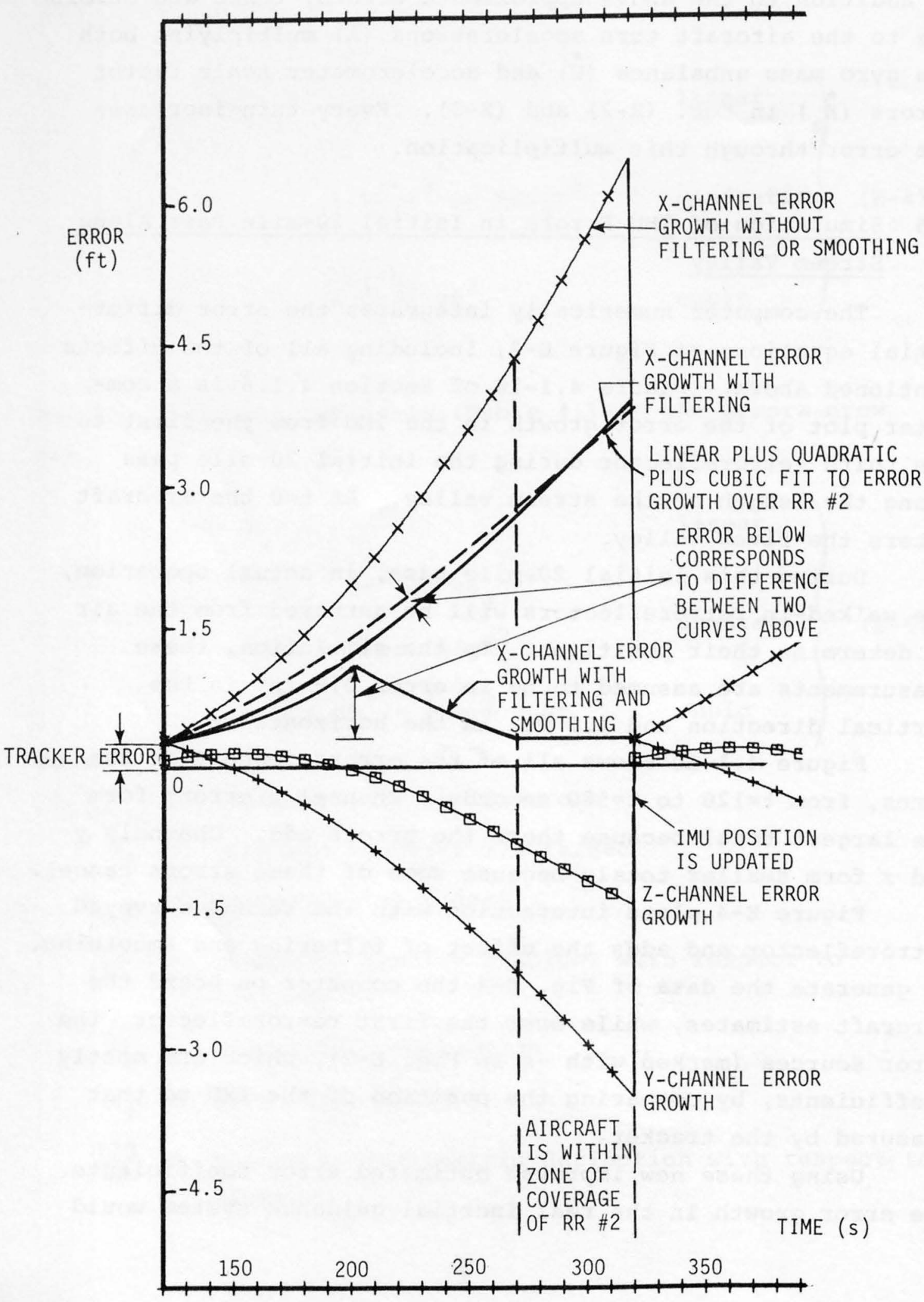

Fig. E-4. Longitudinal pass error growth with filtering and smoothing 
grow more slowly as shown by the solid line, second from top in Fig. E-4. Either the onboard computer or the post-flight computer would fit this filtered error growth to Eq. (E-5) with the result shown by the dashed line, third from top in Fig. E-4. (This equation is discussed in section 2.4 .2 and Appendices A.14 and C.5.)

Subtracting the fitted curve from the real curve, the difference is an estimate of the inertial system $x$ error (small at the ends, large at the middle). The $y$ and $z$ errors are calculated in similar fashion.

Figure E-4 plots this error as the filtered and smoothed curve. The scale in which this is plotted is exaggerated from the scale used in measuring the difference between the second and third curves described above, The maximum filtered and smoothed error in either horizontal channel (specifically, the $\mathrm{x}$-channel) is about $\mathrm{l} \mathrm{ft}$. The maximum filtered and smoothed error in the vertical channel is approximately $0.2 \mathrm{ft}$, but this is not shown in Fig. E-4.

\section{E.6 Determining Initial Conditions}

In the process described above the position error growth is corrected over the retroreflector. At the end of this period the $\mathrm{x}, \mathrm{y}$ and $\mathrm{z}$ errors drop down (are reset) to the accuracy of the laser tracker, calculated as follows. The slant range error of the tracker is assumed to be $0.1 \mathrm{ft}$. Pointing angle errors (pitch and roll) are assumed to be 20 secs. The resultant $x$ and $y$ errors, shown at the time of IMU update in Fig. E-4, were computed from the range of $3000 \mathrm{ft}$. and the angular error of $20 \mathrm{sec} .=10^{-4}$ radians, to be $0.3 \mathrm{ft}$. The $z$ error is the $0.1 \mathrm{ft}$. slant range error assumed above.

Assuming that the range is known with no error, the computed angular errors of the stable member of the IMU, 
when multiplied by the range of $3000 \mathrm{ft}$, result in additional errors of $0.21,0.15$ and 0.04 feet, respectively. These were not added to the above figures because those figures were already conservative.

The initial velocity error for $\mathrm{Eq} .(\mathrm{E}-3)$ was computed prior to simulation as follows. By subtracting range measurements and dividing by the time interval between, the velocity can be determined. The error in this velocity measurement averaged over the time the tracker is locked to the retroreflector $(50 \mathrm{sec}$.) is $.014 \mathrm{ft} / \mathrm{sec}$. in the $x$ and $y$ directions and $.0047 \mathrm{ft} / \mathrm{sec}$. in the $\mathrm{z}$ direction. These values are used as initial values in the error growth equation $(E-3)$ after updating.

To determine the tilt angle error over a surveyed retroreflector, Eq. (E-4) was fitted to the error growth of Eq. (E-3). From the time coefficients of Eq. (E-4) an estimate was made of the IMU angular errors by the simulation computer. (The accuracy of these estimates is limited by the laser tracker and gravity anomaly changes along the aircraft flight path.) The estimates of time coefficients were used to correct for the angular errors from Eq. (E-2) to an accuracy of

$$
\begin{aligned}
& \psi_{\mathrm{x}}=1.2 \times 10^{-5} \text { radians } \\
& \psi_{\mathrm{y}}=-0.7 \times 10^{5} \text { radians } \\
& \psi_{\mathrm{z}}=1.3 \times 10^{-4} \text { radians }
\end{aligned}
$$

These are initial conditions for Eq. (E-3) for error growth simulation. 


\section{E.7 Simulation of IMU Errors in A Valley Profile and Two Turns}

Figure 4.1-11 shows the position errors both for a valley profile (No. 10), that lies between two retroreflectors, and for two turns. Figure 4.11-9 shows the location of this profile in the simulated river valley. This is a worst case profile because there are no tracker data,

In Fig, 4.1-11 the greatest errors in $x$ and $y$ channels are shown to occur just before the $x$ and $y$ positions are updated from retroreflector data to the tracker error, calculated in Appendix E. The computer simulating both the valley and the inertial measuring system continues to generate data from the error equation, which can be employed to filter and smooth all of the data recorded for each channel, as was done for Fig. E-4. In an actual flight these data would be available only during update.

The vertical channel is updated at the center of profile No, 10, where this profile crosses the longitudinal pass.

It can be seen from Fig. 4.1-11 that the error buildup is about $8 \mathrm{ft}$. in the horizontal channel and about $4 \mathrm{ft}$. in the vertical channel. Filtering and smoothing as demonstrated in the longitudinal pass will reduce these values by a factor of at least 10 to fit within the budget of $6 \mathrm{in}$. in the vertical and $10 \mathrm{ft}$. in the horizontal. 



\section{APPENDIX F}

SIGNAL-TO-NOISE CONSIDERATIUNS FOR A PULSED LASER RANGEFINDER

The time interval to be measured is $2 \mathrm{~d} / \mathrm{c}$, where $\mathrm{d}$ is the distance (range) and $c$ is the speed of light. In the case of the tracker, for a maximum slant range of 2 kilometers, the corresponding time interval is approximately 13.33 usecs. For $0.5 \mathrm{ft}$. accuracy the resolution (least significant digit on time interval meter) would have to be somewhat better, such as 2 inches. A 2 -inch resolution corresponds to a $.33 \mathrm{nsec}$ time interval resolution or time delay error.

For a 0.33 nsec time delay error, the signal-to-noise ratio as a function of pulse width can be determined. The variance in the time delay error, $\sigma_{t}^{2}$, is equal to $\frac{1}{2 \mathrm{~B}_{\mathrm{S}}{ }^{2} \frac{\mathrm{S}}{\mathrm{N}}}$ where $B_{S}$ is the frequency spread and $\frac{S}{N}$ is the signal-to-noise ratio. For a rectangular pulse of width, $\mathrm{T}_{d}$, passed through an ideal filter extending in frequency from $-\frac{W}{2}$ to $+\frac{W}{2}$, $\mathrm{W}>\frac{1}{\mathrm{~T}_{\mathrm{d}}}, \mathrm{B}_{\mathrm{S}}{ }^{2} \approx \frac{2 \mathrm{~W}}{\mathrm{~T}_{\mathrm{d}}}$ and $\sigma_{t e}{ }^{2} \approx \frac{\mathrm{T}_{\mathrm{d}}}{4 \mathrm{~W} \frac{\mathrm{S}}{\mathrm{N}}}$, where $\mathrm{W}$ is the system bandwidth. Letting the rise time of the pulse $t_{r} \approx \frac{1}{\bar{W}}$, $\sigma_{t e}{ }^{2}=\frac{t_{r} T_{d}}{4 S / N} \cdot(\operatorname{Ref} \cdot 13)$ 
The following table shows the $\frac{\mathrm{S}}{\mathrm{N}}$ required to obtain a $\sigma_{t e}{ }^{2}=\left(.33 \times 10^{-9}\right)^{2}$ for various values of $t_{r}$ and $T_{d}$.

\begin{tabular}{rrr}
$\frac{\mathrm{S} / \mathrm{N}}{12.5}$ & $\frac{t_{r(n s)}}{\mathrm{T}_{\mathrm{d}(\mathrm{ns}}}$ & \multicolumn{1}{c}{5} \\
150 & 1 & 20 \\
2500 & 3 & 100 \\
10000 & 10 & 200
\end{tabular}


$\mathrm{P}_{\mathrm{b}}=\mathrm{H}_{\lambda}{ }^{\mathrm{A}} \mathrm{r} \mathrm{r}_{\mathrm{r}} \Delta \lambda \mathrm{T}_{0}\left[\frac{\rho_{\mathrm{b}} \mathrm{T}_{\mathrm{a}}}{\pi}+\frac{1}{4 \pi}\right]$ as given in section 4.2 .4

$\mathrm{H}_{\lambda}=.045 \mathrm{w} / \mathrm{m}^{2} \AA$ at $9000 \AA$ and $.14 \mathrm{w} / \mathrm{m}^{2} \AA$ at $6328 \AA$

$A_{r}=.0276 \mathrm{~m}^{2}$ for a $20 \mathrm{~cm}(8$-inch) receiving telescope (minus blockage) for the profiler and $5.04 \times 10^{-4} \mathrm{~m}^{2}$ for a one-inch receiver for the tracker.

Assuming a one-foot spot size from a 3000-foot altitude, $\Omega_{r} \simeq \frac{\pi D_{S}^{2}}{4 R^{2}} \approx \frac{\pi(1)^{2}}{4(3000)^{2}}=8.7 \times 10^{-8}$ steradians for the altimeter. The tracker will have a ten-foot spot size in the search mode, so that $\Omega=8.7 \times 10^{-6}$ steradians.

$\Delta \lambda=1 \AA$ for HeNe and $100 \AA$ for GaAs. The minimum optical bandwidth is limited by the temperature dependent wavelength shift of the laser diode.

$\mathrm{T}_{\mathrm{O}}=.5$

$\mathrm{T}_{\mathrm{a}}=1$ (worst case atmospheric transmission)

$\mathrm{P}_{\mathrm{b}}=.5$ (for this calculation assume average surface reflectivity of .5) 
At $6328 \AA$ for $20 \mathrm{~cm}$ telescope,

$$
\begin{aligned}
\mathrm{P}_{\mathrm{b}_{1}} & =.14(.0276)\left(8.7 \times 10^{-8}\right)(1)(.5)\left[\frac{.5}{\pi}+\frac{1}{4 \pi}\right] \\
& =4.02 \times 10^{-11} \text { watts }
\end{aligned}
$$

At $9000 \AA$ for tracker,

$$
\begin{aligned}
\mathrm{P}_{\mathrm{b}_{2}} & =.045\left(5.04 \times 10^{-4}\right)\left(8.7 \times 10^{-6}\right)(100)(.05)\left[\frac{.5}{\pi}+\frac{1}{4 \pi}\right] \\
& =2.34 \times 10^{-9} \text { watts }
\end{aligned}
$$

The corresponding background noise currents can be computed from $I_{B}=\frac{n e}{\hbar \nu} P_{b}, n=$ quantum efficiency, $e=$ electronic charge, $\mathrm{h}=$ Planck's constant and $\nu=$ optical frequency. With an S-1 photocathode at $9000 \AA, \quad n=.003$ With an S-20 photocathode at $6328 \AA, \eta=.04$

$$
\begin{aligned}
\nu & =3.33 \times 10^{14} \text { at } 9000 \AA \\
& =4.74 \times 10^{14} \text { at } 6328 \AA
\end{aligned}
$$

$$
\begin{aligned}
& \text { At } 9000 \AA \frac{n e}{h \nu}=2.18 \times 10^{-3} \\
& \text { At } 6328 \AA \frac{n e}{h \nu}=2.03 \times 10^{-2} \\
& I_{b_{1}}=8.16 \times 10^{-13} \text { amps (altimeter) } \\
& I_{b_{2}}=5.10 \times 10^{-12} \text { amps (tracker) }
\end{aligned}
$$


Note to Section $4,2,4$

\section{APPENDIX H}

SIGNAL-TO-NOISE RATIO CONSIDERATIONS AND RECEIVED POWER

Consider a sinusoidal signal plus wide bandwidth noise at the input of a narrow band filter. The output is

$$
g(t)=n(t)+A \cos (2 \pi f t+\theta)
$$

where $\mathrm{n}(t)$ is a narrow band gaussian random process, $f$ is the center frequency of the filter, $\theta$ is an arbitrary angle and $A$ is a constant.

The output of the filter may be rewritten as

$$
g(t)=R(t) \cos [(2 \pi f t+\phi(t))]
$$

where $R(t)$ and $\phi(t)$ are new envelope and phase functions with respect to $\mathrm{Eq}$. $(\mathrm{H}-\mathrm{l})$. The probability density of the phase is approximately

$$
P(\phi) \simeq \frac{A}{\sqrt{2} \pi \sigma} \exp -\left(\frac{A^{2}(\phi-\theta)^{2}}{2 \sigma^{2}}\right)
$$

which is a normal density function with variance $\sigma^{2} / A^{2}$ and mean $\theta$, Therefore,

$$
\overline{(\phi-\theta)^{2}}=\frac{\sigma^{2}}{A^{2}}=\frac{1}{2 \frac{S}{N}}
$$


Since the range $d=\frac{c \theta}{4 \pi f n_{g}}$, then

$$
\begin{aligned}
& \Delta \mathrm{d}_{\mathrm{rms}}=\frac{\mathrm{c}}{4 \pi f \mathrm{n}_{\mathrm{g}}} \quad \Delta \theta_{\mathrm{rms}} \\
& =\frac{c}{4 \pi f n_{g}}\left(\overline{\phi^{2}}\right)^{\frac{1}{2}} \\
& =\frac{c}{4 \pi f n_{g}} \frac{\sigma}{A} \\
& =\frac{\mathrm{c}}{4 \pi f \mathrm{n}_{g}} \frac{1}{\sqrt{2 \frac{\mathrm{S}}{N}}}
\end{aligned}
$$

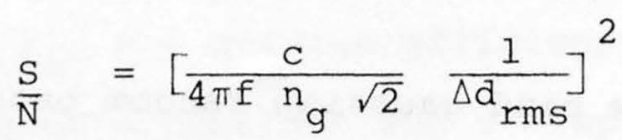

where $\frac{\mathrm{S}}{\mathrm{N}}$ is the signal-to-noise power ratio.

For the $50 \mathrm{MHz}$ modulation frequency (10-foot unambiguous range) and a $\Delta \mathrm{d}_{\mathrm{rms}}$ of 0.33 foot,

$$
\frac{\mathrm{S}}{\mathrm{N}}=11.25
$$

For the $5 \mathrm{MHz}$ modulation frequency (100-foot unambiguous range)

$$
\frac{\mathrm{S}}{\mathrm{N}}=1125
$$

The signal-to-noise current ratio (SNR) of a photomultiplier on the strong background flux region is given by $(4 \cdot 2-3)$.

$$
\text { SNR }=\frac{{ }^{M I_{r}}}{\left[\frac{\mathrm{kTBF}_{r}}{r}+2 e \mathrm{M}^{2} \mathrm{BF}_{\mathrm{m}}\left(I_{r}+I_{b}+I_{d}\right)\right]^{\frac{1}{2}}}
$$




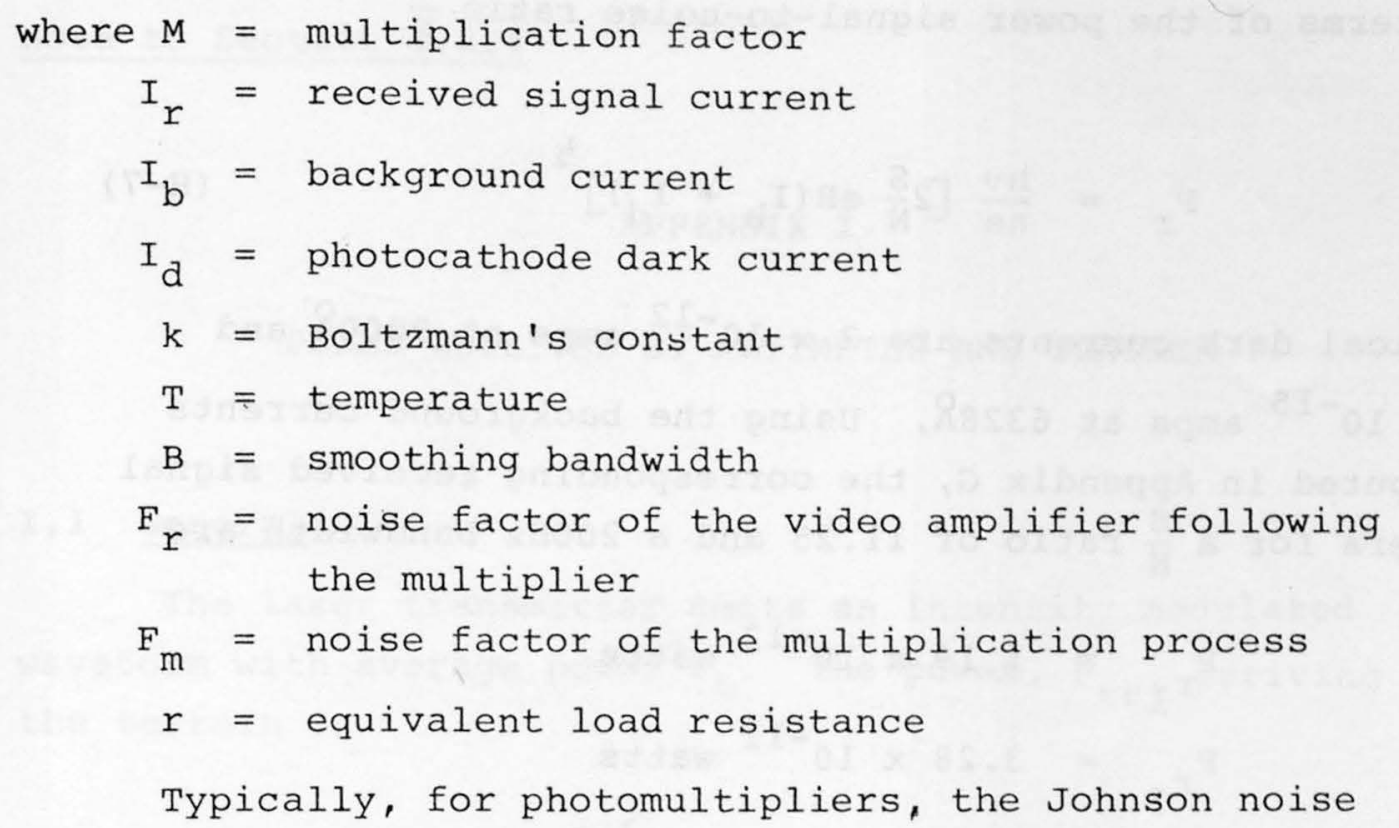
noise ratio can be expressed as

$$
\begin{aligned}
\text { SNR } & =\frac{I_{r}}{\left[2 e B\left(I_{r}+I_{b}+I_{d}\right)\right]^{\frac{1}{2}}} \\
\text { or } I_{r}{ }^{2} & =(S N R)^{2}\left[2 e B\left(I_{r}+I_{b}+I_{d}\right)\right]
\end{aligned}
$$

For moderate signal-to-noise ratios and small bandwidths,

$$
I_{r}>(\mathrm{SNR})^{2} 2 \mathrm{eB}
$$

Therefore $I_{r}{ }^{2}=2 e B\left(I_{b}+I_{d}\right)(S N R)^{2}$

$$
\begin{aligned}
& I_{r}=\operatorname{SNR}\left[2 e B\left(I_{b}+I_{d}\right)\right]^{\frac{1}{2}} \\
& P_{r}=\frac{h \nu}{n e} \operatorname{SNR}\left[2 e B\left(I_{b}+I_{d}\right)\right]^{\frac{1}{2}}
\end{aligned}
$$


In terms of the power signal-to-noise ratio $\frac{\mathrm{S}}{\mathrm{N}}$

$$
P_{r}=\frac{h \nu}{\eta e}\left[2 \frac{S}{N} e B\left(I_{b}+I_{d}\right)\right]^{\frac{1}{2}}
$$

Typical dark currents are $2 \times 10^{-12}$ amps at $9000 \AA$ and $1 \times 10^{-15} \mathrm{amps}$ at $6328 \AA$. Using the background currents computed in Appendix G, the corresponding received signal powers for a $\frac{\mathrm{S}}{\mathrm{N}}$ ratio of 11.25 and a $200 \mathrm{~Hz}$ bandwidth are

$$
\begin{aligned}
& \mathrm{P}_{r_{1}}=1.19 \times 10^{-12} \text { watts } \\
& \mathrm{P}_{r_{2}}=3.28 \times 10^{-11} \text { watts }
\end{aligned}
$$

For the higher signal-to-noise ratio corresponding to an unambiguous range of 100 feet, the above received powers would be multiplied by $\sqrt{10}$. 
Note to Section 4.2 .4

\section{APPENDIX I}

POWER RECEIVED BY ALTIMETER AND TRACKER

\section{1 General}

The laser transmitter emits an intensity modulated waveform with average power $\mathrm{P}_{t}$. The power, $\mathrm{P}_{t r}$, arriving on the terrain is

$$
P_{t r}=P_{t} \varepsilon^{-\sigma r}
$$

where $\sigma=$ atmospheric extinction coefficient

$$
r \quad=\text { range }
$$

\section{I.2 Laser Altimeter}

In the case of the laser altimeter or profiler, the terrain is assumed to be a plane Lambertian surface reflecting into a solid angle of $\pi$ steradians. The radiation reflected back into a cone of solid angle $\Omega$ steradians is

$$
\mathrm{P}_{\text {te }}=\rho \mathrm{P}_{\text {tr }} \frac{\Omega}{\pi}=\frac{\mathrm{P}_{\text {te }} \rho \varepsilon^{-\sigma \mathrm{r}} \Omega}{\pi}
$$

where $\rho$ is the reflectivity of the terrain.

The receiver with an aperture area $A_{r}$ at a range $r_{\text {, co- }}$ located with the transmitter, subtends a solid angle $\Omega=\frac{{ }^{A} r}{r^{2}}$ steradians. Thus, for the case where the target area is equal to or larger than the beamwidth and the receiver field 
of view is equal to or larger than the transmitter beamwidth, the received power

$$
P_{r}=\frac{{ }^{P_{t} \rho T_{r}} \varepsilon^{-2 \sigma r} A_{r}}{\pi r^{2}}
$$

and $(4.2-9)$

where

$$
\begin{aligned}
& \rho=\text { reflectivity of target area } \\
& T_{Y}=\text { transmission of the optics }
\end{aligned}
$$

\section{I.3 Laser Tracker}

In the case of the laser tracker, the retroreflector has a projected area $A_{r r}$ normal to the transmitted beam:

$$
A_{r r}=\frac{\pi d_{r r}^{2}}{4}
$$

where $\mathrm{d}_{r r}$ is the diameter of the retroreflector aperture (see Figs. 4.2-3 and I-I). For a laser beam of power $P_{t}$ and divergence solid angle of $\frac{\pi \alpha^{2}}{4}$ steradians, the irradiance incident upon the larger projected area is given by (14)

$$
\mathrm{E}_{\text {in }}=4 \mathrm{P}_{t} / \pi r^{2} \alpha^{2}
$$

The power $\mathrm{P}_{\text {rr }}$ incident on the retroreflector is given by

$$
P_{r r}=E_{i n} A_{r r}=\frac{4 P_{t}}{\pi r^{2} \alpha^{2}} \frac{\pi d_{r r}^{2}}{4}=\frac{P_{t} d_{r r}^{2}}{r^{2} \alpha^{2}}
$$

$\mathrm{P}_{\mathrm{rr}}$ is reflected back to the laser source within the angle of divergence $\frac{d_{r r}}{r}$. Additional divergence $\phi_{d}$ is introduced by the effects of diffraction and inaccuracy of the retroreflector. 


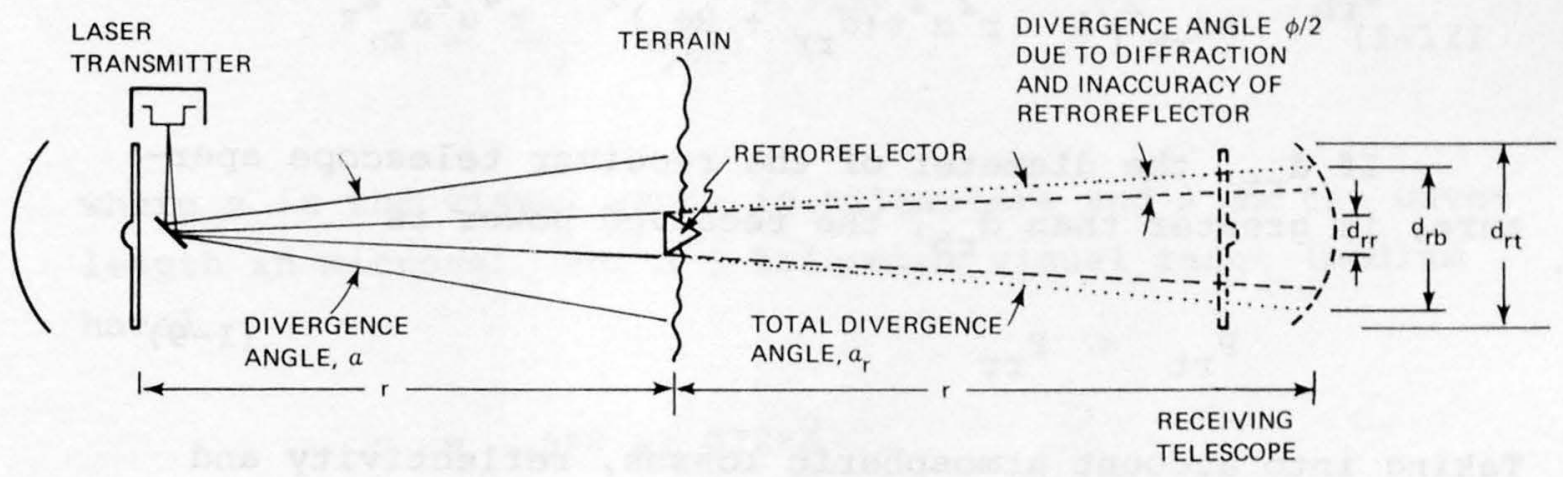

Fig. I-1. Diagram of optical tracker 
The diameter $d_{r b}$ of the return beam is then given by

$$
d_{r b}=2\left(d_{r r}+r \phi_{d}\right)=r \alpha_{r}
$$

where $\quad \alpha_{r}$ is the total divergence angle.

The irradiance of the return beam at the receiver telescope, $\mathrm{E}_{\mathrm{rb}}$, is then:

$$
E_{r b}=\frac{P_{r r}}{\pi d_{r b}^{2} / 4}=\frac{P_{t} d_{r r}{ }^{2}}{r^{2} \alpha^{2} \pi\left(d_{r r}+R \phi_{d}\right)^{2}}=\frac{P_{t} d_{r r}{ }^{2}}{r^{4} \alpha^{2} \alpha_{r}^{2} \pi}
$$

If $\mathrm{d}_{r t}$, the diameter of the receiver telescope aperture, is greater than $\mathrm{d}_{\mathrm{rb}}$ ' the received power is

$$
\mathrm{P}_{r t}=\mathrm{P}_{r r}
$$

Taking into account atmospheric losses, reflectivity and transmission losses

$$
P_{r t}=\rho T_{r} \frac{4}{\pi} \frac{{ }^{A}{ }^{A} r r}{r^{2} \alpha^{2}} \varepsilon^{-2 \sigma r}
$$

If $d_{r t}$ is less than $d_{r b}, P_{r t}$ is reduced by the ratio of the receiver aperture to the cross-section area of the beam, at the receiver, namely

$$
\frac{\frac{\pi d_{r t}^{2}}{4}}{\pi\left(\frac{r \alpha_{r}}{2}\right)}=\frac{d_{r t}^{2}}{r^{2} \alpha_{r}^{2}}
$$


Multiplying this ratio times $\mathrm{P}_{\text {rt }}$ yields

$$
P_{r t}^{\prime}=\frac{4 e^{T} r^{P} t^{A} r r^{d} r t}{\pi r^{4} \alpha^{2} \alpha_{r}{ }^{2}} \varepsilon^{-\sigma r}
$$

and $(4.2-10)$

To compute the atmospheric extinction coefficient, $\sigma$, the following expression is used:

$$
\sigma=\frac{3.91}{v}\left(\frac{\lambda}{.55}\right)^{-.585 v^{1 / 3}}
$$

where $\mathrm{v}$ is the visual range in kilometers and $\lambda$ is the wavelength in microns. For a 5-kilometer visual range (medium haze)

$$
\begin{aligned}
\sigma & =.680 \text { at } 6328 \AA \\
& =.478 \text { at } 9000 \AA
\end{aligned}
$$

Applying a correction factor of .69 for a l-kilometer altitude

$$
\begin{aligned}
\sigma & =.476 \text { at } 6328 \AA \\
& =.335 \text { at } 9000 \AA
\end{aligned}
$$





\section{APPENDIX J}

TRANSMITTER POWER DETERMINATION

\section{J.1 Laser Altimeter}

For the laser altimeter or profiler, the transmitted power can be expressed by rearranging Eq. (I-3):

$$
P_{t}=\frac{{ } r^{\pi}}{\rho T_{r}{ }^{A}} r^{2} \varepsilon^{2 \sigma r}
$$

The reflectivity, $\rho$, will be assumed to be a worst case of 0.1 . At the helium-neon wavelength of $6328 \AA$, according to Appendix I,

$$
\sigma=0.476
$$

The received power, according to Appendix $\mathrm{H}$, is

$$
P_{r}=1.19 \times 10^{-12} \text { watts }
$$

Taking into account the modulation process, transmitter and receiver optical losses,

$$
T_{r} \approx 0.16
$$


For the 8-inch diameter aperture, the area is

$$
A_{r}=\pi\left(4 \times 2.54 \times 10^{-2}\right)^{2}=3.2 \times 10^{-2} \mathrm{~m}^{2}
$$

Substituting the above values into $\mathrm{Eq}$. (I-3), for a range of $1 \mathrm{~km}$,

$$
\begin{aligned}
P_{t} & =\frac{1.19 \times 10^{-12} \pi}{0.1 \times 0.16 \times 3.2 \times 10^{-2}} \times 10^{6} \varepsilon 2(0.476) \\
& =18.9 \mathrm{~mW}
\end{aligned}
$$

\section{J.2 Laser Tracker}

For the laser tracker, the transmitted power can be expressed by rearranging Eq. (I-II)

$$
P_{t}=\frac{{ }_{r t}^{1} \pi r^{4} \alpha^{2} \alpha_{r}{ }^{2} \varepsilon^{2 \sigma r}}{{ }^{2} \rho^{T} r^{A} r{ }^{A}{ }^{2}{ }^{2}}
$$

Use the value of received power computed in Appendix $\mathrm{H}$, or $3.28 \times 10^{-11}$ watts, for a slant range of 2 kilometers. Let the diameter of the retroreflector be $0.5 \mathrm{ft}$. Then

$$
\begin{aligned}
A_{r r} & =0.196 \mathrm{ft}^{2} \times 0.1 \text { efficiency factor } \\
& \approx 0.02 \mathrm{ft}^{2} \approx 0.00186 \mathrm{~m}^{2}
\end{aligned}
$$

Let the divergence angle,

$$
\begin{aligned}
\alpha_{r}=10 \widehat{\mathrm{sec}}=2.64 \times 10^{-4} \text { radians } \\
\mathrm{P}_{t}=\frac{3.28 \times 10^{-11} \times \pi \times\left(2 \times 10^{3}\right)^{4}\left(3.33 \times 10^{-3}\right)^{2}\left(2.64 \times 10^{-4}\right)^{2} \varepsilon^{2(0.335)(2)}}{4(0.9)(0.5)\left(1.86 \times 10^{-3}\right)\left(6.45 \times 10^{-4}\right)} \\
=2.25 \mathrm{~mW}
\end{aligned}
$$


APPENDIX K

MODULATION FREQUENCY REQUIRED FOR DIFFERENT VALUES OF RANGE RESOLUTION

\begin{tabular}{|c|c|c|}
\hline 1 & 2 & 3 \\
\hline $\begin{array}{l}\text { Range } \\
\text { Resolution } \\
\text { (ft.) } \\
\\
0.01 \\
0.1 \\
1 . \\
10 .\end{array}$ & $\begin{array}{l}\text { Spacing of Unam- } \\
\text { biguous Readings } \\
=1000 \times \text { ( } 1 \text { ) ( } \mathrm{ft.})\end{array}$ & $\begin{array}{l}\text { Modulation } \\
\text { Frequency } \\
\text { Calculated } \\
\text { from (1) by } \\
\text { Eq. (4.2-3) } \\
50 \mathrm{MHz} \\
5 \mathrm{MHz} \\
500 \mathrm{KHz} \\
50 \mathrm{KHz}\end{array}$ \\
\hline
\end{tabular}


REFERENCES

1. Penny, M. F,, "Airborne Laser Profiling," Laser Applications Conference, Ian Clunies Ross Memorial Foundation, National Science Center, Parkville, Australia, oct. 1973.

2. Drohan, W. A., Inertial Navigation System Error Analysis, Draper Laboratory Publication, Cambridge, Ma., 1973.

3. Sliney, David H. and Benjamin C. Freasier, "Evaluation of Optical Radiation Hazards," Applied Optics, Jan. 1973.

4. FAA-RD-71-88, Airborne Flight Inspection Inertial

Locator Equipment; Development and Flight Evaluation; Final Report May 1971.

5. Golden, K. E., et al, "Laser Ranging System With $1 \mathrm{~cm}$. Resolution," Applied Optics, July 1973.

6. Froome, K. D., "Distance Measurement by Means of Modulated Light," Acta Frequenza, October 1972.

7. Stitch, M. L., "Laser Rangefinding," Chapter F7, Laser Handbook, edited by F. T. Arecchi and E. O. Schulz-DuBois, North Holland Publishing Co., 1972.

8. Pratt, W. K., Laser Communication Systems, Wiley, New York, 1969, Section 7.2.

9. Leondes, Guidance and Control of Aerospace Vehicles, McGraw-Hill, New York, N. Y., 1963, p. 113. 
References (Cont'd)

10. Etkin, Dynamics of Flight Stability and Control, John Wiley and Sons, New York, N. Y., 1966.

11. Broxmeyer, Charles, Inertial Navigation Systems, McGraw-Hill, N. Y., 1964, Chapter 2.

12. Terman, F. E., Electronic and Radio Engineering, McGraw-Hill Book Co., Inc., New York, N. Y., 1955, pp. 568-570.

13. Lindsay, W. C. and M. K. Simon, Telecommunication Engineering, Prentice-Hall, Inc., Englewood Cliffs, N. J., 1973, pp. 157-159.

14. RCA Electro-Optics Handbook, RCA Commercial Engineering Harrison, N. J., 1974, p. 10. 